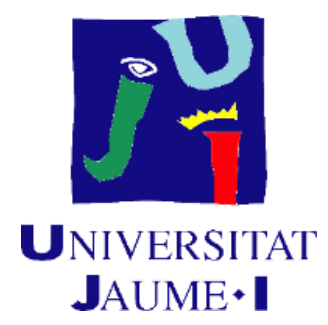

Programa de Doctorado en Tecnologías Industriales y Materiales

Escuela de Doctorado de la Universitat Jaume I

Advanced characterization of thermoelectric materials and devices by impedance spectroscopy

Memoria presentada por Braulio Beltrán Pitarch para optar al grado de doctor por la

Universitat Jaume I

Author:

Braulio Beltrán Pitarch

BRAULIO|

BELTRAN|

PITARCH

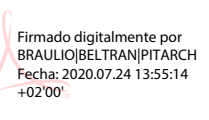

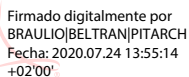

Directed by:

Dr. Jorge García Cañadas

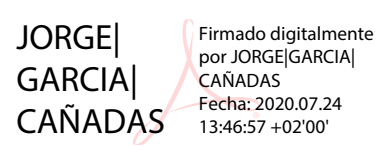

Castelló de la Plana, July 2020 


\section{Financial support}

To carry out the research in this thesis, financial support from the following institutions and projects has been received:

1. The Generalitat Valenciana and the European Social Fund co-financed the PhD grant (ACIF/2018/233) and the research stay grant (BEFPI/2019/030) of the PhD candidate under the ACIF and BEFPI programs, respectively.

2. The Department of Industrial Systems Engineering and Design of the Universitat Jaume I funded a collaborative scholarship (number 654) for 2 months.

3. The Spanish Agencia Estatal de Investigación funded part of the research with the grant of the PhD supervisor (RYC-2013-13970) under the Ramón y Cajal program.

4. The Universitat Jaume I funded the projects UJI-A2016-08 and UJI-B2019-50. 
Thesis by compendium of publications:

1. B. Beltrán-Pitarch, J. Prado-Gonjal, A. V. Powell, P. Ziolkowski, and J. García-Cañadas. Thermal conductivity, electrical resistivity, and dimensionless figure of merit (ZT) determination of thermoelectric materials by impedance spectroscopy up to $250{ }^{\circ} \mathrm{C}$. Journal of Applied Physics 124, 025105 (2018).

2. B. Beltrán-Pitarch, J. Prado-Gonjal, A. V. Powell, F. Martinez-Julian, and J. GarcíaCañadas. Complete Characterization of Thermoelectric Materials by Impedance Spectroscopy. Journal of Physical Chemistry C 123, 12608-12613 (2019).

3. B. Beltrán-Pitarch and J. García-Cañadas. Influence of convection at outer ceramic surfaces on the characterization of thermoelectric modules by impedance spectroscopy. Journal of Applied Physics 123, 084505 (2018).

4. B. Beltrán-Pitarch, F. Vidan and J. García-Cañadas. Characterization of thermal contacts between heat exchangers and a thermoelectric module by impedance spectroscopy. Applied Thermal Engineering 165, 114361 (2019).

5. B. Beltrán-Pitarch, J. Maassen, and J. García-Cañadas. Detailed assessment of thermoelectric modules by a comprehensive impedance spectroscopy equivalent circuit. (To be submitted).

"This thesis has been accepted by the co-authors of the publications listed above that have waved the right to present them as part of another $\mathrm{PhD}$ thesis" 


\section{Acknowledgments}

First, I would like to thank my supervisor Dr. Jorge García-Cañadas for his dedication and guidance since we started working together. His comments and explanations have been very useful for me, professionally and personally. I really think he could not have done a better job!

I am also grateful to all coauthors of my publications and, especially, Dr. Jesse Maassen for hosting me in his lab during my research stay at Dalhousie University. He is an amazing human being as well as supervisor.

Then, I would like to express my gratitude to all the colleagues currently working in this research group and the ones who have already left. It is nice to be part of a group where each of us is always happy to help the others. I would also like to thank Jaume, who usually joins us during lunch time, for his wisdom and willingness to share his knowledge. The technical support of the technicians Pepe and Raquel, the whole department, and the entire Universitat Jaume I, is also acknowledged.

It was a pleasure to perform a research stay at Dalhousie University and I would like to say thank you to all the fantastic people I met there. In this regard, I would like to highlight the hospitality of Karan and his family for hosting me in that awesome trip to Toronto. Halifax is a nice and quiet place and I felt like I could live there forever.

On the other hand, I would like to thank my family and lifelong friends for caring about me without clearly understand what all of this is about. I will never forget all of you waiting for me the day I came back from Canada. It was nice to see all of you again!

Dr. Anthony Powell, Dr. Jesús Prado-Gonjal, and Dr. Pawel Ziolkowski are acknowledged for providing skutterudite samples. European Thermodynamics is also acknowledged for providing thermoelectric devices.

Finally, I would like to acknowledge financial support from the Generalitat Valenciana and the European Social Fund under the ACIF program (ACIF/2018/233) and BEFPI program (BEFPI/2019/030), from the Department of Industrial Systems Engineering and Design of the Universitat Jaume I for the collaborative grant (number 654), from the Spanish Agencia Estatal 
de Investigación under the Ramón y Cajal program (RYC-2013-13970), and from the Universitat Jaume I under the projects UJI-A2016-08 and UJI-B2019-50. 


\section{List of abbreviations and symbols}

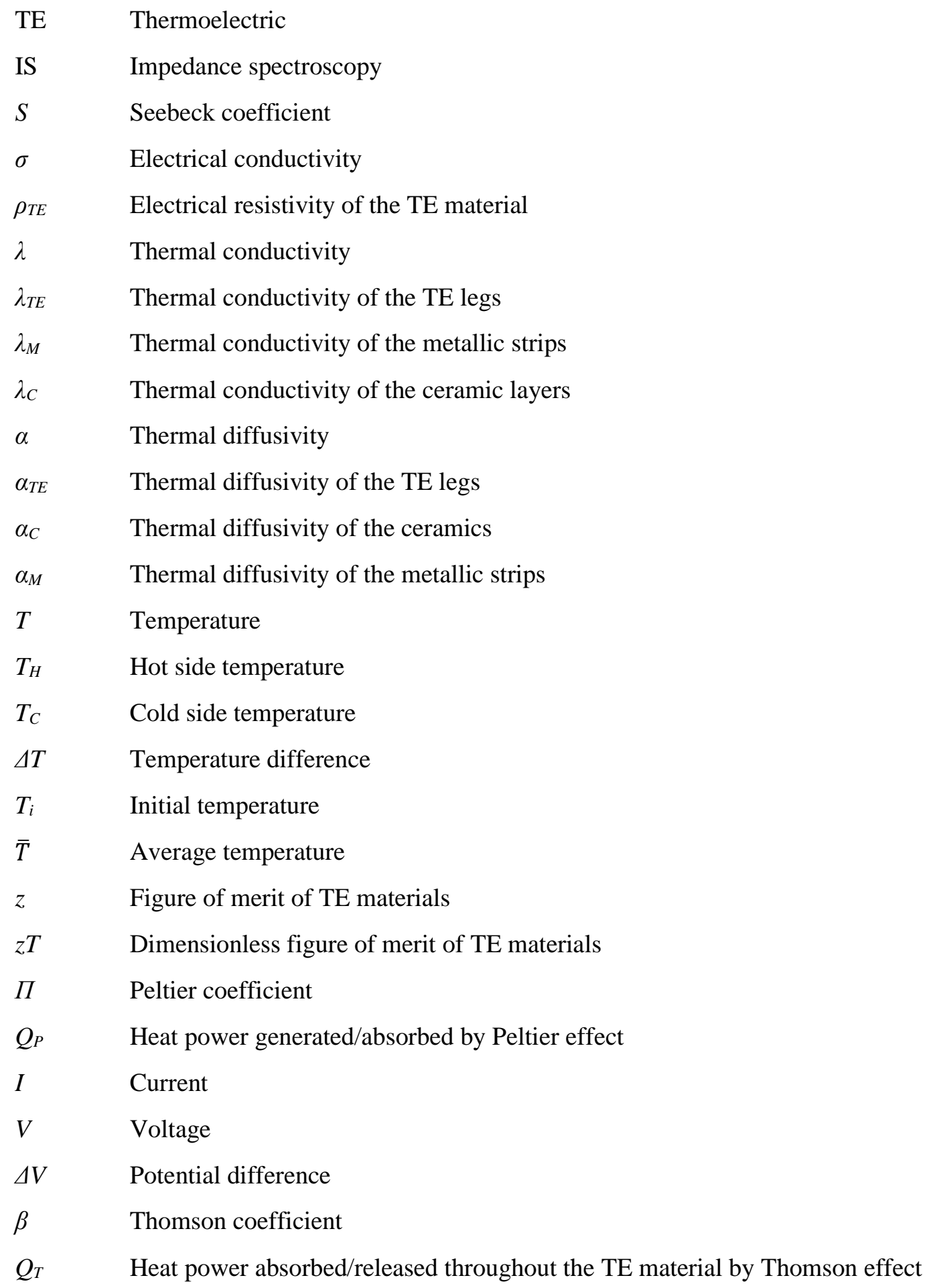




\begin{tabular}{|c|c|}
\hline$N$ & Number of couples of a TE device \\
\hline$S_{m}$ & Seebeck coefficient of TE modules \\
\hline$R_{\Omega}$ & Total ohmic resistance \\
\hline$R_{\text {load }}$ & Load resistance \\
\hline$\Delta V_{\text {load }}$ & Potential difference of the load \\
\hline$P_{\text {out }}$ & Power output of TE modules \\
\hline$P_{\operatorname{Max}}$ & Maximum power output \\
\hline$\eta_{m}$ & Efficiency of TE modules \\
\hline$Q_{\text {in }}$ & Heat power input \\
\hline$K_{m}$ & Total thermal conductance of TE modules \\
\hline$Z_{m}$ & Figure of merit of TE modules \\
\hline$Z_{m} T$ & Dimensionless figure of merit of TE modules \\
\hline COP & Coefficient of performance \\
\hline $\mathrm{COP}_{c}$ & Coefficient of performance for cooling \\
\hline $\mathrm{COP}_{h}$ & Coefficient of performance for heating \\
\hline$Q_{\text {out }, c}$ & Heat power extracted from the cold side \\
\hline$Q_{\text {out }, h}$ & Heat power generated at the hot side \\
\hline$P_{\text {in }}$ & Electrical power supplied to the TE module \\
\hline$f$ & Frequency \\
\hline$j$ & Imaginary number \\
\hline$\omega$ & Angular frequency \\
\hline$I_{a c}$ & Current amplitude of an ac signal \\
\hline$I_{d c}$ & Fixed (dc) current of an ac signal \\
\hline$V_{a c}$ & Voltage amplitude of an ac signal \\
\hline$V_{d c}$ & Fixed $(\mathrm{dc})$ voltage of an ac signal \\
\hline$Z$ & Impedance \\
\hline$|Z|$ & Module of the impedance \\
\hline$Z^{\prime}$ & Real impedance \\
\hline$Z^{\prime \prime}$ & Imaginary impedance \\
\hline
\end{tabular}




\begin{tabular}{|c|c|}
\hline$\varphi$ & Phase \\
\hline$\theta$ & Laplace transform of the temperature with respect to the initial temperature \\
\hline$\theta_{3}$ & Laplace transform of the excess temperature at outer ceramic surfaces \\
\hline$i_{0}$ & Laplace transform of the current at $x=0$ \\
\hline$\phi_{x}$ & Heat flow in the frequency domain at position $x$ \\
\hline$y_{T E}$ & Admittance towards the TE elements \\
\hline$y_{e}$ & Admittance towards the metallic strips \\
\hline$y_{0}$ & Total thermal admittance \\
\hline$R$ & Resistor \\
\hline$C$ & Capacitor \\
\hline$Z_{W C T}$ & Constant-temperature Warburg \\
\hline$Z_{W a}$ & Adiabatic Warburg \\
\hline$R_{T E}$ & TE resistance \\
\hline$R_{M}$ & TE resistance induced by the metallic strips \\
\hline$R_{C}$ & TE resistance induced by the ceramics \\
\hline$\omega_{c r}$ & Characteristic angular frequency \\
\hline$\omega_{T E}$ & Characteristic angular frequency of the TE legs \\
\hline$\omega_{M}$ & Characteristic angular frequency of the metallic strips \\
\hline$\omega_{C}$ & Characteristic angular frequency of the ceramics \\
\hline$L$ & Length of the TE elements \\
\hline$L_{M}$ & Length of the metallic strips \\
\hline$L_{C}$ & Length of the ceramic contacts \\
\hline$A$ & Area \\
\hline$\eta$ & TE module filling factor, ratio area legs/ceramics \\
\hline$\eta_{M}$ & Ratio between area of all TE legs and all metallic strips \\
\hline$v$ & Number of legs removed to attach the leads of the TE device \\
\hline$C_{p}$ & Specific heat \\
\hline$C_{p, M}$ & Specific heat of the metallic strips \\
\hline$C_{p, C}$ & Specific heat of the ceramic material \\
\hline
\end{tabular}




\begin{tabular}{|c|c|}
\hline$d$ & Mass density \\
\hline$d_{M}$ & Mass density of the metallic strips \\
\hline$d_{C}$ & Mass density of the ceramic material \\
\hline$e_{M}$ & Thermal effusivity of the metallic strips \\
\hline$e_{C}$ & Thermal effusivity of the ceramic material \\
\hline$\sigma_{B}$ & Stefan-Boltzmann constant \\
\hline$\varepsilon_{T E}$ & Emissivity of TE material \\
\hline$\varepsilon_{M}$ & Emissivity of metallic strips' material \\
\hline$\varepsilon_{C}$ & Emissivity of ceramic material \\
\hline$h$ & Convection heat transfer coefficient \\
\hline$h_{i c}$ & Internal convection heat transfer coefficient \\
\hline$h_{e c}$ & External convection heat transfer coefficient \\
\hline$h_{0}$ & Convection/radiation heat transfer coefficient around TE legs \\
\hline$h_{1}$ & Convection/radiation heat transfer coefficient around metallic strips \\
\hline$h_{2}$ & Convection/radiation heat transfer coefficient at inner ceramic surfaces \\
\hline$h_{3}$ & Convection/radiation heat transfer coefficient at outer ceramic surfaces \\
\hline$R_{\text {conv }}$ & Convection resistance \\
\hline$C_{c o n v}$ & Convection capacitance \\
\hline$r_{T C}$ & Thermal contact resistivity between TE module and heat exchangers \\
\hline$R_{T C}$ & Thermal contact electrical resistance \\
\hline$C_{T C}$ & Thermal contact capacitance \\
\hline$r_{T C l}$ & Thermal contact resistivity between TE legs and metallic strips \\
\hline$r_{T C 2}$ & Thermal contact resistivity between metallic strips and ceramics \\
\hline$R_{T C l}$ & Resistor induced by $r_{T C l}$ \\
\hline$R_{T C 2}$ & Resistor induced by $r_{T C 2}$ \\
\hline$C_{T C 2}$ & Capacitor induced by $r_{T C 2}$ \\
\hline$r_{M}$ & Equivalent radius of the metallic strips \\
\hline$r_{C}$ & Equivalent radius of the ceramic layers \\
\hline$J_{0}$ & First kind Bessel function of order zero \\
\hline
\end{tabular}




$\begin{array}{ll}J_{l} & \text { First kind Bessel function of order one } \\ \delta_{n} & \text { Each of the zeros of } J_{l} \\ z_{s / c} & \text { Spreading-constriction impedance } \\ r_{s / c} & \text { Spreading-constriction resistivity } \\ Z_{S / C} & \text { Impedance element induced by } z_{s} / c \\ Z_{i, M} & \text { Impedance element (of impedance function } i \text { ) induced by the metallic strips } \\ R_{d c} & \text { Resistance when } \omega \rightarrow 0 \text { (dc resistance) } \\ f_{p} & \text { Each of the properties used to calculate the error } \\ x_{i} & \text { Each of the parameters required to determine } f_{p} \\ u & \text { Error of properties } \\ u_{c} & \text { Total combined random error } \\ u_{s} & \text { Systematic error } \\ u_{T} & \text { Total error }\end{array}$




\section{Abstract}

It is estimated that around 60\% of the global energy used is lost as waste heat. Thermoelectric (TE) devices can directly convert heat into electricity (energy generation mode) or use electricity to create a temperature difference (cooling/heating mode). For this reason, they can be a suitable energy harvesting technology and contribute to the current energy crisis. However, they are not widely spread currently due to their low efficiency. The development of new, more efficient materials is typically based on the optimization of the dimensionless figure of merit $\left(z T=S^{2} \sigma T / \lambda\right)$, which is determined by three material properties: the Seebeck coefficient $(S)$, the electrical conductivity $(\sigma)$ and the thermal conductivity $(\lambda)$, and also the temperature $(T)$. Hence, the determination of these properties as a function of temperature is a necessary step in the development of any new material.

Regrettably, the characterization of all these parameters is quite lengthy and tedious, typically requiring the use of at least two different apparatuses. In addition, there is not a standard equipment established and homemade apparatuses are frequently employed, which makes difficult inter-laboratory correlation. Moreover, a high uncertainty is typically associated with $z T$, since it adds the errors of the three properties that define it.

Impedance spectroscopy (IS) is successfully used as a standard characterization technique in many different research fields (batteries, capacitors, coatings, photovoltaics, etc.) and could be also very useful for TEs. However, only a few studies showed the potential of this technique to characterize TE materials and devices at the beginning of this thesis. For this reason, the main objective of this work is to advance the application of IS in the TE field in order to potentially establish it as a standard method in thermoelectricity.

Our results have shown the possibility to determine $\sigma, \lambda$ and $z T$ in TE materials with good accuracy at temperatures up to $250^{\circ} \mathrm{C}$ using IS, even for a material with modest TE properties. Moreover, we developed a new method capable of determining all these properties and the Seebeck coefficient (complete characterization) in a single IS measurement. We also extended the initial ideal impedance models (equivalent circuits) for the characterization of TE modules to include the convection effect at the outer ceramic surfaces for suspended TE devices, and the effect of the thermal contact resistance between the TE device and ideal heat sinks for sandwiched 
configurations. Finally, we developed a more comprehensive equivalent circuit, which includes all the key phenomena that could take place in a TE device. This model included the metallic strips that connect the TE legs in a TE device, the thermal contact resistances inside the TE module (TE legs/metallic strips and metallic strips/ceramic layers), and the spreading-constriction effects.

All these developments establish the impedance method as a powerful technique in thermoelectricity, allowing the complete characterization of TE materials from a single measurement, opening up the possibility of using this technique as a tool to quantify and monitor relevant thermal parameters, such as the convection heat transfer coefficient $(h)$ and thermal contact resistances. Furthermore, our results also open the possibility of using IS as a quality control tool to detect and monitor in great detail issues in TE devices. 


\section{Abstract (Spanish version)}

Se estima que alrededor del $60 \%$ de la energía global utilizada se pierde en forma de calor residual. Los dispositivos termoeléctricos (TEs) pueden convertir directamente el calor en electricidad (modo de generación de energía) o usar electricidad para crear una diferencia de temperatura (modo de refrigeración/calefacción). Por esta razón, pueden ser una tecnología adecuada de recuperación de energía y contribuir a la crisis energética actual. Sin embargo, actualmente no están muy extendidos debido a su baja eficiencia. El desarrollo de nuevos materiales más eficientes se basa típicamente en la optimización de la figura de mérito adimensional $\left(z T=S^{2} \sigma T / \lambda\right)$, que está determinada por tres propiedades del material: el coeficiente Seebeck $(S)$, la conductividad eléctrica $(\sigma)$ y la conductividad térmica $(\lambda)$, y también la temperatura $(T)$. Por lo tanto, la determinación de estas propiedades en función de la temperatura es un paso necesario en el desarrollo de cualquier material nuevo.

Lamentablemente, la caracterización de todos estos parámetros requiere tiempo, es tediosa y normalmente emplea al menos dos equipos diferentes. Tampoco existe un procedimiento estándar de medida y con frecuencia se utilizan montajes caseros, lo que dificulta la correlación de medidas entre laboratorios. Además, la $z T$ lleva asociada una alta incertidumbre ya que agrega los errores de las tres propiedades que la definen.

La espectroscopía de impedancia (IS) se utiliza con éxito como técnica de caracterización estándar en muchos campos de investigación diferentes (baterías, condensadores, recubrimientos, celdas fotovoltaicas, etc.) y también se podría emplear en termoelectricidad. Sin embargo, solo unos pocos estudios mostraron el potencial de esta técnica para caracterizar los materiales y dispositivos TEs al comienzo de esta tesis. Por esta razón, el objetivo principal de este trabajo es avanzar en la aplicación de la IS en el campo de la termoelectricidad para establecerla potencialmente como un método estándar en este campo.

Nuestros resultados han mostrado la posibilidad de determinar $\sigma, \lambda$ y $z T$ en materiales TEs con buena precisión a temperaturas de hasta $250{ }^{\circ} \mathrm{C}$ utilizando la espectroscopía de impedancia, incluso para un material con modestas propiedades TE. Además, desarrollamos un nuevo método capaz de determinar todas estas propiedades y el coeficiente Seebeck (caracterización completa) en una sola medida de IS. También ampliamos los modelos de impedancia teóricos ideales 
(circuitos equivalentes) de caracterización de módulos TEs para incluir el efecto de convección en las superficies cerámicas exteriores en dispositivos TEs suspendidos, y el efecto de la resistencia térmica de contacto entre el dispositivo TE e intercambiadores de calor ideales para dispositivos ensamblados. Finalmente, desarrollamos un circuito equivalente más completo, que incluye todos los fenómenos clave que podrían tener lugar en un dispositivo TE. Este modelo incluye las tiras metálicas que conectan los elementos TEs en un dispositivo TE, las resistencias térmicas de contacto dentro del módulo TE (elementos TEs/tiras metálicas y tiras metálicas/contactos cerámicos) y los efectos de expansión y constricción del calor al pasar de un material a otro con área diferente.

Todos estos desarrollos establecen el método de impedancia como una técnica potente en termoelectricidad, permitiendo la caracterización completa de materiales TEs a partir de una sola medida y la posibilidad de usar esta técnica como una herramienta para cuantificar parámetros térmicos relevantes, como el coeficiente de transmisión de calor por convección $(h)$ y las resistencias térmicas de contacto. Además, nuestros resultados también muestran la posibilidad de utilizar la IS como una herramienta de control de calidad para detectar y monitorizar con gran detalle problemas en dispositivos TEs. 


\section{Abstract (Valencian version)}

S'estima que al voltant del $60 \%$ de l'energia global utilitzada es perd com calor residual. Els dispositius termoelèctrics (TEs) poden convertir directament calor en electricitat (en mode generació d'energia) o usar electricitat per crear una diferència de temperatura (en mode refrigeració/calefacció). Per aquesta raó, poden ser una tecnologia adequada de recuperació d'energia i contribuir positivament a la crisi energètica actual. No obstant això, actualment no estan molt estesos a causa de la seua baixa eficiència. El desenvolupament de nous materials més eficients es basa típicament en l'optimització de la figura de mèrit adimensional $\left(z T=S^{2} \sigma T / \lambda\right)$, que està determinada per tres propietats del material: el coeficient Seebeck $(S)$, la conductivitat elèctrica $(\sigma)$ i la conductivitat tèrmica $(\lambda)$, i també la temperatura $(T)$. Per tant, la determinació d'aquestes propietats en funció de la temperatura és un pas necessari en el desenvolupament de qualsevol material nou.

Lamentablement, la caracterització de tots aquests paràmetres requereix temps, és tediosa $\mathrm{i}$ normalment necessita, almenys, dos equips diferents. Tampoc existeix un procediment estàndard de mesura i amb freqüència s'utilitzen muntatges casolans, la qual cosa dificulta la correlació de mesures entre laboratoris. A més a més, la $z T$ porta associada una alta incertesa ja que inclou els errors de les tres propietats que la defineixen.

La espectroscòpia d'impedància (IS) s'utilitza amb èxit com a tècnica de caracterització estàndard en molts camps d'investigació diferents (bateries, condensadors, recobriments, cel·les fotovoltaiques, etc.) i també es podria emprar en termoelectricitat. No obstant això, només uns pocs estudis van mostrar el potencial d'aquesta tècnica per a caracteritzar els materials i dispositius TEs al començament d'aquesta tesi. Per aquesta raó, l'objectiu principal d'aquest treball és avançar en l'aplicació de la IS en el camp de la termoelectricitat per a establir-la potencialment com un mètode estàndard en aquest camp.

Els nostres resultats han mostrat la possibilitat de determinar $\sigma, \lambda \mathrm{i} z T$ en materials TEs amb bona precisió a temperatures de fins a $250{ }^{\circ} \mathrm{C}$ utilitzant la IS, fins i tot per a un material amb propietats TE modestes. A més a més, hem desenvolupat un nou mètode capaç de determinar totes aquestes propietats i el coeficient Seebeck (caracterització completa) amb una sola mesura de IS. També ampliem els models d'impedància teòrics ideals (circuits equivalents) de 
caracterització de mòduls TEs per incloure l'efecte de convecció a les superfícies ceràmiques exteriors de dispositius TEs suspesos, i l'efecte de la resistència tèrmica de contacte entre el dispositiu TE i intercanviadors de calor ideals per a dispositius ja acoblats. Finalment, hem desenvolupat un circuit equivalent més complet, que inclou tots els fenòmens clau que podrien tindre lloc en un dispositiu TE. Aquest model inclou les tires metàl-liques que connecten els elements TEs en un dispositiu TE, les resistències tèrmiques de contacte dins del mòdul TE (elements TEs/tires metàl-liques i tires metàl-liques/contactes ceràmics) i els efectes d'expansió i constricció de la calor en passar d'un material a un altre amb àrea diferent.

Tots aquests desenvolupaments estableixen el mètode d'impedància com una tècnica potent en termoelectricitat, permetent la caracterització completa de materials TEs a partir d'una sola mesura i l'ús aquesta tècnica com una eina per a quantificar paràmetres tèrmics rellevants, com el coeficient de transmissió de calor per convecció $(h)$ i les resistències tèrmiques de contacte. A més a més, els nostres resultats també mostren la possibilitat d'utilitzar la IS com una eina de control de qualitat per a detectar i monitoritzar amb gran detall problemes en dispositius TEs. 


\section{Index}

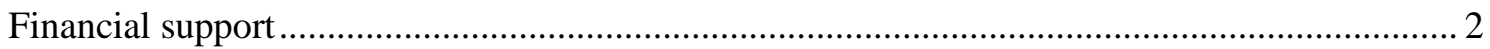

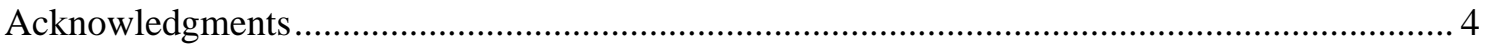

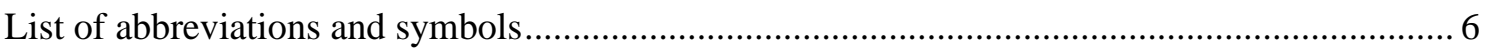

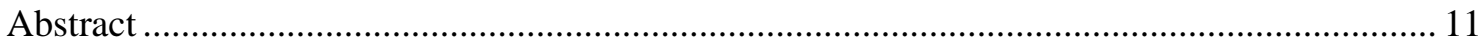

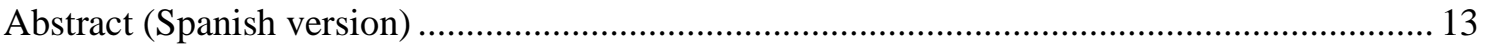

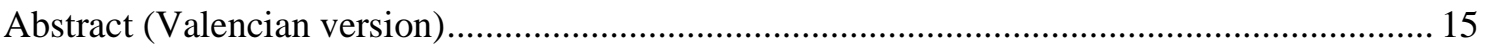

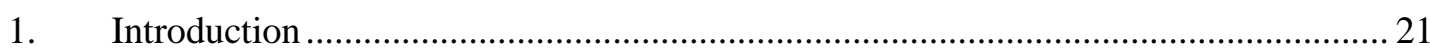

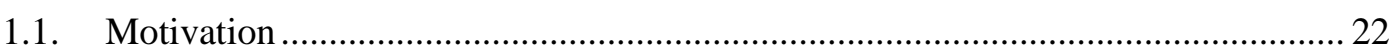

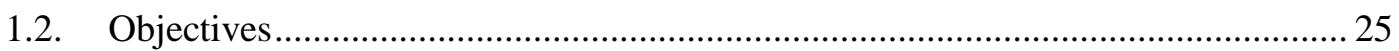

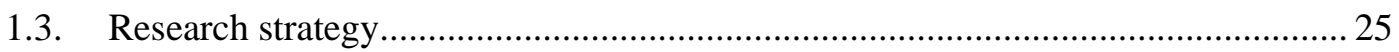

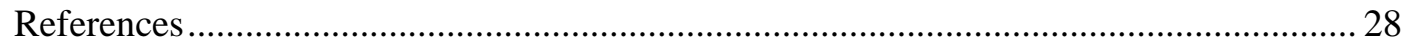

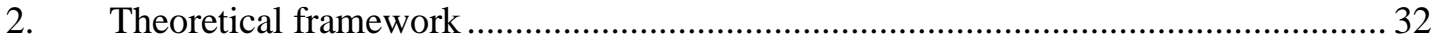

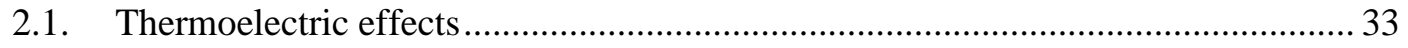

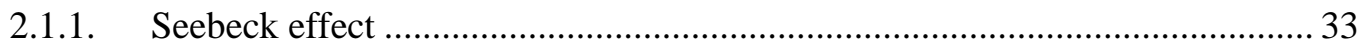

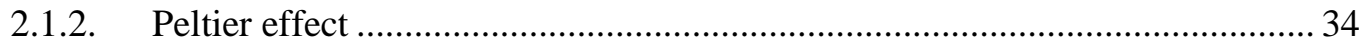

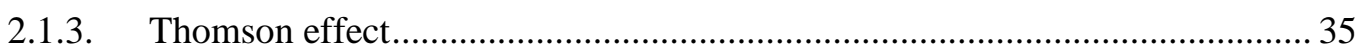

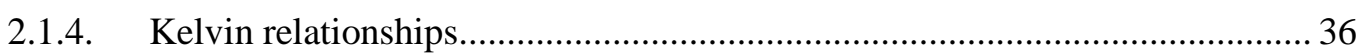

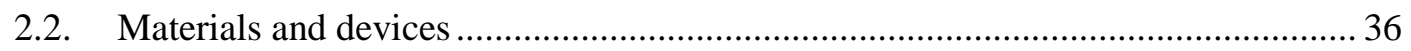

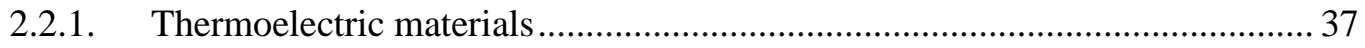

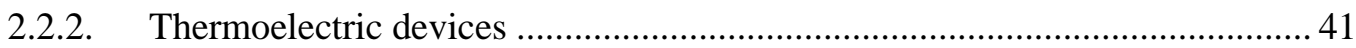

2.2.3. Power output of thermoelectric devices ....................................................... 42

2.2.4. Efficiency of thermoelectric devices .............................................................. 43

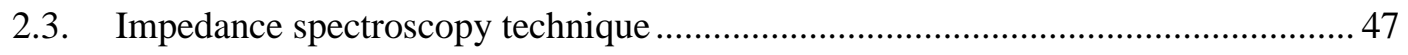

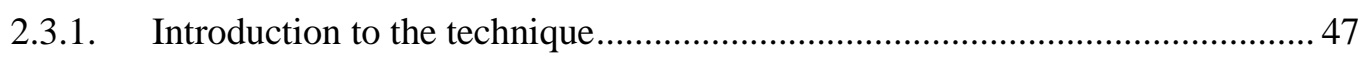

2.3.2. Impedance spectroscopy in thermoelectricity ............................................ 49

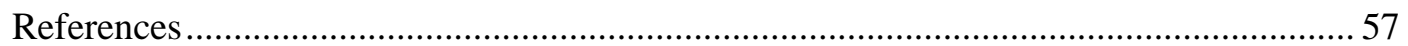

3. Thermal conductivity, electrical resistivity, and dimensionless figure of merit $(z T)$ determination of thermoelectric materials by impedance spectroscopy up to $250{ }^{\circ} \mathrm{C}$. 63

Abstract 65 


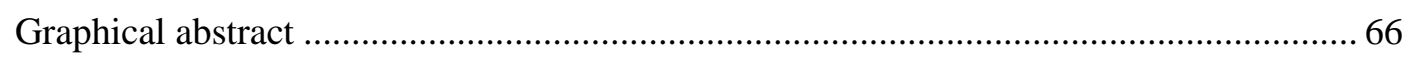

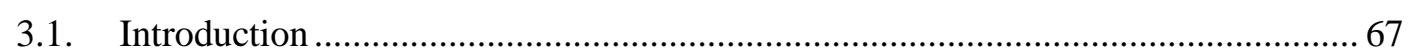

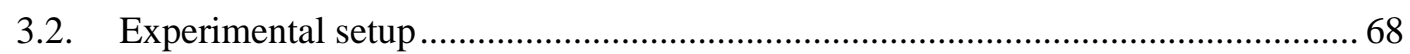

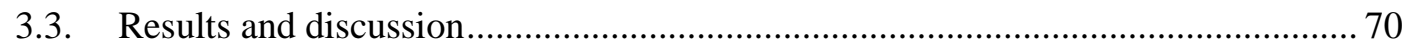

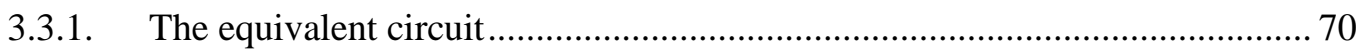

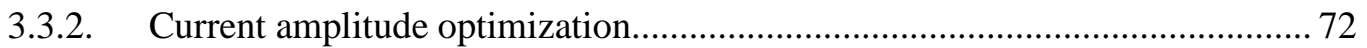

3.3.3. Characterization by the impedance method ..................................................... 75

3.3.4. Precision and accuracy evaluation ............................................................... 76

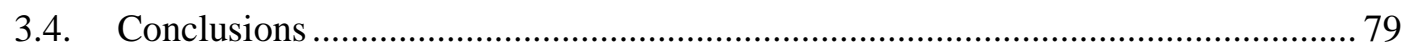

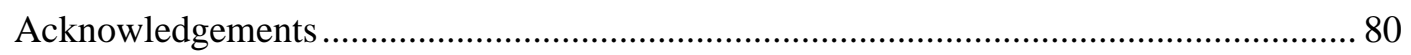

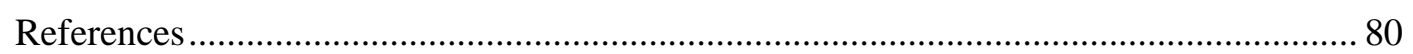

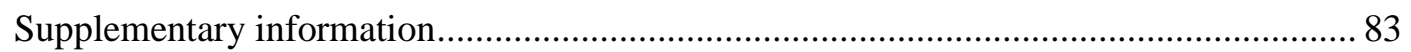

4. Complete characterization of thermoelectric materials by impedance spectroscopy .. 87

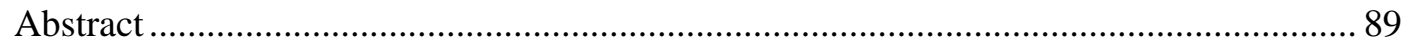

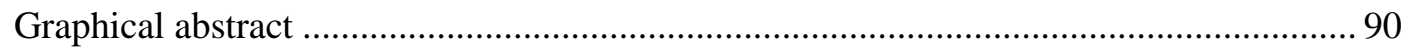

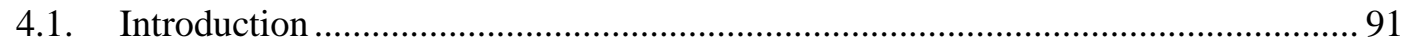

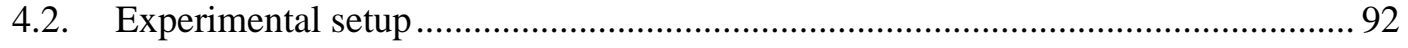

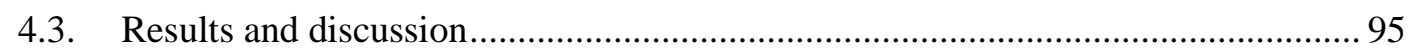

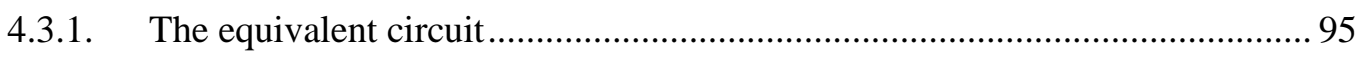

4.3.2. Characterization by the impedance method ................................................... 96

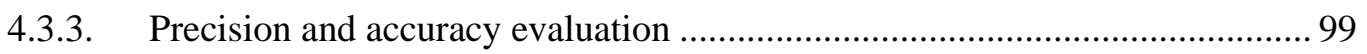

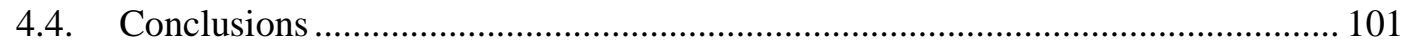

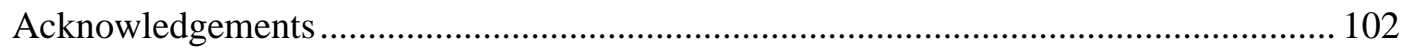

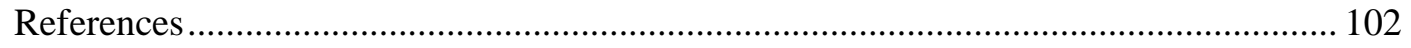

5. Influence of convection at outer ceramic surfaces on the characterization of thermoelectric modules by impedance spectroscopy …......................................................... 107

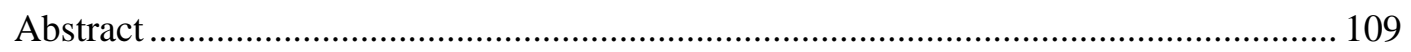

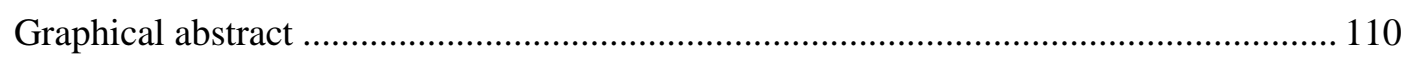

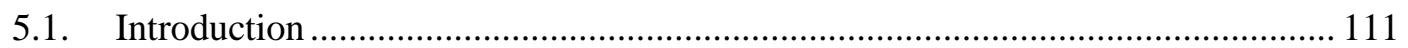

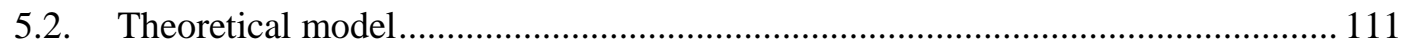

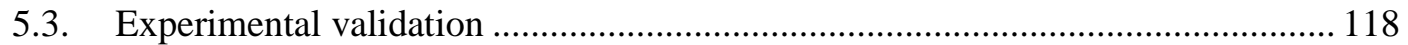




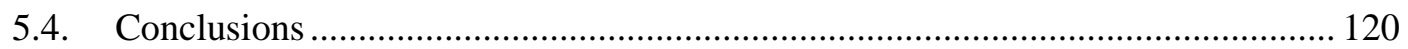

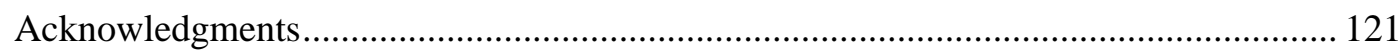

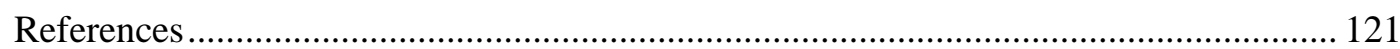

6. Characterization of thermal contacts between heat exchangers and a thermoelectric

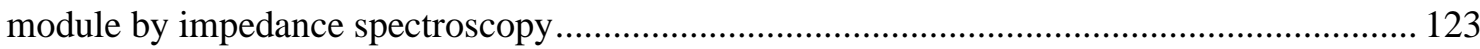

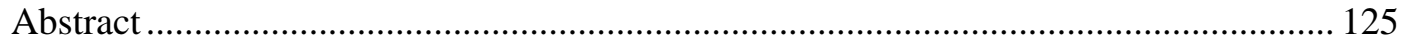

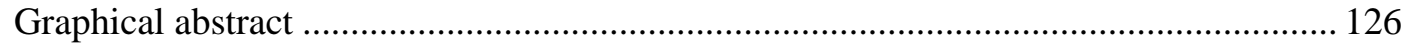

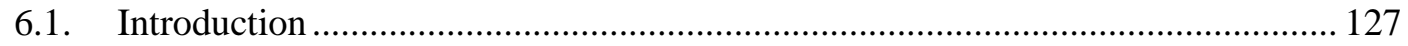

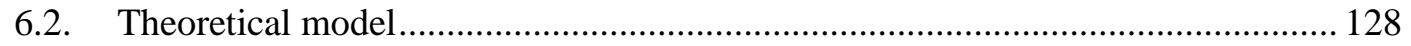

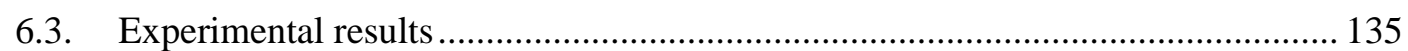

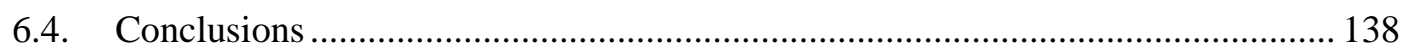

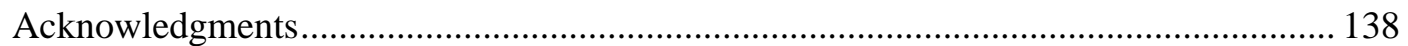

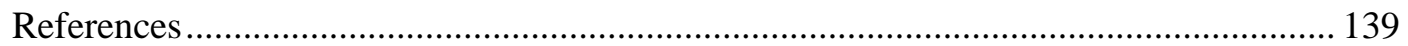

7. Detailed assessment of thermoelectric modules by a comprehensive impedance

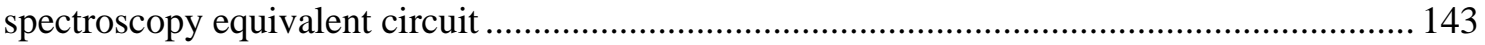

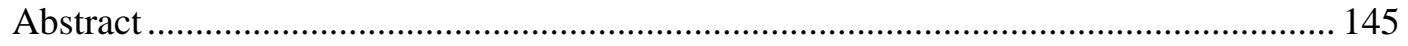

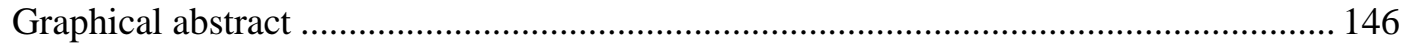

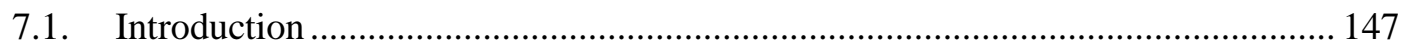

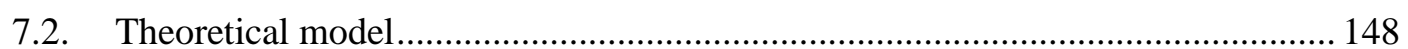

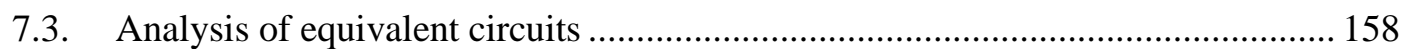

7.3.1. Thermal influence of metallic strips neglected $\left(L_{M} \rightarrow 0\right)$. Evaluation of the effect of the thermal contact resistance and $Z_{S / C}$.

7.3.2. Effect of the presence of metallic strips considering no metallic strips/ceramics thermal contact resistance

7.3.3. Effect of the presence of metallic strips considering the metallic strips/ceramics thermal contact resistance

7.3.4. All elements and effects considered ....................................................... 169

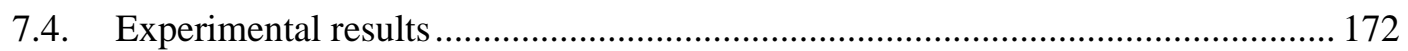

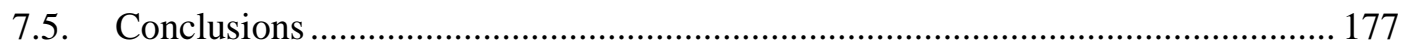

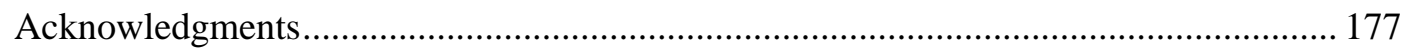

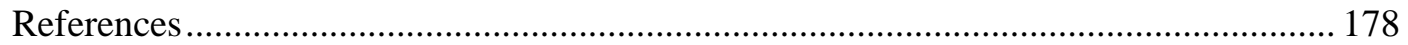




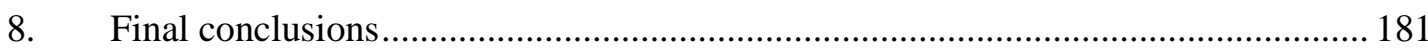

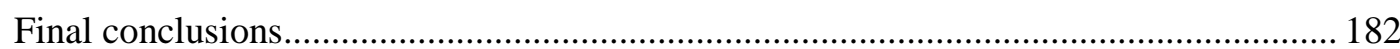

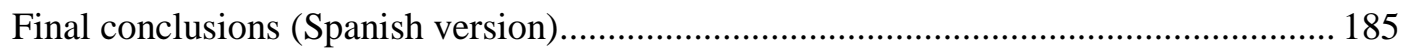

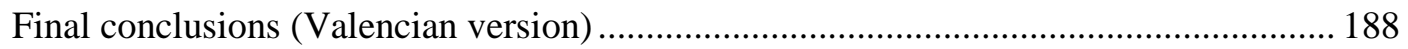

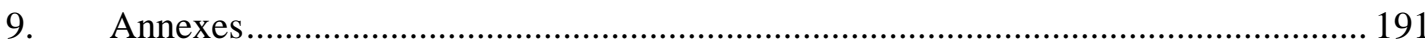

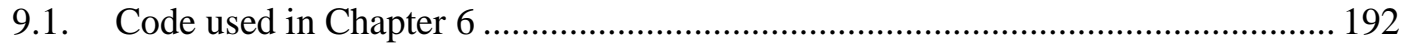

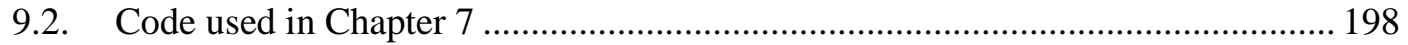

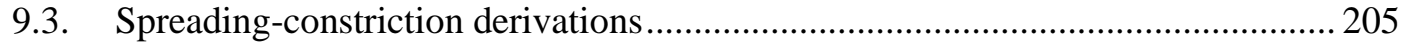

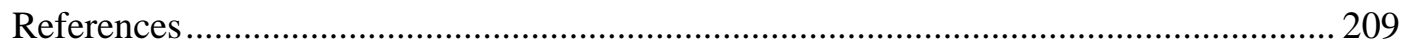

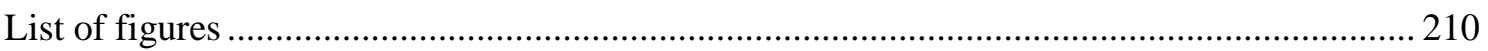

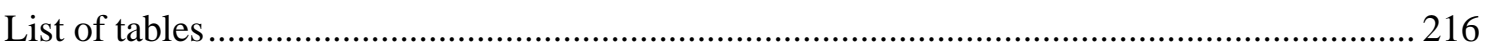


1. Introduction 


\subsection{Motivation}

The limited availability of fossil fuels, the environmental problems related to their combustion, and the ever-increasing worldwide energy demand, make the use of renewable energies and the improvement of the efficiency of current energy use an imperative need. In the past decade, it has been a strong deployment of renewable energy technologies all over the world, led by the electrical energy sector thanks to sharp cost reductions in solar photovoltaic systems and wind power[1]. However, according to the International Energy Agency, current clean energy technologies are not enough to offset the effects of an expanding global economy and growing population, leading to a slower but upwards trend in emissions, at least, until 2040 [2]. Furthermore, it is estimated that around $72 \%$ of the primary global energy is lost as waste heat [3], and $63 \%$ of that energy is lost at a temperature below $100{ }^{\circ} \mathrm{C}$, increasing the challenge of its harvesting (see Fig. 1.1).

Thermoelectric (TE) devices can directly convert heat into electricity (generation mode) or use electricity to create a temperature difference (cooling mode) under emission-free reliable operation, and without maintenance, since no moving parts are needed. Thanks to these advantages, TEs may be extremely useful for waste heat recovery (for example, in heavy-duty transportation or industries). In addition, they can also collect heat from other sources like the sun or even our own bodies. In these applications, TEs are studied to increase the efficiency of solar generators and powering wearable electronics towards achieving self-powered sensors, which can allow the elimination of batteries that currently power these devices and are subjected to frequent recharging and replacement [4,5]. Furthermore, acting as refrigerators, they are of great interest in a wide range of applications, such as cooling electronics or mobile air conditioning.

The main problem of TE materials that prevents their wide use in energy applications is their relatively low efficiency. The development of new, more efficient materials is based on the optimization of the dimensionless figure of merit $\left(z T=S^{2} \sigma T / \lambda\right)$, which is determined by three material properties: the Seebeck coefficient $(S)$, the electrical conductivity $(\sigma)$ and the thermal conductivity $(\lambda)$, and also the temperature $(T)$.

These parameters must be characterized at the temperature range of operation and their characterization usually requires the use of at least two or three different measurement 
apparatuses, which makes this task quite lengthy and tedious. On the other hand, there is no standard measurement method for this task and many times homemade equipment is used, which makes difficult to correlate measurements performed in different laboratories.
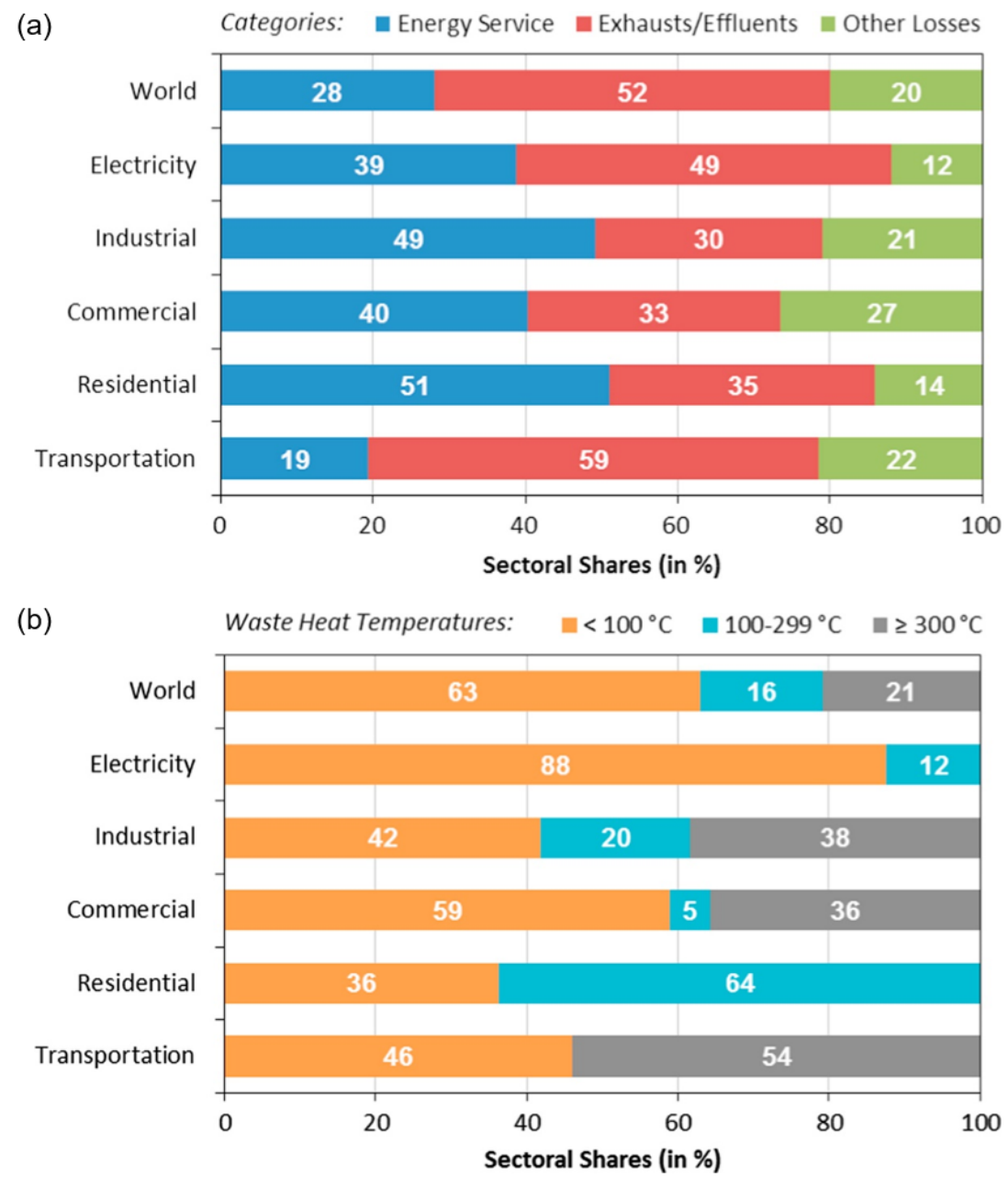

Fig. 1.1. Percentage of primary energy (a) used, wasted in exhausts/effluents, and wasted in other ways, and (b) waste heat temperatures by sector. This figure was reprinted with permission [3].

In addition, the calculation of $z T$ (efficiency) usually has a high degree of uncertainty associated, since it adds the corresponding measurement errors of the three parameters that define it ( $S, \sigma$ and $\lambda$ ). Finally, the thermal conductivity is the most difficult parameter to characterize because it usually comes with a large uncertainty, requires the use of expensive equipment that not all research groups can afford, and many of the most widely used methods to determine this 
property, such as Laser Flash, require knowledge of specific heat and density, which adds more properties to measure [6].

Impedance spectroscopy (IS) is a successfully used technique in different research areas (e.g. corrosion [7,8], fuel cells $[9,10]$, photovoltaic cells [11,12], supercapacitors $[13,14]$ or batteries [15-18]). Its success resides in its ability to separate the different processes occurring in a system, which can allow the determination of more than one parameter in a single measurement with high accuracy and, hence, being highly beneficial for the TE field. However, only a few studies showed the potential of this technique to characterize TE materials and modules [19-28] at the beginning of this thesis, and it was hardly employed in TEs. Among these previous studies, it is remarkable the report where it was shown the possibility to determine $\sigma, \lambda$ and $z T$ of TE materials using for the case of a high-performance TE material $\left(\mathrm{Bi}_{2} \mathrm{Te}_{3}\right)$. However, this study was performed only at room temperature and without a detailed analysis of its uncertainty [21].

In the work developed in this thesis, we demonstrate the capability to determine $\sigma, \lambda$ and $z T$ of TE materials by IS at temperatures up to $250^{\circ} \mathrm{C}$, for a material with modest TE properties (skutterudite), and compare the results obtained with values from commercial equipment, to quantify the precision and accuracy of the method. Moreover, we present a new method capable of determining all these properties and the Seebeck coefficient in a single IS measurement.

In this work, we also extend the ideal equivalent circuit [21] for the characterization of TE modules to include the convection effect at the outer ceramic surfaces for suspended TE modules, and the effect of the thermal contact resistance between the TE module and ideal heat sinks for sandwiched devices.

Finally, we also present a comprehensive equivalent circuit, which includes the metallic strips that connect the TE legs in a TE module, the thermal contact resistances inside the TE module (TE legs/metallic strips and metallic strips/ceramic layers), and the spreading-constriction effects.

All these developments have the potential to lead to the use of IS as the standard measurement technique for the characterization of TE materials and the assessment and quality control of TE devices (where the unavoidable addition of electrical and thermal resistances plays a crucial role). 


\subsection{Objectives}

The main objective of this thesis is to provide new developments and improvements for the advanced characterization of TE materials and devices by means of IS, in order to reach a wider implementation of this method in the TE field.

To achieve this objective, several specific objectives are proposed, covering each of them one chapter of this thesis:

- To build a setup to measure the thermal conductivity $(\lambda)$, electrical resistivity $(\sigma)$, and the dimensionless figure of merit ( $z T$ ) of $\boldsymbol{T E}$ materials with known Seebeck coefficient (S) by IS up to $250^{\circ} \mathrm{C}$, and calculate the uncertainty of the technique by comparison with commercial equipment measurements (Chapter 3).

- To build a setup to be able to measure the Seebeck coefficient in addition to the other TE properties $(\lambda, \sigma$ and $z T)$ of TE materials in a single measurement by IS up to $250{ }^{\circ} \mathrm{C}$, and calculate the errors of this approach by comparison with results from commercial equipment (Chapter 4).

- To evaluate the impact of heat convection losses at the outer ceramic surfaces in the IS response of TE modules in suspended conditions (Chapter 5).

- To study the IS response of TE modules sandwiched between perfect heat sinks and investigate the influence of the thermal contact resistances between the TE module and the heat sinks (Chapter 6).

- To develop an improved theoretical model (equivalent circuit) for IS measurements of suspended TE modules able to include all the key processes that could affect the impedance response in these devices, i.e. the influence of the metallic strips that connect the TE legs in a module, the thermal contact resistance between the TE legs and the metallic strips, and the thermal contact resistance between the metallic strips and the external insulating layers (ceramics) of the TE module (Chapter 7).

\subsection{Research strategy}

To accomplish the specific objectives proposed, two main research activities were planned,

(i) TE materials characterization and, (ii) TE modules characterization. Both research lines are 
based on the common strategy used when a system is characterized by IS, which consists in identifying or developing the theoretical model (equivalent circuit) that collects the physics of the system, and then perform a fitting, using the equivalent circuit, to the experimental IS measurements. The parameters obtained from the fittings provide the highly valuable information of the electrical and thermal properties of the TE systems.

The $\boldsymbol{T E}$ materials characterization line is divided in two parts, which correspond to Chapter 3 and Chapter 4 (see Fig. 1.2). The first part seeks the obtention of the thermal conductivity $(\lambda)$, electrical resistivity $(\sigma)$ and the dimensionless figure of merit $(z T)$, while the second part attempts to achieve a complete characterization, determining the Seebeck coefficient $(S)$ in addition to $\lambda, \sigma$ and $z T$. In both parts, the TE material is sandwiched between two metallic contacts, but the requirements of the contacts are different, high thermal conductivity for the first part and known and optimized thermal conductivity for the second. To test both approaches, first, an experimental setup capable of supporting temperatures up to $250{ }^{\circ} \mathrm{C}$ was designed. Then, the optimization of the metallic contacts for each case was necessary, including the selection of the material to stick the contacts (e.g. silver paint or liquid metals). Once the setup is ready, a sample with modest TE properties, previously measured with commercial equipment, was tested by IS and the errors were determined.

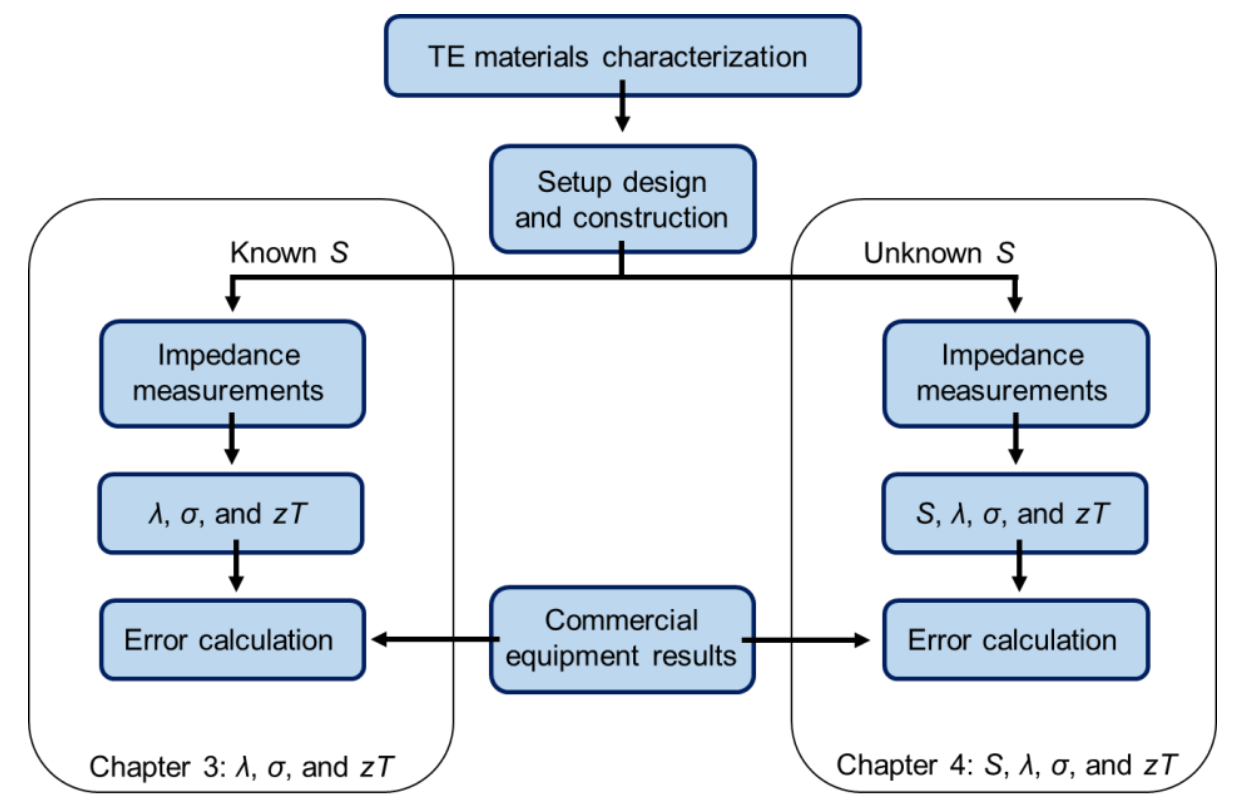

Fig. 1.2. Schematic of the research strategy followed for the characterization of thermoelectric materials. 
The $\boldsymbol{T E}$ modules characterization line is divided in three parts, which correspond to Chapters 5, 6 and 7 (see Fig. 1.3). Contrary to the TE samples characterization activities, it is necessary to develop new equivalent circuits. The new theoretical models developed for the first two parts (Chapters 5 and 6), study the convection effect at the outer ceramic surfaces of the TE modules (Chapter 5) and the effect of the thermal contact resistance of TE modules sandwiched by ideal heat sinks (Chapter 6), respectively.

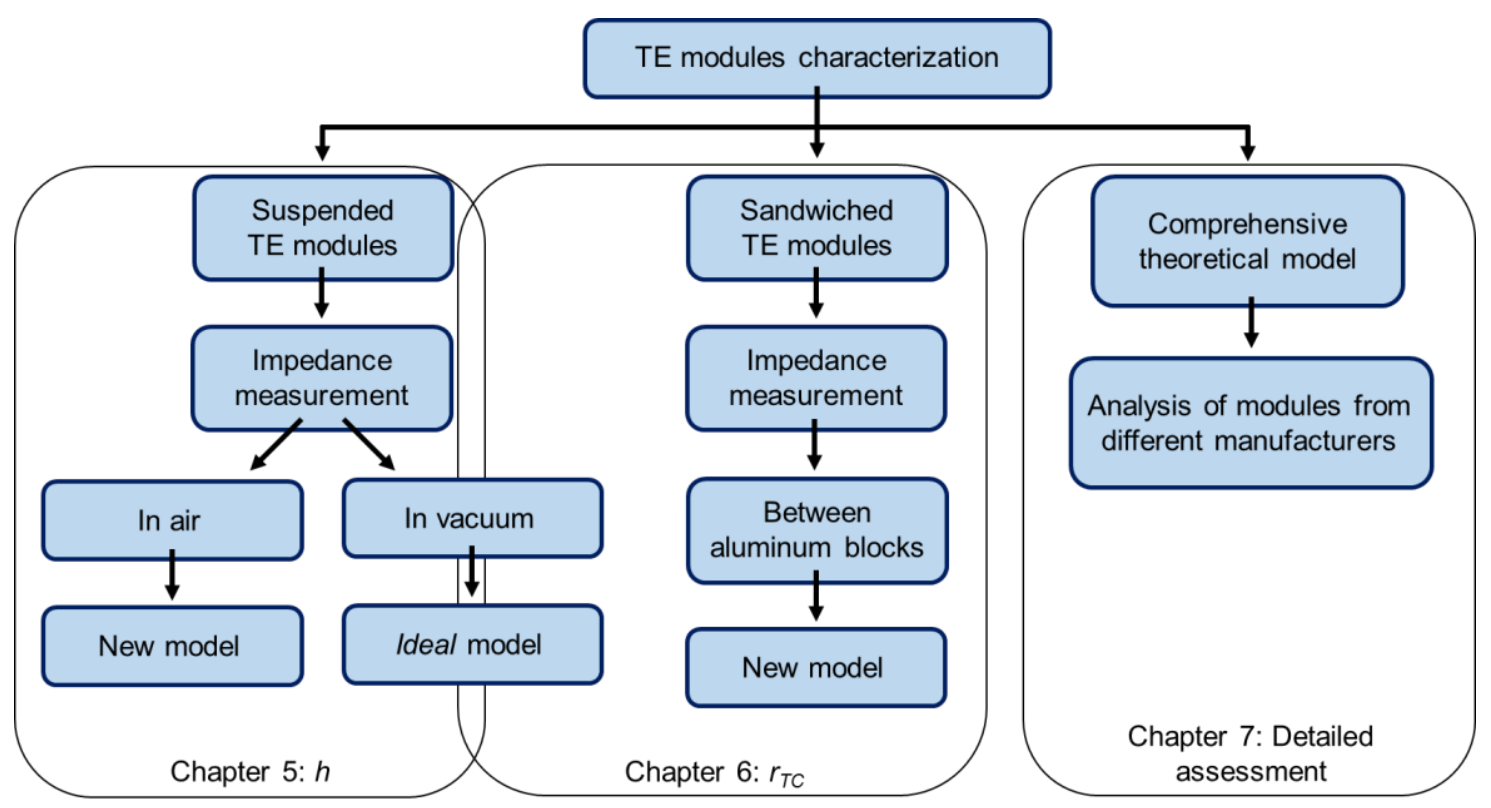

Fig. 1.3. Schematic of the research strategy followed for the characterization of thermoelectric modules.

To prove the validity of the new equivalent circuit obtained for the first part (Chapter 5), a commercial TE module was measured in suspended conditions under high vacuum (without convection) and then suspended in air to obtain the convection heat transfer coefficient value $(h)$, which is compared with reported values in the literature. For the second part (Chapter 6), a similar procedure is followed, measuring a commercial TE module suspended in vacuum and then sandwiched between large aluminum blocks to obtain the thermal contact resistance, which is also compared with previously reported values.

Finally, in the third part (Chapter 7), a new equivalent circuit including the spreadingconstriction impedance, the influence of the metallic strips, the thermal contact resistance between the TE legs and the metallic strips, and thermal contact resistance between the metallic strips and 
the ceramic layers, was developed. The addition of all these effects includes too many free variables in the equivalent circuit, making it difficult to perform fittings. However, their understanding can be used to perform a qualitative analysis of TE modules, which facilitates the detection of problems in TE devices. To explain the influence of each of the effects included in the theoretical model in the impedance measurements, simulations including all possible combinations of effects were provided. Finally, several commercial TE modules were measured, and a detailed assessment was performed.

\section{References}

[1] IRENA, International Renewable Energy Agency, Renew. Power Gener. Costs. (2018).

[2] IEA, World Energy Outlook 2019, Paris. (2019). https://www.iea.org/reports/worldenergy-outlook-2019.

[3] C. Forman, I.K. Muritala, R. Pardemann, B. Meyer, Estimating the global waste heat potential, Renew. Sustain. Energy Rev. 57 (2016) 1568-1579. https://doi.org/10.1016/j.rser.2015.12.192.

[4] A.R.M. Siddique, S. Mahmud, B. Van Heyst, A review of the state of the science on wearable thermoelectric power generators (TEGs) and their existing challenges, Renew. Sustain. Energy Rev. (2017). https://doi.org/10.1016/j.rser.2017.01.177.

[5] D. Champier, Thermoelectric generators: A review of applications, Energy Convers. Manag. 140 (2017) 167-181. https://doi.org/10.1016/j.enconman.2017.02.070.

[6] K.A. Borup, J. de Boor, H. Wang, F. Drymiotis, F. Gascoin, X. Shi, L. Chen, M.I. Fedorov, E. Müller, B.B. Iversen, G.J. Snyder, Measuring thermoelectric transport properties of materials, 8 (2015) 423-435. https://doi.org/10.1039/C4EE01320D.

[7] G.W. Walter, A review of impedance plot methods used for corrosion performance analysis of painted metals, Corros. Sci. 26 (1986) 681-703. https://doi.org/10.1016/0010938X(86)90033-8.

[8] V. Encinas-Sánchez, M.T. de Miguel, M.I. Lasanta, G. García-Martín, F.J. Pérez, Electrochemical impedance spectroscopy (EIS): An efficient technique for monitoring 
corrosion processes in molten salt environments in CSP applications, Sol. Energy Mater. Sol. Cells. 191 (2019) 157-163. https://doi.org/10.1016/j.solmat.2018.11.007.

[9] X. Yuan, H. Wang, J. Colinsun, J. Zhang, J. Colin Sun, J. Zhang, AC impedance technique in PEM fuel cell diagnosis-A review, Int. J. Hydrogen Energy. 32 (2007) 4365-4380. https://doi.org/10.1016/J.IJHYDENE.2007.05.036.

[10] S. Asghari, A. Mokmeli, M. Samavati, Study of PEM fuel cell performance by electrochemical impedance spectroscopy, in: Int. J. Hydrogen Energy, Pergamon, 2010: pp. 9283-9290. https://doi.org/10.1016/j.ijhydene.2010.03.069.

[11] F. Fabregat-Santiago, G. Garcia-Belmonte, I. Mora-Seró, J. Bisquert, Characterization of nanostructured hybrid and organic solar cells by impedance spectroscopy, Phys. Chem. Chem. Phys. 13 (2011). https://doi.org/10.1039/c0cp02249g.

[12] Z. Zolfaghari, E. Hassanabadi, D. Pitarch-Tena, S.J. Yoon, Z. Shariatinia, J. Van De Lagemaat, J.M. Luther, I. Mora-Seró, Operation Mechanism of Perovskite Quantum Dot Solar Cells Probed by Impedance Spectroscopy, ACS Energy Lett. 4 (2019) 251-258. https://doi.org/10.1021/acsenergylett.8b02157.

[13] R. Kötz, M. Hahn, R. Gallay, Temperature behavior and impedance fundamentals of supercapacitors, J. Power Sources. $154 \quad$ (2006) 550-555. https://doi.org/10.1016/J.JPOWSOUR.2005.10.048.

[14] W. Sugimoto, H. Iwata, K. Yokoshima, Y. Murakami, Y. Takasu, Proton and electron conductivity in hydrous ruthenium oxides evaluated by electrochemical impedance spectroscopy: The origin of large capacitance, J. Phys. Chem. B. 109 (2005) 7330-7338. https://doi.org/10.1021/jp044252o.

[15] R. Gopalakrishnan, Y. Li, J. Smekens, A. Barhoum, G. Van Assche, N. Omar, J. Van Mierlo, Electrochemical impedance spectroscopy characterization and parameterization of lithium nickel manganese cobalt oxide pouch cells: dependency analysis of temperature and state of charge, Ionics (Kiel). 25 (2019) 111-123. https://doi.org/10.1007/s11581-018$2595-2$.

[16] N. Vicente, M. Haro, G. Garcia-Belmonte, New approaches to the lithiation kinetics in 
reaction-limited battery electrodes through electrochemical impedance spectroscopy, Chem. Commun. 54 (2018) 1025-1040. https://doi.org/10.1039/c7cc08373d.

[17] R. Petibon, E.C. Henry, J.C. Burns, N.N. Sinha, J.R. Dahn, Comparative Study of Vinyl Ethylene Carbonate (VEC) and Vinylene Carbonate (VC) in LiCoO2/Graphite Pouch Cells Using High Precision Coulometry and Electrochemical Impedance Spectroscopy Measurements on Symmetric Cells, J. Electrochem. Soc. 161 (2014) A66-A74. https://doi.org/10.1149/2.030401jes.

[18] J. Hwang, K. Matsumoto, R. Hagiwara, Symmetric Cell Electrochemical Impedance Spectroscopy of Na2FeP2O7 Positive Electrode Material in Ionic Liquid Electrolytes, J. Phys. Chem. C. 122 (2018) 26857-26864. https://doi.org/10.1021/acs.jpcc.8b09233.

[19] S. Dilhaire, L.D. Patino-Lopez, S. Grauby, J.M. Rampnoux, S. Jorez, W. Claeys, Determination of ZT of PN thermoelectric couples by AC electrical measurement, in: Int. Conf. Thermoelectr. ICT, Proc., Institute of Electrical and Electronics Engineers Inc., 2002: pp. 321-324. https://doi.org/10.1109/ICT.2002.1190330.

[20] A.D. Downey, T.P. Hogan, B. Cook, Characterization of thermoelectric elements and devices by impedance spectroscopy, Rev. Sci. Instrum. 78 (2007) 093904. https://doi.org/10.1063/1.2775432.

[21] J. García-Cañadas, G. Min, Impedance spectroscopy models for the complete characterization of thermoelectric materials, J. Appl. Phys. 116 (2014). https://doi.org/10.1063/1.4901213.

[22] A.D. Downey, T.P. Hogan, Proceedings of the 24th International Conference on Thermoelectrics, Clemson, SC., South Carolina, USA (2005) 79-82.

[23] A.D. Downey, E. Timm, P.F.P. Poudeu, M.G. Kanatzidis, H. Shock, T.P. Hogan, Application of transmission line theory for modeling of a thermoelectric module in multiple configurations for AC electrical measurements, in: Mater. Res. Soc. Symp. Proc., Cambridge University Press, 2006: pp. 425-430. https://doi.org/10.1557/proc-0886-f1007.

[24] A. De Marchi, V. Giaretto, The Peltier driven frequency domain approach in thermal 
analysis, Rev. Sci. Instrum. 85 (2014). https://doi.org/10.1063/1.4897189.

[25] A. De Marchi, V. Giaretto, An accurate new method to measure the dimensionless figure of merit of thermoelectric devices based on the complex impedance porcupine diagram, Rev. Sci. Instrum. 82 (2011). https://doi.org/10.1063/1.3656074.

[26] A. De Marchi, V. Giaretto, S. Caron, A. Tona, A. De Marchi, V. Giaretto, S. Caron, A. Tona, A Novel zT Meter Based on the Porcupine Method and a Survey on the Size of the Snout Correction Needed for Various Thermoelectric Devices, J. Electron. Mater. 42 (2013) 2067-2072. https://doi.org/10.1007/s11664-013-2530-2.

[27] A. De Marchi, V. Giaretto, The elusive half-pole in the frequency domain transfer function of Peltier thermoelectric devices, Rev. Sci. Instrum. $82 \quad$ (2011) 034901. https://doi.org/10.1063/1.3558696.

[28] F. Casalegno, A. De Marchi, V. Giaretto, Frequency domain analysis of spreadingconstriction thermal impedance, Rev. Sci. Instrum. $84 \quad$ (2013) 024901. https://doi.org/10.1063/1.4789765. 
2. Theoretical framework 
In this chapter, we show the theoretical framework to understand the work performed in this thesis. It is divided in three parts. First, a general introduction to the thermoelectric (TE) effects is given, then, an overlook of TE materials and devices (showing their typical structure, power output, and efficiency) is performed. Finally, the basics of the impedance spectroscopy (IS) technique (which is the main technique used in this work) are presented.

\subsection{Thermoelectric effects}

Thermoelectricity is based on three effects, the Seebeck effect, which relates the potential difference that can be built in a material under a temperature difference, the Peltier effect, capable of explaining the heat absorbed/released at junctions between dissimilar conductors, and the Thompson effect. In addition, these effects are linked by two important relationships, called the Kelvin relationships. All the three effects and the Kelvin relationships are presented in this section.

\subsubsection{Seebeck effect}

Back in 1822, Thomas Johann Seebeck was the first to report this TE effect [1]. However, he thought it was a magnetic effect and it was not until a year later when Ørsted explained the physics behind this phenomenon [2]. The Seebeck effect relates to the existence of an open-circuit potential difference between both ends of an electrically conducting material when a temperature difference between those ends exists (Fig. 2.1).

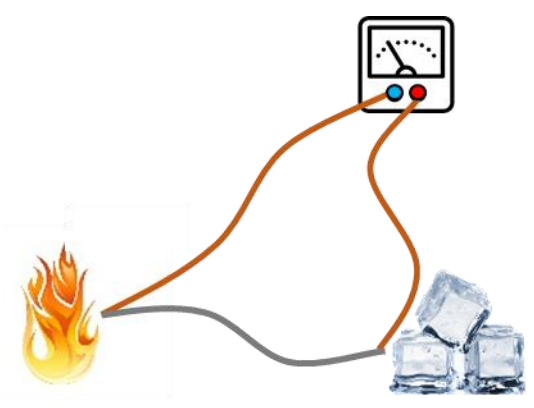

Fig. 2.1. Schematic representation of the Seebeck effect, where two different metals (brown and grey wires) produce a potential difference when exposed to a temperature difference between its junctions.

The zones of the TE material with higher temperature contain electrons which possess higher energy, allowing them to have higher mobility and, hence, their diffusion towards the zones with 
lower temperature increases. The increase in the electron diffusion induces an accumulation of positive charges in the hot side and an accumulation of negative charges in the cold side (this is the case in n-type semiconductors). The charge separation generates an electric field opposed to the thermal diffusion of the electrons (at steady state).

Eq. (2.1) shows the definition of the Seebeck coefficient $S$, which is determined by the ratio between the open-circuit potential difference ( $\triangle V$, defined as the potential of the cold side vs. the hot side) and the temperature difference ( $\Delta T=T_{H}-T_{C}$, being $T_{H}$ and $T_{C}$ the hot and cold side temperatures, respectively). Since the Seebeck coefficient depends on temperature, the temperature difference must be small for a proper estimation of $S$. Then, a larger Seebeck coefficient implies a larger potential difference for the same temperature difference.

$$
S=\lim _{\Delta T \rightarrow 0} \frac{\Delta V}{\Delta T}
$$

The Seebeck coefficient is usually expressed in $\mu \mathrm{V} / \mathrm{K}$ and its value in a good TE material lays in the $200-300 \mu \mathrm{V} / \mathrm{K}$ range. For metals, this value is typically much lower, approximately in the $1-40 \mu \mathrm{V} / \mathrm{K}$ range, which is the main reason for their lower TE performance.

It is worth noting that, by sign convention, n-type semiconductors have negative Seebeck coefficient and p-type semiconductors show positive $S$, since it is measured against the hot side and in a p-type semiconductor the free carriers are holes.

When a TE device is generating electricity (converting heat energy into electrical energy), it is said that it is working in generation mode or Seebeck mode.

\subsubsection{Peltier effect}

A few years after the discovery of the Seebeck effect, in 1834, Jean Charles Peltier discovered the Peltier effect [3]. However, he was not able to provide an explanation of this effect and it was Lenz, in 1838, who experimentally proved the heating/cooling power of this effect [4]. The Peltier effect describes the heat absorbed/released at the junctions between dissimilar conductor materials (materials with different $S$ ) due to a current flow (Fig. 2.2). The absorption and release of heat at the different junctions of the material, which produce a temperature gradient inside the TE material, is produced when the charge carriers (electrons or holes) jump from one material to the other. 


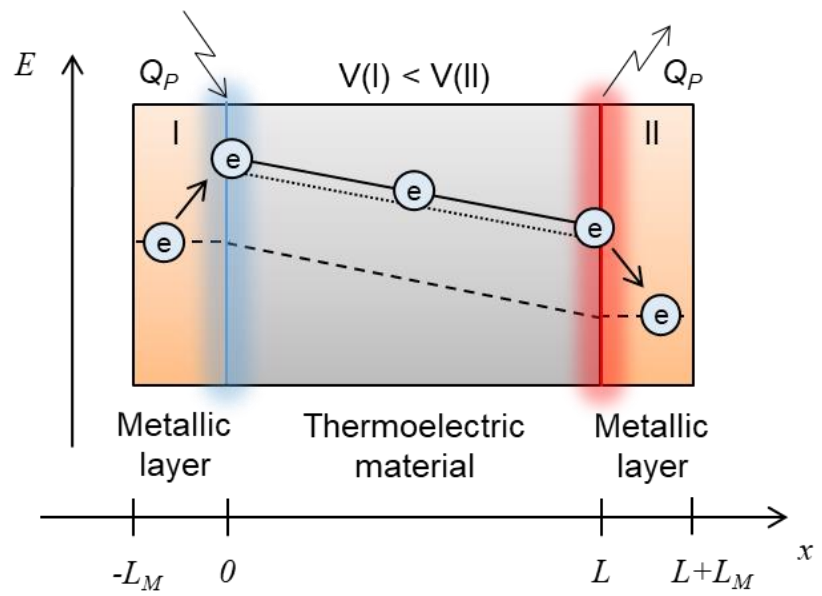

Fig. 2.2. Schematic representation of the Peltier effect. The dashed line represents the electrochemical potential, the dotted line represents the energy of the conduction band edge in the thermoelectric material, and the solid line represents the average electron energy of the electrons flowing.

Fig. 2.2 shows a semiconductor TE material contacted at both sides by metallic contacts. For the electrons to be capable of flowing inside the central conductor, it is necessary that at $x=0$ they jump to a higher energy level, absorbing heat from the lattice at this position. At the other side, at $x=L$, that energy is released to the lattice heating the junction. The heat power $Q_{P}$ generated/absorbed at both junctions is proportional to the Peltier coefficient $\Pi$, which depends on the material and the junction temperature, and the current $I$ that flows through the junction,

$$
Q_{P}=\Pi I,
$$

where the Peltier coefficient has voltage units.

It is worth noting that the current direction determines the side of the material that is heated and the side that is cooled. When a TE device uses a current to generate a temperature difference it is working as a heat pump and it is usually known as working in Peltier mode (either heating or cooling).

\subsubsection{Thomson effect}

Several years after the discovery of the Seebeck and Peltier effects, William Thomson published the third TE effect [5]. The Thomson effect consists in a uniform heat flux generated or absorbed (depending on the electrical current direction) in the whole volume of a TE material when it is subjected to both a temperature gradient and an electrical current flow. 
Unlike the Seebeck and the Peltier effect, the Thomson effect is irreversible, and it is usually negligible for low temperature gradients. The heat power absorbed or released throughout the TE material $\left(Q_{T}\right)$ is given by,

$$
Q_{T}=\beta I \Delta T,
$$

where $\beta$ is the Thomson coefficient, which has the same units than the Seebeck coefficient, V/K.

\subsubsection{Kelvin relationships}

The Kelvin relationships relate the three coefficients already presented: the Seebeck coefficient, the Peltier coefficient and the Thomson coefficient, which was also identified by William Thomson [5].

The first Kelvin relationship occurs at the junctions and relates the Seebeck coefficient and the Peltier coefficient,

$$
\Pi=S T
$$

This relationship shows that good TE materials for energy generation are also good for refrigeration. This relationship also allows the determination of one coefficient if the other is known, which is usually used to determine the Peltier coefficient from the Seebeck coefficient, since the latter is easier to determine.

The second Kelvin relationship takes effect in the whole TE material and relates the Seebeck coefficient with the Thomson coefficient,

$$
\beta=T \frac{\partial S}{\partial T}
$$

It is worth noting that knowing the Seebeck coefficient and its variation with temperature allows the rest of the coefficients to be determined.

\subsection{Materials and devices}

Thanks to their advantages (do not emit toxic gases during operation, are reliable, no maintenance is required, etc.), TE materials are gaining interest not only in refrigeration applications, where they are already being used (e.g. cooling electronics or in small portable refrigerators), but in generating electricity from wasted heat. However, this is only possible if TEs 
can generate enough power and at a suitable energy conversion efficiency. In this section, a glimpse of the most important TE materials, the typical device structure, and the power and efficiency of the TE modules, is presented.

\subsubsection{Thermoelectric materials}

In early 1900s, Altenkirch showed the characteristics that a good TE material should have for both generation and refrigeration, and introduced the concept of the figure of merit $z[6,7]$. Nowadays, it is usual to characterize TE materials using its dimensionless figure of merit $(z T)$ since this value is typically related to the energy conversion efficiency and the coefficient of performance $(C O P)$, and it is an indicator of its performance. The efficiency and $C O P$ of a TE material increases when the $z T$ value increases too, thus, high values of this parameter are convenient. The $z T$ is defined as,

$$
z T=\frac{S^{2} \sigma}{\lambda} T,
$$

where $\sigma$ is the electrical conductivity, $\lambda$ the thermal conductivity, and $T$ the temperature.

The research on new TE materials pursues finding higher values of $z T$. A higher value of the Seebeck coefficient provides higher open-circuit voltage for the same temperature gradient, a higher electrical conductivity allows higher values of current flow, and a small thermal conductivity increases the temperature difference between the material sides for the same heat flow, increasing the efficiency of the TE material. However, increasing $z T$ is a considerable challenge because the optimization of one of the parameters that govern $z T$ often adversely affects the others, as shown in Fig. 2.3 [8].

All these parameters strongly depend on the concentration of charge carriers in the TE material. Fig. 2.3 shows schematically the evolution of the Seebeck coefficient, electrical conductivity, thermal conductivity, and $z T$ for different charge carrier concentrations. For small charge carrier concentrations, the Seebeck coefficient is high and the thermal conductivity is low, however, the electrical conductivity is very low, and it produces low values of $z T$. For large charge carrier concentrations, the Seebeck coefficient strongly decreases (also the thermal conductivity increases but its effect is less prominent) and, even though the electrical conductivity increases, the $z T$ of the material is also low. Then, the highest values of the dimensionless figure of merit 
are obtained for intermediate values of charge carrier concentrations, usually between $10^{19}$ and $10^{21} \mathrm{~cm}^{-3}$.

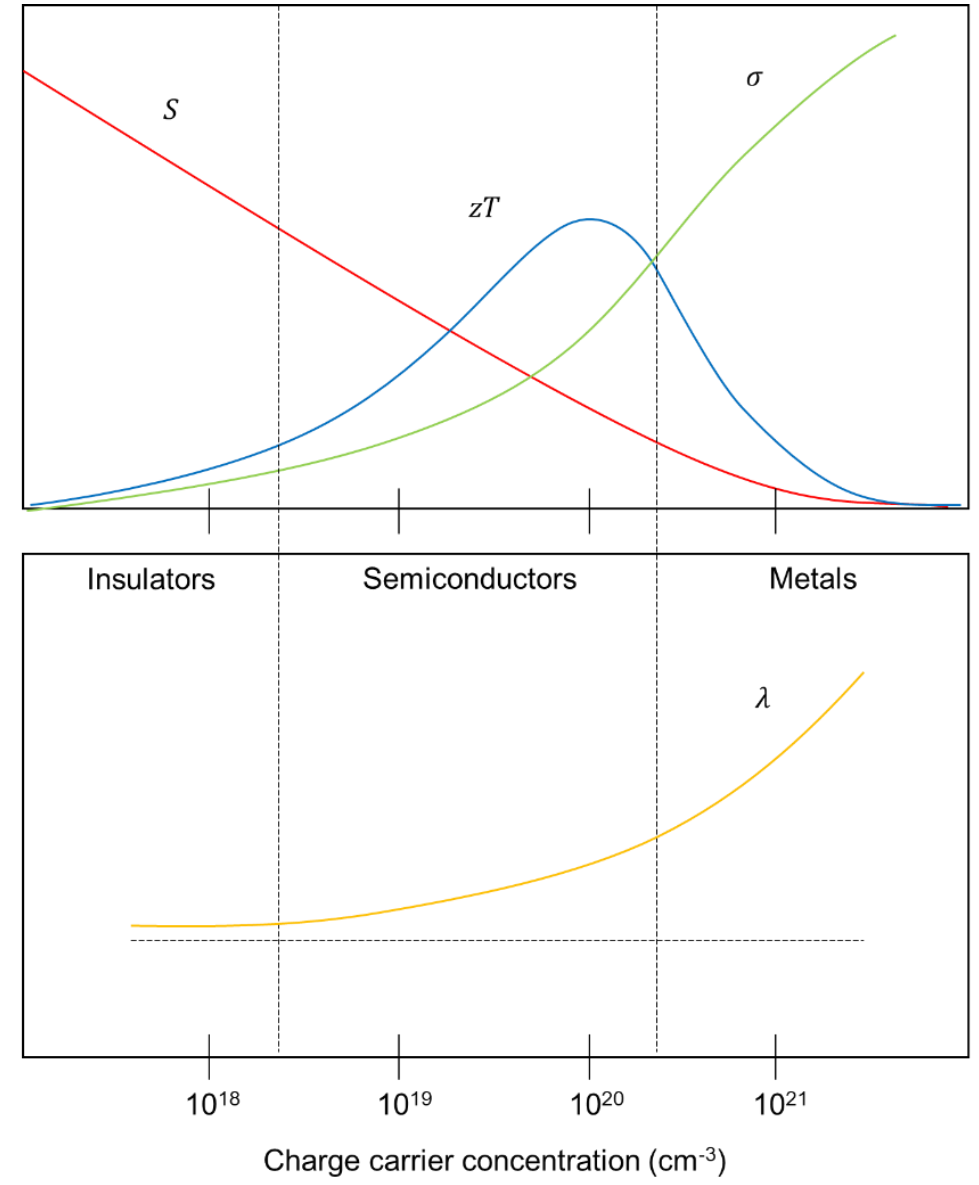

Fig. 2.3. Schematic representation of the influence of the charge carrier concentration in the Seebeck coefficient (red), electrical conductivity (green), $z T$ (blue), and thermal conductivity (orange). The horizontal dashed line represents the lattice thermal conductivity.

While metals have a large number of charge carriers, insulators very few, so, neither of both are suitable TE materials. Then, the most suitable quantity of charge carriers is obtained in heavily doped semiconductors. This theory was presented for the first time back in 1931 by Abram Ioffe, when he foresaw the significant potential of semiconductor materials in the TE field [9]. Not all semiconductors perform in the same way, hence, the most suitable TE material depends on the temperature range of operation. For example, Bi-Te alloys are good TE materials near room temperature but do not hold high temperatures. On the other hand, skutterudites present low 
performance at low temperatures, but it increases with temperature. Right now, some of the most important families of TE materials are Bi-Te alloys, skutterudites, Half-Heusler compounds, alloys based on SnSe, and tetrahedrite compounds. In recent years, a lot of progress increasing the $z T$ has been made, indicating that this situation may change in the near future due to the emergence of new TE materials families (see Fig. 2.4).
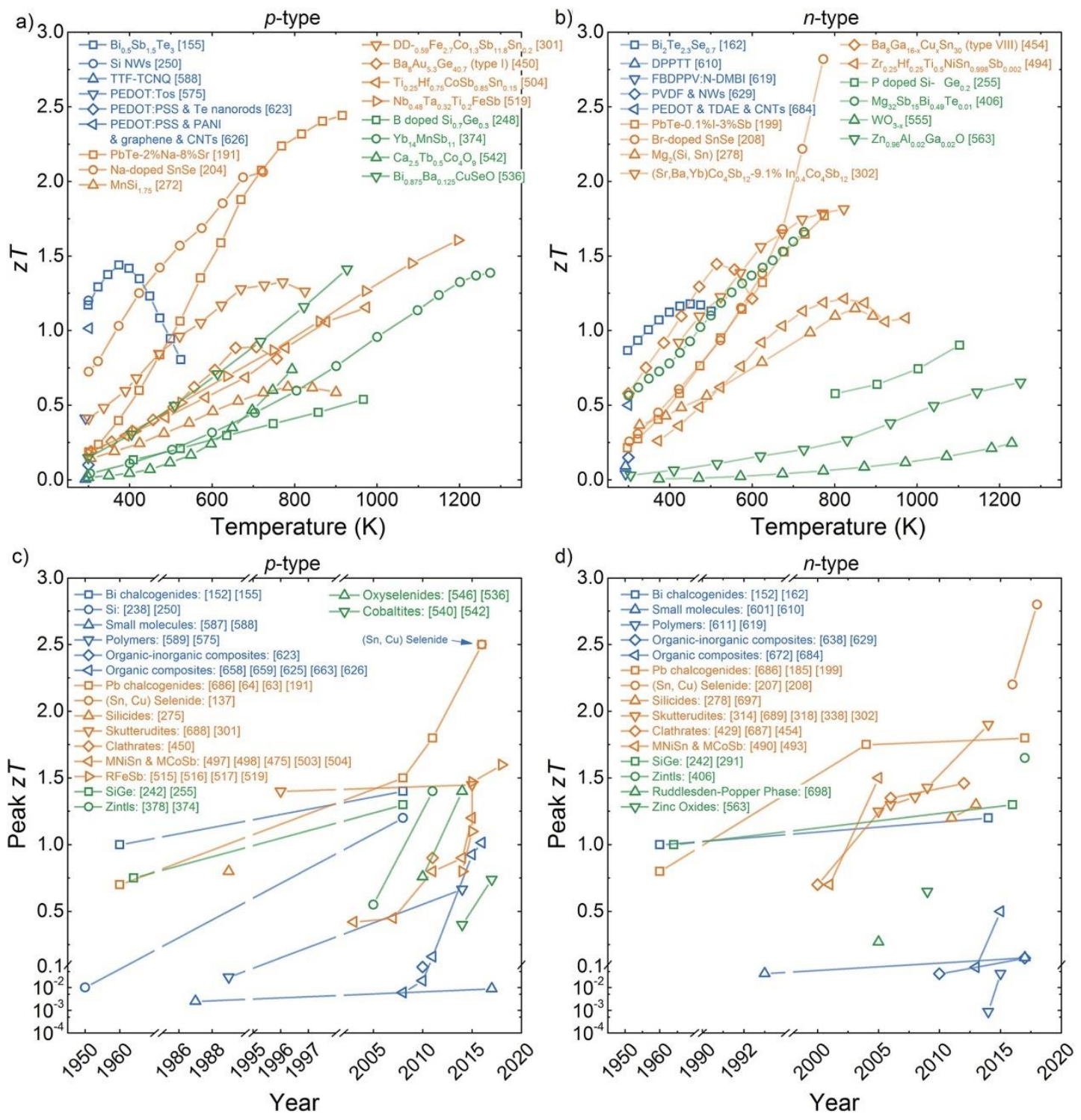

Fig. 2.4. Material's $z T$ as a function of temperature for the most common (a) p-type and (b) n-type thermoelectric materials, and (c, d) its peak evolution over the years, respectively. This figure was reprinted with permission [10]. 


\section{Bismuth telluride alloys}

Bismuth telluride is the most commercially used TE material for near room temperature applications, where $z T$ values slightly higher than 1 can be found for both p-type and n-type materials $[11,12]$. In recent years, nanostructuring has shown to be effective in improving the $z T$ of some materials, as in Bi-Te alloys [13]. For example, a p-type nanostructured $\mathrm{Bi}_{0.5} \mathrm{Sb}_{1.5} \mathrm{Te}_{3}$ material has been reported to present the highest $z T$ in this material family $(1.86$ at $320 \mathrm{~K})$ [14]. However, the production of nanostructured materials at a large scale present difficulties.

\section{$\underline{\text { Skutterudites }}$}

Skutterudites are based on $\mathrm{CoSb}_{3}$, contain small amounts of other elements and they are considered as one of the most promising TE materials, mainly due to its relatively low cost (see Fig. 2.5) [15]. These TE materials are suitable for medium temperature applications since they usually have the $z T$ peak between $400{ }^{\circ} \mathrm{C}$ and $550^{\circ} \mathrm{C}$. P-type skutterudites can reach $z T$ values of 1.3 whereas n-type skutterudites can surpass $z T$ values of 1.8 [15].

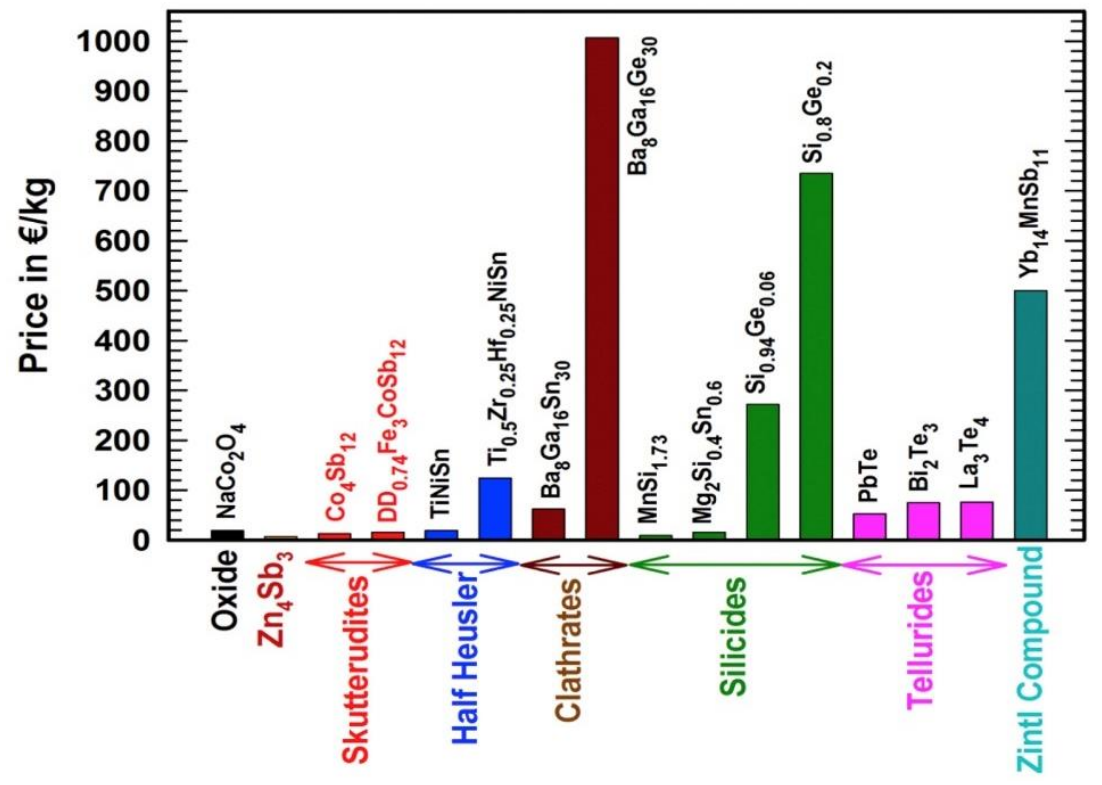

Fig. 2.5. Price of the constituents for some of the most common thermoelectric materials (2016). This figure was reprinted with permission [15]. 


\section{$\underline{\text { Half-Heusler }}$}

Half-Heusler alloys are intermetallic compounds with formula MNiSn (M=Ti, Hf, Zr). They have their maximum $z T$ values at temperatures between $650{ }^{\circ} \mathrm{C}$ and $800{ }^{\circ} \mathrm{C}$ and can withstand up to $1000{ }^{\circ} \mathrm{C}$. In recent years, peak $z T$ values higher than 1 have been achieved for both $\mathrm{n}$-type and p-type Half-Heusler materials [16-18].

\section{$\underline{\text { Tin selenide }}$}

Tin selenide ( $\mathrm{SnSe}$ ) started to gain interest in the TE field when an unprecedented $z T$ of 2.6 at $650{ }^{\circ} \mathrm{C}$ was reported in 2014 [19]. Doping SnSe with other elements such as Na or Bi has shown improved TE properties at lower temperatures. However, the techniques used to produce them were expensive, lengthy, and require high temperatures, which are not favorable for commercial use [20]. More recently, in 2018, a peak $z T$ value of 2.8 at $500{ }^{\circ} \mathrm{C}$ was obtained for an n-type $\mathrm{SnSe}$ doped with bromine, one of the highest peak $z T$ ever reported [21].

\section{Tetrahedrites}

Tetrahedrite materials (based on $\mathrm{Cu}_{12} \mathrm{Sb}_{4} \mathrm{~S}_{13}$ ) are light, non-toxic, earth-abundant, cheap, and show relatively good TE properties. Due to all these advantages, they have the potential to be competitive with commercial TE materials [22]. Peak $z T$ values for tetrahedrite materials are currently over $1[23,24]$.

\subsubsection{Thermoelectric devices}

The usual configuration of a TE device (also known as TE module or Peltier device) is the electrical connection in series and thermal connection in parallel of TE legs alternating p-type and n-type materials (see Fig. 2.6).

All the TE legs are connected by metal strips (usually, copper) and supported on its upper and lower parts by two layers of an electrically insulating material with the highest possible thermal conduction (typically, a ceramic material such as alumina), as can be seen in Fig. 2.6.

For both applications (refrigeration and energy generation), the power of TE device can be modified by varying its geometry and number of TE elements. Since the legs of materials are electrically connected in series, the module voltage is the sum of the contribution of all the 
elements. It is common to find TE devices with more than 100 pairs of legs (a pair is the junction of a p-type leg and a n-type leg).

(a)

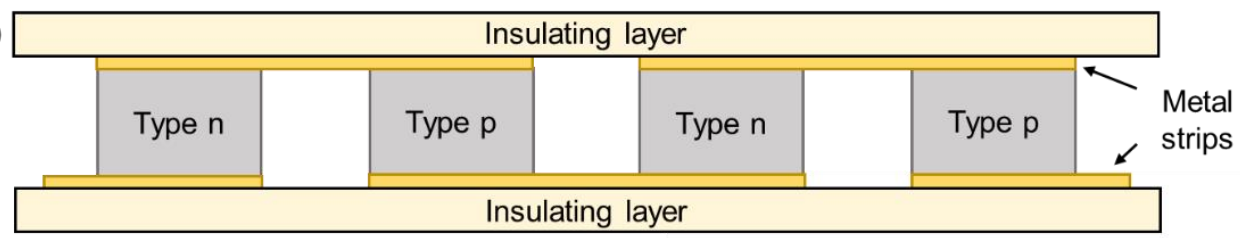

(b)

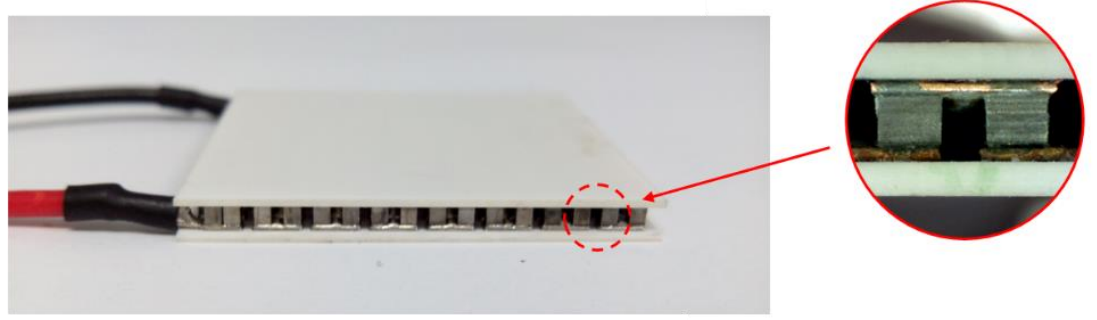

Fig. 2.6. (a) Schematic view of the typical structure of a thermoelectric device, and (b) an actual picture of a commercial Bi-Te device with a magnification of a pair.

\subsubsection{Power output of thermoelectric devices}

When a TE device is working in power generation mode, it generates electricity from a temperature difference and current is supplied to an electric load. The potential difference at the load $\left(\Delta V_{\text {load }}\right)$ at any moment is given by,

$$
\Delta V_{\text {load }}=I R_{\text {load }}=S_{m} \Delta T-I R_{\Omega}
$$

where $S_{m}$ is the Seebeck coefficient of the device, $\Delta T=T_{H}-T_{C}$ is the temperature difference between both sides of the TE materials, $R_{\Omega}$ is the total ohmic resistance of the module, $R_{\text {load }}$ is the resistance of the load connected, and $I$ is the current generated by the device.

From Eq. (2.7), we can find the current generated by the device,

$$
I=\frac{S_{m} \Delta T}{R_{\Omega}+R_{\text {load }}} .
$$

Knowing the voltage difference across the load and the current that circulates through it, the power extracted $P_{\text {out }}$ from the TE device can be determined by multiplying Eq. (2.7) and Eq. (2.8),

$$
P_{\text {out }}=\Delta V_{\text {load }} I=\frac{\left(S_{m} \Delta T\right)^{2} R_{\text {load }}}{\left(R_{\Omega}+R_{\text {load }}\right)^{2}} .
$$


The maximum power is obtained when the total ohmic resistance of the TE module is equal to the load resistance that is connected, in that case, the power is,

$$
P_{\text {Max }}=\frac{\left(S_{m} \Delta T\right)^{2}}{4 R_{\Omega}}
$$

This equation shows that high Seebeck coefficient and low electrical resistivity provide high maximum power output.

\subsubsection{Efficiency of thermoelectric devices}

The efficiency $\eta_{m}$ of TE devices for energy generation applications is calculated from the heat power input that reaches the TE module $Q_{i n}$, which comes from the heat source, and the electrical power output of the module [Eq. (2.9)],

$$
\eta_{m}=\frac{P_{\text {out }}}{Q_{\text {in }}}
$$

From the energy balance at the hot side of the TE legs, and neglecting the ceramic layers and the electrical contacts, $Q_{i n}$ can be obtained at steady state,

$$
Q_{\text {in }}=S_{m} T_{H} I-\frac{1}{2} I^{2} R_{\Omega}+K_{m}\left(T_{H}-T_{C}\right)
$$

where $K_{m}$ is the total thermal conductance of the TE device, and $T_{H}$ and $T_{C}$ are the hot and cold side temperatures, respectively.

Eq. (2.12) contains three terms, the first of them refers to the heat power that is removed from all the hot junctions of the TE module by the Peltier effect, the second corresponds to the variation of the heat power conduction toward the TE legs induced by the Joule effect, and the third one is the heat power conduction through the TE legs. Introducing Eq. (2.9) and Eq. (2.12) into Eq. (2.11) and deriving with respect to $R_{\text {load }}$, the expression that defines the maximum efficiency can be obtained [4],

$$
\eta_{\text {Max }}=\left(\frac{T_{H}-T_{C}}{T_{H}}\right) \frac{\sqrt{1+Z_{m} \bar{T}}-1}{\sqrt{1+Z_{m} \bar{T}}+\frac{T_{C}}{T_{H}}}
$$

being $\bar{T}$ the average temperature between the hot side $\left(T_{H}\right)$ and the cold side $\left(T_{C}\right)$ of the TE materials, and $Z_{m}$ the figure of merit of the TE module, which is defined as, 


$$
Z_{m}=\frac{S_{m}{ }^{2}}{R_{\Omega} K_{m}} .
$$

Looking at Eq. (2.14), the figure of merit of the module has units of $\mathrm{K}^{-1}$, although it is usual to define the dimensionless figure of merit $Z_{m} T$, multiplying by the temperature, as described in Eq. (2.6) for TE legs. It is worth noting that the parameters in Eq. (2.14) refer to module properties whereas the parameters in Eq. (2.6) refer to materials properties. The $Z_{m} T$ of the modules is always lower than the $z T$ of its materials due to the introduction of the resistance of the metallic strips and the electrical contact resistances when the module is fabricated.

Fig. 2.7 shows simulations of the theoretical efficiency that TE modules with different $Z_{m} T$ values would achieve at different hot side temperatures when the cold side is maintained at 300 $\mathrm{K}$. These simulations are performed using Eq. (2.13), which is obtained considering all the TE properties temperature independent, and neglecting thermal resistances, which are quite significant in the design of TE systems [25]. As it can be seen, to achieve efficiencies comparable to conventional mechanical generators $(\approx 30 \%$ for $\Delta T \approx 500 \mathrm{~K}), Z_{m} T$ values over 3 are necessary $[26,27]$.

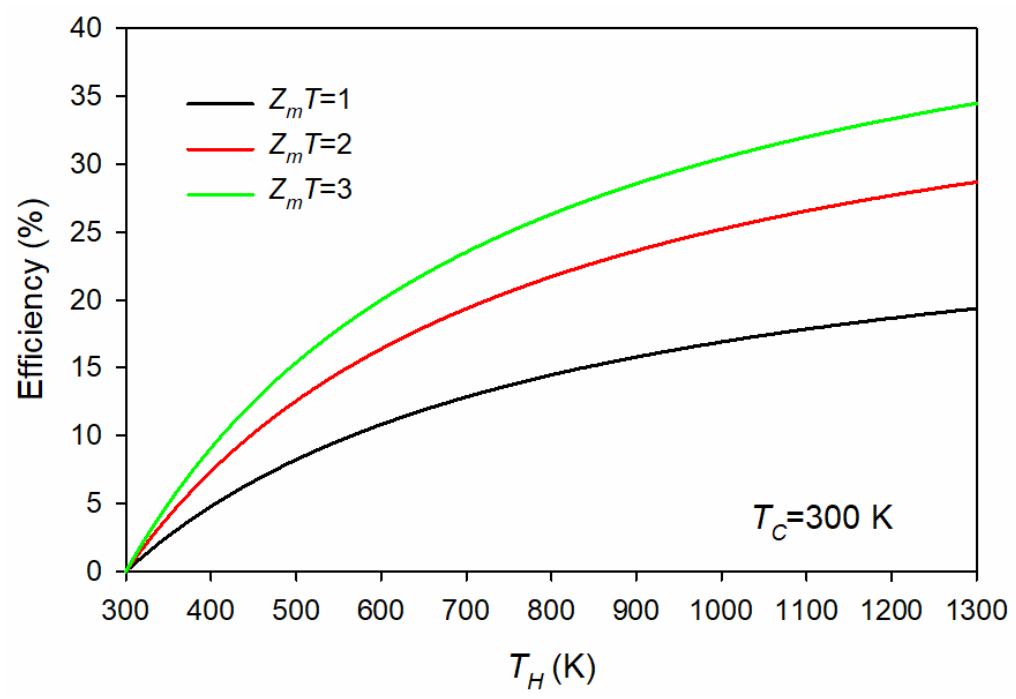

Fig. 2.7. Theoretical heat-to-electricity energy conversion efficiency for thermoelectric modules with different $Z_{m} T$ values as a function of the hot side temperature, maintaining the cold side temperature at 300 $\mathrm{K}$. The simulations are made using Eq. (2.13).

It is worth keeping in mind that the $z T$ of any TE material depends on temperature, then, depending on the application in which it is being used, one material may be more suitable than 
another. For example, some materials have modest TE properties at low temperature, but they improve greatly with temperature. Others, on the other hand, at moderate temperatures have acceptable properties but are not able to withstand higher temperatures because they are already approaching their melting point. In addition, the TE materials in some real applications are exposed to a large temperature gradient so, the $z T$ of the material may change significantly throughout its length, increasing the importance of having a large range with a high $z T$ over having a larger $z T$ peak [28].

Similar to the efficiency of TE modules for generation applications, the coefficient of performance of a TE module is defined for cooling $\left(C O P_{c}\right)$ and heating $\left(C O P_{h}\right)$ applications as,

$$
\begin{aligned}
& C O P_{c}=\frac{Q_{\text {out }, c}}{P_{\text {in }}} \\
& \operatorname{COP}_{h}=\frac{Q_{\text {out }, h}}{P_{\text {in }}}
\end{aligned}
$$

being $Q_{o u t, c}$ the heat power extracted from the cold side, $Q_{\text {out }, h}$ the heat power generated at the hot side, and $P_{\text {in }}$ the electrical power supplied to the TE device, which are given by,

$$
\begin{aligned}
& P_{\text {in }}=S_{m} I \Delta T-I^{2} R_{\Omega}, \\
& Q_{o u t, c}=S_{m} T_{C} I-\frac{1}{2} I^{2} R_{\Omega}-K_{m}\left(T_{H}-T_{C}\right) \\
& Q_{o u t, h}=S_{m} T_{H} I+\frac{1}{2} I^{2} R_{\Omega}-K_{m}\left(T_{H}-T_{C}\right)
\end{aligned}
$$

Finally, introducing Eq. (2.17), Eq. (2.18) and Eq. (2.19) into Eq. (2.15) and Eq. (2.16), an expression for both $C O P$ can be obtained [29-31],

$$
\begin{aligned}
& \operatorname{COP}_{c}=\left(\frac{T_{C}}{T_{H}-T_{C}}\right) \frac{\sqrt{1+Z_{m} \bar{T}}-\frac{T_{H}}{T_{C}}}{\sqrt{1+Z_{m} \bar{T}}+1}, \\
& \operatorname{COP}_{h}=\left(\frac{T_{H}}{T_{H}-T_{C}}\right)\left(1-2 \frac{\sqrt{1+Z_{m} \bar{T}}-1}{Z_{m} \bar{T}}\right) .
\end{aligned}
$$

Fig. 2.8(a) shows simulations of the COP for cooling applications that TE modules with different $Z_{m} T$ values would achieve at different cold side temperatures when the hot side is maintained at 300 K. Similarly, Fig. 2.8(b) shows the simulations of the COP for heating applications at different hot side temperatures when the cold side is maintained at $300 \mathrm{~K}$. Both 
Chapter 2

figures were obtained with Eq. (2.20) and Eq. (2.21), respectively. As expected, both curves show a similar trend, obtaining the highest $C O P$ values for low temperature difference, being even higher for heating than for cooling applications.
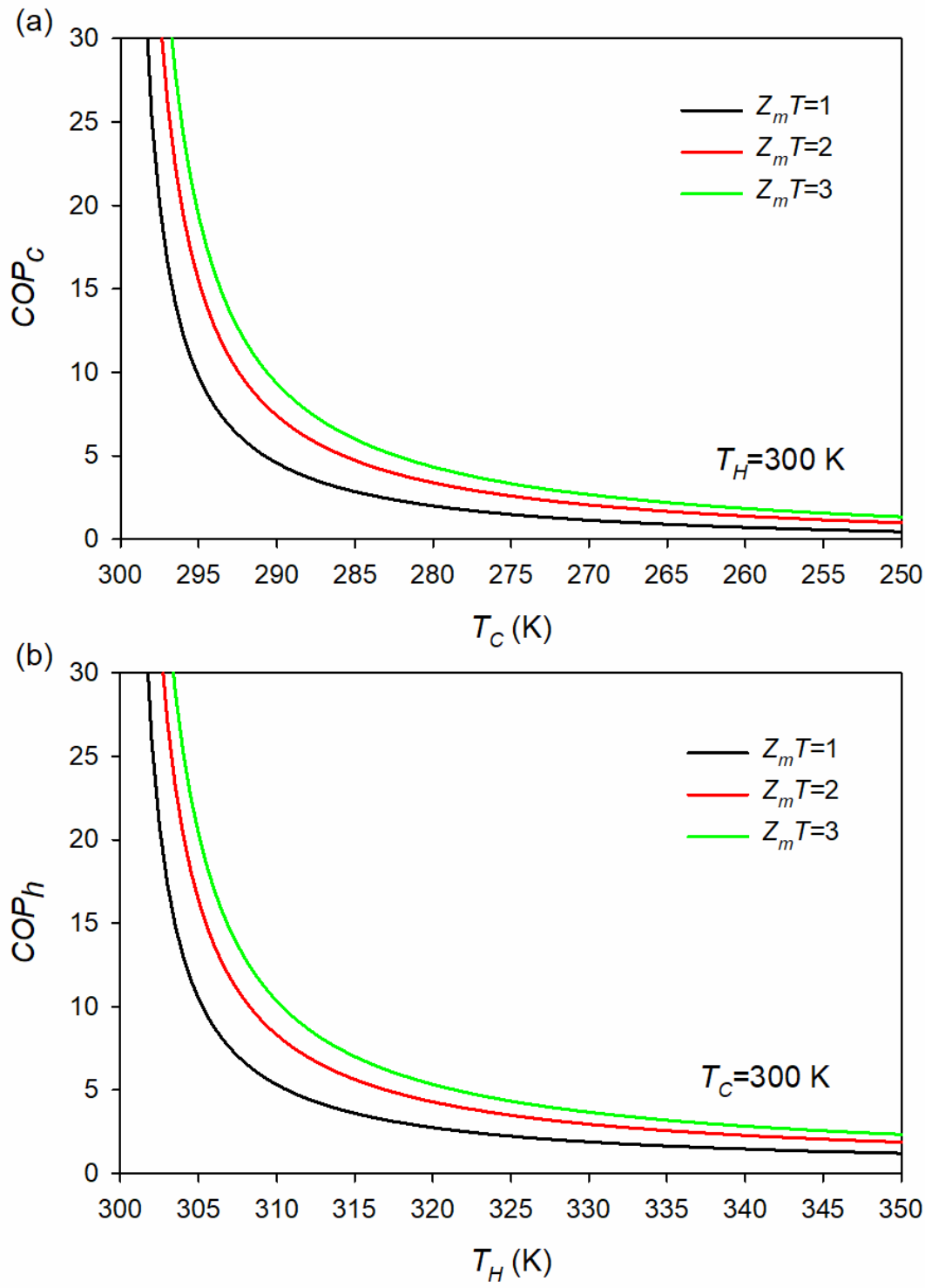

Fig. 2.8. Theoretical coefficient of performance of thermoelectric modules with different $Z_{m} T$ values for (a) cooling and (b) heating applications. The simulations are made using Eq. (2.20) and Eq. (2.21), respectively. 


\subsection{Impedance spectroscopy technique}

In this section, the basics of the impedance method, which is the technique that is developed in this thesis, and the initial theoretical models (equivalent circuits) obtained for its use in TE characterization are presented.

\subsubsection{Introduction to the technique}

IS is a widely used technique in many fields. This widespread use is due to the remarkable characteristics that this method offers. Among them, there are very reliable and precise commercial equipment available in the market, it usually allows the separation of the processes that occur in the devices, and it can be used under working conditions.

The technique consists in the application of a low amplitude sine wave signal (which can be current or voltage) around a fixed (dc) value of this signal at a certain frequency. Due to its low amplitude, there will be a proportionality between voltage and current. Hence, the application of a sinusoidal current of certain amplitude $I_{a c}$ will generate a proportional sinusoidal voltage with its corresponding amplitude $V_{a c}$, which may be displaced by a certain phase value $\varphi$ (see Fig. 2.9).

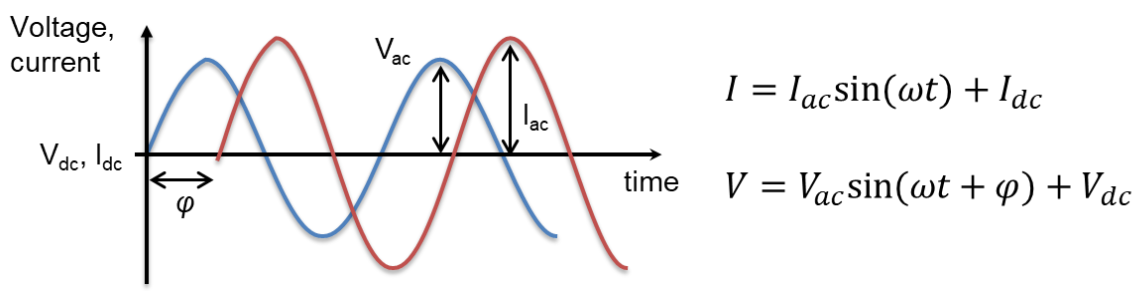

Fig. 2.9. Signals applied and recorded during an impedance spectroscopy measurement, voltage (blue), and current (red) at a certain frequency. The equations represent the current $(I)$ and voltage $(V)$ sinusoidal signals for an angular frequency ( $\omega=2 \pi f$, being $f$ the frequency) as a function of time $t$.

The impedance signal is represented by a point in the complex plane, defined by a vector with certain module and angle [see the arrow in Fig. 2.10(a)]. The module of the vector $|Z|$ is calculated by the ratio of the voltage and current amplitudes,

$$
|Z|=\frac{V_{a c}}{I_{a c}}
$$

and the vector angle is given by the phase difference between the two waves. 

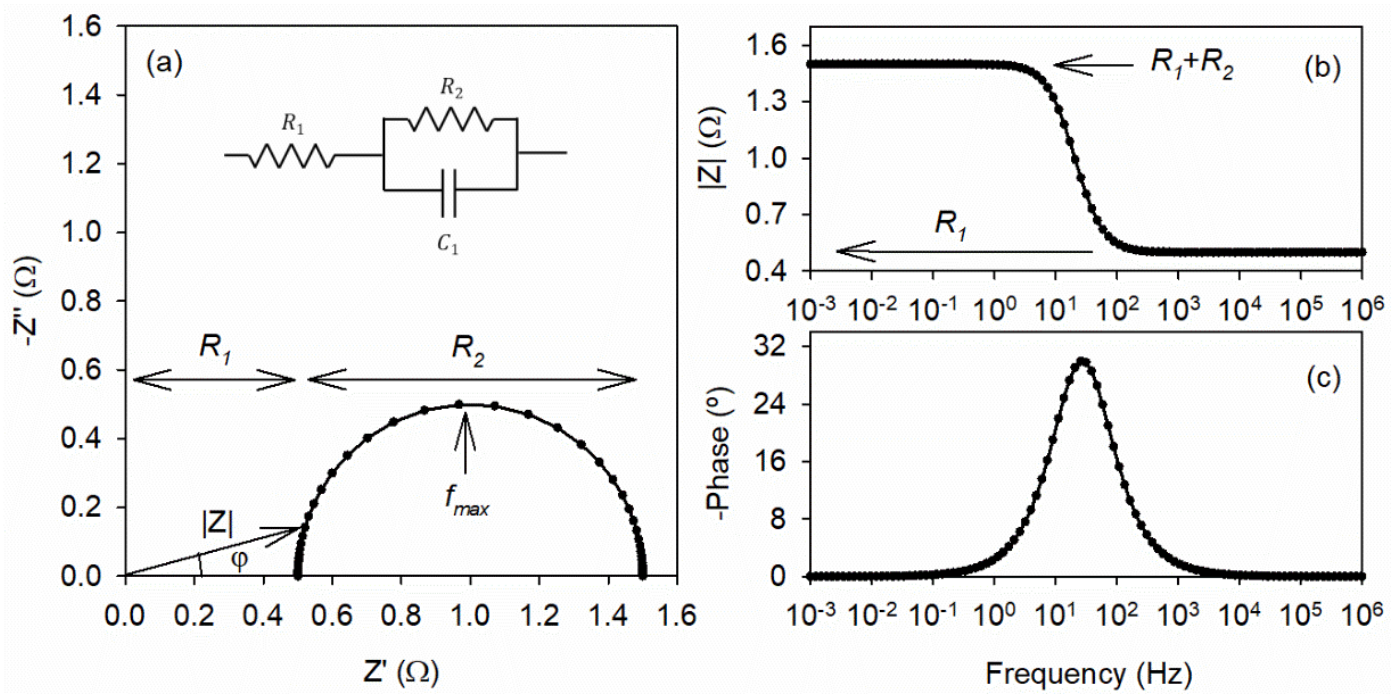

Fig. 2.10. Simulations from $1 \mathrm{mHz}$ to $1 \mathrm{MHz}$ of an impedance spectrum represented in a Nyquist plot (a) and Bode plots (b) and (c). The inset shows the equivalent circuit used to generate the data. For the simulations $R_{l}=0.5 \Omega, R_{2}=1 \Omega$, and $C_{l}=0.01 \mathrm{~F}$ were used. Frequency decreases from left to right in (a).

The entire spectrum described in Fig. 2.10 is obtained by repeating the test in Fig. 2.9 for a wide range of different frequency values, showing different points in the complex plane (one point for each frequency). Normally, frequency ranges between $10 \mathrm{mHz}$ and $1 \mathrm{MHz}$ are used, with a total of 40 to 60 logarithmically distributed frequencies. The frequency range is usually chosen to obtain 3 or 4 points after the impedance peak and lower frequency values are not used to avoid an unnecessary large measuring time. Quick processes appear at high frequencies and slow processes are shown at low frequencies, which can allow the characterization of more than one parameter in a single measurement. The impedance results are commonly presented in two ways, by Nyquist plots [in the complex plane, Fig. 2.10(a)] or by Bode plots [Fig. 2.10(b) and Fig. 2.10(c)], which include the variation of different parameters, such as the magnitude of the impedance or the value of the phase, with frequency.

The usual procedure to analyze IS measurements involves the use of equivalent circuits, which are constructed from the physics of the system. For example, the impedance of the equivalent circuit shown in the inset of Fig. 2.10(a), $Z_{e x}$, is the series summation of a resistor $\left(R_{l}\right)$ with the parallel combination of another resistor $\left(R_{2}\right)$ and a capacitor $\left(C_{l}\right)$,

$$
Z_{\text {ex }}=R_{1}+\left(R_{2}^{-1}+j \omega C_{1}\right)^{-1},
$$


being $j=(-1)^{0.5}$ the imaginary number. Note that $Z_{C}=\left(j \omega C_{l}\right)^{-1}$ is the impedance of a capacitor. Then, at high frequencies, the impedance of $C_{l}$ vanishes and the response is only driven by $R_{l}$. However, at low frequencies the magnitude of $C_{l}$ increases until its own influence becomes negligible and the response is governed by the series combination of both resistors $\left(R_{1}+R_{2}\right)$. At intermediate frequencies the capacitor plays and important role, creating a semicircle with a minimum in the imaginary part of the impedance (maximum in $-Z^{\prime \prime}$ ) at a frequency $f_{\max }=1 /\left(2 \pi R_{2} C_{l}\right)$. Once the experimental impedance spectrum is measured, a fitting to the equivalent circuit of the system under evaluation is typically performed to obtain the parameters that define that system [32]. For example, a fitting with the equivalent circuit shown in the inset of Fig. 2.10(a) provides the values of $R_{l}, R_{2}$ and $C_{l}$ from the high frequency interception, the diameter of the semicircle and the value of $f_{\max }$, respectively (see Fig. 2.10). For a correct interpretation of the impedance spectra, it is necessary that all the elements in the equivalent circuit hold a physical meaning, since more than one equivalent circuit may produce a suitable fitting.

\subsubsection{Impedance spectroscopy in thermoelectricity}

To our knowledge, the first reported use of IS in thermoelectricity dates back from 2002, and showed the idea of obtaining the $Z_{m} T$ of TE devices in a single p-n junction [33]. Later, in 2005, the first models for the characterization of single TE legs, unicouples, and inline TE modules were developed based on transmission line models and $R C$ circuits [34-36]. Starting in 2011, De Marchi et al. performed several works to determine the module $Z_{m} T$ [37-40]. In 2013, this group also reported an expression for the spreading-constriction impedance, a phenomenon that occurs in TE devices since the area of all the TE legs in a module is lower than the area of its ceramic layers [41]. However, it was in 2014 when the ideal models for the characterization of single TE legs and the usual configuration of TE modules were derived [42]. The equivalent circuit of the ideal models are formed by simple elements with different physical meanings (see Table 2.1).

A resistor is represented in the complex plane by a single point in the real axis since its magnitude is not affected by frequency [see Fig. 2.11(a)] and, for TE applications, they are linked to ohmic contributions (e.g. TE materials resistance, metallic strips resistance, electrical contact resistances, wires resistance, etc.). A capacitor is represented in the Nyquist plot by a vertical line that has its origin at the origin of the complex plane, for high frequencies, and decreases its 
imaginary part as the frequency decreases [see Fig. 2.11(b)]. For TE applications, capacitors are linked to the accumulation/release of heat that produces a temperature change.

Table 2.1. Name, impedance function, and reference to the representation in the complex plane of the most common equivalent circuit elements for thermoelectric applications.

\begin{tabular}{lll}
\hline Element name & Impedance function & Reference \\
\hline Resistor $\left(R_{3}\right)$ & $Z_{R}=R_{3}$ & Fig. 2.11(a) \\
Capacitor $\left(C_{2}\right)$ & $Z_{C}=\frac{1}{j \omega C_{2}}$ & Fig. 2.11(b) \\
Constant-temperature Warburg & $Z_{W C T}=R_{4}\left(\frac{j \omega}{\omega_{c r 1}}\right)^{-0.5} \tanh \left[\left(\frac{j \omega}{\omega_{c r 1}}\right)^{0.5}\right]$ & Fig. 2.11(c) \\
Adiabatic Warburg & $Z_{W a}=R_{5}\left(\frac{j \omega}{\omega_{c r 2}}\right)^{-0.5} \operatorname{coth}\left[\left(\frac{j \omega}{\omega_{c r 2}}\right)^{0.5}\right]$ & Fig. 2.11(d) \\
\hline
\end{tabular}

A constant-temperature Warburg element starts at the origin of the complex plane for high frequencies and increases the real part while decreases the imaginary part at the same rate (creating a $45^{\circ}$ slope) until a frequency given by the characteristic angular frequency $\left(f=\omega_{c r l}\right)$ is reached. Once this point is reached, the imaginary part does not decrease as fast as the real part increases, producing a curvature of the spectrum that leads to a minimum in the imaginary part before starting to increase again until reaching a resistor behavior (zero phase angle) [see Fig. 2.11(c)]. In TE applications, as it will be discussed later, constant temperature Warburg elements are linked to the presence of planes where the temperature does not change with frequency (e.g. planes where heat is completely removed, or perfect heat sink conditions). An adiabatic Warburg element, similar to the constant-temperature Warburg element, commences at the origin of the complex plane with a $45^{\circ}$ slope until the frequency $f=\omega_{c r}$, however, lower frequencies than this point lead to a lower imaginary part, creating a vertical line similar to a capacitive response [see Fig. 2.11(d)]. In TE applications, as it will be discussed later, adiabatic Warburg elements are linked to the presence of planes where heat cannot cross. 

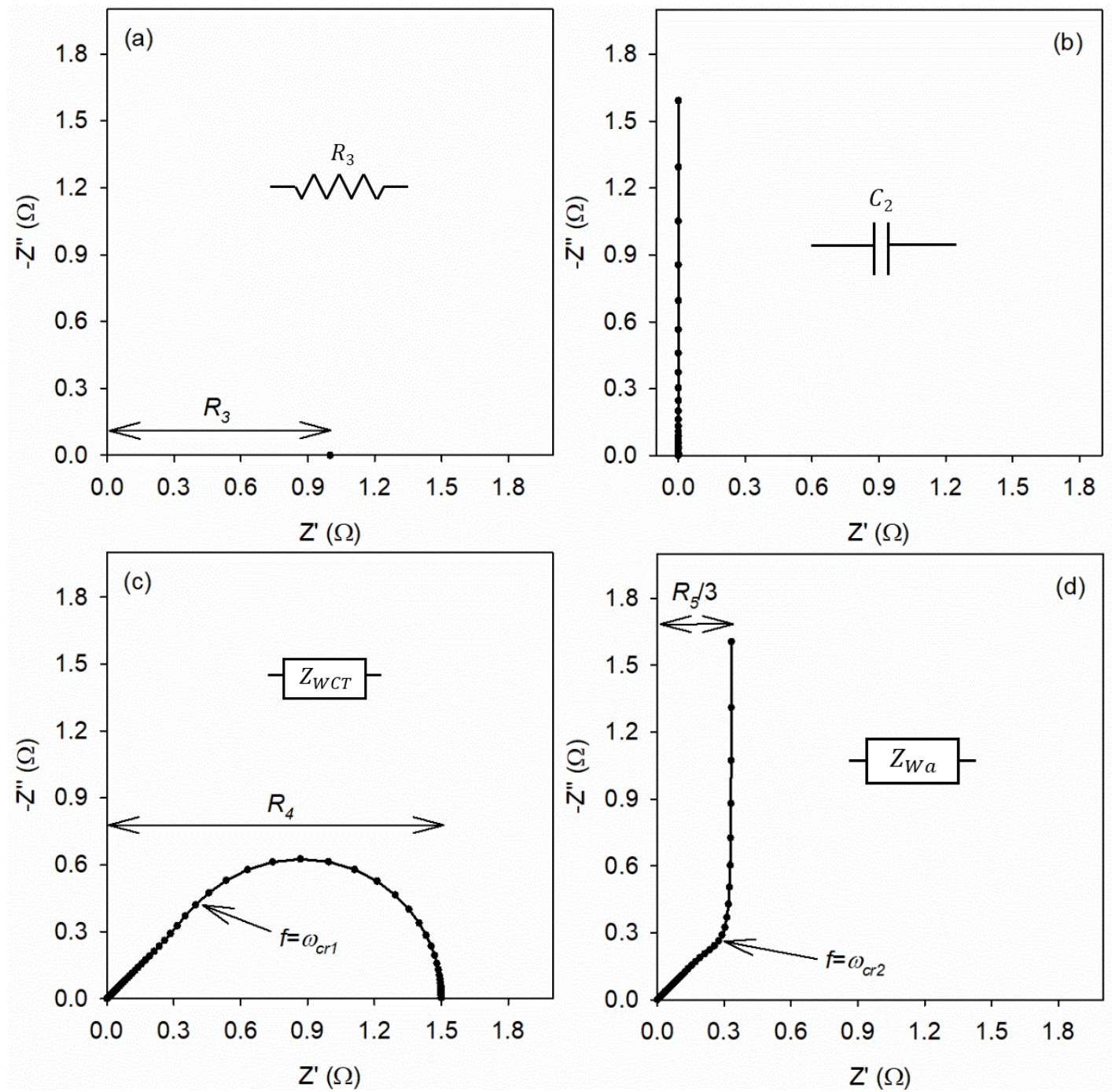

Fig. 2.11. Impedance spectroscopy simulations represented in Nyquist plots of the most common equivalent circuit elements for thermoelectric applications [resistor (a), capacitor (b), constant-temperature Warburg (c), and adiabatic Warburg (d)]. The insets show the symbols of the elements and the arrows indicate the characteristic parameters of each impedance element. The simulations were made using $R_{3}=1 \Omega, C_{l}=100$ $\mathrm{F}, R_{4}=1.5 \Omega, \omega_{c r l}=1 \mathrm{rad} / \mathrm{s}, R_{5}=1 \Omega$, and $\omega_{c r 2}=0.01 \mathrm{rad} / \mathrm{s}$ and the equations presented in Table 2.1 .

To obtain the simplest equivalent circuits that capture the main events occurring in TE systems (ideal equivalent circuits) it is important to understand the TE phenomena in these systems when current/voltage signals are applied. For its obtention, a TE element of length $L$ and cross-sectional area $A$ inserted between two ceramic contacts of length $L_{C}$ with the same cross-sectional area was considered to be in adiabatic condition with the ambient (no convection and no radiation, see Fig. 2.12) [42]. Joule effect was neglected and TE properties independent on temperature were 
assumed, since a very small ac perturbation is applied during an impedance measurement. Two different cases were solved, (i) ideal model for TE legs, assuming a negligible influence of the ceramic contact $\left(L_{C} \rightarrow 0\right)$, and (ii) ideal model for TE modules, where the influence of the contacts cannot be neglected.

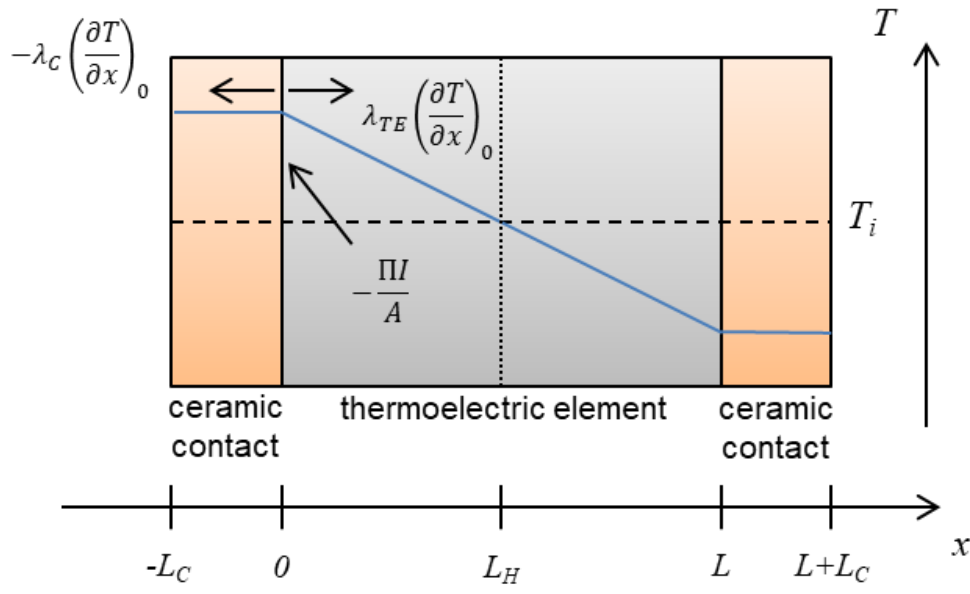

Fig. 2.12. Schematic of a thermoelectric element contacted by two ceramic contacts. The arrows indicate the direction of the heat fluxes appearing at the junctions because of the Peltier effect when an n-type TE material and a positive current are considered. For the Peltier heat, the arrow point to the junction to indicate that electrons dissipate heat to the lattice. The blue solid line, the vertical dotted line, and the horizontal dashed line shows the temperature profile at steady state, the plane where the temperature remains constant, and the initial temperature profile, respectively. This figure was reprinted and modified with permission [42].

The impedance function of a single TE leg $(Z=V / I)$ is given by,

$$
Z=R_{\Omega}+\frac{S[T(L)-T(0)]}{I}=R_{\Omega}-\frac{2 S\left[T(0)-T_{i}\right]}{I},
$$

where $T(0)$ and $T(L)$ are the temperatures at $x=0$ and $x=L$, respectively, and $T_{i}$ is the initial temperature, which is constant along the whole TE leg.

Eq. (2.24) shows the need to determine the temperature at $x=0$ to obtain the impedance function, hence, the heat equation in the frequency domain must be solved,

$$
\frac{\partial^{2} \theta}{\partial x^{2}}=\frac{j \omega}{\alpha_{i}} \theta
$$

where $\alpha_{i}$ represents the thermal diffusivity of each layer ( $i=T E$ for the TE leg and $i=C$ for the ceramic layers), and $\theta$ is the Laplace transform of the temperature with respect to the initial temperature $\left(\theta=\mathscr{L}\left[T-T_{i}\right]\right)$. 
The solution of Eq. (2.25) is given by [42],

$$
\theta=C_{1, i} \sinh \left[\frac{x}{L_{i}}\left(\frac{j \omega}{\omega_{i}}\right)^{0.5}\right]+C_{2, i} \cosh \left[\frac{x}{L_{i}}\left(\frac{j \omega}{\omega_{i}}\right)^{0.5}\right]
$$

and its derivative,

$$
\frac{\partial \theta}{\partial x}=\frac{1}{L_{i}}\left(\frac{j \omega}{\omega_{i}}\right)^{0.5}\left\{C_{1, i} \cosh \left[\frac{x}{L_{i}}\left(\frac{j \omega}{\omega_{i}}\right)^{0.5}\right]+C_{2, i} \sinh \left[\frac{x}{L_{i}}\left(\frac{j \omega}{\omega_{i}}\right)^{0.5}\right]\right\},
$$

where $L_{i}$ is the half the length of the thermoelement $(i=T E)$ or the thickness of the ceramic contact $(i=C), \omega_{i}$ is the characteristic angular frequency of the thermal diffusion for each layer (defined as $\left.\omega_{i}=\alpha_{i} / L_{i}^{2}\right)$, and $C_{l, i}$ and $C_{2, i}$ are constants.

Introducing the boundary conditions for each case [(i) ideal model for TE legs, and (ii) ideal model for TE modules] into Eq. (2.26) and Eq. (2.27), the constants can be determined. The boundary conditions for (i) are,

$$
\begin{aligned}
& \theta(L / 2)=0, \text { at } x=L / 2, \\
& -\frac{S T_{i} i_{0}}{A}+\lambda_{T E}\left(\frac{\partial \theta}{\partial x}\right)_{0}=0, \text { at } x=0,
\end{aligned}
$$

being $i_{0}$ the Laplace transform of the current at $\left.x=0\left(\mathscr{L} I_{0}\right]=i_{0}\right)$ and $\lambda_{T E}$ the thermal conductivity of the thermoelement.

On the other hand, the boundary conditions for (ii) are, Eq. (2.28),

$$
\begin{aligned}
& -\frac{S T_{i} i_{0}}{A}-\lambda_{C}\left(\frac{\partial \theta}{\partial x}\right)_{0, C}+\lambda_{T E}\left(\frac{\partial \theta}{\partial x}\right)_{0, T E}=0, \text { at } x=0, \\
& \left(\frac{\partial \theta}{\partial x}\right)_{-L_{C}}=0, \text { at } x=-L_{C}, \\
& \theta(0)_{T E}=\theta(0)_{C}, \text { at } x=0,
\end{aligned}
$$

being $\lambda_{C}$ the thermal conductivity of the ceramic layers.

After obtaining the constants, the impedance function was calculated and it was found that the response for a single TE element [see Fig. 2.13(a)] is the series combination of a resistor, $R_{\Omega}$, which is the total ohmic contribution (including the intrinsic electric resistance of the TE 
materials, the metallic contacts, and the electrical contact resistances) and a constant-temperature Warburg element, defined as,

$$
Z_{W C T}=\frac{S^{2} T_{i} L}{\lambda_{T E} A}\left(\frac{j \omega}{\omega_{T E}}\right)^{-0.5} \tanh \left[\left(\frac{j \omega}{\omega_{T E}}\right)^{0.5}\right]
$$

Then, for high frequencies, only the ohmic contribution is observed, since there is not enough time for the heat released/absorbed at the junctions to establish a significant temperature difference between both sides of the TE elements. As soon as the frequency starts to decrease, the diffusion of the heat generated at the junctions due to the Peltier effect flows towards the middle of the TE leg, appearing in the impedance spectra as a $45^{\circ}$ slope that indicates a semi-infinite diffusion [42], until the heat coming from one side is removed by the cooling coming from the other side at $L / 2$, blocking the further diffusion of the heat. The closing of the semicircle-like feature is explained since the temperature difference between both sides cannot continue increasing even if the frequency continues decreasing. In the dc limit case (when $\omega \rightarrow 0$ ), a linear temperature profile between both sides is established since the same amount of heat generated at one side is absorbed at the other side.

For the ideal model to characterize TE modules, the influence of the two ceramic contacts has to be added, which leads to a new element in the equivalent circuit that takes the shape of an adiabatic Warburg [see Fig. 2.13(b)],

$$
Z_{W a}=\frac{2 S^{2} T_{i} L_{C}}{\lambda_{C} A}\left(\frac{j \omega}{\omega_{C}}\right)^{-0.5} \operatorname{coth}\left[\left(\frac{j \omega}{\omega_{C}}\right)^{0.5}\right]
$$

At high frequencies, this element also shows a diffusion part in the impedance spectra $\left(45^{\circ}\right.$ slope), which is due to the (semi-infinite) heat diffusion from the junctions towards the outer surfaces of the ceramic contacts [42]. Lower frequencies are governed by the heat accumulation in the ceramics, since heat cannot flow out of the contact material due to the adiabatic conditions, and the response is like a capacitor (vertical line). However, this is only the response of the contacts, which in practice are in parallel with the TE leg response. The total equivalent circuit is the series combination of the resistor $\left(R_{\Omega}\right.$, which represents the total ohmic contribution) with the parallel combination of the constant temperature Warburg $\left(Z_{W C T}\right.$, due to the TE leg) and the adiabatic Warburg ( $Z_{W a}$, due to the contacts) elements. This equivalent circuit can be seen in Fig. 2.13(c). 
As can be seen in Fig. 2.13(c) [and its magnification, Fig. 2.13(d)], the response of the contacts can be seen at high frequencies, whereas at low frequencies the response tends towards the single TE leg response and the influence of the contacts vanishes. This response is common in commercial TE modules because the thermal conductivity and thermal diffusivity of the external ceramic layers are higher than those of the TE legs and the length of the contacts is usually smaller than the TE legs.
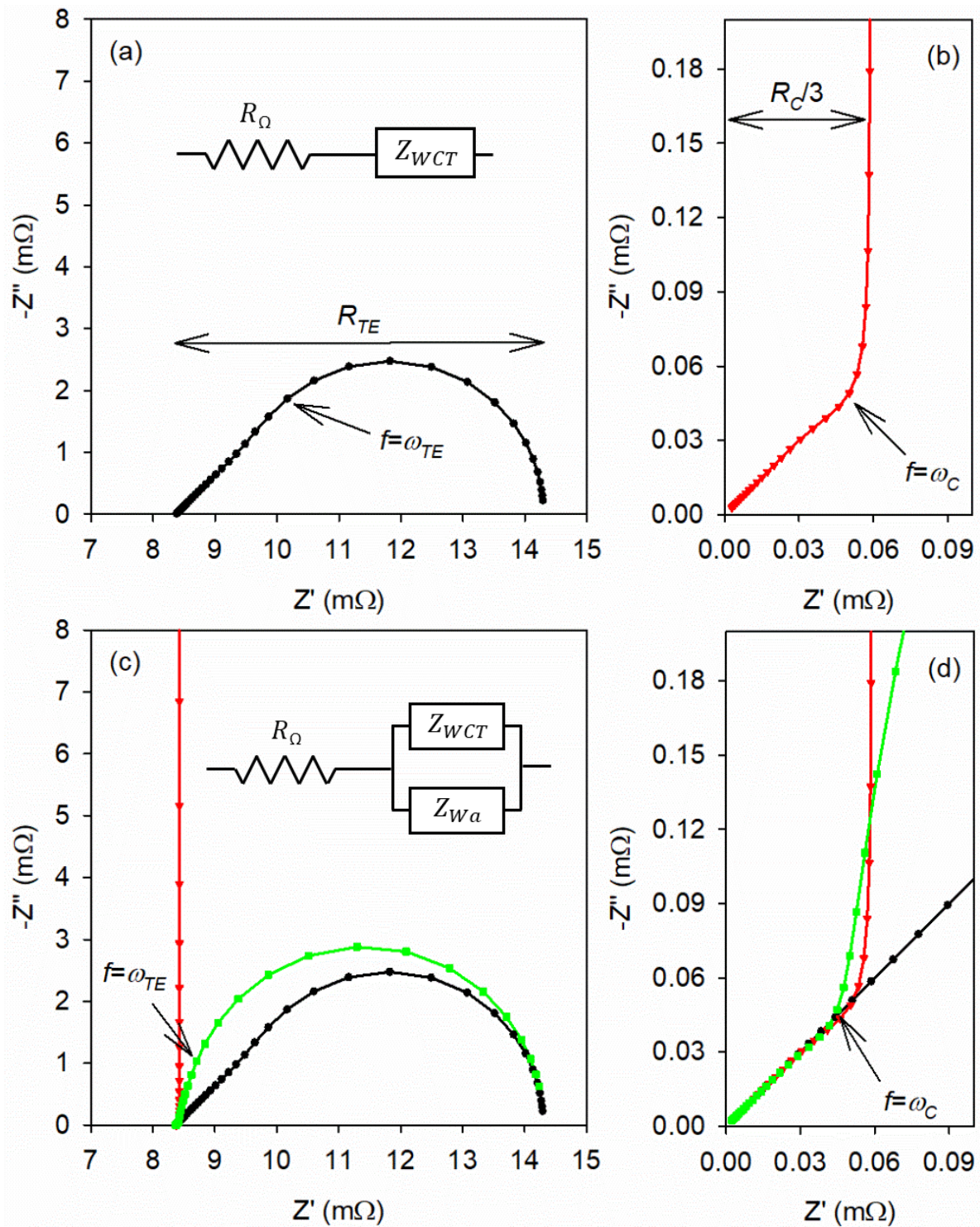

Fig. 2.13. Simulated impedance spectra for a Bi-Te thermoelectric element with $S=190 \mu \mathrm{V} / \mathrm{K}, A=1.96 \mathrm{~mm}^{2}$, $L=1.64 \mathrm{~mm}, \alpha_{T E}=0.37 \mathrm{~mm}^{2} / \mathrm{s}$, and $\lambda_{T E}=1.5 \mathrm{~W} / \mathrm{Km}$ between two ceramic contacts of $L_{C}=0.57 \mathrm{~mm}$, 
$\alpha_{C}=10 \mathrm{~mm}^{2} / \mathrm{s}$, and $\lambda_{C}=35 \mathrm{~W} / \mathrm{Km}$ at $T i=294.7 \mathrm{~K}$. The total ohmic contribution $\left(R_{\Omega}=8.4 \mathrm{~m} \Omega\right)$, was approximated to the ohmic resistance of a single leg considering a resistivity $\rho_{T E}=0.01 \Omega \mathrm{mm}$. (a) Without the influence of the ceramic contacts and using the equivalent circuit in the inset. (b) Under contact influence and assuming that the Peltier flux is only conducted towards the ceramics $\left(\lambda_{T E}=0\right)$. The value of the ohmic resistance $R_{\Omega}$ has been subtracted in the real axis to gain clarity. (c) With contact influence using the equivalent circuit of the inset. The black line represents the same plot of (a) and the red line represents the same plot of (b) with the presence of $R_{\Omega}$. (d) The magnification of the high frequency region in plot (c) where $R_{\Omega}$ has also been subtracted in the real axis.

The validity of both models (single TE leg and TE leg with contacts) were demonstrated with the characterization of a single $\mathrm{Bi}_{2} \mathrm{Te}_{3} \mathrm{TE}$ leg (with and without ceramic contacts) and a commercial TE module [42].

The grey dots in Fig. 2.14(a) show the experimental measurement obtained with a single TE leg with a negligible layer of $\mathrm{Ag}$ paint to make the contacts, which clearly exhibits the expected response [Fig. 2.13(a)]. The blue line in Fig. 2.14(a) represents the fitting performed with the equivalent circuit in the inset of Fig. 2.13(a), which provided $R_{\Omega}=84 \mathrm{~m} \Omega, R_{T E}=5.8 \mathrm{~m} \Omega$, and $\omega_{T E}=2.0 \mathrm{rad} / \mathrm{s}$. Fig. 2.14(b) shows the IS measurement obtained with a TE leg sandwiched between two pieces of the usual copper/ceramic layer found in commercial TE devices. The influence of the copper layer is neglected due to its high thermal conductivity and small thickness. As expected, the response is very similar to the green line in Fig. 2.13(c).
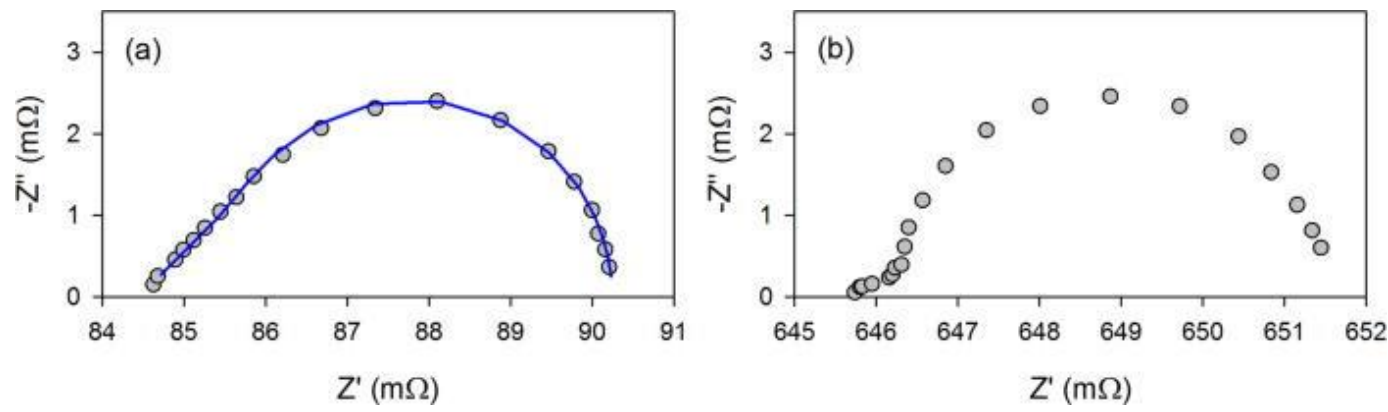

Fig. 2.14. Impedances of p-type thermoelements (a) with very thin $\mathrm{Ag}$ contacts and (b) with $\mathrm{Cu} / \mathrm{ceramic}$ contacts. Line in (a) represents the fitting with the equivalent circuit in the inset of Fig. 2.13(a). The fitting provided $R_{\Omega}=84 \mathrm{~m} \Omega, R_{T E}=5.8 \mathrm{~m} \Omega$, and $\omega_{T E}=2.0 \mathrm{rad} / \mathrm{s}$. This figure was reprinted with permission [42].

Finally, Fig. 2.15 also shows the response of a commercial TE module (grey dots) and the fitting with the equivalent circuit shown in the inset of Fig. 2.13(c) (blue line). It should be noticed that the response of a TE module is multiplied by the number of legs of the TE module since they are connected in series. The commercial TE module also showed the expected response [Fig. 
$2.13(\mathrm{c})]$ and the fitting provided $R_{\Omega}=4.29 \Omega, R_{C}=0.149 \Omega, \omega_{C}=6.08 \mathrm{rad} / \mathrm{s}, R_{T E}=2.585 \Omega$, and $\omega_{T E}=0.24 \mathrm{rad} / \mathrm{s}$.

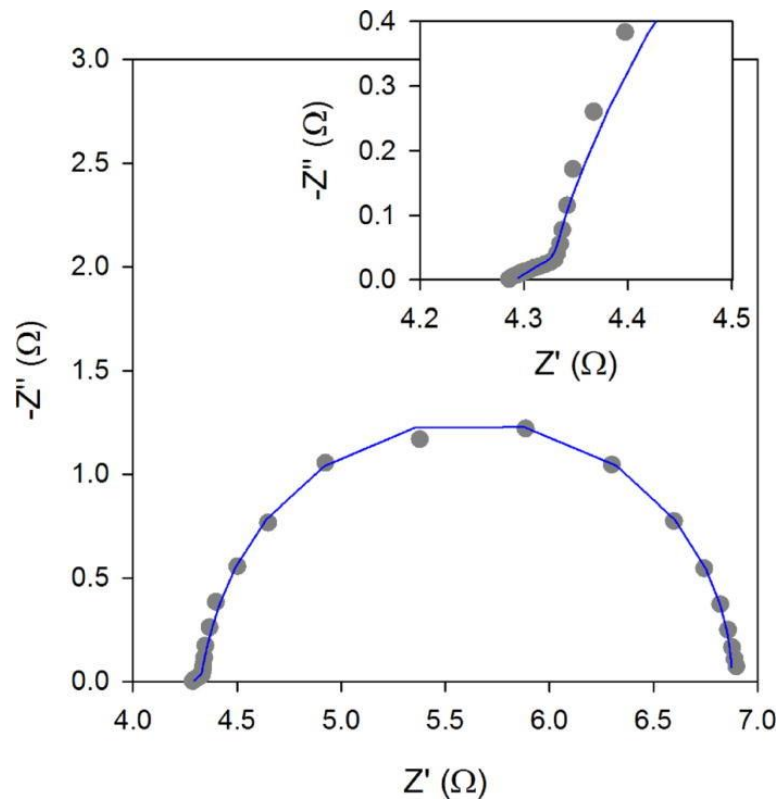

Fig. 2.15. Nyquist plot of a 254-leg thermoelectric module suspended in air. The inset shows a magnified high-frequency part. The line corresponds to the fitting with the equivalent circuit in the inset of Fig. 2.13(c). The fitting provided $R_{\Omega}=4.29 \Omega, R_{C}=0.149 \Omega, \omega_{C}=6.08 \mathrm{rad} / \mathrm{s}, R_{T E}=2.585 \Omega$, and $\omega_{T E}=0.24 \mathrm{rad} / \mathrm{s}$. This figure was reprinted with permission [42].

\section{References}

[1] T.J. Seebeck, Magnetische Polarisation der Metalle und Erze durch Temperatur-Differenz, Abh. Akad. Wiss. Berlin 1820-21. (1822) 289-346. https://archive.org/details/magnetischepolar00seebuoft/page/1/mode/2up (accessed February 20, 2020).

[2] ASTM Committee E20 on Temperature Measurement, Manual on the Use of Thermocouples in Temperature Measurement (ASTM Manual Series), ASTM International, 2003.

[3] J.C. Peltier, Nouvelles expériences sur la caloricité des courants électrique, Ann. Chim. Phys. 56 (1834) 371-386.

[4] A.F. Ioffe, L.S. Stil'bans, E.K. Iordanishvili, T.S. Stavitskaya, A. Gelbtuch, 
Semiconductor Thermoelements and Thermoelectric Cooling, Phys. Today. 12 (1959) 4242. https://doi.org/10.1063/1.3060810.

[5] W. Thomson, On a Mechanical Theory of Thermo-Electric Currents, Proc. R. Soc. Edinburgh. 3 (1857) 91-98. https://doi.org/10.1017/s0370164600027310.

[6] E. Altenkirch, On the effectiveness of the thermopile, Phys. Zeitschrift. 10 (1909) 560568.

[7] E. Altenkirch, Electrothermal cooling and reversible electric heating, Phys. Zeitschrift. 12 (1911) 920-924.

[8] G. Chen, M.S. Dresselhaus, G. Dresselhaus, J.P. Fleurial, T. Caillat, Recent developments in thermoelectric materials, Int. Mater. Rev. 48 (2013) 45-66. https://doi.org/10.1179/095066003225010182.

[9] M. V. Vedernikov, E.K. Iordanishvili, A.F.Ioffe and origin of modern semiconductor thermoelectric energy conversion, in: Int. Conf. Thermoelectr. ICT, Proc., IEEE, 1998: pp. 37-42. https://doi.org/10.1109/ict.1998.740313.

[10] D. Beretta, N. Neophytou, J.M. Hodges, M.G. Kanatzidis, D. Narducci, M. MartinGonzalez, M. Beekman, B. Balke, G. Cerretti, W. Tremel, A. Zevalkink, A.I. Hofmann, C. Müller, B. Dörling, M. Campoy-Quiles, M. Caironi, Thermoelectrics: From history, a window to the future, Mater. Sci. Eng. R Reports. 138 (2019) 100501. https://doi.org/10.1016/j.mser.2018.09.001.

[11] B. Poudel, Q. Hao, Y. Ma, Y. Lan, A. Minnich, B. Yu, X. Yan, D. Wang, A. Muto, D. Vashaee, X. Chen, J. Liu, M.S. Dresselhaus, G. Chen, Z. Ren, High-thermoelectric performance of nanostructured bismuth antimony telluride bulk alloys, Science (80-. ). 320 (2008) 634-638. https://doi.org/10.1126/science.1156446.

[12] L. Hu, H. Wu, T. Zhu, C. Fu, J. He, P. Ying, X. Zhao, Tuning Multiscale Microstructures to Enhance Thermoelectric Performance of n-Type Bismuth-Telluride-Based Solid Solutions, Adv. Energy $\quad$ Mater. 5 (2015) 1500411. https://doi.org/10.1002/aenm.201500411.

[13] A. Nozariasbmarz, F. Suarez, J.H. Dycus, M.J. Cabral, J.M. LeBeau, M.C. Öztürk, D. 
Vashaee, Thermoelectric generators for wearable body heat harvesting: Material and device concurrent optimization, Nano Energy. $67 \quad$ (2020) 104265. https://doi.org/10.1016/j.nanoen.2019.104265.

[14] S. Il Kim, K.H. Lee, H.A. Mun, H.S. Kim, S.W. Hwang, J.W. Roh, D.J. Yang, W.H. Shin, X.S. Li, Y.H. Lee, G.J. Snyder, S.W. Kim, Dense dislocation arrays embedded in grain boundaries for high-performance bulk thermoelectrics, Science (80-. ). 348 (2015) 109114. https://doi.org/10.1126/science.aaa4166.

[15] G. Rogl, P. Rogl, Skutterudites, a most promising group of thermoelectric materials, Curr. Opin. Green Sustain. Chem. 4 (2017) 50-57. https://doi.org/10.1016/j.cogsc.2017.02.006.

[16] J. Yu, C. Fu, Y. Liu, K. Xia, U. Aydemir, T.C. Chasapis, G.J. Snyder, X. Zhao, T. Zhu, Unique Role of Refractory Ta Alloying in Enhancing the Figure of Merit of $\mathrm{NbFeSb}$ Thermoelectric Materials, Adv. Energy Mater. $8 \quad$ (2018) 1701313. https://doi.org/10.1002/aenm.201701313.

[17] E. Rausch, B. Balke, J.M. Stahlhofen, S. Ouardi, U. Burkhardt, C. Felser, Fine tuning of thermoelectric performance in phase-separated half-Heusler compounds, J. Mater. Chem. C. 3 (2015) 10409-10414. https://doi.org/10.1039/c5tc01196e.

[18] M. Schwall, B. Balke, Phase separation as a key to a thermoelectric high efficiency, Phys. Chem. Chem. Phys. 15 (2013) 1868-1872. https://doi.org/10.1039/c2cp43946h.

[19] L.-D. Zhao, S.-H. Lo, Y. Zhang, H. Sun, G. Tan, C. Uher, C. Wolverton, V.P. Dravid, M.G. Kanatzidis, Ultralow thermal conductivity and high thermoelectric figure of merit in SnSe crystals, Nature. 508 (2014) 373-377. https://doi.org/10.1038/nature13184.

[20] M.R. Burton, T. Liu, J. McGettrick, S. Mehraban, J. Baker, A. Pockett, T. Watson, O. Fenwick, M.J. Carnie, Thin Film Tin Selenide (SnSe) Thermoelectric Generators Exhibiting Ultralow Thermal Conductivity, Adv. Mater. 30 (2018) 1801357. https://doi.org/10.1002/adma.201801357.

[21] C. Chang, M. Wu, D. He, Y. Pei, C.F. Wu, X. Wu, H. Yu, F. Zhu, K. Wang, Y. Chen, L. Huang, J.F. Li, J. He, L.D. Zhao, 3D charge and 2D phonon transports leading to high outof-plane ZT in n-type SnSe crystals, Science (80-. ). 360 (2018) 778-783. 
https://doi.org/10.1126/science.aaq1479.

[22] R. Chetty, A. Bali, R.C. Mallik, Tetrahedrites as thermoelectric materials: An overview, J. Mater. Chem. C. 3 (2015) 12364-12378. https://doi.org/10.1039/c5tc02537k.

[23] J. Heo, G. Laurita, S. Muir, M.A. Subramanian, D.A. Keszler, Enhanced thermoelectric performance of synthetic tetrahedrites, Chem. Mater. 26 (2014) 2047-2051. https://doi.org/10.1021/cm404026k.

[24] X. Lu, D.T. Morelli, Y. Xia, V. Ozolins, Increasing the thermoelectric figure of merit of tetrahedrites by Co-doping with nickel and zinc, Chem. Mater. 27 (2015) 408-413. https://doi.org/10.1021/cm502570b.

[25] L.L. Baranowski, G. Jeffrey Snyder, E.S. Toberer, Effective thermal conductivity in thermoelectric materials, J. Appl. Phys. $113 \quad$ (2013) 204904. https://doi.org/10.1063/1.4807314.

[26] D.K. Aswal, R. Basu, A. Singh, Key issues in development of thermoelectric power generators: High figure-of-merit materials and their highly conducting interfaces with metallic interconnects, Energy Convers. Manag. $114 \quad$ (2016) 50-67. https://doi.org/10.1016/j.enconman.2016.01.065.

[27] Z.G. Chen, G. Hana, L. Yanga, L. Cheng, J. Zou, Nanostructured thermoelectric materials: Current research and future challenge, Prog. Nat. Sci. Mater. Int. 22 (2012) 535-549. https://doi.org/10.1016/j.pnsc.2012.11.011.

[28] H.S. Kim, W. Liu, Z. Ren, The bridge between the materials and devices of thermoelectric power generators, Energy Environ. Sci. $10 \quad$ (2017) 69-85. https://doi.org/10.1039/c6ee02488b.

[29] K. Teffah, Y. Zhang, X. Mou, Modeling and Experimentation of New Thermoelectric Cooler-Thermoelectric Generator Module, Energies. $11 \quad$ (2018) 576. https://doi.org/10.3390/en11030576.

[30] X. Zhang, L.D. Zhao, Thermoelectric materials: Energy conversion between heat and electricity, J. Mater. 1 (2015) 92-105. https://doi.org/10.1016/j.jmat.2015.01.001.

[31] R. M. Atta, Thermoelectric Cooling, in: P. Arangures (Ed.), IntechOpen, 2018. 
https://doi.org/10.5772/intechopen.75791.

[32] E. Barsoukov, J.R. (James R. Macdonald, Impedance spectroscopy : theory, experiment, and applications., 3rd ed., 2018.

[33] S. Dilhaire, L.D. Patino-Lopez, S. Grauby, J.M. Rampnoux, S. Jorez, W. Claeys, Determination of ZT of PN thermoelectric couples by AC electrical measurement, in: Int. Conf. Thermoelectr. ICT, Proc., Institute of Electrical and Electronics Engineers Inc., 2002: pp. 321-324. https://doi.org/10.1109/ICT.2002.1190330.

[34] A.D. Downey, E. Timm, P.F.P. Poudeu, M.G. Kanatzidis, H. Shock, T.P. Hogan, Application of transmission line theory for modeling of a thermoelectric module in multiple configurations for AC electrical measurements, in: Mater. Res. Soc. Symp. Proc., Cambridge University Press, 2006: pp. 425-430. https://doi.org/10.1557/proc-0886-f1007.

[35] A.D. Downey, T.P. Hogan, B. Cook, Characterization of thermoelectric elements and devices by impedance spectroscopy, Rev. Sci. Instrum. 78 (2007) 093904. https://doi.org/10.1063/1.2775432.

[36] A.D. Downey, T.P. Hogan, Proceedings of the 24th International Conference on Thermoelectrics, Clemson, SC. (2005) 79-82.

[37] A. De Marchi, V. Giaretto, The elusive half-pole in the frequency domain transfer function of Peltier thermoelectric devices, Rev. Sci. Instrum. 82 (2011) 034901. https://doi.org/10.1063/1.3558696.

[38] A. De Marchi, V. Giaretto, An accurate new method to measure the dimensionless figure of merit of thermoelectric devices based on the complex impedance porcupine diagram, Rev. Sci. Instrum. 82 (2011). https://doi.org/10.1063/1.3656074.

[39] A. De Marchi, V. Giaretto, S. Caron, A. Tona, A. De Marchi, V. Giaretto, S. Caron, A. Tona, A Novel zT Meter Based on the Porcupine Method and a Survey on the Size of the Snout Correction Needed for Various Thermoelectric Devices, J. Electron. Mater. 42 (2013) 2067-2072. https://doi.org/10.1007/s11664-013-2530-2.

[40] A. De Marchi, V. Giaretto, The Peltier driven frequency domain approach in thermal 


\section{Chapter 2}

analysis, Rev. Sci. Instrum. 85 (2014). https://doi.org/10.1063/1.4897189.

[41] F. Casalegno, A. De Marchi, V. Giaretto, Frequency domain analysis of spreadingconstriction thermal impedance, Rev. Sci. Instrum. $84 \quad$ (2013) 024901. https://doi.org/10.1063/1.4789765.

[42] J. García-cañadas, G. Min, Impedance spectroscopy models for the complete characterization of thermoelectric materials, J. Appl. Phys. 116 (2014). https://doi.org/10.1063/1.4901213. 
3. Thermal conductivity, electrical resistivity, and dimensionless figure of merit $(z T)$ determination of thermoelectric materials by impedance spectroscopy up to $250{ }^{\circ} \mathrm{C}$ 


\section{Thermal conductivity, electrical resistivity, and dimensionless figure of merit $(z T)$ determination of thermoelectric materials by impedance spectroscopy up to $250{ }^{\circ} \mathrm{C}$}

Braulio Beltrán-Pitarch ${ }^{1}$, Jesús Prado-Gonjal ${ }^{2}$, Anthony V. Powell ${ }^{2}$, Pawel Ziolkowski ${ }^{3}$, Jorge García-Cañadas ${ }^{1, *}$

${ }^{1}$ Department of Industrial Systems Engineering and Design, Universitat Jaume I, Campus del Riu Sec, 12071 Castellón, Spain

${ }^{2}$ Department of Chemistry, University of Reading, RG6 6AD, Reading, UK

${ }^{3}$ Institute of Materials Research, German Aerospace Center, Linder Höhe, 51147 Köln, Germany *e-mail: garciaj@uji.es 


\begin{abstract}
Impedance spectroscopy (IS) has been shown as a promising method to characterize thermoelectric (TE) materials and devices. In particular, the possibility to determine the thermal conductivity $\lambda$, electrical conductivity $\sigma$, and the dimensionless figure of merit $z T$ of a TE element, if the Seebeck coefficient $S$ is known, has been reported; although so far for a high-performance TE material $\left(\mathrm{Bi}_{2} \mathrm{Te}_{3}\right)$ at room temperature. Here, we demonstrate the capability of this approach at temperatures up to $250^{\circ} \mathrm{C}$ and for a material with modest TE properties. Moreover, we compare the results obtained with values from commercial equipment and quantify the precision and accuracy of the method. This is achieved by measuring the impedance response of a skutterudite material contacted by $\mathrm{Cu}$ contacts. The method shows excellent precision (random errors $<4.5 \%$ for all properties) and very good agreement with the results from commercial equipment $(<4 \%$ for $\lambda$, between $4 \%$ and $6 \%$ for $\sigma$, and $<8 \%$ for $z T$ ), which proves its suitability to accurately characterize bulk TE materials. Especially, the capability to provide $\lambda$ with good accuracy represents a useful alternative to the laser flash method, which typically exhibits higher errors and requires the measurement of additional properties (density and specific heat), which are not necessarily needed to obtain the $z T$.
\end{abstract}




\section{Graphical abstract}

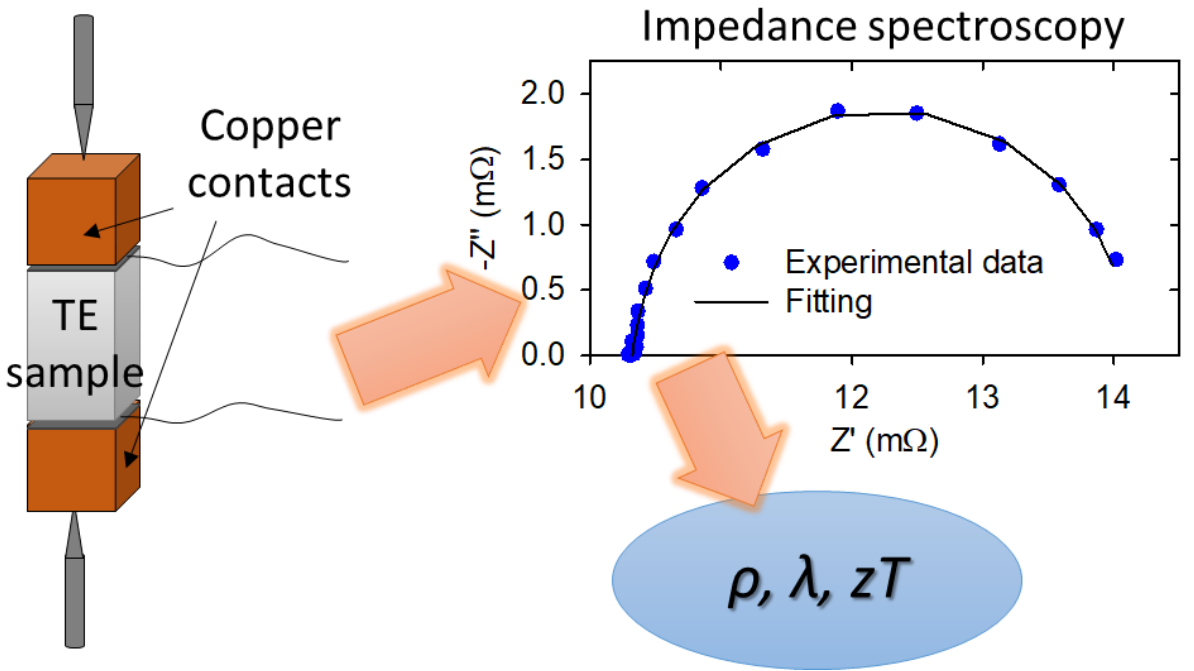

Fig. 3.1. Graphical abstract of the work: Thermal conductivity, electrical resistivity, and dimensionless figure of merit $(z T)$ determination of thermoelectric materials by impedance spectroscopy up to $250{ }^{\circ} \mathrm{C}$. 


\subsection{Introduction}

An efficient use of energy becomes increasingly important. Thermoelectric (TE) devices are considered among other means to improve the energy efficiency of combustion engines or to harvest energy from industrial processes by a conversion of waste heat to electric power. The efficiency of a TE material is related to the dimensionless figure of merit $z T=\sigma S^{2} T / \lambda$, where $\sigma$ is the electrical conductivity, $S$ is the Seebeck coefficient, $T$ is the absolute temperature, and $\lambda$ is the thermal conductivity. The search for more efficient materials is typically guided by the $z T$ improvement, which can be obtained by adjustment of the composition, doping, microstructural effects and nano-structuring [1]. $z T$ is commonly obtained from the independent determination of the three properties that define it $(\sigma, S$, and $\lambda)$. For this reason, TE characterization is a timeconsuming task which usually requires several apparatuses. Moreover, the determination of the thermal conductivity is especially troublesome, since heat losses are difficult to minimize and high errors are frequently present. The laser flash method [2] is the most frequently used technique for the thermal conductivity determination [3], but it requires the additional measurement of two more properties (density and specific heat), which complicates the TE characterization and introduces measurement uncertainties, especially with respect to the specific heat. Under this scenario, new techniques and methods are highly desired to improve the task of TE characterization by reducing the required efforts, the time, and by improving accuracy.

Impedance spectroscopy (IS) has been shown as a promising method to characterize TE materials and devices [4-8]. This technique has been employed in many fields of research (fuel cells [9], supercapacitors [10], construction [11], corrosion [12], photovoltaics [13], etc.). Due to this, impedance equipment can be easily found in many research institutions, and highly accurate and reliable apparatus exist. In our previous work [6,14], we identified, for a high-performance TE material $\left(\mathrm{Bi}_{2} \mathrm{Te}_{3}\right)$ and at room temperature, the possibility to determine its thermal conductivity, electrical resistivity, and $z T$, if the Seebeck coefficient is known. However, for materials characterization, this approach has neither been extended to high temperatures nor has been evaluated for low-performance TE materials. The latter could be troublesome due to the very small impedance signals typically registered (in the $\mathrm{m} \Omega$ range), which might be close to the equipment limitation [14]. The signal originates from the low Seebeck voltage induced by the Peltier effect when the current is applied. In addition, a quantification of the precision and 
accuracy of the impedance method to determine the TE properties of bulk materials using this approach has not been previously provided.

In this work, we extend the previously mentioned approach above room temperature (up to $250^{\circ} \mathrm{C}$ ) and demonstrate its capability to measure low-performance TE materials. This is achieved using a skutterudite material, which exhibits low $z T(<0.2)$ around room temperature. The sample is measured in a homemade setup which is adapted to perform measurements in a 4-probe mode. Using experimentally measured values of the Seebeck coefficient from a commercial instrument, the rest of TE properties were determined by the impedance method using a suitable equivalent circuit. Finally, the precision and accuracy of the technique was evaluated by a comparison of the obtained TE properties with results from commercial equipment.

\subsection{Experimental setup}

The homemade setup used for the impedance characterization contains a sample holder suitable for TE materials of bar shape, which is shown in Fig. 3.2. To perform the measurements, the TE sample is sandwiched between two pieces of copper of same cross-sectional area as the TE material and with $2 \mathrm{~mm}$ thickness. This is required to ensure a homogeneous electrical current at the junctions and a uniform Peltier effect. A very thin layer of $\mathrm{Ga}_{62} \mathrm{In}_{22} \mathrm{Sn}_{16}$ liquid metal (Ref. 14634, Alfa Aesar) was spread homogeneously at the junctions, which were previously polished and cleaned with acetone to provide a good thermal and electrical contact. For the same reason, it is important that the $\mathrm{Cu}$ and TE material surfaces brought into contact are as flat as possible. Two very thin copper wires (15 $\mu \mathrm{m}$ diameter, Alfa Aesar) were inserted in both junctions for the measurement of the voltage difference across the TE sample (see inset of Fig. 3.2). The very thin diameter minimizes the heat losses by conduction through the wires, and also allows the wires to be inserted at the junctions.

Once assembled, the sample is clamped at the sample holder by two sharpened stainless-steel screws, which act as probes to supply the current flow. These two screws are screwed by nuts at holed ceramics (Macor, Corning) which provide electrical insulation. The stainless-steel screws are connected to thick copper wires insulated by ceramic beads (see Fig. 3.2). Stainless-steel screws were chosen due to their low thermal conductivity $\left(\approx 14 \mathrm{~W} / \mathrm{K}^{-1} \mathrm{~m}^{-1}\right)$, which reduces heat losses by conduction. They were also sharpened for the same purpose. The very thin copper wires 
that measure the potential difference are clamped at the sample holder by two nuts screwed with stainless steel screws, which are held by the ceramic plates (see Fig. 3.2). These screws are also connected to thick copper wires insulated by ceramic beads. The bottom holed ceramic disc is fixed at four threaded studs by nuts, while the top ceramic is free to move to be able to allocate samples of different lengths, and additionally provide certain pressure to the contacts. A stainlesssteel base is also held by nuts at the studs. This base is used to hold a band heater (Ref. MB2E2JN1-B12, Watlow) which surrounds the sample holder and is used to provide different ambient temperatures. The ambient temperature is measured by a K-type thermocouple (RS) placed close to the TE sample (see Fig. 3.2), whose temperature is controlled by a temperature controller (Watlow EZ Zone PM) which powers the heater.

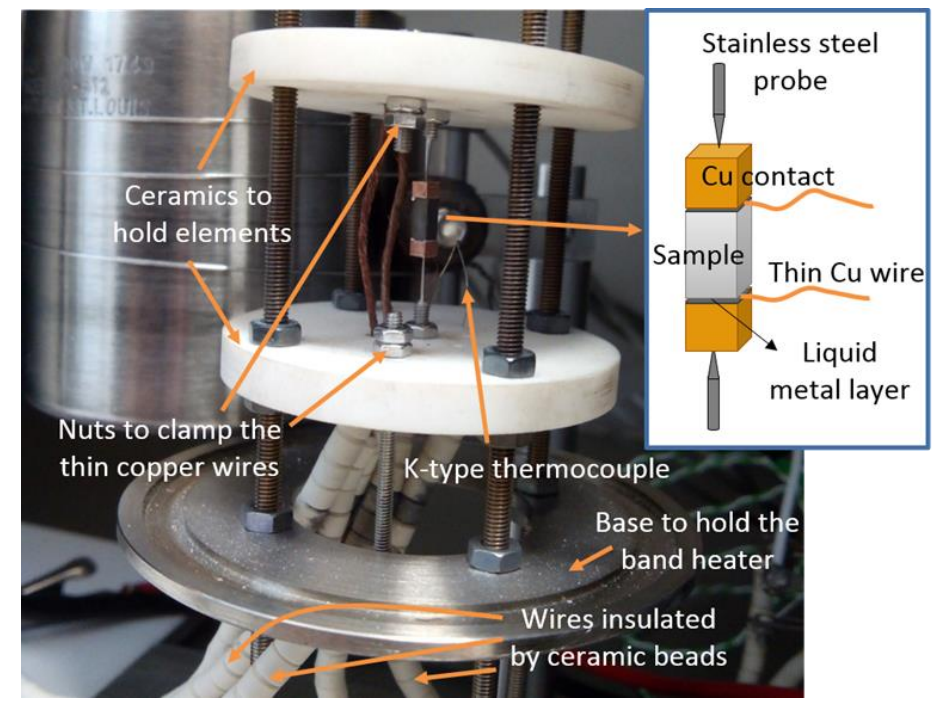

Fig. 3.2. Photograph of the sample holder employed for the impedance characterization of thermoelectric materials. The inset describes schematically how the sample is contacted.

All the impedance measurements were performed inside a stainless-steel vacuum chamber at pressure values $<10^{-4} \mathrm{mbar}$ in order to eliminate convection heat losses. In addition, the metallic vacuum chamber also serves as a Faraday cage, which reduces electromagnetic noise during the measurements. The TE sample used in this study was a tetragonal-shaped and isotropic n-type skutterudite $\left(\mathrm{CoSb}_{2.75} \mathrm{Sn}_{0.05} \mathrm{Te}_{0.20}\right)$, which was cut with a diamond saw of $0.3 \mathrm{~mm}$ diameter from a disc pellet. A suitable cutting is important to obtain a crack free sample of highly uniform crosssectional area. The cross-sectional area of the sample was $2.30 \mathrm{~mm} \times 2.11 \mathrm{~mm}$ and its length was $5.01 \mathrm{~mm}$. The skutterudite sample was characterized employing commercial equipment before 
the impedance measurements. This characterization with commercial equipment was performed to the original disc pellet. A Linseis LSR-3 equipment was used to determine the electrical resistivity and the Seebeck coefficient. For the thermal conductivity, a Netzsch LFA 447 laser flash apparatus was employed. The specific heat of the sample was determined using the same equipment via a comparative method with a Pyroceram reference sample. The density of the sample, which is also required for the determination of the thermal conductivity by the laser flash method, was measured using an Archimedes balance.

A PGSTAT30 potentiostat (Metrohm Autolab B.V.) equipped with a FRA2 impedance module and a BOOSTER10A, which amplifies the maximum current of the equipment up to 10 A, was used to perform the IS measurements. Although such large currents were not reached, the booster is used in order to reduce a systematic jump in the real impedance of $\approx 70 \mu \Omega$ produced due to a change in the gain of the equipment, which occurs at frequencies around $25 \mathrm{~Hz}$ (see Fig. S3.1). This jump can be significantly reduced if measurements are performed in the largest possible current range. At each temperature, the impedance measurement was conducted in 40 logarithmically distributed frequency steps between $5 \mathrm{mHz}$ and $10 \mathrm{kHz}$. An ac current without steady component $\left(I_{d c}=0 \mathrm{~A}\right)$ was employed using a maximum integration time of $2 \mathrm{~s}$ and 2 minimum integration cycles. The ac current amplitude to be used needs to be optimized, since significant differences in the spectra can be observed when this parameter is varied (see Fig. S3.2). This optimization is described in Sec. 3.3.2. Nova 1.11 software was used to control the potentiostat and record the experimental signals. Experimental impedance spectra were fitted to equivalent circuits using Zview software.

\subsection{Results and discussion}

\subsubsection{The equivalent circuit}

In order to extract the properties of interest from the impedance spectra, the experimental results are typically fitted to a suitable theoretical model (equivalent circuit), which should describe the physics of the device. The equivalent circuit corresponding to the case of a TE sample contacted by two metallic contacts has been previously reported [6], and consists of an ohmic resistance $R_{\Omega}$ connected in series with the parallel combination of two Warburg elements: a constant temperature Warburg impedance $Z_{W C T}$, which relates to the properties of the TE sample, 
and an adiabatic Warburg impedance $Z_{W a}$, which is described by $S$ and the properties of the metallic contact material. These elements are defined as follows [6,15]:

$$
\begin{aligned}
& R_{\Omega}=\frac{\rho_{T E} L}{A}, \\
& Z_{W C T}=R_{T E}\left(\frac{j \omega}{\omega_{T E}}\right)^{-0.5} \tanh \left[\left(\frac{j \omega}{\omega_{T E}}\right)^{0.5}\right], \\
& Z_{W a}=R_{C}\left(\frac{j \omega}{\omega_{C}}\right)^{-0.5} \tanh \left[\left(\frac{j \omega}{\omega_{C}}\right)^{0.5}\right],
\end{aligned}
$$

where $\rho_{T E}, L$, and $A$ are the electrical resistivity, length, and cross-sectional area of the TE material, respectively. $R_{T E}$ is the TE resistance given by

$$
R_{T E}=\frac{S^{2} T_{i} L}{\lambda_{T E} A}
$$

where $T_{i}$ is the absolute ambient temperature and $\lambda_{T E}$ is the thermal conductivity of the TE material. $j=(-1)^{0.5}, \omega$ is the angular frequency, and $\omega_{T E}$ is the characteristic angular frequency of thermal diffusion in the TE sample $\left[\omega_{T E}=\alpha_{T E} /(L / 2)^{2} ; \alpha_{T E}\right.$ being the thermal diffusivity of the TE material]. $R_{C}$ is the TE resistance induced by the metallic contact ( $\mathrm{Cu}$ pieces) given by

$$
R_{C}=2 \frac{S^{2} T_{i} L_{C}}{\lambda_{C} A}
$$

where $\lambda_{C}$ and $L_{C}$ are the thermal conductivity and length of the metallic contact material, respectively. Finally, $\omega_{C}$ is the characteristic angular frequency of thermal diffusion in the contact $\left(\omega_{C}=\alpha_{C} / L_{C}{ }^{2} ; \alpha_{C}\right.$ being the thermal diffusivity of the contact).

It should be noted that due to the high thermal conductivity of copper $[\approx 400 \mathrm{~W} /(\mathrm{Km})], R_{C}$ has a very low value $\left(\approx 15 \mu \Omega\right.$ at room temperature). For this reason, the slope-1 part of the $Z_{W a}$ element is not clearly observed experimentally and this element takes the form of a capacitor, with its impedance function described by $Z_{C c}=1 /(j \omega C c)$, being $C c=\left(R_{C} \omega_{C}\right)^{-1}$ [6]. This equivalent circuit, which was used to perform the fittings to the experimental impedance results, is shown in the inset of Fig. 3.3(a). It describes a semicircle in the complex plane (Nyquist plot), where the ohmic resistance and $R_{T E}$ can be clearly identified as the high frequency (left side) intercept with the real axis and the diameter of the semicircle, respectively [6]. 
From the fittings, $R_{\Omega}, R_{T E}, \omega_{T E}$, and $C_{C}$ can be obtained. Hence, using Eqs. (2.1) and (3.4), the electrical resistivity, thermal conductivity (if $S$ is known), and $z T$ of the TE material can be obtained. From Eq. (2.1),

$$
\rho_{T E}=\frac{R_{\Omega} A}{L} .
$$

From Eq. (3.4),

$$
\lambda_{T E}=\frac{S^{2} T_{i} L}{R_{T E} A} .
$$

Combining Eqs. (2.1) and (3.4),

$$
z T=\frac{R_{T E}}{R_{\Omega}} .
$$

\subsubsection{Current amplitude optimization}

Before characterizing the skutterudite sample at the different temperatures, it is important to identify the suitable current amplitude to be used during the impedance measurements. Fig. S3.2 shows impedance spectra performed at an ambient temperature of $50{ }^{\circ} \mathrm{C}$ for different current amplitudes $I_{a c}$. It can be observed that the spectra vary with the current amplitude, probably due to the existence of Joule effect and/or the dependence of the TE properties on temperature. From Fig. S3.2(a), which shows the experiment at the lowest current amplitude (52 mA), it can be observed that the spectrum is somewhat noisy, due to the existence of several points which deviate from the shape of a semicircle. The noise is reduced when $78 \mathrm{~mA}$ amplitude is used, yielding a better correspondence to the semicircle characteristic although some points still deviate in the higher frequency range [see inset of Fig. S3.2 (b)]. At amplitudes of $104 \mathrm{~mA}$ and above, the noise becomes negligible, consequently, this amplitude is considered as optimum, since it provides a sufficient Peltier effect to obtain a clear TE signal in the impedance spectrum while a minimal Joule heat liberation is ensured at the same time. This amplitude corresponds to a Peltier heat power per unit area $\left(S T_{i} I_{a c} / A\right)$ generated at the junctions of $1000 \mathrm{~W} / \mathrm{m}^{2}$. Using this value as reference, the optimum current amplitude to be employed at the different temperatures is calculated by $I_{a c}=\left(1000 \mathrm{~W} / \mathrm{m}^{2}\right) A /\left(S T_{i}\right)$, obtaining values of $84,69,58$, and $49 \mathrm{~mA}$ for the ambient temperatures of $100,150,200$, and $250{ }^{\circ} \mathrm{C}$, respectively. It should be noted that the Peltier heat 
power $\left(S T_{i} I_{a c}=4.87 \mathrm{~mW}\right.$ for the $50{ }^{\circ} \mathrm{C}$ case $)$ should be far higher than the Joule heat power $\left(I_{a c}{ }^{2}\right.$ $\rho_{T E} L / A=0.095 \mathrm{~mW}$ for the $50^{\circ} \mathrm{C}$ case) to discard possible deviations due to Joule effect.
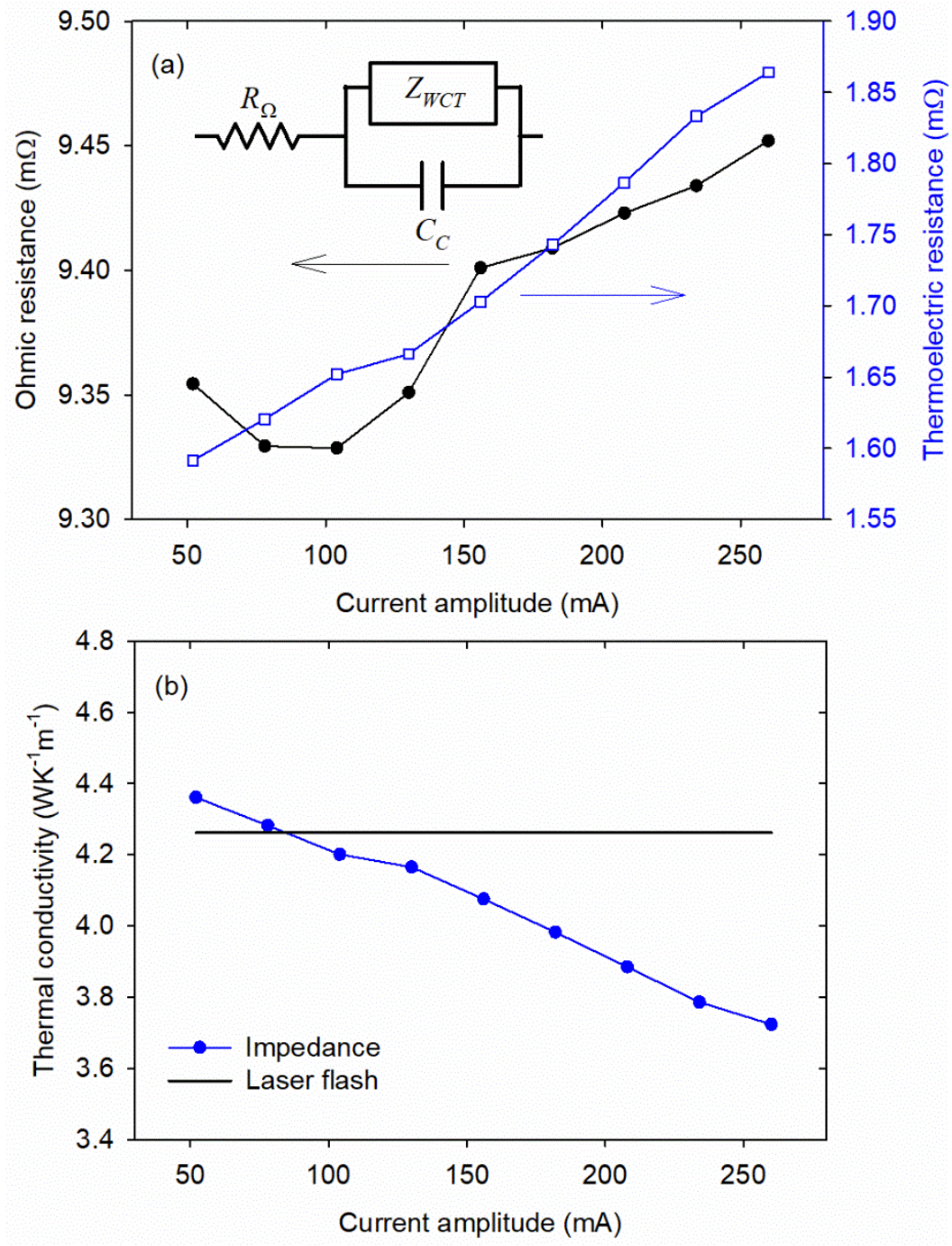

Fig. 3.3. Variation of (a) the ohmic, the thermoelectric resistance, and (b) the extracted thermal conductivity, with different current amplitudes employed in the impedance experiments at $50{ }^{\circ} \mathrm{C}$ of Fig. S3.2. The inset in (a) shows the equivalent circuit used for the fittings.

In Fig. 3.3(a), the values for $R_{\Omega}$ and $R_{T E}$ corresponding to the experiments of Fig. S3.2 are quantified. It can be observed that the ohmic resistance varies randomly at the lower current amplitudes and at values higher than $150 \mathrm{~mA}$, it starts to increase monotonically. This increase could be due to an increase in the electrical resistivity of the TE sample induced by a temperature 
rise due to the heating by Joule effect, which becomes more intense as $I_{a c}$ is increased. On the other hand, it can be observed from Fig. S3.2 that the semicircle in the impedance spectra widens as the current amplitude is increased, which translates into an increase in $R_{T E}$ with $I_{a c}$, as it is shown in Fig. 3.3(a). This increase could be also due to the Joule effect and the increase in the electrical resistivity of the skutterudite material with temperature, but, in addition, an increase in the average Seebeck coefficient, a decrease in the thermal conductivity, and the initial sample temperature, can contribute [see Eq. (3.4)]. Fig. 3.3(b) shows the calculated values of the thermal conductivity from $R_{T E}$ and the Seebeck coefficient values using Eq. (3.7). The latter are provided by measurements from the commercial equipment [see inset of Fig. 3.4(b)]. The thermal conductivity value measured by the laser flash equipment is also shown in Fig. 3.3(b) as reference. It can be observed that good agreement is found for the lower amplitudes $(<110 \mathrm{~mA})$, and higher $I_{a c}$ values lead to significant deviations. The previously optimized value of $104 \mathrm{~mA}$ (corresponding to $1000 \mathrm{~W} / \mathrm{m}^{2}$ Peltier heat power per unit area) lies in the low current amplitude range where the agreement with $\lambda_{T E}$ is good and the $R_{\Omega}$ does not tend to increase, which proves its validity.

We also evaluated the effect of the variation of the current amplitude in the impedance spectra using a $2.08 \mathrm{~mm} \times 2.01 \mathrm{~mm} \times 8.00 \mathrm{~mm} \mathrm{Fe}{ }_{0.95} \mathrm{Co}_{0.05} \mathrm{Si}_{2}$ sample [16], whose electrical resistivity is around 10 times higher than that for the skutterudite material and, moreover, unlike the skutterudite material, it decreases with temperature [see Fig. S3.3(a)]. In this sample, the Joule effect is expected to be more prominent. IS measurements were performed on this material at room temperature and under ambient air conditions (no vacuum) in the $10 \mathrm{mHz}-20 \mathrm{kHz}$ range and employing different current amplitudes. The obtained results can be seen in Fig. S3.3(b). It can be observed that, unlike the case of the skutterudite, a shift of the real part of the impedance signal towards lower values is produced, which is more intense at higher current amplitudes. An explanation of this behavior is again possible by the existence of Joule effect and the connected increase in temperature which decreases the electrical resistivity of this material, and hence its ohmic resistance, which yields a shift of the real impedance towards lower values. On the other hand, a decrease in $R_{T E}$ is observed [see inset of Fig. S3.3(b)], which could be due to the reasons mentioned above but now with a more dominant contribution from the decrease in the electrical 
resistivity with temperature. In this case, it is also important to optimize the current amplitude in order to minimize the observed shifts (Joule effect).

\subsubsection{Characterization by the impedance method}

Using the previously optimized current amplitudes, the skutterudite sample was characterized by IS at different temperatures in the $50-250{ }^{\circ} \mathrm{C}$ range. Five measurement cycles from 50 to 250 ${ }^{\circ} \mathrm{C}$ were measured. Each cycle was initiated with remade contacts. Fig. 3.4(a) shows the impedance spectra obtained for one of these cycles. All the spectra show unnoisy measurements and an excellent fitting (solid lines) to the equivalent circuit of Fig. 3.3(a). Fitting error values $<1 \%$ were obtained for $R_{\Omega}$ and $R_{T E}$ in all cases. It can be observed that even for the spectrum at the lowest temperature, the impedance response is clearly observed. At this temperature, the skutterudite exhibits a lower performance and the equipment is still able to precisely record points which are separated by $\approx 0.1 \mathrm{~m} \Omega$, which demonstrates the capability of this technique to measure materials with modest TE properties.

The TE properties of the skutterudite material were extracted from the average value of the five fitting results of each parameter $\left(R_{\Omega}\right.$ and $\left.R_{T E}\right)$ at each temperature using Eqs. (3.6) to (3.8). Fig. 3.4[(b)-(d)] show the thermal conductivity, electrical resistivity, and the dimensionless figure of merit $z T$ obtained from the IS method, respectively, which are compared with the measurements from commercial equipment. All the properties show a good agreement and reproduce the trends found in the commercial equipment measurements, except the point at the highest temperature $\left(250^{\circ} \mathrm{C}\right)$ from the electrical resistivity. This deviation is attributed to changes experienced by the liquid metal layer employed at the junctions, which tends to solidify at these higher temperatures, and even remains solid when the temperature returns to room values. This introduces a somewhat larger contact resistance which becomes no longer negligible. It should be noted that the very thin $\mathrm{Cu}$ wires which measure the voltage difference are embedded in the junctions (see Fig. 3.2), and hence are in contact with the liquid metal material, which can contribute to the measured resistance if its influence is not kept low. This fact also limits the maximum temperature of the method, since the rest of the elements of the setup can stand for much higher temperature values, so a most suitable solder or liquid metal could increase the capability of the method to measure at higher temperatures. 


\section{Chapter 3}
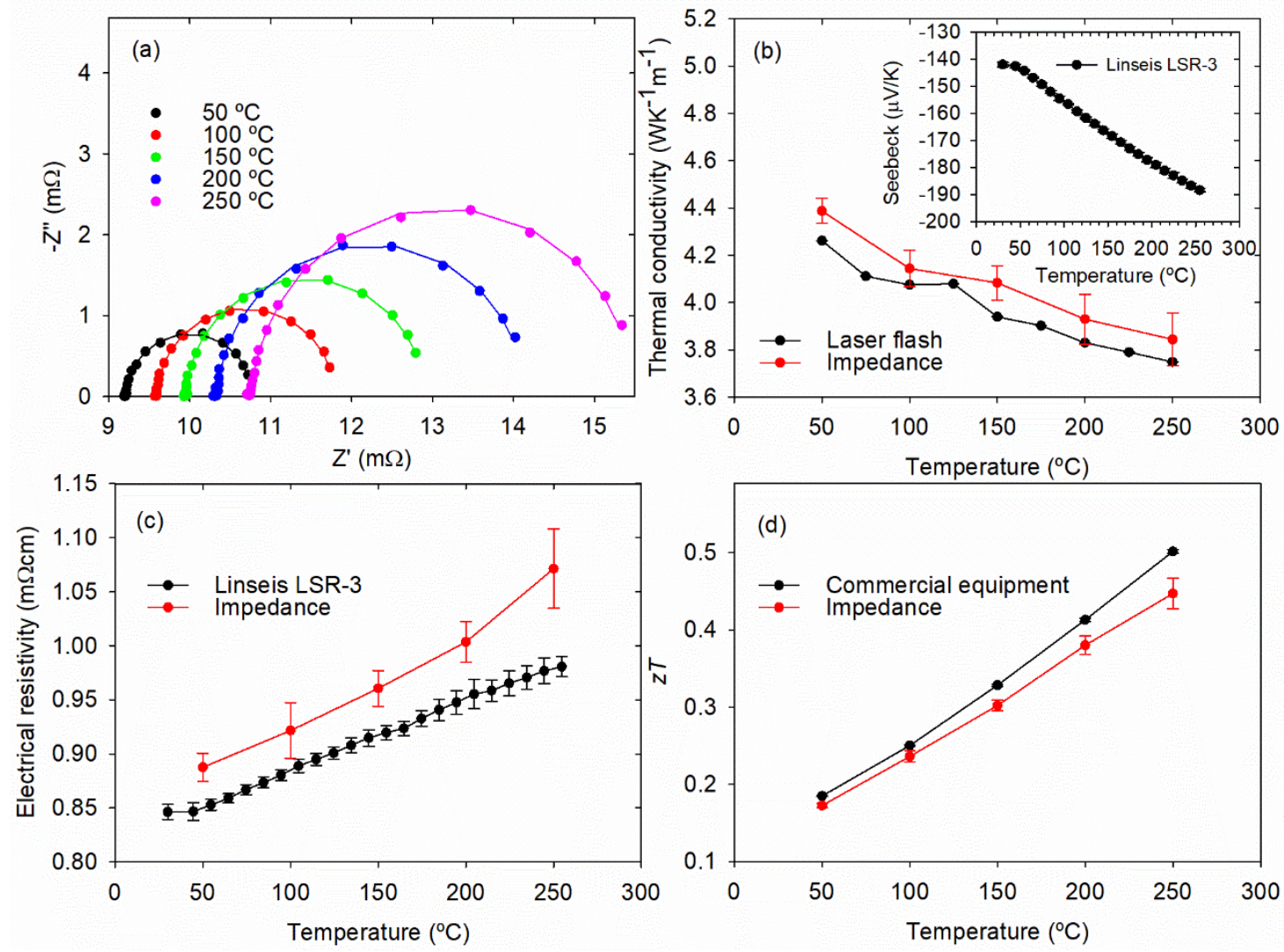

Fig. 3.4. (a) Impedance spectroscopy measurements at different temperatures from one of the five measurement cycles performed. The lines represent the fittings to the experimental values. (b) Thermal conductivity, (c) electrical resistivity and (d) $z T$ values extracted from the impedance method and compared with results from different commercial equipment. The inset in (b) shows the Seebeck coefficient measured by the Linseis LSR-3 equipment, which is required to obtain the thermal conductivity by the impedance method. The error bars account for the total combined random errors, excluding the contribution from the specific heat for the laser flash case.

\subsubsection{Precision and accuracy evaluation}

In order to evaluate the precision and accuracy of the impedance method, random and systematic errors, respectively, were calculated for the thermal conductivity, electrical resistivity and $z T$. The total combined random errors $u_{c}$ of each parameter were calculated using [17]

$$
u_{c}^{2}=\sum_{i=1}^{N}\left(\frac{\partial f_{p}}{\partial x_{i}}\right)^{2} u^{2}\left(x_{i}\right)
$$

with $f_{p}$ being each of the TE properties $\left(\rho_{T E}, \lambda_{T E}\right.$, or $\left.z T\right)$, and $x_{i}$ being each of the parameters required for the determination of the corresponding TE property with its associated error $u$. The 
random errors for the thermal conductivity were calculated taking into account (i) the standard deviation from three measurements performed using the commercial equipment for the Seebeck coefficient, (ii) the uncertainty of the thermocouple $\left[u\left(T_{i}\right)=1^{\circ} \mathrm{C}\right]$, (iii) the uncertainty in the length of the sample which was measured using a caliber $[u(L)=0.005 \mathrm{~mm}]$, (iv) the uncertainty in the area of the sample, and (v) the standard deviation of the five measurements at each temperature to obtain the average value of $R_{T E}$. The contribution from the fitting errors in $R_{T E}$ (which were $<1 \%$ ) was neglected since it was negligible in comparison with the standard deviation. From Table S3.1, which shows all these contributions, it can be observed that the contributions from the Seebeck coefficient and $R_{T E}$ are the most significant, being the rest negligible.

The random errors for the electrical resistivity were calculated taking into account (i) the uncertainty in the length of the sample, (ii) the uncertainty in the area, and (iii) the standard deviation from the five measurements at each temperature to obtain the average $R_{\Omega}$. It should be noticed that the latter contribution is the most significant, as shown in Table S3.1. As occurred for $R_{T E}$, the contribution of the fitting errors $(<1 \%)$ for $R_{\Omega}$ was neglected. Finally, the random errors for $z T$ were calculated from the contributions of the standard deviations of both $R_{\Omega}$ and $R_{T E}$. In this case, both show similar contributions (see Table S3.1). The error bars shown in Fig. 3.4 correspond to the calculated combined random errors. In the case of results from the Seebeck coefficient and the electrical resistivity using commercial equipment, the random errors were obtained from the standard deviations of three consecutive measurements performed at each temperature. The combined random error from the thermal conductivity determined by the laser flash method was calculated using Eq. (3.9) taking into account the standard deviation obtained from three consecutive measurements of the thermal diffusivity and the density. The contribution from the specific heat was not available. Systematic errors $u_{s}$ were calculated for the TE properties considering as true values the results obtained from the commercial equipment. It should be noted that a more rigorous calculation should be performed using standard reference materials (SRM), however, there are no SRM available which could provide simultaneously the three properties measured in this study [3].

Table 3.1 shows the average values of each TE property with their associated random, systematic and total errors $u_{T}$, the latter obtained as $u_{T}=\left(u_{c}^{2}+u_{s}^{2}\right)^{0.5}$. Random errors $<3 \%$ are obtained for $\lambda_{T E}$ and $\rho_{T E}$, except at $250{ }^{\circ} \mathrm{C}$ for the latter, which are somewhat higher due to the 
reasons previously mentioned. These low values of the random errors demonstrate the excellent precision of the method. For the case of $z T$, the precision is also excellent, with random errors $\approx 3 \%$, except for the case at $250{ }^{\circ} \mathrm{C}$ due to the higher error in $\rho_{T E}$ at this temperature.

Table 3.1. Average values with their associated random, systematic and total errors of the thermoelectric properties of a skutterudite sample obtained by the impedance spectroscopy method.

\begin{tabular}{cccccc}
\hline & $\begin{array}{c}\text { Temperature } \\
\left({ }^{\circ} \mathbf{C}\right)\end{array}$ & Mean value & $\begin{array}{c}\text { Systematic } \\
\text { error }(\%)\end{array}$ & $\begin{array}{c}\text { Random } \\
\text { error }(\%)\end{array}$ & $\begin{array}{c}\text { Total error } \\
(\boldsymbol{\%})\end{array}$ \\
\hline \multirow{3}{*}{$\begin{array}{c}\text { Thermal } \\
\text { conductivity } \\
\left(\lambda_{T E}\right)\end{array}$} & 50 & $4.39 \mathrm{WK}^{-1} \mathrm{~m}^{-1}$ & 2.95 & 1.21 & 3.19 \\
& 100 & $4.14 \mathrm{WK}^{-1} \mathrm{~m}^{-1}$ & 1.69 & 1.88 & 2.53 \\
& 150 & $4.08 \mathrm{WK}^{-1} \mathrm{~m}^{-1}$ & 3.64 & 1.76 & 4.04 \\
& 200 & $3.93 \mathrm{WK}^{-1} \mathrm{~m}^{-1}$ & 2.60 & 2.69 & 3.74 \\
& 250 & $3.84 \mathrm{WK}^{-1} \mathrm{~m}^{-1}$ & 2.54 & 2.90 & 3.85 \\
\hline $\begin{array}{c}\text { Electrical } \\
\text { resistivity }\end{array}$ & 50 & $0.89 \mathrm{~m} \Omega \mathrm{cm}$ & 4.08 & 1.45 & 4.33 \\
$(\boldsymbol{\rho}$ TE $)$ & 100 & $0.92 \mathrm{~m} \Omega \mathrm{cm}$ & 4.20 & 2.78 & 5.04 \\
& 150 & $0.96 \mathrm{~m} \Omega \mathrm{cm}$ & 4.87 & 1.72 & 5.17 \\
& 200 & $1.00 \mathrm{~m} \Omega \mathrm{cm}$ & 5.90 & 1.90 & 6.20 \\
& 250 & $1.07 \mathrm{~m} \Omega \mathrm{cm}$ & 9.41 & 3.43 & 10.02 \\
\hline $\begin{array}{c}\text { Figure of } \\
\text { merit }(z \boldsymbol{T})\end{array}$ & 150 & 0.173 & 6.67 & 1.59 & 6.86 \\
& 100 & 0.236 & 5.62 & 3.22 & 6.48 \\
& 200 & 0.302 & 7.99 & 2.30 & 8.32 \\
\hline
\end{tabular}

The systematic errors (below $250{ }^{\circ} \mathrm{C}$ ) are $<4 \%$, between $4 \%$ and $6 \%$, and $<8 \%$, for $\lambda_{T E}, \rho_{T E}$, and $z T$, respectively (see Table 3.1), which demonstrates a good agreement with the characterization performed by commercial equipment. The total errors, found from the contribution of the random and systematic errors, are (excluding the case at $250{ }^{\circ} \mathrm{C}$ ) $\approx 4 \%$, between $4.3 \%$ and $6.2 \%$, and $\left\langle 9 \%\right.$, for $\lambda_{T E}, \rho_{T E}$ and $z T$, respectively (see Table 3.1 ), which proves the suitability to accurately characterize bulk TE materials by the impedance method. Especially, the capability to determine the thermal conductivity with excellent precision and accuracy is remarkable, since it represents an appropriate alternative to the laser flash method, which typically exhibits higher errors and requires the measurement of the density and the specific heat, which are not needed to obtain the $z T$. It should be noted that, as we mentioned above, the error bars from the laser flash results in Fig. 3.4(b) do not include the error contribution from the specific 
heat, which is usually between $\pm 4 \%$ but can show occasionally much higher variations as shown in a previously conducted round robin campaign [18].

Finally, it is important to recall that low errors are only obtained when the sample and the contacts are properly cut and cleaned, and the liquid metal covers the whole surface. To minimize the electrical and thermal contacts is key to reach a nice impedance response.

\subsection{Conclusions}

The possibility to determine the electrical resistivity, thermal conductivity (if the Seebeck coefficient is known), and the dimensionless figure of merit $z T$ of a bulk TE material by IS has been demonstrated for a low-performance TE material up to $250{ }^{\circ} \mathrm{C}$. A new setup was developed to measure TE materials in a 4-probe mode with the possibility of varying the ambient temperature. A skutterudite material, which shows low performance at room temperature, was characterized by the impedance method. A clear impedance signal and suitable characterization were obtained even at the lowest temperature, which demonstrates the capability of the method to test low- $z T$ materials. All the TE properties of the skutterudite sample were determined by fittings performed to the experimental impedance spectra employing a suitable equivalent circuit. It was found to be important to optimize the ac current amplitude to employ in the impedance experiments, since significant variations in the impedance spectra can occur, probably due to the Joule effect and/or the dependence of the thermoelectric properties with temperature. Random errors were calculated by performing five measurements at each temperature with remade contacts, showing an excellent precision of the method (random errors $<4.5 \%$ for all properties). Systematic errors were also determined by comparison with measurements of the sample using commercial equipment, resulting in values $<4 \%$, between $4 \%$ and $6 \%$, and $<8 \%$, for $\lambda_{T E}, \rho_{T E}$ and $z T$, respectively, which proves the good accuracy of the method. It is especially remarkable the excellent results found for the characterization of the thermal conductivity, which establishes the impedance method as an alternative approach to the laser flash method, which typically exhibits higher errors and requires additional measurements (density and specific heat), which are not needed to obtain the $z T$ and which are not necessary in the impedance approach. 


\section{Acknowledgements}

B.B.P. and J.G.C. acknowledge financial support from the Spanish Agencia Estatal de Investigación under the Ramón y Cajal program (RYC-2013-13970), from the Universitat Jaume I under the Project No. UJI-A2016-08, and the technical support of Raquel Oliver Valls and José Ortega Herreros. A.V.P. and J.P.G. wish to thank the UK Engineering and Physical Sciences Research Council (EP/K019767/1) for financial support.

\section{References}

[1] J.R. Sootsman, D.Y. Chung, M.G. Kanatzidis, New and Old Concepts in Thermoelectric Materials, Angew. Chemie Int. Ed. 48 (2009) 8616-8639. https://doi.org/10.1002/anie.200900598.

[2] W.J. Parker, R.J. Jenkins, C.P. Butler, G.L. Abbott, Flash method of determining thermal diffusivity, heat capacity, and thermal conductivity, J. Appl. Phys. 32 (1961) 1679-1684. https://doi.org/10.1063/1.1728417.

[3] K.A. Borup, J. de Boor, H. Wang, F. Drymiotis, F. Gascoin, X. Shi, L. Chen, M.I. Fedorov, E. Müller, B.B. Iversen, G.J. Snyder, Measuring thermoelectric transport properties of materials, 8 (2015) 423-435. https://doi.org/10.1039/C4EE01320D.

[4] A.D. Downey, T.P. Hogan, B. Cook, Characterization of thermoelectric elements and devices by impedance spectroscopy, Rev. Sci. Instrum. 78 (2007) 093904. https://doi.org/10.1063/1.2775432.

[5] A. De Marchi, V. Giaretto, An accurate new method to measure the dimensionless figure of merit of thermoelectric devices based on the complex impedance porcupine diagram, Rev. Sci. Instrum. 82 (2011). https://doi.org/10.1063/1.3656074.

[6] J. García-cañadas, G. Min, Impedance spectroscopy models for the complete characterization of thermoelectric materials, J. Appl. Phys. 116 (2014). https://doi.org/10.1063/1.4901213.

[7] C.Y. Yoo, Y. Kim, J. Hwang, H. Yoon, B.J. Cho, G. Min, S.H. Park, Impedance spectroscopy for assessment of thermoelectric module properties under a practical 
Chapter 3

$\begin{array}{lllll}\text { operating } & \text { temperature, } & \text { Energy. } & 152 & \text { (2018) }\end{array}$ https://doi.org/10.1016/j.energy.2017.12.014.

[8] Y. Apertet, H. Ouerdane, Small-signal model for frequency analysis of thermoelectric systems, Energy Convers. Manag. $149 \quad$ (2017) 564-569. https://doi.org/10.1016/J.ENCONMAN.2017.07.061.

[9] X. Yuan, H. Wang, J. Colinsun, J. Zhang, J. Colin Sun, J. Zhang, AC impedance technique in PEM fuel cell diagnosis-A review, Int. J. Hydrogen Energy. 32 (2007) 4365-4380. https://doi.org/10.1016/J.IJHYDENE.2007.05.036.

[10] R. Kötz, M. Hahn, R. Gallay, Temperature behavior and impedance fundamentals of $\begin{array}{llcccc}\text { supercapacitors, } & \text { J. } & \text { Power } & \text { Sources. } & 154 & \text { (2006) 550-555. }\end{array}$ https://doi.org/10.1016/J.JPOWSOUR.2005.10.048.

[11] S.A. Grammatikos, R.J. Ball, M. Evernden, R.G. Jones, Impedance spectroscopy as a tool for moisture uptake monitoring in construction composites during service, Compos. Part A Appl. Sci. Manuf. $105 \quad$ (2018) 108-117. https://www.sciencedirect.com/science/article/pii/S1359835X17304050?via\%3Dihub (accessed October 29, 2018).

[12] G.W. Walter, A review of impedance plot methods used for corrosion performance analysis of painted metals, Corros. Sci. 26 (1986) 681-703. https://doi.org/10.1016/0010938X(86)90033-8.

[13] F. Fabregat-Santiago, G. Garcia-Belmonte, I. Mora-Seró, J. Bisquert, Characterization of nanostructured hybrid and organic solar cells by impedance spectroscopy, Phys. Chem. Chem. Phys. 13 (2011). https://doi.org/10.1039/c0cp02249g.

[14] J. García-cañadas, G. Min, Thermoelectric Material Devices, R. Soc. Chem. Cambridge. (2016).

[15] J. García-Cañadas, G. Min, Thermal dynamics of thermoelectric phenomena from frequency resolved methods, AIP Adv. 6 (2016) 035008. https://doi.org/10.1063/1.4943958.

[16] P. Ziolkowski, C. Stiewe, J. de Boor, I. Druschke, K. Zabrocki, F. Edler, S. Haupt, J. 
König, E. Mueller, Iron Disilicide as High-Temperature Reference Material for Traceable Measurements of Seebeck Coefficient Between 300 K and 800 K, J. Electron. Mater. 46 (2017) 51-63. https://doi.org/10.1007/s11664-016-4850-5.

[17] Evaluation of measurement data-Guide to the expression of uncertainty in measurement, (1995). https://www.bipm.org/en/publications/guides/gum.html (accessed October 23, 2018).

[18] H. Wang, W.D. Porter, H. Böttner, J. König, L. Chen, S. Bai, T.M. Tritt, A. Mayolet, J. Senawiratne, C. Smith, F. Harris, P. Gilbert, J. Sharp, J. Lo, H. Kleinke, L. Kiss, Transport properties of bulk thermoelectrics: An international round-robin study, part II: Thermal diffusivity, specific heat, and thermal conductivity, J. Electron. Mater. 42 (2013) 10731084. https://doi.org/10.1007/s11664-013-2516-0. 


\section{Supplementary information}

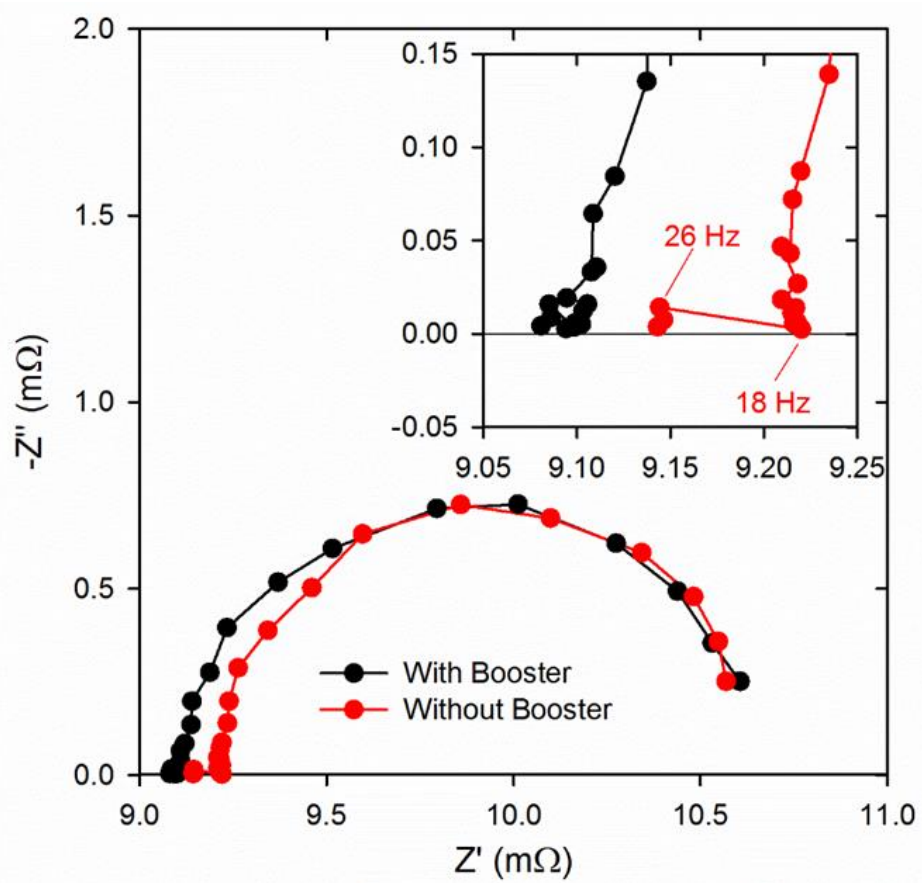

Fig. S3.1. Impedance spectra performed in vacuum at room temperature at $120 \mathrm{~mA}$ current amplitude with Booster (black) and without Booster (red). The inset shows the high frequency magnification, where the jump in the real part of the impedance is more clearly observed when the Booster is not used. A new equipment will be launched soon by Metrohm Autolab which could solve this issue. 
Chapter 3
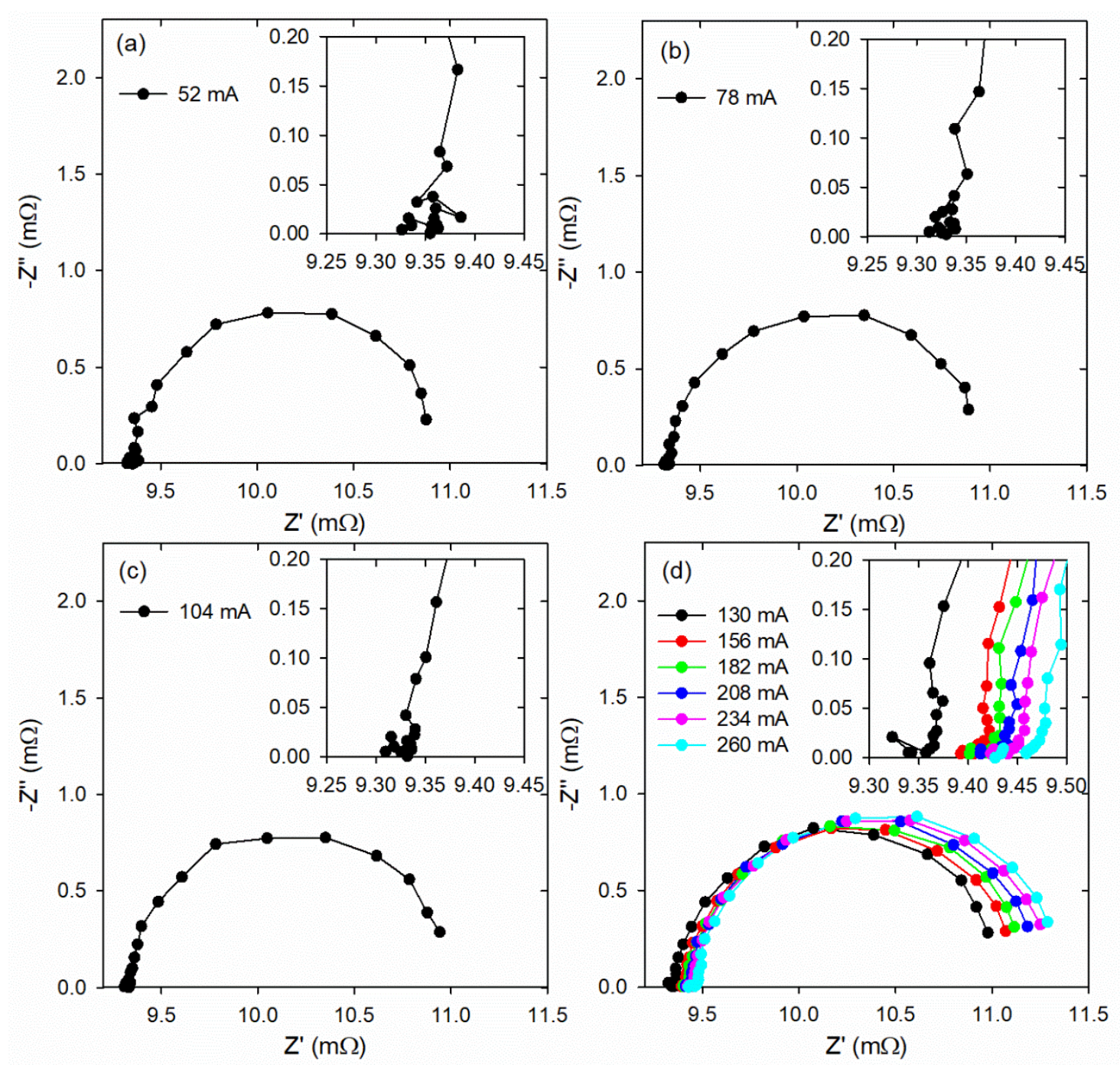

Fig. S3.2. Impedance spectra at (a) $52 \mathrm{~mA}$, (b) $78 \mathrm{~mA}$, (c) $104 \mathrm{~mA}$, and (d) 130 to $26 \mathrm{~mA}$ current amplitudes $I_{a c}$ at $50{ }^{\circ} \mathrm{C}$ ambient temperature under vacuum. The current amplitudes from 52 to $260 \mathrm{~mA}$ match with Peltier heat power per unit area $\left(S T_{i} I_{a c} / \mathrm{A}\right)$ generated at the junctions of $500,750,1000,1250,1500,1750$, 2000,2250 , and $2500 \mathrm{~W} / \mathrm{m}^{2}$. The inset of each figure shows a magnification of the high frequency part. 
Chapter 3
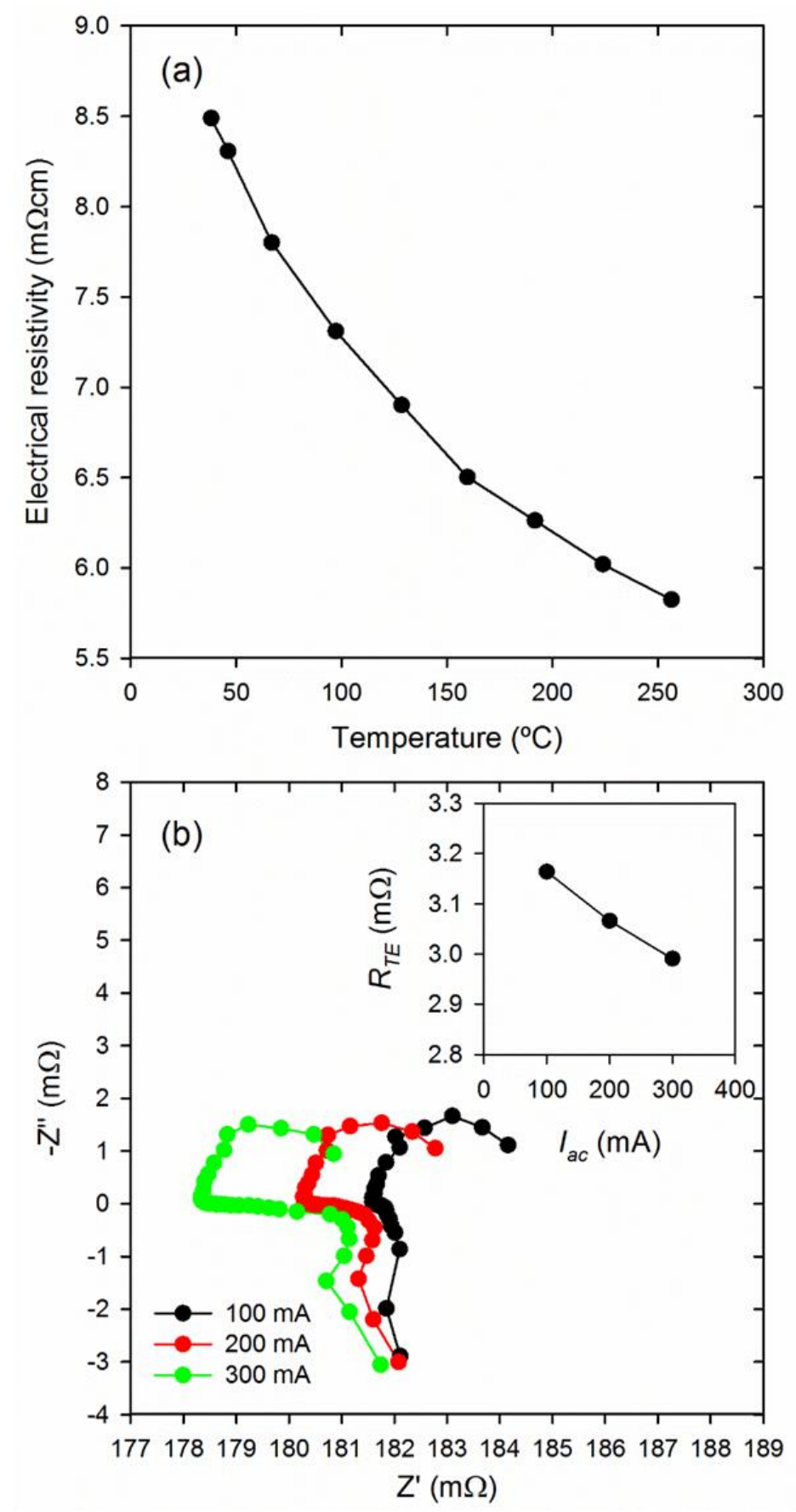

Fig. S3.3. (a) Electrical resistivity of the $\mathrm{Fe}_{0.95} \mathrm{Co}_{0.05} \mathrm{Si}_{2}$ sample as a function of temperature. (b) Impedance spectra at room conditions of the $\mathrm{Fe}_{0.95} \mathrm{Co}_{0.05} \mathrm{Si}_{2}$ sample at different current amplitudes. The inset shows the variation of the thermoelectric resistance $R_{T E}$ with the applied current amplitudes $I_{a c}$. 
Chapter 3

Table S3.1. Contributions to the total combined random error of each of the thermoelectric properties determined by the impedance method.

\begin{tabular}{|c|c|c|c|c|c|c|}
\hline & & $50^{\circ} \mathrm{C}$ & $100^{\circ} \mathrm{C}$ & $150^{\circ} \mathrm{C}$ & $200^{\circ} \mathrm{C}$ & $250^{\circ} \mathrm{C}$ \\
\hline \multirow{5}{*}{$\begin{array}{c}\text { Contributions } \\
\text { to random } \\
\text { error of } \lambda_{T E} \\
\left(\mathbf{W}^{2} \mathbf{K}^{-2} \mathbf{m}^{-2}\right)\end{array}$} & $\left(\frac{\partial \lambda_{T E}}{\partial S}\right)^{2} u^{2}(S)$ & $1.60 \times 10^{-3}$ & $1.23 \times 10^{-3}$ & $1.03 \times 10^{-3}$ & $8.45 \times 10^{-4}$ & $7.28 \times 10^{-4}$ \\
\hline & $\left(\frac{\partial \lambda_{T E}}{\partial T_{i}}\right)^{2} u^{2}\left(T_{i}\right)$ & $1.85 \times 10^{-4}$ & $1.23 \times 10^{-4}$ & $9.32 \times 10^{-5}$ & $6.90 \times 10^{-5}$ & $5.40 \times 10^{-5}$ \\
\hline & $\left(\frac{\partial \lambda_{T E}}{\partial L}\right)^{2} u^{2}(L)$ & $1.92 \times 10^{-5}$ & $1.71 \times 10^{-5}$ & $1.66 \times 10^{-5}$ & $1.54 \times 10^{-5}$ & $1.47 \times 10^{-5}$ \\
\hline & $\left(\frac{\partial \lambda_{T E}}{\partial A}\right)^{2} u^{2}(A)$ & $9.88 \times 10^{-5}$ & $8.82 \times 10^{-5}$ & $8.56 \times 10^{-5}$ & $7.93 \times 10^{-5}$ & $7.59 \times 10^{-5}$ \\
\hline & $\left(\frac{\partial \lambda_{T E}}{\partial R_{T E}}\right)^{2} u^{2}\left(R_{T E}\right)$ & $9.07 \times 10^{-4}$ & $4.58 \times 10^{-3}$ & $3.94 \times 10^{-3}$ & $1.02 \times 10^{-2}$ & $1.15 \times 10^{-2}$ \\
\hline \multirow{3}{*}{$\begin{array}{l}\text { Contributions } \\
\text { to random } \\
\text { error of } \rho_{T E} \\
\left(\mathbf{m} \Omega^{2} \mathbf{c m}^{2}\right)\end{array}$} & $\left(\frac{\partial \rho_{T E}}{\partial L}\right)^{2} u^{2}(L)$ & $7.85 \times 10^{-17}$ & $8.46 \times 10^{-17}$ & $9.19 \times 10^{-17}$ & $1.00 \times 10^{-16}$ & $1.14 \times 10^{-16}$ \\
\hline & $\left(\frac{\partial \rho_{T E}}{\partial A}\right)^{2} u^{2}(A)$ & $4.04 \times 10^{-16}$ & $4.36 \times 10^{-16}$ & $4.74 \times 10^{-16}$ & $5.17 \times 10^{-16}$ & $5.89 \times 10^{-16}$ \\
\hline & $\left(\frac{\partial \rho_{T E}}{\partial R_{\Omega}}\right)^{2} u^{2}\left(R_{\Omega}\right)$ & $1.61 \times 10^{-14}$ & $6.53 \times 10^{-14}$ & $2.68 \times 10^{-14}$ & $3.57 \times 10^{-14}$ & $1.34 \times 10^{-13}$ \\
\hline \multirow{2}{*}{$\begin{array}{c}\text { Contributions } \\
\text { to random } \\
\text { error of } z T\end{array}$} & $\left(\frac{\partial z T}{\partial R_{\Omega}}\right)^{2} u^{2}\left(R_{\Omega}\right)$ & $6.10 \times 10^{-6}$ & $4.29 \times 10^{-5}$ & $2.65 \times 10^{-5}$ & $5.12 \times 10^{-5}$ & $2.34 \times 10^{-4}$ \\
\hline & $\left(\frac{\partial z T}{\partial R_{T E}}\right)^{2} u^{2}\left(R_{T E}\right)$ & $1.40 \times 10^{-6}$ & $1.49 \times 10^{-5}$ & $2.16 \times 10^{-5}$ & $9.51 \times 10^{-5}$ & $1.56 \times 10^{-4}$ \\
\hline
\end{tabular}


4. Complete characterization of thermoelectric materials by impedance spectroscopy 


\title{
Complete characterization of thermoelectric materials by impedance spectroscopy
}

\author{
Braulio Beltrán-Pitarch ${ }^{1}$, Jesús Prado-Gonjal ${ }^{2}$, Anthony V. Powell ${ }^{2}$, Jorge García- \\ Cañadas ${ }^{1, *}$
}

\footnotetext{
${ }^{1}$ Department of Industrial Systems Engineering and Design, Universitat Jaume I, Campus del Riu Sec, 12071 Castellón, Spain

${ }^{2}$ Department of Chemistry, University of Reading, RG6 6AD, Reading, UK

*e-mail: garciaj@uji.es
} 


\begin{abstract}
Thermoelectric materials can directly convert waste heat into electricity. Due to the vast amount of energy available as waste heat in our society, these materials could contribute to reduce our dependence on fossil fuels and their associated environmental problems. However, the heat to electricity conversion efficiency of thermoelectric materials is still a limiting factor, and extensive efforts are being undertaken to improve their performance. The search for more efficient materials is focused on the optimization of three properties (Seebeck coefficient, electrical resistivity, and thermal conductivity). Typically, these are determined as function of temperature through independent measurements on two or more instruments, making thermoelectric characterization tedious and time consuming, which complicates the attainment of a more efficient heat to electricity energy conversion. Here, it is demonstrated for the first time that a complete thermoelectric characterization of a material may be achieved from a single electrical measurement performed on one instrument only, by employing the impedance spectroscopy (IS) method. A skutterudite sample is used for the demonstration, which is sandwiched between two stainless steel contacts in a four-probe arrangement and their properties are determined from 50 to $250^{\circ} \mathrm{C}$. This new approach shows good precision and agrees with characterization of the same sample performed with commercial equipment, illustrating the power of the technique to facilitate the rapid and efficient evaluation of thermoelectric materials.
\end{abstract}




\section{Graphical abstract}
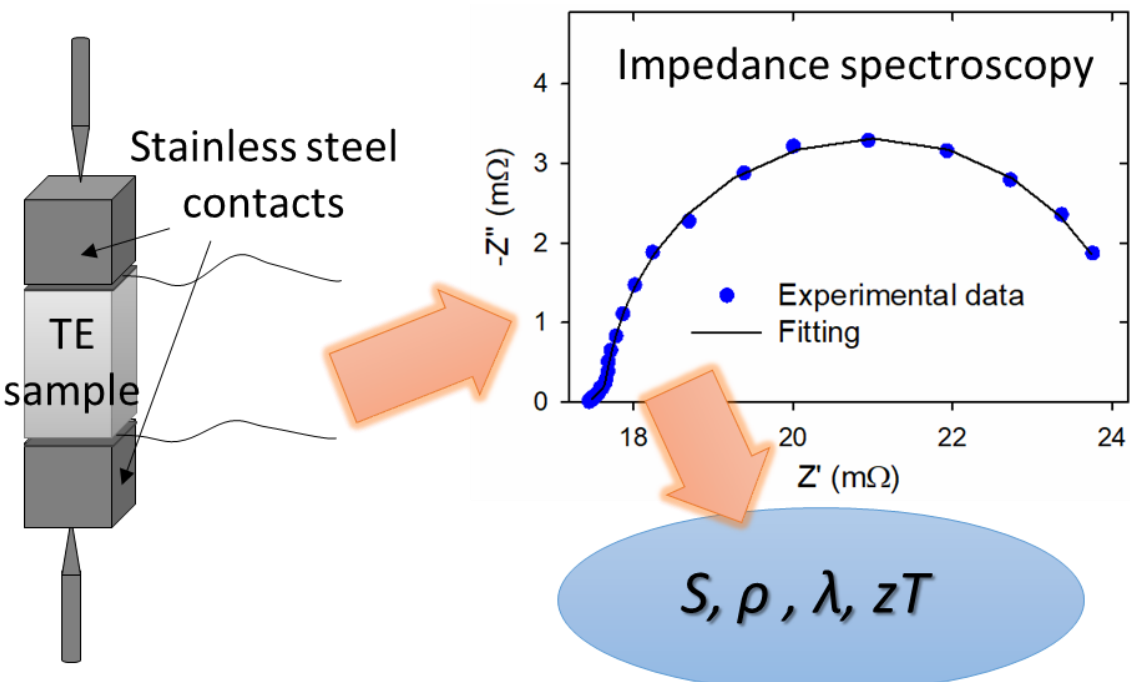

Fig. 4.1. Graphical abstract of the work: Complete Characterization of Thermoelectric Materials by Impedance Spectroscopy. 


\subsection{Introduction}

Nowadays, more than $60 \%$ of the global power is lost as waste heat, which represents $\approx 15$ TW. A $10 \%$ recovery of this energy will exceed the summation of most current renewable energy sources (solar, wind, geothermal, and hydro energy) [1]. Thermoelectric (TE) materials can directly convert waste heat into electricity. Due to this, they have interest in applications such as automobiles and industries, where they can generate energy from the waste heat released by exhaust gases and reduce $\mathrm{CO}_{2}$ emissions [2]. They can also convert solar warmth into electricity when integrated in solar thermoelectric generators [3]. In addition, they are also potentially able to power wearable electronics and sensors using environmental heat or from human bodies, being a top candidate for self-powering sensors from the internet of things, empowering the elimination of batteries, which are toxic and subjected to frequent recharging and replacement [4]. An efficient heat to electricity energy conversion from these applications would help to reduce our dependence on fossil fuels and their associated environmental problems.

However, the efficiency of current TE materials is still limited. The search for more efficient materials is guided by the optimization of three properties, the electrical conductivity $\sigma$, the Seebeck coefficient $S$, and the thermal conductivity $\lambda$ (which is the addition of the lattice thermal conductivity and the electronic thermal conductivity). These define a dimensionless figure of merit $z T=\sigma S^{2} T / \lambda, T$ is the absolute temperature, which is related to the materials efficiency [5]. $z T$ is typically obtained by the independent measurement as a function of temperature of $\sigma, S$, and $\lambda$. This usually requires at least two different instruments. $S$ and $\sigma$ can be measured using a single apparatus, while the most frequently used method to determine $\lambda$ is the laser flash technique, which provides the thermal diffusivity $\alpha$. Thus, knowledge of the specific heat $C_{p}$ and the mass density $d$ is required, since $\lambda=\alpha d C p$ [6]. The measurement of the specific heat requires an additional measurement, performed either by using the same laser flash equipment or another instrument. For the mass density, an Archimedes balance is frequently employed. The significant number of instruments required, each with their own sources of error, and the large number of measurements to be performed, makes the task of completely characterization of TE materials quite tedious and time consuming. In addition, much of the required equipment is quite expensive, and hence not always readily accessible to all researchers. All these disadvantages entail 
significant obstacles in the search for better TE materials, eventually affecting the attainment of a more efficient heat to electricity energy conversion.

Several techniques have been developed which allow the complete characterization of TE materials [7-11]. Here a new method is proposed, which unlike the previously reported techniques does not require two identical samples nor the creation of a temperature gradient across the sample, the measurement of which can introduce significant uncertainty [12]. In addition, the determination of the TE properties does not require a series of measurements, as all properties can be extracted from a single measurement. To our knowledge, this is the first time that all these advantages are offered by a measurement technique. The method is based on the measurement of the impedance signal of a TE sample that is sandwiched by a material of known thermal conductivity. Although the application of impedance spectroscopy (IS) to thermoelectricity dates back from the 2000s [13,14], this approach was proposed by us in 2014 [15], and it has only been demonstrated to date in TE modules [16-18]. Here the approach is applied to a skutterudite material, for which complete TE characterization is achieved up to $250{ }^{\circ} \mathrm{C}$ (although with some deviations in $\sigma$ at the higher temperatures). The results are compared with the values of the TE properties determined using commercially available equipment, and the random and systematic errors are calculated. The fact that the new method is based on IS introduces additional advantages, since it is a widely used technique in many fields of research (solar cells $[19,20]$, fuel cells [21], supercapacitors [22], corrosion [23], electroceramics [24], etc.). For this reason, highly reliable impedance equipment exists in the market and can be found in many research institutions, which makes the method more accessible.

\subsection{Experimental setup}

The setup employed for the complete characterization of the skutterudite sample is shown in Fig. 4.2. It is similar to the setup employed in our previous work to characterize TE materials of known Seebeck coefficient [25]. Unlike the previously reported setup, the TE sample $\left(\mathrm{CoSb}_{2.75} \mathrm{Sn}_{0.05} \mathrm{Te}_{0.20}\right.$ skutterudite [26] of $\left.1.85 \mathrm{~mm} \times 2.13 \mathrm{~mm} \times 6.95 \mathrm{~mm}\right)$ is here sandwiched by two stainless steel (AISI 304) contacts of the same cross-sectional area and $2 \mathrm{~mm}$ thickness. A four-probe arrangement is employed (see inset of Fig. 4.2), where the current is injected and extracted by two sharpened stainless steel screws and the voltage is measured across the sample 
by inserting very thin (15 $\mu \mathrm{m}$ diameter, Alfa Aesar) tungsten wires at the junctions. These wires are used instead of the $\mathrm{Cu}$ wires employed in our previous study since reactions with the stainless steel were observed for copper at higher temperatures. In order to minimize the electrical and thermal contact resistances, a layer of a liquid metal $\left(\mathrm{Ga}_{62} \mathrm{In}_{22} \mathrm{Sn}_{16}\right.$, Alfa Aesar) is homogeneously spread at the junctions (see inset of Fig. 4.2).

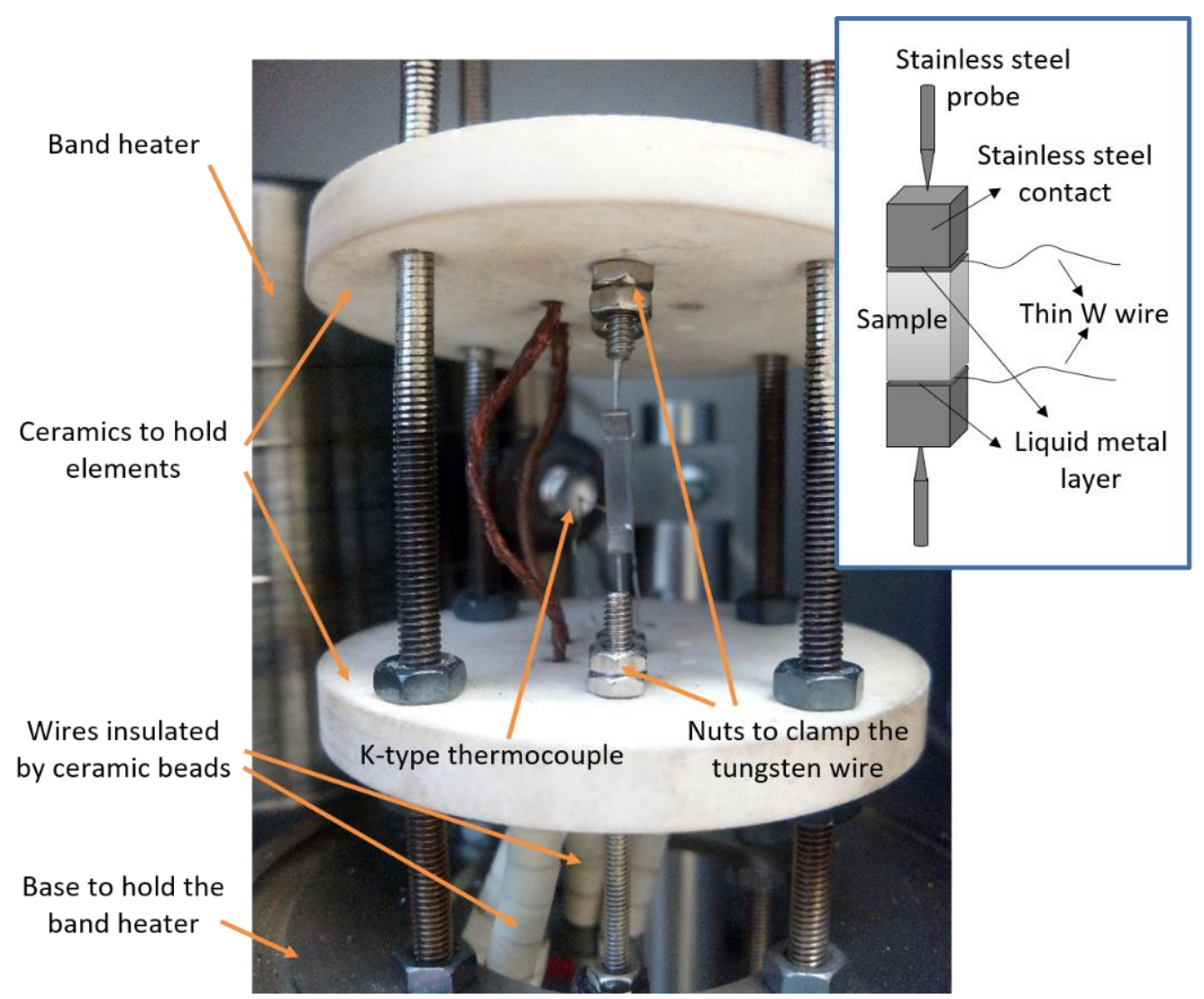

Fig. 4.2. Photograph of the sample holder employed. A schematic description of how the sample is contacted is provided in the inset.

The two stainless-steel screws which drive the current are held by nuts at holed ceramics (Macor, Corning) which provide electrical insulation, as shown in Fig. 4.2. These screws are connected to thick copper wires insulated by ceramic beads. Stainless steel screws were chosen due to their low thermal conductivity $\left(\approx 14 \mathrm{~W} / \mathrm{K}^{-1} \mathrm{~m}^{-1}\right)$, which reduces heat losses by conduction. They were also sharpened for the same purpose. The very thin tungsten wires that measure the potential difference are clamped at the sample holder by two nuts screwed with stainless steel screws, which are held by the ceramic plates (see Fig. 4.2). These screws are also connected to 
thick copper wires insulated by ceramic beads. The bottom holed ceramic disc is fixed at four threaded studs by nuts, while the top ceramic is free to move to be able to allocate samples of different lengths, and additionally provide pressure to the contacts. A stainless-steel base is also held by nuts at the studs. This base is used to hold a band heater (Ref. MB2E2JN1-B12, Watlow) which surrounds the sample holder and is used to provide different ambient temperatures. The ambient temperature is measured by a K-type thermocouple (RS) placed close to the TE sample (see Fig. 4.2), whose temperature is controlled by a temperature controller (Watlow EZ Zone PM) which powers the heater.

All the impedance measurements were performed inside a stainless-steel vacuum chamber at pressure values $<10^{-4} \mathrm{mbar}$ in order to eliminate convection heat losses. In addition, the metallic vacuum chamber also serves as a Faraday cage, which reduces electromagnetic noise during the measurements. The TE sample used in this study was an isotropic n-type skutterudite $\left(\mathrm{CoSb}_{2.75} \mathrm{Sn}_{0.05} \mathrm{Te}_{0.20}\right)$, which was cut with a diamond saw of $0.3 \mathrm{~mm}$ diameter from an original disc shape. A careful and suitable cutting is important to obtain a crack free sample of highly uniform cross-sectional area. The skutterudite sample was characterized using commercial equipment in its disc shape before performing the impedance measurements. A Linseis LSR-3 equipment was used to determine the electrical resistivity and the Seebeck coefficient. For the thermal conductivity a Netzsch LFA 447 laser flash apparatus was employed. The specific heat of the sample was determined using the same equipment via a comparative method using a Pyroceram reference sample. The density of the sample, which is also required for the determination of the thermal conductivity by the laser flash method, was measured using an Archimedes balance.

A PGSTAT30 potentiostat (Metrohm Autolab B.V.) equipped with a FRA2 impedance module and a BOOSTER10A, was used to perform the IS measurements. The potentiostat was controlled by the Nova 1.11 software. At each temperature the impedance measurement was conducted in 40 logarithmically distributed frequency steps between $5 \mathrm{mHz}$ and $500 \mathrm{~Hz}$. The measurements were performed using a maximum integration time of $10 \mathrm{~s}$ and 2 minimum integration cycles. The fitting to the impedance spectra were performed using Zview software. In our previous paper it was discussed the use of the current booster to reduce a systematic jump in the real impedance produced due to a change in the gain of the equipment, which occurs at 
frequencies around $25 \mathrm{~Hz}$. Although this jump can be significantly reduced if measurements are performed in the largest possible current range, it distorts the spectra and due to this the fittings are performed discarding the points of frequencies higher than that of the discontinuity.

\subsection{Results and discussion}

\subsubsection{The equivalent circuit}

To obtain all the TE properties from the impedance data obtained in this work, the experimental spectra were fitted using the equivalent circuit corresponding to a TE material sandwiched between two metallic contacts [15]. This equivalent circuit consists of an ohmic resistance $R_{\Omega}$ connected in series with the parallel combination of a constant temperature Warburg $Z_{W C T}$ and an adiabatic Warburg $Z_{W a}$. Each of these elements are given by,

$$
\begin{aligned}
& R_{\Omega}=\frac{\rho_{T E} L}{A} \\
& Z_{W C T}=R_{T E}\left(\frac{j \omega}{\omega_{T E}}\right)^{-0.5} \tanh \left[\left(\frac{j \omega}{\omega_{T E}}\right)^{0.5}\right], \\
& Z_{W a}=R_{C}\left(\frac{j \omega}{\omega_{C}}\right)^{-0.5} \tanh \left[\left(\frac{j \omega}{\omega_{C}}\right)^{0.5}\right]
\end{aligned}
$$

where $\rho_{T E}, L$, and $A$ are the electrical resistivity, length, and cross-sectional area of the TE material, respectively, $j^{2}=-1, \omega$ is the angular frequency, and $\omega_{T E}$ and $\omega_{C}$ are the characteristic angular frequencies of thermal diffusion in the TE sample $\left[\omega_{T E}=\alpha_{T E} /(L / 2)^{2} ; \alpha_{T E}\right.$ denoting the thermal diffusivity of the TE material] and in the contact $\left(\omega_{C}=\alpha_{C} / L_{C}{ }^{2} ; \alpha_{C}\right.$ denoting the thermal diffusivity of the contact). $R_{T E}$ is the TE resistance [27], and $R_{C}$ is a TE resistance induced by the contact. They are given by,

$$
\begin{aligned}
& R_{T E}=\frac{S^{2} T_{i} L}{\lambda_{T E} A}, \\
& R_{C}=2 \frac{S^{2} T_{i} L_{C}}{\lambda_{C} A},
\end{aligned}
$$

where $\lambda_{T E}$ and $\lambda_{C}$ are the thermal conductivity of the TE material and the stainless-steel contact, respectively, $T_{i}$ is the absolute ambient temperature, and $L_{C}$ the length of the latter. 
From the curve fits, $R_{\Omega}, R_{T E}, R_{C}, \omega_{T E}$, and $\omega_{C}$ can be obtained. Hence, using Eq. (2.1), the electrical resistivity can be determined as,

$$
\rho_{T E}=\frac{R_{\Omega} A}{L} .
$$

From Eq. (4.5), the Seebeck coefficient can be obtained as,

$$
S=\sqrt{\frac{R_{C} \lambda_{C} A}{2 T L_{C}}} .
$$

It should be noted that in order to determine $S$ in Eq. (7), the thermal conductivity of the stainless steel contact is required, for which literature values may be used [28]. From Eq. (3.4),

$$
\lambda_{T E}=\frac{S^{2} T_{i} L}{R_{T E} A} .
$$

Finally, combining Eq. (2.1) and Eq. (3.4),

$$
z T=\frac{R_{T E}}{R_{\Omega}} .
$$

\subsubsection{Characterization by the impedance method}

Five cycles were performed on the skutterudite sample, each cycle comprising a set of five impedance measurements at different temperatures $\left(50,100,150,200\right.$ and $\left.250{ }^{\circ} \mathrm{C}\right)$. Before the beginning of each cycle, the sample was newly assembled with fresh contacts. In order to obtain accurate impedance results, it is important to establish a suitable current amplitude for the measurements (the lowest amplitude possible with non-noisy measurements). This is to minimize the influence of non-linear effects such as the Joule heating and the variation of the TE properties with temperature, as discussed in our previous papers [25,29]. Hence, before performing the cycles, IS measurements at different current amplitudes (40, 60, 80, 100 and $120 \mathrm{~mA})$ were performed at each temperature in order to identify their optimal values. Fig. 4.3 shows the experimental impedance spectra and the corresponding fits for one of the five cycles measured. Fitting errors below $0.1,0.5$, and $12 \%$ were obtained for $R_{\Omega}, R_{T E}$, and $R_{C}$, respectively. It can be observed that even for the spectrum at $50{ }^{\circ} \mathrm{C}$, where the skutterudite shows lower performance and the impedance values are very small, the impedance response is clearly observed. In any case, the measured points differ in just few tenths of $\mu \Omega$ at high frequencies (bottom left part), and a 
powerful impedance analyzer (with high resolution and accuracy) is required in order to obtain sensitive measurements.

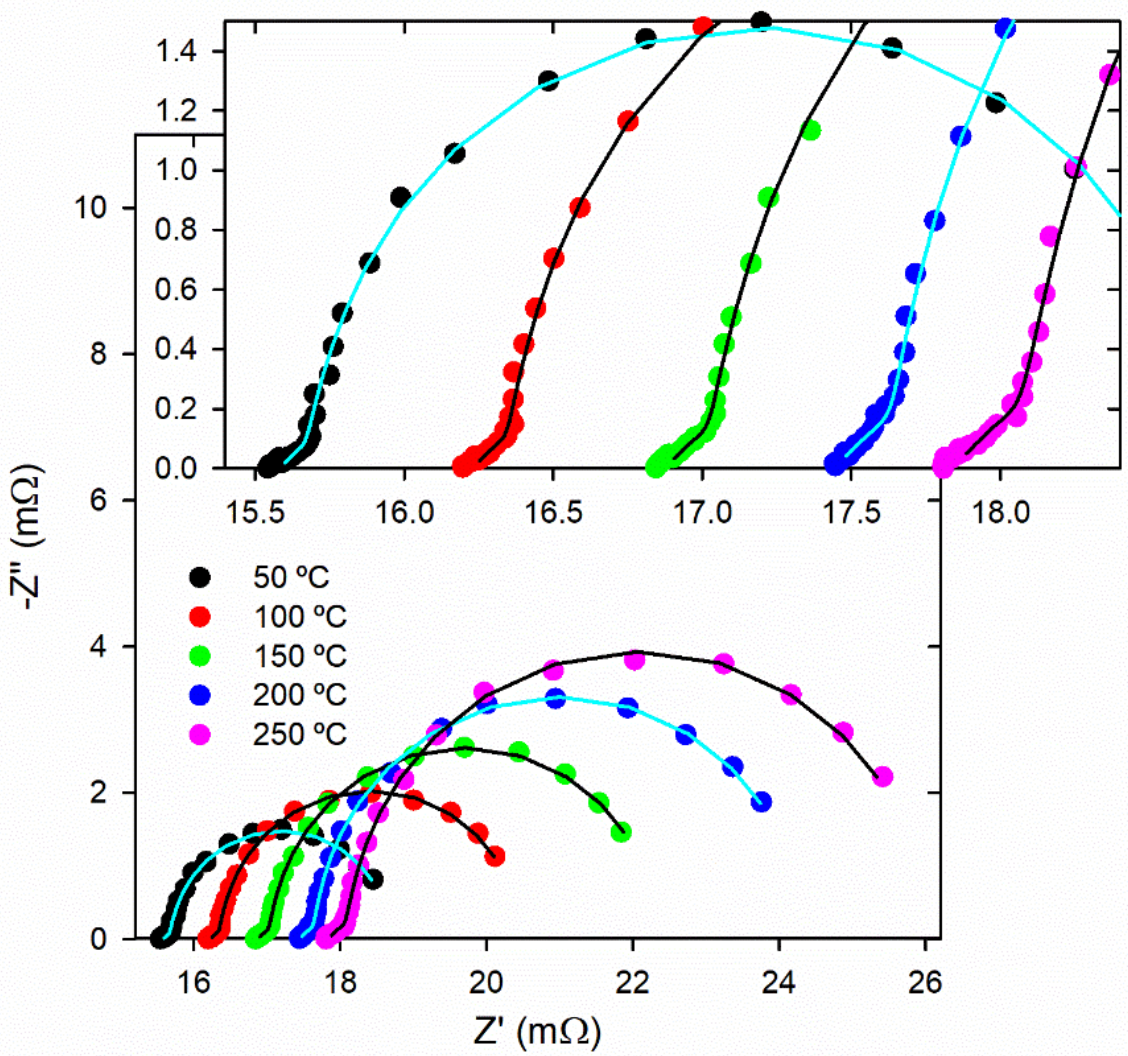

Fig. 4.3. Impedance spectroscopy measurements at different temperatures from one of the five measurement cycles performed. The dots represent the experimental values and the lines represent the fit to these data. The inset shows the magnification of the high frequency part.

The TE properties were obtained from Eq. (3.6) to Eq. (3.8) using the average values of $R_{\Omega}$, $R_{T E}$, and $R_{C}$ from the five measurements at each temperature. The thermal conductivity of the contact $\lambda_{C}$ (stainless steel AISI 304), which was needed for the Seebeck coefficient determination, was obtained from [28],

$$
\lambda_{C}=10.33+15.4 \times 10^{-3} T-7.0 \times 10^{-7} T^{2} .
$$

The validity of Eq. (4.10) was verified by performing measurements of the stainless steel AISI 304 thermal conductivity by a laser flash apparatus (LFA 467 HT from Netzsch) up to $150{ }^{\circ} \mathrm{C}$. The deviations found with respect to the equation were lower than $2.7 \%$. It is important in order 
to clearly discern the $45^{\circ}$ straight line feature at high frequencies (see bottom part of the inset of Fig. 4.3) that $\lambda_{C}$ is around an order of magnitude higher than $\lambda_{T E}$, otherwise this feature will overlap with the semicircle part.
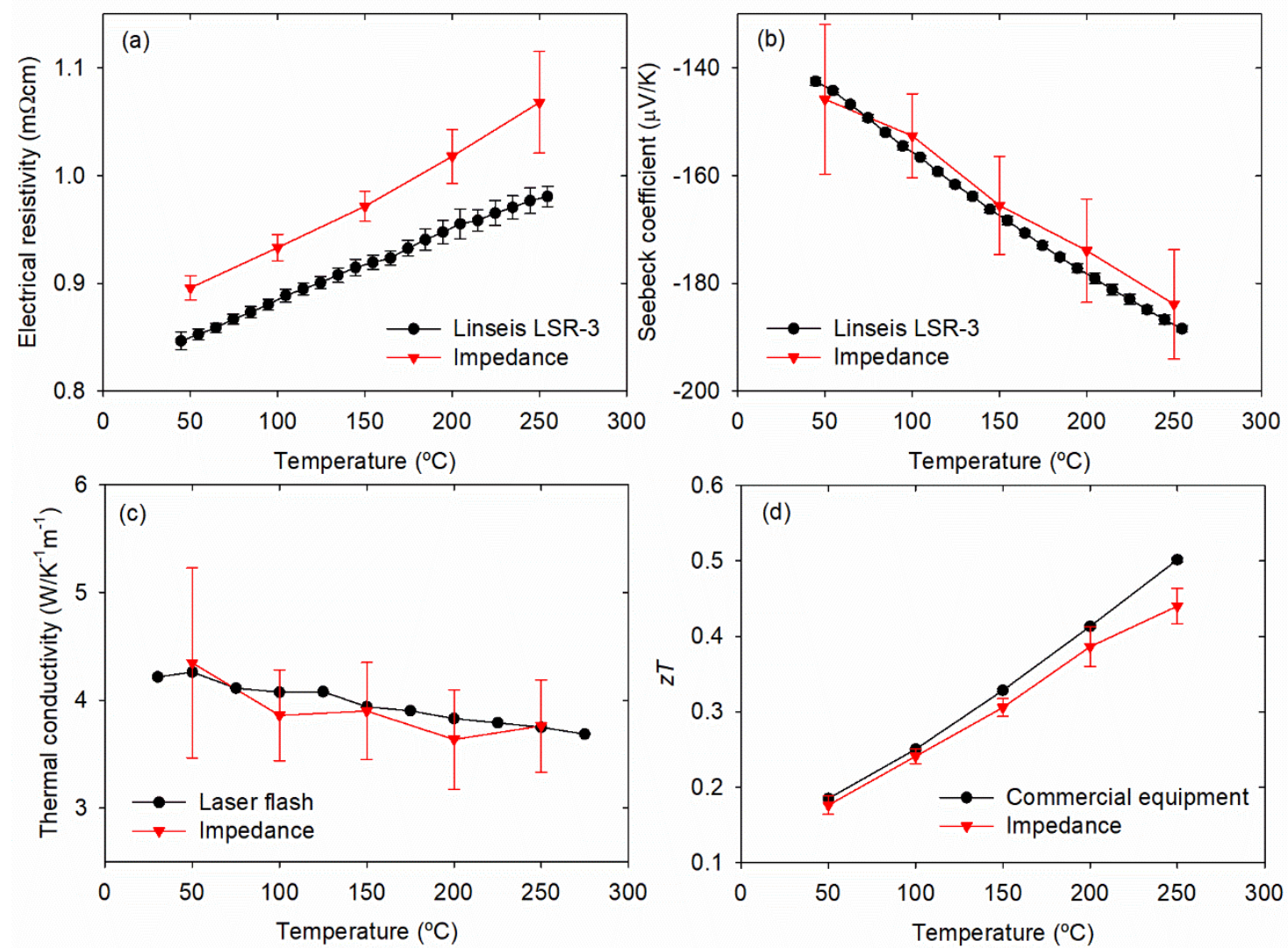

Fig. 4.4. (a) Electrical resistivity, (b) Seebeck coefficient, (c) thermal conductivity, and (d) $z T$ values extracted from the impedance method and compared with results from different commercial equipment. The error bars account for the total combined random errors $\left(u_{c}\right)$, excluding the contribution from the specific heat for the laser flash case. The confidence interval is $1 \sigma$.

Fig. 4.4 shows the TE properties obtained by the IS method compared with results from commercial equipment. All the properties show a good agreement with the commercial equipment measurements, except the electrical resistivity [Fig. 4.4(a)], which shows slightly higher values (around 6\%), which is due to the contribution from the contact resistance, which is not completely suppressed since the $\mathrm{W}$ wires are inserted at the junctions. It is known that Ga-In-Sn liquid metal can have nm-length native oxide layers at its surface, which can impact its wetting behavior and electrical resistivity. This might contribute to the higher electrical resistivity values found [30]. It 
can be also observed for this property that as the temperature increases the error bars become larger and the trend slightly deviates from the behavior found with the commercial equipment. This is related to the fact that the Ga-In-Sn liquid metal tends to react with the TE sample at around $250{ }^{\circ} \mathrm{C}$. This is the limiting constraint on the maximum temperature of operation, since the rest of the elements of the setup can stand far higher temperature values. Hence, if a suitable solder or liquid metal for the sample to be measured were found, this method could increase its capability at higher temperatures. These aspects mentioned for the electrical resistivity also influence the $z T$ [Fig. 4.4(d)] due to Eq. (3.8), which exhibits larger errors and deviations at the highest temperatures.

\subsubsection{Precision and accuracy evaluation}

In order to quantify the precision and accuracy of the impedance method, random and systematic errors, respectively, were calculated for all the determined TE properties. The total combined random errors $u_{c}$ of each property were obtained using [31],

$$
u_{c}^{2}=\sum_{i=1}^{N}\left(\frac{\partial f_{p}}{\partial x_{i}}\right)^{2} u^{2}\left(x_{i}\right)
$$

being $f_{p}$ each of the TE properties $\left(S, \lambda_{T E}, \rho_{T E}\right.$, or $\left.z T\right)$, and $x_{i}$ each of the parameters with an associated error $u$. The random errors for the Seebeck coefficient were calculated taking into account (i) the standard deviation of the five measurements at each temperature to obtain the average value of $R_{C}$, (ii) the mean fitting error of the five $R_{C}$ measurements at each temperature, (iii) the uncertainty in the area of the sample, (iv) the uncertainty of the thermocouple $[u(T)=1$ ${ }^{\circ} \mathrm{C}$, and (v) the uncertainty in the length of the contacts, which was measured using a caliper $\left[u\left(L_{C}\right)=0.005 \mathrm{~mm}\right]$. The contribution of the thermal conductivity of the contact was neglected. From all the above contributions, (i) and (ii) were the most significant compared to the others, which can be considered negligible.

The random errors for the thermal conductivity were calculated taking into account (i) the uncertainty of the Seebeck coefficient $\left[u_{c}(S)\right]$, (ii) the uncertainty of the thermocouple $[u(T)=1$ $\left.{ }^{\circ} \mathrm{C}\right]$, (iii) the uncertainty in the length of the sample $[u(L)=0.005 \mathrm{~mm}$, (iv) the uncertainty in the area of the sample, and (v) the standard deviation of the five measurements at each temperature to obtain the average value of $R_{T E}$. The contribution from the fitting errors in $R_{T E}$ (which were 
$<0.5 \%$ ) was discarded since it was negligible in comparison with the standard deviation. From all the contributions considered, the uncertainty in the Seebeck coefficient and the standard deviation of $R_{T E}$ are the most significant, the Seebeck contribution being an order of magnitude higher. Hence, the precision in the thermal conductivity determination is strongly influenced by the precision in the Seebeck coefficient measurement.

The random errors for the electrical resistivity were calculated taking into account (i) the uncertainty in the length of the sample, (ii) the uncertainty in the cross-sectional area, and (iii) the standard deviation from the five measurements at each temperature to obtain the average $R_{\Omega}$. It should be noticed that the latter contribution is the most significant, since it is around two orders of magnitude larger than the others. As occurred for $R_{T E}$, the contribution of the fitting errors for $R_{\Omega}(<0.1 \%)$ was neglected. Finally, the random errors for $z T$ were calculated from the contributions of the standard deviations of both $R_{\Omega}$ and $R_{T E}$. The error bars shown in Fig. 4.4 correspond to the calculated random errors for each property, which are also shown in Table 4.1. Most of the random errors are $\approx 5.5 \%,<13 \%,<2.5 \%$, and between 4 and $7 \%$ for $S, \lambda_{T E}, \rho_{T E}$, and $z T$, respectively, which demonstrates the good precision of the method, although the thermal conductivity is less precise due to the quadratic dependence on the Seebeck coefficient [see Eq. (3.7)]. At $50^{\circ} \mathrm{C}$ higher values are found for $S$ and $\lambda_{T E}$ due to a lower degree of repeatability at this temperature in one of the 5 cycles performed.

Systematic errors $u_{s}$ were calculated for the TE properties considering as true values the results obtained from the commercial equipment. They are also included in Table 4.1. Systematic errors are $<2.5 \%,<5.5 \%$, between 5 and $9 \%$, and $<9 \%$ for the $S, \lambda_{T E}, \rho_{T E}$, and $z T$, respectively, demonstrating a good agreement with the characterization performed with commercial equipment.

Finally, the total uncertainty of the method $u_{T}$ is obtained for each property as $u_{T}=\left(u_{c}^{2}+u_{S}^{2}\right)^{0.5}$ and also shown in Table 4.1. For $S$ and $\lambda_{T E}$ the total errors are predominantly $<6 \%$ and $<14 \%$, respectively. For these two parameters, it is evident that the principal contribution to the total error comes from the random error, which is higher than the systematic contribution. For $\rho_{T E}$ and $z T$, total errors are approximately from 5 to $10 \%$, and from 4 to $10 \%$, respectively. In this case the 
random and systematic contributions do not show the large differences as in the case of $S$ and $\lambda_{T E}$ and more equally contribute to the total error.

Table 4.1. Average values with their associated random, systematic and total errors of the thermoelectric properties of the skutterudite sample obtained by the impedance spectroscopy method.

\begin{tabular}{|c|c|c|c|c|c|}
\hline & $\begin{array}{c}\text { Temperature } \\
\left({ }^{\circ} \mathrm{C}\right)\end{array}$ & Mean value & $\begin{array}{c}\text { Systematic } \\
\text { error }(\%)\end{array}$ & $\begin{array}{l}\text { Random } \\
\text { error }(\%)\end{array}$ & $\begin{array}{c}\text { Total error } \\
(\%)\end{array}$ \\
\hline \multirow{5}{*}{$\begin{array}{c}\text { Seebeck } \\
\text { coefficient }(S)\end{array}$} & 50 & $-145.8 \mu \mathrm{VK}^{-1}$ & 1.08 & 9.51 & 9.57 \\
\hline & 100 & $-152.6 \mu \mathrm{VK}^{-1}$ & 1.89 & 5.11 & 5.45 \\
\hline & 150 & $-165.5 \mu \mathrm{VK}^{-1}$ & 1.13 & 5.48 & 5.60 \\
\hline & 200 & $-173.9 \mu \mathrm{VK}^{-1}$ & 2.34 & 5.51 & 5.99 \\
\hline & 250 & $-183.9 \mu \mathrm{VK}^{-1}$ & 2.03 & 5.51 & 5.87 \\
\hline \multirow{5}{*}{$\begin{array}{c}\text { Thermal } \\
\text { conductivity } \\
(\lambda T E)\end{array}$} & 50 & $4.35 \mathrm{WK}^{-1} \mathrm{~m}^{-1}$ & 2.00 & 20.26 & 20.36 \\
\hline & 100 & $3.86 \mathrm{WK}^{-1} \mathrm{~m}^{-1}$ & 5.28 & 10.93 & 12.14 \\
\hline & 150 & $3.90 \mathrm{WK}^{-1} \mathrm{~m}^{-1}$ & 0.99 & 11.54 & 11.58 \\
\hline & 200 & $3.64 \mathrm{WK}^{-1} \mathrm{~m}^{-1}$ & 5.05 & 12.71 & 13.68 \\
\hline & 250 & $3.76 \mathrm{WK}^{-1} \mathrm{~m}^{-1}$ & 0.34 & 11.40 & 11.41 \\
\hline \multirow{5}{*}{$\begin{array}{l}\text { Electrical } \\
\text { resistivity } \\
\quad\left(\rho_{T E}\right)\end{array}$} & 50 & $0.896 \mathrm{~m} \Omega \mathrm{cm}$ & 5.06 & 1.24 & 5.21 \\
\hline & 100 & $0.933 \mathrm{~m} \Omega \mathrm{cm}$ & 5.53 & 1.31 & 5.68 \\
\hline & 150 & $0.972 \mathrm{~m} \Omega \mathrm{cm}$ & 6.07 & 1.43 & 6.24 \\
\hline & 200 & $1.018 \mathrm{~m} \Omega \mathrm{cm}$ & 7.43 & 2.47 & 7.83 \\
\hline & 250 & $1.068 \mathrm{~m} \Omega \mathrm{cm}$ & 9.06 & 4.41 & 10.08 \\
\hline \multirow{5}{*}{$\begin{array}{c}\text { Dimensionless } \\
\text { figure of } \\
\text { merit }(z T)\end{array}$} & 50 & 0.176 & 6.69 & 7.07 & 9.73 \\
\hline & 100 & 0.241 & 0.04 & 4.07 & 4.07 \\
\hline & 150 & 0.306 & 4.78 & 3.85 & 6.13 \\
\hline & 200 & 0.386 & 1.97 & 6.77 & 7.05 \\
\hline & 250 & 0.440 & 8.62 & 5.29 & 10.12 \\
\hline
\end{tabular}

\subsection{Conclusions}

In summary, the ability to perform a complete characterization of all TE properties of a bulk material as a function of temperature, from a single electrical IS measurement, using one apparatus is demonstrated for a low-performance TE material (skutterudite sample) of modest properties up to $250{ }^{\circ} \mathrm{C}$. The TE properties were determined from fittings performed to the experimental impedance spectra employing a suitable equivalent circuit. Random errors were 
calculated by performing five measurements at each temperature remaking contacts, showing a good precision of the method $\left(\approx 5.5 \%,<13 \%,<2.5 \%\right.$, and between 4 and $7 \%$ for the $S, \lambda_{T E}, \rho_{T E}$, and $z T$, respectively). The random errors in the determination of thermal conductivity are higher due to the quadratic dependence of this property with the Seebeck coefficient. Systematic errors were also calculated by comparison with characterization results from commercial equipment obtained from the same sample, resulting in errors $<2.5 \%,<5.5 \%$, between 5 and $9 \%$, and $<9 \%$ for the $S, \lambda_{T E}, \rho_{T E}$, and $z T$, respectively, which illustrates the accuracy of the method. These results demonstrate the potential of the method as a powerful tool to significantly facilitate the task of characterization of bulk TE materials and thus the search for a more efficient heat to electricity energy conversion.

\section{Acknowledgements}

BBP and JGC acknowledge financial support from the Spanish Agencia Estatal de Investigación under the Ramón y Cajal program (RYC-2013-13970), from the Generalitat Valenciana and the European Social Fund under the ACIF program (ACIF/2018/233), from the Universitat Jaume I under the project UJI-A2016-08, and the technical support of Raquel Oliver Valls and José Ortega Herreros. AVP and JPG wish to thank the UK Engineering and Physical Sciences Research Council (EP/K019767/1) for financial support.

\section{References}

[1] Z. Fan, J.C. Ho, B. Huang, Chapter 11. One-Dimensional Nanostructures for Energy Harvesting, in: One-Dimensional Nanostructures Princ. Appl., John Wiley \& Sons, Inc., Hoboken, NJ, USA, 2013: pp. 237-270. https://doi.org/10.1002/9781118310342.ch11.

[2] R. Patowary, D.C. Baruah, Thermoelectric conversion of waste heat from IC enginedriven vehicles: A review of its application, issues, and solutions, Int. J. Energy Res. 42 (2018) 2595-2614. https://doi.org/10.1002/er.4021.

[3] D. Champier, Thermoelectric generators: A review of applications, Energy Convers. Manag. 140 (2017) 167-181. https://doi.org/10.1016/j.enconman.2017.02.070.

[4] A.R.M. Siddique, S. Mahmud, B. Van Heyst, A review of the state of the science on 
wearable thermoelectric power generators (TEGs) and their existing challenges, Renew. Sustain. Energy Rev. (2017). https://doi.org/10.1016/j.rser.2017.01.177.

[5] G.J. Snyder, E.S. Toberer, Complex thermoelectric materials., Nat. Mater. 7 (2008) 105114.

[6] K.A. Borup, J. de Boor, H. Wang, F. Drymiotis, F. Gascoin, X. Shi, L. Chen, M.I. Fedorov, E. Müller, B.B. Iversen, G.J. Snyder, Measuring thermoelectric transport properties of materials, 8 (2015) 423-435. https://doi.org/10.1039/C4EE01320D.

[7] U. Stöhrer, Measurement of the transport properties of FeSi2 and HMS by utilization of the Peltier effect in the temperature range $50-800{ }^{\circ} \mathrm{C}$, Meas. Sci. Technol. 5 (1994) 440446.

[8] J. de Boor, V. Schmidt, Complete Characterization of Thermoelectric Materials by a Combined van der Pauw Approach, Adv. Mater. 22 (2010) 4303. https://doi.org/10.1002/adma.201001654.

[9] R.L. Kallaher, C.A. Latham, F. Sharifi, An apparatus for concurrent measurement of thermoelectric material parameters, Rev. Sci. Instrum. $84 \quad$ (2013) 013907. https://doi.org/10.1063/1.4789311.

[10] H. Kolb, T. Dasgupta, K. Zabrocki, E. Mueller, J. De Boor, Simultaneous measurement of all thermoelectric properties of bulk materials in the temperature range $300-600 \mathrm{~K}$, Rev. Sci. Instrum. 86 (2015) 073901. https://doi.org/10.1063/1.4926404.

[11] D. Vasilevskiy, J.M. Simard, R.A. Masut, S. Turenne, System for Simultaneous HarmanBased Measurement of All Thermoelectric Properties, from 240 to $720 \mathrm{~K}$, by Use of a Novel Calibration Procedure, J. Electron. Mater. 44 (2015) 1733-1742. https://doi.org/10.1007/s11664-014-3531-5.

[12] J. Martin, W. Wong-Ng, M.L. Green, Seebeck Coefficient Metrology: Do Contemporary Protocols Measure Up?, J. Electron. Mater. 44 (2015) 1998-2006. https://doi.org/10.1007/s11664-015-3640-9.

[13] S. Dilhaire, L.D. Patino-Lopez, S. Grauby, J.M. Rampnoux, S. Jorez, W. Claeys, Determination of ZT of PN thermoelectric couples by AC electrical measurement, in: Int. 
Conf. Thermoelectr. ICT, Proc., Institute of Electrical and Electronics Engineers Inc., 2002: pp. 321-324. https://doi.org/10.1109/ICT.2002.1190330.

[14] A.D. Downey, T.P. Hogan, B. Cook, Characterization of thermoelectric elements and devices by impedance spectroscopy, Rev. Sci. Instrum. 78 (2007) 093904. https://doi.org/10.1063/1.2775432.

[15] J. García-cañadas, G. Min, Impedance spectroscopy models for the complete characterization of thermoelectric materials, J. Appl. Phys. 116 (2014). https://doi.org/10.1063/1.4901213.

[16] C.Y. Yoo, Y. Kim, J. Hwang, H. Yoon, B.J. Cho, G. Min, S.H. Park, Impedance spectroscopy for assessment of thermoelectric module properties under a practical $\begin{array}{lllll}\text { operating } & \text { temperature, } & \text { Energy. } & 152 & \text { (2018) }\end{array}$ https://doi.org/10.1016/j.energy.2017.12.014.

[17] R. Mesalam, H.R. Williams, R.M. Ambrosi, J. García-Cañadas, K. Stephenson, Towards a comprehensive model for characterising and assessing thermoelectric modules by impedance spectroscopy, Appl. Energy. https://doi.org/10.1016/j.apenergy.2018.05.041.

[18] E. Thiébaut, F. Pesty, C. Goupil, G. Guegan, P. Lecoeur, Non-linear impedance spectroscopy for complete thermoelectric characterization: Beyond the zT estimation, J. Appl. Phys. 124 (2018) 235106. https://doi.org/10.1063/1.5063419.

[19] F. Fabregat-Santiago, G. Garcia-Belmonte, I. Mora-Seró, J. Bisquert, Characterization of nanostructured hybrid and organic solar cells by impedance spectroscopy, Phys. Chem. Chem. Phys. 13 (2011). https://doi.org/10.1039/c0cp02249g.

[20] I. Mora-Sero, G.A. Garcia-Belmonte, P.P. Boix, M.A. Vazquez, J. Bisquert, Impedance spectroscopy characterisation of highly efficient silicon solar cells under different light illumination intensities, Energy Environ. Sci. 2 (2009) 678-686. https://doi.org/10.1039/b812468j.

[21] X. Yuan, H. Wang, J. Colinsun, J. Zhang, AC impedance technique in PEM fuel cell diagnosis? A review, Int. J. Hydrogen Energy. 32 (2007) 4365-4380. 
[22] R. Kötz, M. Hahn, R. Gallay, Temperature behavior and impedance fundamentals of supercapacitors, J. Power Sources. $154 \quad$ (2006) 550-555. https://doi.org/10.1016/J.JPOWSOUR.2005.10.048.

[23] G.W. Walter, A review of impedance plot methods used for corrosion performance analysis of painted metals, Corros. Sci. 26 (1986) 681-703. https://doi.org/10.1016/0010938X(86)90033-8.

[24] J.T.S. Irvine, D.C. Sinclair, A.R. West, Electroceramics: Characterization by Impedance Spectroscopy, Adv. $\quad$ Mater. $2 \quad$ (1990) 132-138. https://doi.org/10.1002/adma.19900020304.

[25] B. Beltrán-Pitarch, J. Prado-Gonjal, A. V. Powell, P. Ziolkowski, J. García-Cañadas, Thermal conductivity, electrical resistivity, and dimensionless figure of merit (ZT) determination of thermoelectric materials by impedance spectroscopy up to $250{ }^{\circ} \mathrm{C}$, J. Appl. Phys. 124 (2018) 025105. https://doi.org/10.1063/1.5036937.

[26] J. Prado-Gonjal, M. Phillips, P. Vaqueiro, G. Min, A. V. Powell, Skutterudite Thermoelectric Modules with High Volume-Power-Density: Scalability and Reproducibility, ACS Appl. Energy Mater. 1 (2018) 6609-6618. https://doi.org/10.1021/acsaem.8b01548.

[27] J. García-Cañadas, G. Min, Low frequency impedance spectroscopy analysis of thermoelectric modules, J. Electron. Mater. 43 (2014) 2411-2414. https://doi.org/10.1007/s11664-014-3095-4.

[28] J.J. Valencia, P.N. Quested, Thermophysical Properties, 2008. https://doi.org/10.1361/asmhba0005240.

[29] B. Beltrán-Pitarch, J. Prado-Gonjal, A. V. Powell, J. García-Cañadas, Experimental conditions required for accurate measurements of electrical resistivity, thermal conductivity, and dimensionless figure of merit (ZT) using Harman and impedance spectroscopy methods, J. Appl. Phys. (2019). https://doi.org/10.1063/1.5077071.

[30] M.A.H. Khondoker, D. Sameoto, Fabrication methods and applications of microstructured gallium based liquid metal alloys, Smart Mater. Struct. 25 (2016) 093001. 


\section{Chapter 4}

https://doi.org/10.1088/0964-1726/25/9/093001.

[31] Evaluation of measurement data-Guide to the expression of uncertainty in measurement, (1995). https://www.bipm.org/en/publications/guides/gum.html (accessed October 23, 2018). 
5. Influence of convection at outer ceramic surfaces on the characterization of thermoelectric modules by impedance spectroscopy 


\title{
Influence of convection on the characterization of thermoelectric modules by impedance spectroscopy
}

\author{
Braulio Beltrán-Pitarch, Jorge García-Cañadas*
}

Department of Industrial Systems Engineering and Design, Universitat Jaume I, Campus del Riu Sec, 12071 Castellón, Spain

*e-mail: garciaj@uji.es 


\begin{abstract}
Impedance spectroscopy (IS) is a useful method for the characterization of thermoelectric (TE) modules. It can determine with high accuracy the module dimensionless figure of merit $\left(Z_{m} T\right)$ as well as the average TE properties of the module's thermoelements. Interpretation of impedance results require the use of a theoretical model (equivalent circuit) which provides the desired device parameters after a fitting is performed to the experimental results. Here we extend the currently available equivalent circuit, only valid for adiabatic conditions, to account for the effect of convection at the outer surface of the module ceramic plates, which is the part of the device where convection is more prominent. This is performed by solving the heat equation in the frequency domain including convection heat losses. As a result, a new element (convection resistance) appears in the developed equivalent circuit, which starts to influence at mid-low frequencies, causing a decrease of the typically observed semicircle in the impedance spectrum. If this effect is not taken into account, an underestimation of the $Z_{m} T$ occurs when measurements under room conditions are performed. The theoretical model is validated by experimental measurements performed in a commercial module with and without vacuum. Interestingly, the use of the new equivalent circuit allows the determination of the convection heat transfer coefficient $(h)$ if the module's Seebeck coefficient is known and an impedance measurement in vacuum is performed, opening up the possibility to develop TE modules as $h$ sensors. On the other hand, if $h$ is known, all the properties of the module $\left(Z_{m} T\right.$, ohmic (internal) resistance, average Seebeck coefficient and average thermal conductivity of the thermoelements, and thermal conductivity of the ceramics) can be obtained from one impedance measurement in vacuum and another measurement at room conditions.
\end{abstract}




\section{Graphical abstract}
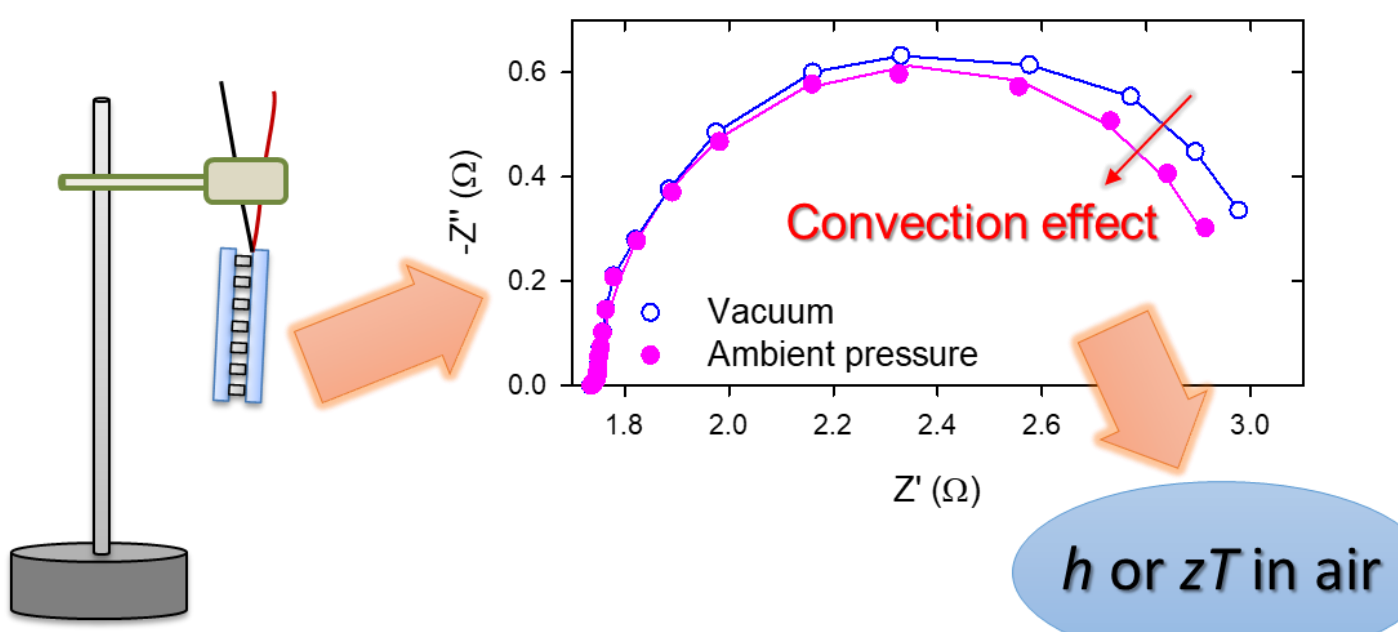

Fig. 5.1. Graphical abstract of the work: Influence of convection at outer ceramic surfaces on the characterization of thermoelectric modules by impedance spectroscopy. 


\subsection{Introduction}

Impedance spectroscopy (IS) has been proved to be an accurate and quick method to measure the dimensionless figure of merit of thermoelectric (TE) modules [1-7]. In addition, it also allows a complete characterization of these devices if the thermal conductivity of the ceramics is given, providing the ohmic (internal) resistance, the module $Z_{m} T$, and the average thermoelements Seebeck coefficient and thermal conductivity $[6,8,9]$. Interpretation of impedance results typically require the use of a theoretical model (equivalent circuit) which provides the desired device parameters after a fitting is performed to the experimental results. We have recently developed the equivalent circuit for suspended TE modules under adiabatic conditions, which consists of the ohmic module resistance connected in series with a parallel combination of two Warburg elements $[8,10]$. However, this equivalent circuit, since considers adiabatic conditions, does not take into account the effect of convection at the outer surface of the ceramic plates, which is the part of the device where convection is more prominent. This can significantly influence the impedance response when measurements are not performed in vacuum and provide an inaccurate module characterization [5].

In this work, we extend the previously reported equivalent circuit to include the effect of convection at the outer surface of the ceramics. This is achieved by solving the heat equation in the frequency domain with convective heat fluxes at the ceramic boundaries. The complete analysis provides three new elements which for standard commercial TE modules can be simplified to only one, a convection resistance, which is connected in parallel to the two Warburg elements. An experimental validation of the new equivalent circuit is performed with a commercial TE module, which is measured in vacuum and at room conditions. Finally, new possibilities of the developed equivalent circuit for module and convection characterization are discussed.

\subsection{Theoretical model}

In order to calculate the impedance function of the system a monodimensional model as shown in Fig. 5.2 is considered. This model consists of a TE material of cross-sectional area $A$ and length $L$ contacted by two ceramic contacts of similar area, simulating a TE leg inside a TE module. The thermal influence of the copper interconnects is neglected due to the high thermal conductivity of 
copper and their short length [2,8]. In addition, the model does not consider spreading-constriction effects of the heat flow due to the dissimilar areas between the TE elements and the ceramics [11]. On the other hand, due to the small ac amplitude used in the impedance measurements and the high electrical conductivity of the TE materials the Joule effect is neglected. Finally, the TE properties are considered independent on temperature $T$ and the system is considered adiabatic, except at the outer surfaces of the ceramics, where the convection influence is evaluated. It should be noted that this model only considers a single TE leg, so the final impedance response should be multiplied by the number of legs of the TE module ( $2 N$, being $N$ the number of couples).

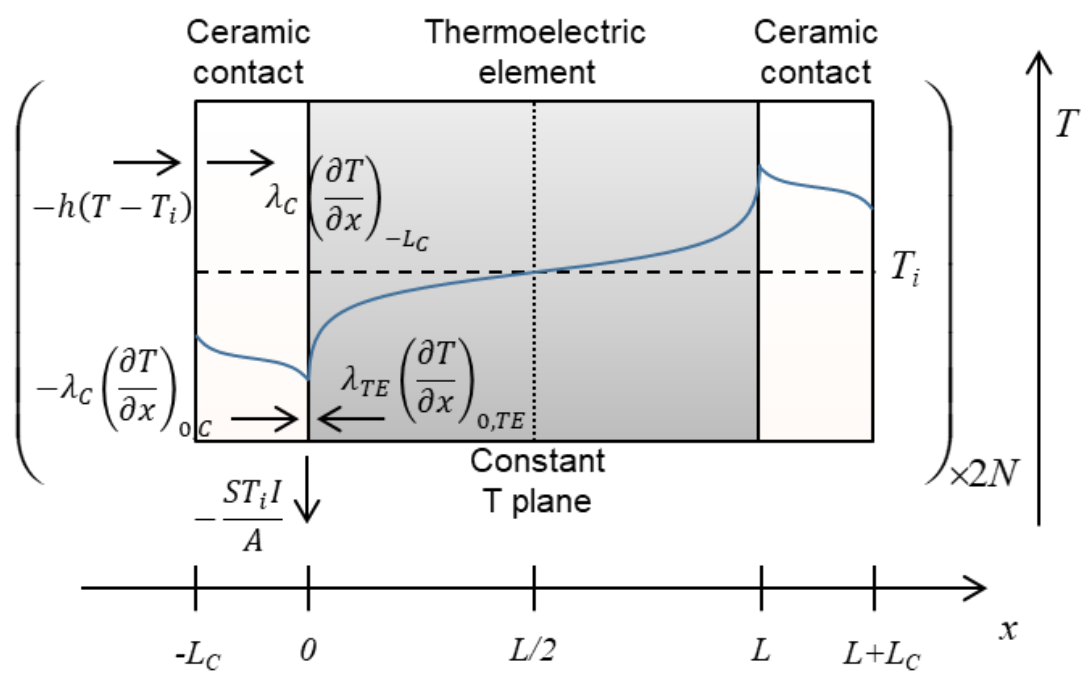

Fig. 5.2. Thermal model employed in the theoretical analysis. A positive value of both the current and the Seebeck coefficient is considered. The arrows indicate the direction of the heat fluxes appearing at the boundaries. For Peltier heat, the arrows point out of the junction when the electrons absorb heat from the lattice. The solid line qualitatively depicts a possible thermal profile. The dotted line shows the plane where the temperature remains constant at any time and the dashed line indicates the initial temperature.

The impedance function $Z=V / I$ of a TE module under the above considerations is given by,

$$
Z=\frac{V(0)-V(L)}{I_{0}}=R_{\Omega}+2 N \frac{|S|[T(L)-T(0)]}{I_{0}}=R_{\Omega}-2 N \frac{2|S|\left[T(0)-T_{i}\right]}{I_{0}},
$$

where $V(0)$ and $V(L)$ are the voltages at $x=0$ and $x=L$, respectively, $I_{0}$ is the electrical current flowing through the device at $x=0, R_{\Omega}$ is the total ohmic resistance, which includes the contribution of all the TE legs, the copper interconnects, the wires, and the contact resistances, $S$ is the average Seebeck coefficient of each thermoelement, and $T(0)$ and $T(L)$ are the temperatures at $x=0$ and $x=L$, respectively. It should be noted in Eq. (3.4) that due to the symmetry of the system 
with respect to the constant temperature plane (see Fig. 5.2), the temperature difference across the thermoelement can be determined from the temperature value at $x=0$.

To determine the variation with frequency of $T(0)$, the heat equation of the system in the frequency domain must be solved [8],

$$
\frac{\partial^{2} \theta}{\partial x^{2}}-\frac{j \omega}{\alpha_{i}} \theta=0
$$

where $\theta$ is the Laplace transform of the temperature with respect to the initial temperature $(\theta=L[T$ $\left.T_{i}\right]$ ), $j$ is the imaginary number, $\omega$ is the angular frequency (defined as $\omega=2 \pi f$, where $f$ is the frequency) and $\alpha_{i}$ is the thermal diffusivity of the TE leg $(i=T E)$ or the ceramic $(i=C)$.

The solution of Eq. (5.2) and its derivative is given by,

$$
\begin{aligned}
& \theta=C_{1, i} \sinh \left[\frac{x}{L_{i}}\left(\frac{j \omega}{\omega_{i}}\right)^{0.5}\right]+C_{2, i} \cosh \left[\frac{x}{L_{i}}\left(\frac{j \omega}{\omega_{i}}\right)^{0.5}\right], \\
& \frac{\partial \theta}{\partial x}=\frac{1}{L_{i}}\left(\frac{j \omega}{\omega_{i}}\right)^{0.5}\left\{C_{1, i} \cosh \left[\frac{x}{L_{i}}\left(\frac{j \omega}{\omega_{i}}\right)^{0.5}\right]+C_{2, i} \sinh \left[\frac{x}{L_{i}}\left(\frac{j \omega}{\omega_{i}}\right)^{0.5}\right]\right\},
\end{aligned}
$$

where $L_{i}$ is the half the length of the thermoelement $(i=T E)$ or the thickness of the ceramic contact ( $i=C$ ), $\omega_{i}$ is the characteristic angular frequency of each material (being $\omega_{i}=\alpha_{i} / L_{i}^{2}$ ), and $C_{l, i}$ and $C_{2, i}$ are constants.

From the thermal model in Fig. 5.2, four boundary conditions can be formulated,

$$
\begin{aligned}
& \theta(L / 2)=0, \text { at } x=L / 2, \\
& -\frac{S T_{i} i_{0}}{A}-\lambda_{C}\left(\frac{\partial \theta}{\partial x}\right)_{0, C}+\lambda_{T E}\left(\frac{\partial \theta}{\partial x}\right)_{0, T E}=0, \text { at } x=0, \\
& -\frac{h}{\eta} \theta\left(-L_{C}\right)+\lambda_{C}\left(\frac{\partial \theta}{\partial x}\right)_{-L_{C}}=0, \text { at } x=-L_{C}, \\
& \theta(0)_{T E}=\theta(0)_{C}, \text { at } x=0,
\end{aligned}
$$

where $i_{0}$ is the Laplace transform of the current at $x=0\left(i_{0}=\mathscr{L}\left[I_{0}\right]\right), h$ the convection heat transfer coefficient, $\eta$ the TE module filling factor, which is given by the ratio of the total area of the TE legs $(2 N A)$ to the total area of the ceramic plate, $\lambda_{C}$ the thermal conductivity of the ceramic, and $\lambda_{T E}$ the average thermal conductivity for each thermoelement. Eq. (5.5) defines the constant temperature at the half-length plane due to the symmetry of the system. Eq. (5.6) and Eq. (5.7) 
show the energy balance at $x=0$ and $x=-L_{C}$, respectively, where the convective heat flow is included in the latter. It should be noticed that the filling factor in this equation accounts for the convection produced in the area of the ceramic which differs from the area of the TE legs (2NA). In commercial modules $\eta$ can take values around 0.3 , which represents a significant part of the ceramic outer surface where convection occurs which should be taken into account. Finally, Eq. (5.8) shows the temperature continuity at the contacts,

Using these boundary conditions and Eq. (5.3) and Eq. (5.4), $\theta(0)$ can be determined,

$$
\begin{aligned}
\theta(0)=\frac{-S T_{i} i_{0}}{A}\left\{\frac{\lambda_{T E}}{(L / 2)}\left(\frac{j \omega}{\omega_{T E}}\right)^{0.5} \operatorname{coth}\left[\left(\frac{j \omega}{\omega_{T E}}\right)^{0.5}\right]\right. \\
\left.+\frac{\frac{\lambda_{C}}{L_{C}}\left(\frac{j \omega}{\omega_{C}}\right)^{0.5} \cosh \left[\left(\frac{j \omega}{\omega_{C}}\right)^{0.5}\right]+\frac{\lambda_{C}^{2}}{h L_{C}^{2}}\left(\frac{j \omega}{\omega_{C}}\right)^{0.5} \sinh \left[\left(\frac{j \omega}{\omega_{C}}\right)^{0.5}\right]}{\sinh \left[\left(\frac{j \omega}{\omega_{C}}\right)^{0.5}\right]+\frac{\lambda_{C}}{h L_{C}}\left(\frac{j \omega}{\omega_{C}}\right)^{0.5} \cosh \left[\left(\frac{j \omega}{\omega_{C}}\right)^{0.5}\right]}\right\}
\end{aligned}
$$

As shown in Eq. (3.4), the impedance function in the frequency domain can be obtained once $\theta(0)$ is known,

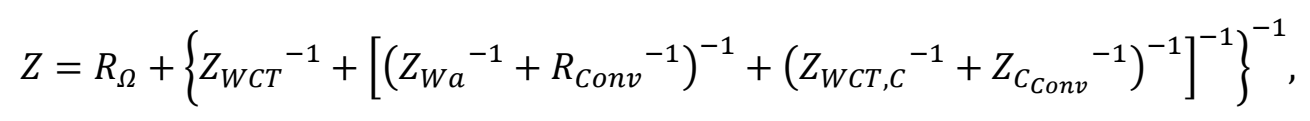

where the different elements in Eq. (5.10) are defined as,

$$
\begin{aligned}
& Z_{W C T}=\frac{2 N S^{2} T_{i} L}{\lambda_{T E} A}\left(\frac{j \omega}{\omega_{T E}}\right)^{-0.5} \tanh \left[\left(\frac{j \omega}{\omega_{T E}}\right)^{0.5}\right] \\
& Z_{W a}=\frac{4 N S^{2} T_{i} L_{C}}{\lambda_{C} A}\left(\frac{j \omega}{\omega_{C}}\right)^{-0.5} \operatorname{coth}\left[\left(\frac{j \omega}{\omega_{C}}\right)^{0.5}\right], \\
& R_{\text {conv }}=\frac{4 N S^{2} T_{i} \eta}{h A}, \\
& Z_{W C T, C}=\frac{4 N S^{2} T_{i} L_{C}}{\lambda_{C} A}\left(\frac{j \omega}{\omega_{C}}\right)^{-0.5} \tanh \left[\left(\frac{j \omega}{\omega_{C}}\right)^{0.5}\right], \\
& Z_{C_{\text {conv }}}=\frac{4 N S^{2} T_{i} L_{C}{ }^{2} h}{\lambda_{C}{ }^{2} A \eta}\left(\frac{j \omega}{\omega_{C}}\right)^{-1} .
\end{aligned}
$$

The equivalent circuit corresponding to Eq. (5.10) is show in Fig. 5.3(a). This equivalent circuit includes three new elements in addition to the $R_{\Omega}$, the constant-temperature Warburg 
$\left(Z_{W C T}\right)$ and the adiabatic Warburg $\left(Z_{W a}\right)$, which form the previously reported equivalent circuit which discarded convection effects [8,10]. The first new element, given in Eq. (5.13), is defined as a convection resistance $R_{\text {conv }}$ and can be represented by a resistor, since it takes a constant value and shows no dependency on frequency. It can be seen that $R_{\text {conv }}$ depends neither on the thermal properties of the thermoelectric material nor the ceramic, but it is influenced by the convection heat transfer coefficient. The physical meaning of $R_{\text {conv }}$ is related to the loss of heat energy from the ceramic produced by convection. These losses reduce the heat accumulation in this material and hence the temperature increase at the junction. The second element $Z_{W C T, C}$, defined in Eq. (5.14), takes the form of a constant-temperature Warburg [8] but with all its parameters corresponding to the ceramic material and multiplied by two, since two ceramic layers are present. The last new element $C_{\text {conv }}$, given in Eq. (5.15), shows the typical relationship with frequency of a capacitor $\left[\mathrm{Z}_{C c o n v}=\left(j \omega C_{\text {conv }}\right)^{-1}\right.$, being $\left.C_{\text {conv }}=\left(\lambda_{C}{ }^{2} A\right) /\left(4 N S^{2} T_{i} L_{C}{ }^{2} h \omega_{C}\right)\right]$ and it is defined as a convection capacitance. It should be noted that when the convection effect does not take place $(h=0) Z_{C c o n v}=0$, which creates a short circuit replacing the capacitor in Fig. 5.3(a), making

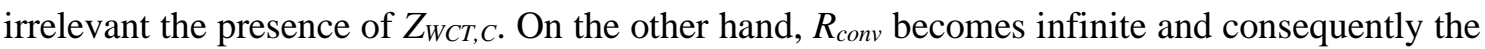
equivalent circuit in Fig. 5.3(a) reduces to the parallel combination of $Z_{W C T}$ and $Z_{W a}$ in series with $R_{\Omega}$, which is the previously reported equivalent circuit with no influence of convection [8].
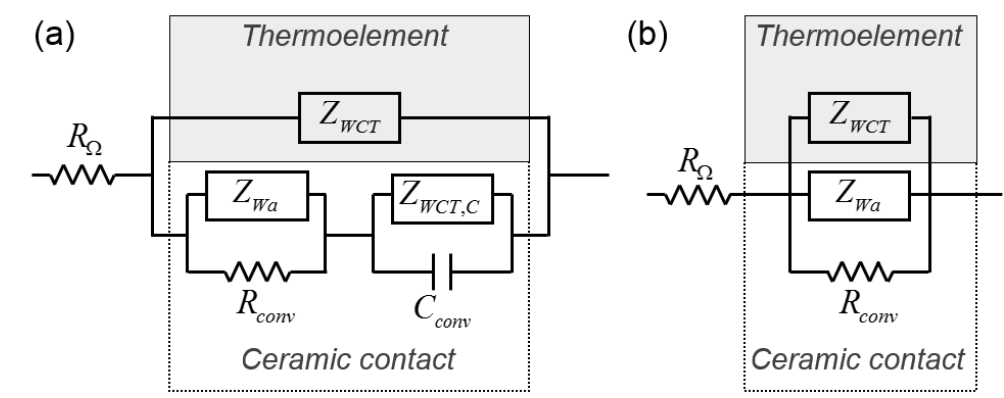

Fig. 5.3. (a) Equivalent circuit obtained when convection effects are considered at the outer surface of the ceramics in a thermoelectric module. (b) Simplified equivalent circuit for standard commercial thermoelectric modules. The equivalent circuit elements framed in the dotted line are related to the ceramic plates. The ones framed by the solid line in grey correspond to the thermoelement.

If the opposite case is considered, i.e. a huge convection $(h \rightarrow \infty)$ at the outer surface of the ceramics occurs, $R_{\text {conv }} \rightarrow 0$ and $Z_{C c o n v} \rightarrow \infty$, thus, the equivalent circuit in Fig. 5.3(a) reduces to the parallel combination of $Z_{W C T, C}$ with $Z_{W C T}$, connected in series with $R_{\Omega}$. In this case, the $Z_{W C T, C}$ element represents the diffusion of heat within the ceramic from the junction, which is completely 
removed by the effect of the convection when it reaches the outer surface of the ceramic, producing no temperature change at this surface (constant-temperature boundary) [10].

For the case of TE modules with ceramic plates $\left(\lambda_{c} \approx 35 \mathrm{WK}^{-1} \mathrm{~m}^{-1}\right)$ and conditions where the convection heat transfer coefficient is approximately $h<500 \mathrm{WK}^{-1} \mathrm{~m}^{-2}$, Eq. (5.10) can be simplified as,

$$
Z=R_{\Omega}+\left(Z_{W C T}{ }^{-1}+Z_{W a}^{-1}+R_{\text {conv }}{ }^{-1}\right)^{-1},
$$

since the impedance of the parallel combination of $Z_{W C T, C}$ and $C_{c o n v}$ is much lower than the impedance of the parallel combination of $Z_{W a}$ and $R_{\text {conv }}$. The equivalent circuit obtained from Eq. (5.16) can be seen in Fig. 5.3(b). In any case, it should be taken into account that this approximation is not valid if the convection heat transfer coefficient takes significantly higher values, or the TE module is formed by electrically insulating plates of thermal conductivity significantly lower than typical values of ceramics (e.g. polymers where $\lambda \approx 0.2 \mathrm{WK}^{-1} \mathrm{~m}^{-1}$ ), although this is not usually the case.

Some simulations of the equivalent circuit elements from Fig. 5.3(b) were performed to understand the effect of convection in the impedance spectra, which are shown in Fig. 5.4(a). As described in our previous article [10], $Z_{W a}$ would be obtained in the impedance spectrum in the hypothetical case where conduction of heat towards the TE elements is not produced $\left(\lambda_{T E}=0\right)$ and convection effects are neglected. In this case, all the Peltier heat diffuses from the junction towards the outer surface of the ceramic [slope-1 line from $Z_{W a}$ in the higher magnification inset of Fig. 5.4(a)]. Then, once the heat reaches the outer surface, it is accumulated in the ceramic, since it cannot escape due to the adiabatic conditions, producing the capacitive (vertical line) feature in the impedance response. However, when convection effects are considered, part of the heat reaching the outer surface can escape by convection, and the impedance response induced by the ceramic layers is now given by the parallel combination of $Z_{W a}$ and $R_{c o n v}$, which produces the closing of the vertical line at mid-low frequencies, as shown by the green line from Fig. 5.4(a). The effect of convection is not sensed until mid-low frequencies are reached [see Fig. 5.4(a)], since for the convection to occur it is required that the heat reaches the outer surface of the ceramics and also certain temperature increase in this material, which does not take place until mid-low frequencies. For this reason the $Z_{W a}$ element and the parallel combination of $Z_{W a}$ and 
$R_{\text {conv }}$ provide the same impedance response at mid-high frequencies [see the overlap in the insets of Fig. 5.4(a)].
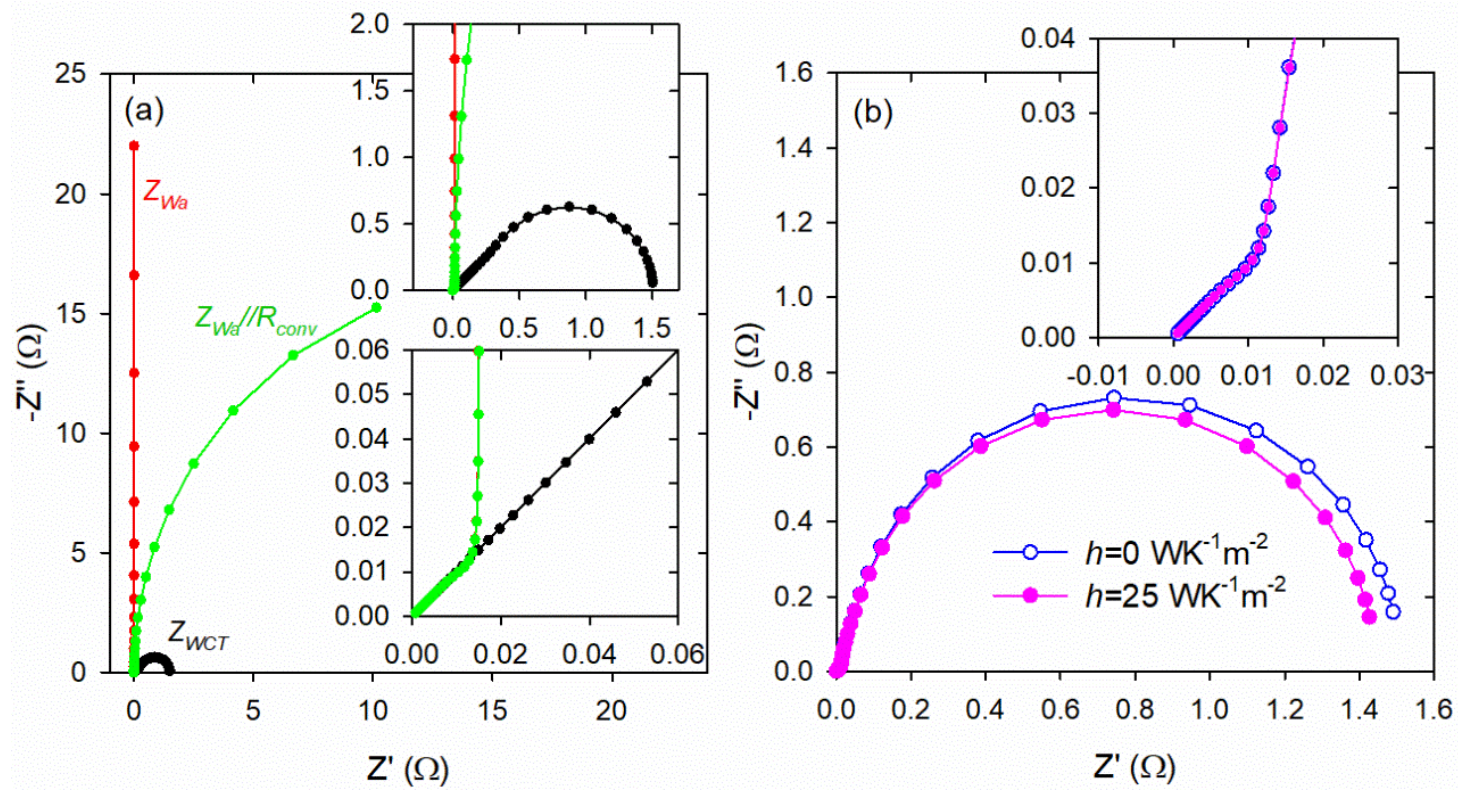

Fig. 5.4. (a) Impedance simulations in the $10 \mathrm{mHz}$ to $10 \mathrm{kHz}$ frequency range of the equivalent circuit elements $Z_{W a}$ (red), $Z_{W C T}$ (black) and the parallel combination of $Z_{W a}$ and $R_{c o n v}$ (green). The plots in the inset show magnifications at medium and high frequencies. Simulations in (b) represent the complete equivalent circuit for a thermoelectric module [Fig. 5.3(b)] under high vacuum $\left(h=0 \mathrm{WK}^{-1} \mathrm{~m}^{-2}\right)$ and room $\left(h \approx 25 \mathrm{WK}^{-}\right.$ ${ }^{1} \mathrm{~m}^{-2}$ ) conditions. $R_{\Omega}=0$ is considered for simplicity and typical values for commercial thermoelectric modules were used $\left(S=190 \mu \mathrm{VK}^{-1}, \lambda_{T E}=1.5 \mathrm{WK}^{-1} \mathrm{~m}^{-1}, \alpha_{T E}=0.37 \mathrm{~mm}^{2} \mathrm{~s}^{-1}, L=1.6 \mathrm{~mm}, \lambda_{C}=35 \mathrm{WK}^{-1} \mathrm{~m}^{-1}, \alpha_{C}=10\right.$ $\left.\mathrm{mm}^{2} \mathrm{~s}^{-1}, L_{C}=0.6 \mathrm{~mm}, N=127, A=1.94 \mathrm{~mm}^{2}, T_{i}=294.7 \mathrm{~K}, \eta=0.3\right)$.

Fig. 5.4(b) shows the total impedance response [equivalent circuit in Fig. 5.3(b)] for the cases of high vacuum $\left(h=0 \mathrm{WK}^{-1} \mathrm{~m}^{-2}\right)$ and room conditions $\left(h=25 \mathrm{WK}^{-1} \mathrm{~m}^{-2}\right)$ [12]. As previously discussed, the convection effect does not make any influence until mid-low frequencies, thus, the impedance response do not differ until these frequencies are reached, as observed in Fig. 5.4(b). It can be also observed from Fig. 5.4(b) that the main difference in the impedance response produced by the convection is a reduction of the semicircle. This reduction can be quantified by the dc (steady state) limit $(\omega \rightarrow 0)$, which is given by $R_{\Omega}+\left(R_{T E}{ }^{-1}+R_{\text {conv }}{ }^{-1}\right)^{-1}$, being $R_{T E}=2 N S^{2} T_{i} L /\left(\lambda_{T E} A\right)$, and becomes reduced with respect to the high vacuum (adiabatic) case $\left(R_{\Omega}+R_{T E}\right)$ by the presence of $R_{\text {conv }}$. It should be noticed that if the module $Z_{m} T=R_{T E} / R_{\Omega}$ is calculated by IS $[6,13]$ at room conditions by considering $R_{T E}$ as the difference between the low and high frequency intercepts of the spectrum with the real impedance axis, or using the adiabatic 
equivalent circuit $\left(R_{\text {conv }}=0\right.$ ), a lower value of $R_{T E}$ will be obtained as shown in Fig. 5.4(b), consequently producing an underestimation of the $Z_{m} T$.

\subsection{Experimental validation}

In order to validate the new equivalent circuit [Fig. 5.3(b)], impedance measurements were performed to a $40 \mathrm{~mm} \times 40 \mathrm{~mm}$ commercial TE module from Interm (Ref. TECB1-1, power generation) formed by 127 couples with $1.4 \mathrm{~mm} \times 1.4 \mathrm{~mm} \times 1.64 \mathrm{~mm} \mathrm{legs} \mathrm{and} 0.57 \mathrm{~mm}$ of ceramic thickness. Two different measurements were performed to the module under suspended conditions in a vertical position. A first measurement was carried out at room conditions (ambient pressure) and a second one under high vacuum $\left(3.4 \times 10^{-5}\right.$ mbar) in order to completely remove convection losses. These impedance measurements were performed using a PGSTAT30 potentiostat equipped with a FRA2 impedance module (Metrohm Autolab B. V.) at 0 A dc current and $40 \mathrm{~mA}$ ac current amplitude, employing a frequency range from $10 \mathrm{kHz}$ to $10 \mathrm{mHz}$. All the measurements were performed inside a vacuum chamber and at the same ambient temperature of $21.2^{\circ} \mathrm{C}$

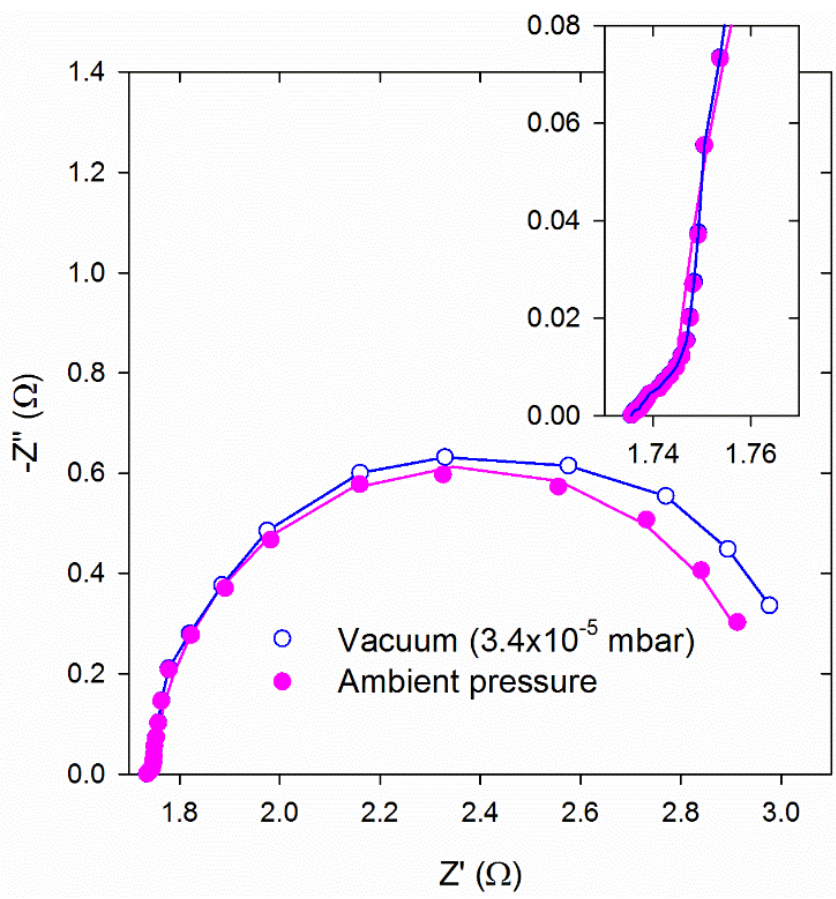

Fig. 5.5. Experimental impedance spectra of a suspended commercial thermoelectric module at room (ambient pressure) and high vacuum conditions (dots) and their corresponding fittings to the equivalent circuit of Fig. 5.3(b) (lines). The convection resistance was not considered for the vacuum case ( $h \approx 0 \mathrm{WK}^{-}$ 
${ }^{1} \mathrm{~m}^{-2}$ ). The quantity of $13.8 \mathrm{~m} \Omega$ was subtracted to the real impedance part of the vacuum measurement in order to match the ohmic resistances $\left(R_{\Omega}\right)$ and obtain a clearer comparison.

Fig. 5.5 shows the experimental measurements and their corresponding fitting to the equivalent circuit of Fig. 5.3(b). Zview software was used to perform the fittings. It can be observed that the results obtained are quite similar to the simulations performed using the theoretical model [Fig. 5.4(b)]. As expected from the above theoretical analysis, both measurements overlap at the highest frequencies (inset of Fig. 5.5) and the differences due to the effect of convection appear at the mid-low frequencies.

Since $h \approx 0 \mathrm{WK}^{-1} \mathrm{~m}^{-2}$ for the measurement under high vacuum, the fitting to this result was performed considering $R_{\text {conv }}=0$, which allows obtaining $R_{\Omega}, R_{T E}, \omega_{T E}, R_{C}\left[R_{C}=4 N S^{2} T_{i} L_{C}\left(\lambda_{C} A\right)\right]$, and $\omega_{C}$, whose values are shown in Table 5.1. The resistances $R_{T E}$ and $R_{C}$ depend on the properties of the materials, which do not change with the pressure of the environment. These two resistances were fixed when the fitting was performed to the high vacuum measurement, otherwise the fitting is not possible and very high errors will be obtained. All the fitting results are shown in Table 5.1. As it can be seen from Eq. (5.13), $h$ can be obtained from $R_{c o n v}$ if the average Seebeck coefficient of the module is known. In order to determine the value of $h$ in this way, the Seebeck coefficient of the module was measured from an open-circuit voltage vs. temperature difference curve, obtaining a value of $192.14 \mu \mathrm{VK}^{-1}$ (see Table 5.1). Using this value of $S$, a convection heat transfer coefficient $h=40.12 \mathrm{WK}^{-1} \mathrm{~m}^{-2}$ was obtained, which although somewhat higher than the typical values for natural convection at ambient pressure $\left(2-25 \mathrm{WK}^{-1} \mathrm{~m}^{-2}[12]\right)$ it is not very far and in the same order of magnitude.

Table 5.1. Fitting parameters obtained from the fittings to the experimental measurements in Fig. 5.5 of a commercial thermoelectric module under high vacuum and ambient pressure conditions (no vacuum). The errors provided from the fitting are given in brackets. The Seebeck coefficient was experimentally obtained and the convection heat transfer coefficient calculated from the no vacuum fitting result.

\begin{tabular}{|c|c|c|c|c|c|c|c|c|}
\hline & $R \Omega(\Omega)$ & $\boldsymbol{R}_{T E}(\mathbf{\Omega})$ & $\begin{array}{c}\omega T E \\
\left(\operatorname{rads}^{-1}\right)\end{array}$ & $R_{C}(\Omega)$ & $\begin{array}{c}\omega C \\
\left(\operatorname{rads}^{-1}\right)\end{array}$ & $\begin{array}{c}\boldsymbol{R}_{\text {conv }} \\
(\boldsymbol{\Omega})\end{array}$ & $\begin{array}{c}S \\
\left(\mu V K^{-1}\right)\end{array}$ & $\begin{array}{c}h \\
\left(\mathbf{W K}^{-1} \mathbf{m}^{-2}\right)\end{array}$ \\
\hline Vacuum & $\begin{array}{c}1.75 \\
(0.09 \%)\end{array}$ & $\begin{array}{c}1.33 \\
(0.37 \%)\end{array}$ & $\begin{array}{c}0.33 \\
(10.3 \%)\end{array}$ & $\begin{array}{c}0.036 \\
(25.9 \%)\end{array}$ & $\begin{array}{c}11.72 \\
(26.7 \%)\end{array}$ & --- & 192.14 & --- \\
\hline $\begin{array}{c}\text { No } \\
\text { vacuum }\end{array}$ & $\begin{array}{c}1.74 \\
(0.06 \%)\end{array}$ & --- & $\begin{array}{c}0.32 \\
(9.5 \%)\end{array}$ & --- & $\begin{array}{c}11.66 \\
(3.0 \%)\end{array}$ & $\begin{array}{c}21.75 \\
(6.1 \%)\end{array}$ & --- & 40.12 \\
\hline
\end{tabular}

A possible reason for the deviation observed could be attributed to convection effects that are also produced in other parts of the TE module, such as the surface at the side of the ceramics 
where the thermoelements are attached that is not covered by them. It should be noted that the mentioned approach to determine $h$ when the properties of the TE module are known can potentially offer the possibility to use TE modules as $h$ sensors.

On the other hand, if the value of $h$ of the measurement system is known $\left(40.12 \mathrm{WK}^{-1} \mathrm{~m}^{-2}\right.$ in our case), the Seebeck coefficient can be obtained from the convection resistance if measurements under high vacuum (which provides $R_{T E}$ and $R_{C}$ ) and at room conditions are performed. Once $S$ is known from $R_{c o n v}$ all the average properties of the TE module can be obtained $\left(\lambda_{T E}\right.$ from $R_{T E}, R_{\Omega}$, $Z_{m} T$, and $\lambda_{C}$ from $R_{C}$ ), without the need of knowing the thermal conductivity of the ceramic plates, which was a requirement in a previous approach $[6,8]$.

As it was mentioned above, if the measurement at room conditions had been used to determine $Z_{m} T$, a value of $R_{T E}=1.25$ would have been obtained, instead of the correct value from the vacuum measurement (1.33). This provides a $Z_{m} T=0.718$ which is $6 \%$ lower than the accurate vacuum result (0.764). Hence, in order to accurately determine $Z_{m} T$ from measurements under room conditions, the new model developed here [Fig. 5.3(b)] should be employed.

\subsection{Conclusions}

A new theoretical model (equivalent circuit) to interpret IS measurements of TE modules has been developed. The new model includes convection effects at the outer surface of the module ceramic plates, which introduces three new elements $\left(R_{\text {conv }}, Z_{W C T, C}\right.$ and $\left.C_{\text {conv }}\right)$ in the equivalent circuit. However, for standard commercial TE modules the equivalent circuit can be simplified when $h<500 \mathrm{WK}^{-1} \mathrm{~m}^{-2}$, only requiring to introduce the convection resistance $\left(R_{\text {conv }}\right)$. This new element, which depends on the convection heat transfer coefficient $h$, influences the mid-low frequency part of the spectrum, producing a reduction of the typically observed semicircle. The theoretical model was experimentally validated by performing IS measurements under high vacuum and at room conditions (ambient pressure) to a commercial TE module. The experimental measurements were found to be in agreement with the predicted results from the theoretical model and allowed the determination of the convection heat transfer coefficient, which was in the same order of magnitude than literature values.

The determination of the convection heat transfer coefficient is possible if the module average Seebeck coefficient is known and an impedance measurement in vacuum is performed, which 
opens up the possibility to develop TE modules as $h$ sensors. On the other hand, if $h$ is known, all the properties of the TE module $\left(Z_{m} T\right.$, ohmic (internal) resistance, thermal conductivity of the ceramics, average Seebeck coefficient and average thermal conductivity of the thermoelements) can be obtained from one impedance measurement in vacuum and another measurement at room conditions. A final analysis also showed that an underestimation of the module $Z_{m} T$ of $6 \%$ can be produced if the new equivalent circuit is not employed when the characterization of the TE module is performed at room conditions.

\section{Acknowledgments}

The authors acknowledge financial support from the Spanish Agencia Estatal de Investigación under the Ramón y Cajal program (RYC-2013-13970), from the Universitat Jaume I under the project UJI-A2016-08, and the technical support of Raquel Oliver Valls and José Ortega Herreros.

\section{References}

[1] A. De Marchi, V. Giaretto, S. Caron, A. Tona, A. De Marchi, V. Giaretto, S. Caron, A. Tona, A Novel zT Meter Based on the Porcupine Method and a Survey on the Size of the Snout Correction Needed for Various Thermoelectric Devices, J. Electron. Mater. 42 (2013) 2067-2072. https://doi.org/10.1007/s11664-013-2530-2.

[2] A. De Marchi, V. Giaretto, An accurate new method to measure the dimensionless figure of merit of thermoelectric devices based on the complex impedance porcupine diagram, Rev. Sci. Instrum. 82 (2011). https://doi.org/10.1063/1.3656074.

[3] G. Min, T. Singh, J. García-Cañadas, R. Ellor, Evaluation of Thermoelectric Generators by I-V Curves, J. Electron. Mater. 45 (2016) 1700-1704. https://doi.org/10.1007/s11664015-4180-z.

[4] Y. Hasegawa, R. Homma, M. Ohtsuka, Thermoelectric Module Performance Estimation Based on Impedance Spectroscopy, J. Electron. Mater. 45 (2016) 1886-1893. https://doi.org/10.1007/s11664-015-4271-x.

[5] M. Otsuka, H. Terakado, R. Homma, Y. Hasegawa, M.Z. Islam, G. Bastian, A. Stuck, Thermal diffusivity measurement using thermographic method and performance 
evaluation by impedance spectroscopy for thermoelectric module, in: Jpn. J. Appl. Phys., Japan Society of Applied Physics, 2016. https://doi.org/10.7567/JJAP.55.126601.

[6] J. García-Cañadas, G. Min, Chapter 6. High-throughput Thermoelectric Measurement Techniques, in: Thermoelectr. Mater. Devices, Royal Society of Chemistry, Cambridge, 2016: pp. 133-155. https://doi.org/10.1039/9781782624042-00133.

[7] M. Otsuka, Y. Hasegawa, T. Arisaka, R. Shinozaki, H. Morita, Dimensionless figure of merit and its efficiency estimation for transient response of thermoelectric module based on impedance spectroscopy, Appl. Phys. Express. 10 (2017) 115801. https://doi.org/10.7567/APEX.10.115801.

[8] J. García-cañadas, G. Min, Impedance spectroscopy models for the complete characterization of thermoelectric materials, J. Appl. Phys. 116 (2014). https://doi.org/10.1063/1.4901213.

[9] C.Y. Yoo, Y. Kim, J. Hwang, H. Yoon, B.J. Cho, G. Min, S.H. Park, Impedance spectroscopy for assessment of thermoelectric module properties under a practical operating temperature, $\quad$ Energy. $\quad 152 \quad$ (2018) 834-839. https://doi.org/10.1016/j.energy.2017.12.014.

[10] J. García-Cañadas, G. Min, Thermal dynamics of thermoelectric phenomena from frequency resolved methods, AIP Adv. $6 \quad$ (2016) 035008. https://doi.org/10.1063/1.4943958.

[11] F. Casalegno, A. De Marchi, V. Giaretto, Frequency domain analysis of spreadingconstriction thermal impedance, Rev. Sci. Instrum. $84 \quad$ (2013) 024901. https://doi.org/10.1063/1.4789765.

[12] F.P. Incropera, D.P. DeWitt, Fundamentals of Heat and Mass Transfer, John Wiley \& Sons, USA, 2002.

[13] A.D. Downey, T.P. Hogan, B. Cook, Characterization of thermoelectric elements and devices by impedance spectroscopy, Rev. Sci. Instrum. 78 (2007) 093904. https://doi.org/10.1063/1.2775432. 
6. Characterization of thermal contacts between heat exchangers and a thermoelectric module by impedance spectroscopy 


\title{
Characterization of thermal contacts between heat exchangers and a thermoelectric module by impedance spectroscopy
}

\author{
Braulio Beltran-Pitarch, Francisco Vidan, Jorge García-Cañadas*
}

Department of Industrial Systems Engineering and Design, Universitat Jaume I, Campus del Riu Sec, 12071 Castellón, Spain

*e-mail: garciaj@uji.es 


\begin{abstract}
Heat to electricity energy conversion efficiency of a thermoelectric (TE) device is not only influenced by the TE materials properties, but it also depends on the temperature difference between both sides of the TE legs. Keeping this temperature difference as close as possible to the temperature difference between the heat sink and the heat source is crucial to maximize the TE device performance. However, achieving this is quite difficult, mainly due to the thermal contact resistance at the interfaces between the TE module and the heat sink/source. In this study, it is analyzed the effect of this thermal contact resistance on the impedance spectroscopy (IS) response of a TE module that is thermally contacted by two aluminum blocks, which act as heat exchangers. A new theoretical model (equivalent circuit) that takes into account the thermal contact resistance is developed, which includes two new elements that depend on this parameter. The equivalent circuit is tested with experimental impedance measurements where the thermal contact is varied. It is demonstrated that using this equivalent circuit the thermal contact resistivity can be easily determined, which opens up the possibility of using IS as a tool to quantify and monitor this crucial property for the TE device performance.
\end{abstract}




\section{Graphical abstract}

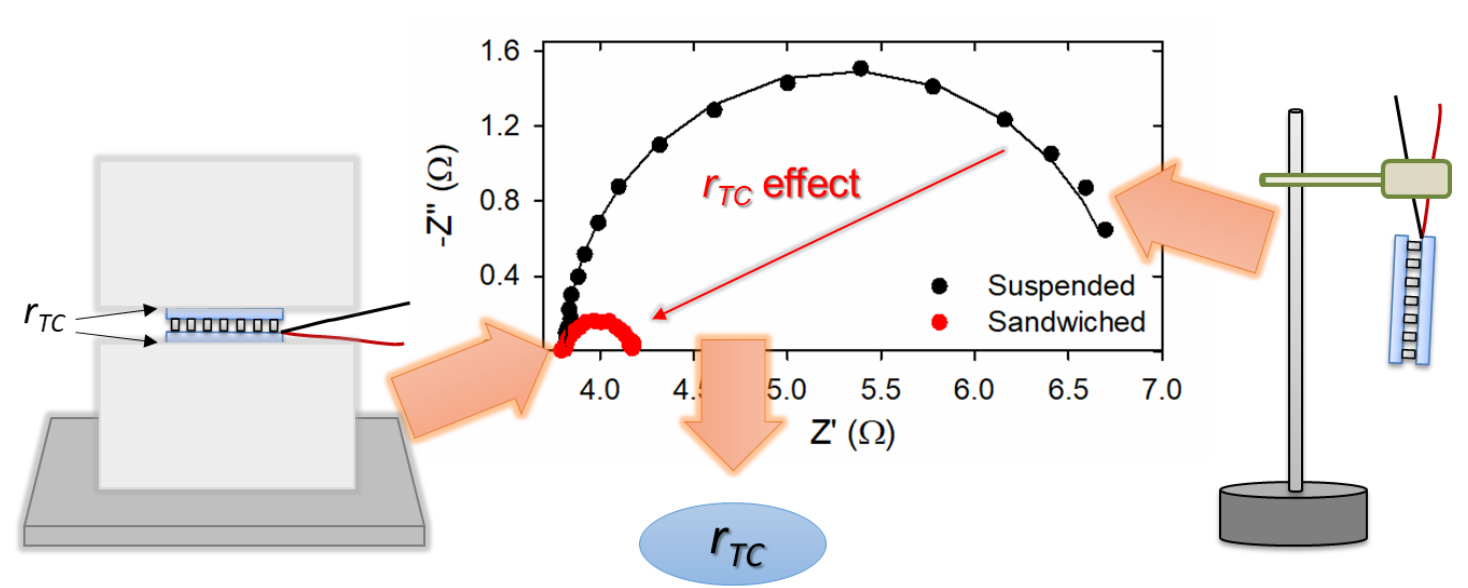

Fig. 6.1. Graphical abstract of the work: Characterization of thermal contacts between heat exchangers and a thermoelectric module by impedance spectroscopy. 


\subsection{Introduction}

A thermoelectric (TE) device that operates converting heat into electricity is typically contacted to a heat source and a heat sink under operating conditions. The heat to electricity energy conversion efficiency of the device depends on the TE properties of its TE materials, and also on the temperature difference at their edges. In the latter case, in order to maximize the efficiency it is important to keep the temperature at the edges of the TE materials as close as possible to the temperature of the heat sink and the heat source contacted at each of the sides of the device. Due to the thermal contact resistance existing at the interface between two solids [1], the temperature at the surface of the heat exchangers differs from that at the surface of the alumina ceramics (or similar electrically insulating material) which are usually the most external layers of a TE device. In order to minimize the thermal contact resistance different parameters can significantly influence, such as the contact pressure and the roughness of the surfaces that enter into contact [2,3]. In addition, it is typically adopted the use of different thermal interface materials (e.g. thermal grease, graphite sheets), which can significantly decrease the thermal contact resistance and thus increase the system efficiency [2-4].

The thermal contact resistance between two materials is typically determined by measuring the temperature drop at the interface of the materials when heat flows through the interface. This is performed using several thermocouples and materials with known thermal conductivity [5-8]. In many cases, it is also common the use of an infrared camera instead of thermocouples to determine the temperature profile across the junction $[9,10]$. Alternatively, thermal contact resistances can be also determined by fitting the temperature profile recorded in one solid when another solid in contact is heated by a laser beam $[11,12]$.

We present here a new method to determine the thermal contact resistivity between the external surfaces of a TE device and the heat exchangers. The method is based on the use of IS and it has recently received significant attention in the field of TEs due to its capability to characterize TE materials and modules [13-18]. Especially interesting are different studies that have shown the high sensitivity of the impedance method to any thermal phenomena taking place at the surroundings of the TE module, such as convection [15,19], radiation [15], or conduction through other material in contact with the module [20,21]. Due to this, it is expected that a thermal contact resistance will also produce a significant influence in the impedance response, and in fact 
this was observed in a recent study [22]. However, this recent article did not develop any equivalent circuit to account for the thermal contact resistance influence. On the other hand, a previous study [21] considered the presence of a thermal contact in the impedance response in the context of a thermal quadrupole analysis, but a detailed analysis of the thermal contact resistance and a procedure for its determination was missing.

In this work, a new impedance theoretical model (equivalent circuit), which includes the presence of thermal contact resistances between heat exchangers and the outer ceramic surfaces of a TE module has been developed and experimentally tested. The new equivalent circuit is obtained by solving the heat equation in the frequency domain. Using this equivalent circuit, the thermal contact resistivities between the heat exchangers and the TE module can be determined from an IS measurement of the module under suspended conditions and another measurement of the module contacted with the heat exchangers, given that the average Seebeck coefficient of the device thermoelements is known.

\subsection{Theoretical model}

The theoretical model adopted for the interpretation of the impedance response with the presence of thermal contact resistances is shown in Fig. 6.2. This one-dimensional model consists of $2 N$ TE legs of certain length $L$ and area $A$, being $N$ the number of TE couples. Each leg is sandwiched between two ceramic pieces of length $L_{C}$ and an area per TE leg $A / \eta$, being $\eta$ the filling factor of the TE module, which is the ratio between the area of the ceramic plate occupied by the TE legs $[(2 N+v) A$, being $v$ the number of legs removed to attach the leads of the device, typically 2] and the actual area of the ceramics. The thermal influence of the metallic strips (usually copper electrodes) that interconnect the TE legs is neglected due to their high thermal conductivity and small thickness. The model does not consider spreading-constriction effects $[15,23]$ of the heat flow due to the difference of area between TE legs and ceramics for simplicity, but it considers the difference between the areas (introduced by the filling factor $\eta$ ). All the TE properties are considered independent on temperature and Joule effect is neglected due to the high electrical conductivity of TE materials and the small current amplitude used during the IS measurements. The system is also considered adiabatic (no radiation and convection losses). At 
the outer ceramic surfaces a thermal contact resistance and a perfect heat sink condition are considered (see Fig. 6.2).

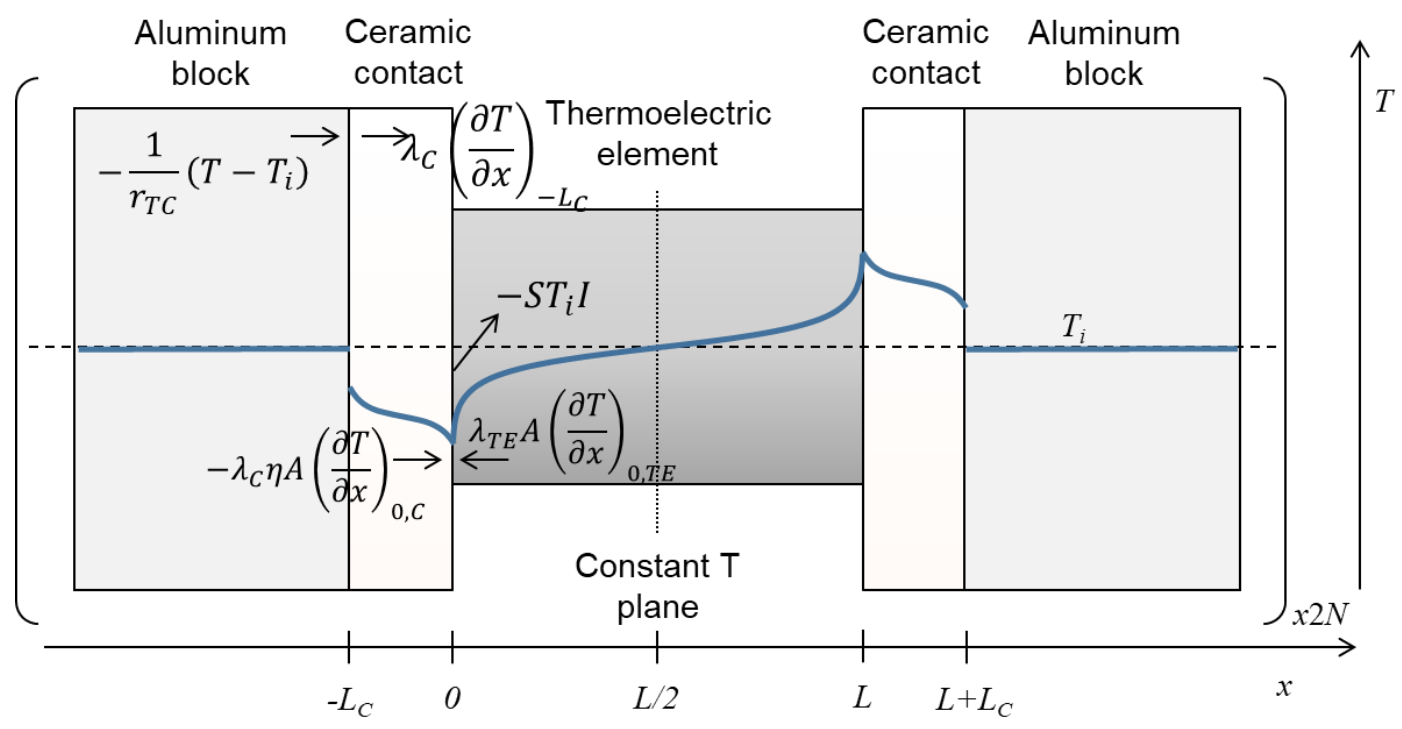

Fig. 6.2. Thermal model employed in the theoretical analysis. The arrows indicate the direction of the conducting heat fluxes appearing at the different junctions, considering a positive value of both the electrical current and the Seebeck coefficient. For the Peltier heat, the arrows point out of the junction when heat is absorbed. The solid line depicts qualitatively a possible thermal profile. The dotted line shows the plane where the temperature remains constant at any time due to the symmetry of the system. The dashed line indicates the initial temperature.

The impedance function $Z=\Delta V / I$ of a TE module as defined above and considering the symmetry of the system is given by,

$$
Z=\frac{\Delta V}{I_{0}}=R_{\Omega}+2 N \frac{S[T(L)-T(0)]}{I_{0}}=R_{\Omega}-2 N \frac{2 S\left[T(0)-T_{i}\right]}{I_{0}},
$$

where $\Delta V$ is the voltage difference across the TE device, $I_{0}$ is the electrical current flowing through the device at $x=0, R_{\Omega}$ is the total ohmic resistance, which includes the contribution of all the TE legs of the TE module, the metallic strips, the leads, and the electrical contact resistances, $S$ is the average absolute Seebeck coefficient of $n$ - and $p$-type thermoelements, and $T(0), T(L)$, and $T_{i}$ are the temperatures at $x=0, x=L$, and the initial temperature, respectively. It should be noted from Eq. (3.4) that due to the symmetry of the system with respect to the constant temperature plane $(x=L / 2)$, the temperature difference across the TE leg can be determined from the value of the temperature at $x=0$. 
To determine the variation with frequency of $T(0)$, the heat equation of the system must be solved in the frequency domain,

$$
\frac{\partial^{2} \theta}{\partial x^{2}}-\frac{j \omega}{\alpha_{i}} \theta=0
$$

where $\theta=L\left[T-T_{i}\right]$ is the Laplace transform of the temperature with respect to the initial temperature, $j=(-1)^{0.5}$ is the imaginary number, $\omega$ is the angular frequency (defined as $\omega=2 \pi f$, where $f$ is the frequency) and $\alpha_{i}$ is the average thermal diffusivity of the TE legs ( $i=\mathrm{TE}$ ) or the ceramic plates $(i=\mathrm{C})$.

The solution of Eq. (5.2) and its derivative are given by,

$$
\begin{aligned}
& \theta=C_{1, i} \sinh \left[\frac{x}{L_{i}}\left(\frac{j \omega}{\omega_{i}}\right)^{0.5}\right]+C_{2, i} \cosh \left[\frac{x}{L_{i}}\left(\frac{j \omega}{\omega_{i}}\right)^{0.5}\right], \\
& \frac{\partial \theta}{\partial x}=\frac{1}{L_{i}}\left(\frac{j \omega}{\omega_{i}}\right)^{0.5}\left\{C_{1, i} \cosh \left[\frac{x}{L_{i}}\left(\frac{j \omega}{\omega_{i}}\right)^{0.5}\right]+C_{2, i} \sinh \left[\frac{x}{L_{i}}\left(\frac{j \omega}{\omega_{i}}\right)^{0.5}\right]\right\},
\end{aligned}
$$

where $L_{i}$ is the half length of the TE legs $(i=\mathrm{TE})$ or the thickness of the ceramic plate $(i=\mathrm{C}), \omega_{i}$ is the characteristic angular frequency of each material (being $\omega_{i}=\alpha_{i} / L_{i}{ }^{2}$ ), and $C_{l, i}$ and $C_{2, i}$ are constants.

The following boundary conditions are applied (see Fig. 6.2),

$$
\begin{aligned}
& -\theta\left(-L_{C}\right)_{C}+r_{T C} \lambda_{C}\left(\frac{\partial \theta}{\partial x}\right)_{-L_{C}}=0, \text { at } x=-L_{C}, \\
& \theta(0)_{T E}=\theta(0)_{C}, \text { at } x=0, \\
& -S T_{i} i_{0}-\lambda_{C} \eta A\left(\frac{\partial \theta}{\partial x}\right)_{0, C}+\lambda_{T E} A\left(\frac{\partial \theta}{\partial x}\right)_{0, T E}=0, \text { at } x=0, \\
& \theta(L / 2)=0, \text { at } x=L / 2,
\end{aligned}
$$

where $i_{0}$ is the Laplace transform of the current $\left(i_{0}=L\left[I_{0}\right]\right)$ at $x=0$, and $\lambda_{C}$ and $\lambda_{T E}$ the thermal conductivity of the ceramic plates and the TE legs, respectively. In addition, $r_{T C}$ is the thermal contact resistivity between the TE module and the heat exchangers.

Using the boundary conditions given by Eqs. (5.5) to (5.8) in Eqs. (5.3) and (5.4), the Laplace transform of the temperature at $x=0$ takes the form, 


$$
\begin{aligned}
\theta(0)=\frac{-S T_{i} i_{0}}{A}\{ & \frac{\lambda_{T E}}{L_{T E}}\left(\frac{j \omega}{\omega_{T E}}\right)^{0.5} \operatorname{coth}\left(\frac{j \omega}{\omega_{T E}}\right)^{0.5} \\
& \left.+\frac{\lambda_{C}}{L_{C}}\left(\frac{j \omega}{\omega_{C}}\right)^{0.5} \frac{\cosh \left(\frac{j \omega}{\omega_{C}}\right)^{0.5}+\frac{r_{T C} \lambda_{C}}{L_{C}}\left(\frac{j \omega}{\omega_{C}}\right)^{0.5} \sinh \left(\frac{j \omega}{\omega_{C}}\right)^{0.5}}{\frac{r_{T C} \lambda_{C}}{L_{C}}\left(\frac{j \omega}{\omega_{C}}\right)^{0.5} \cosh \left(\frac{j \omega}{\omega_{C}}\right)^{0.5}+\sinh \left(\frac{j \omega}{\omega_{C}}\right)^{0.5}}\right\}^{-1}
\end{aligned}
$$

Using Eq. (3.4) and reorganizing the different terms, we reach the impedance expression,

$$
Z=R_{\Omega}+\left\{Z_{W C T}{ }^{-1}+\left[\left(Z_{W a}{ }^{-1}+R_{T C}{ }^{-1}\right)^{-1}+\left(Z_{W C T, C}{ }^{-1}+Z_{C_{T C}}{ }^{-1}\right)^{-1}\right]^{-1}\right\}^{-1},
$$

where the elements in Eq. (5.10) are,

$$
\begin{aligned}
& Z_{W C T}=\frac{2 N S^{2} T_{i} L}{\lambda_{T E} A}\left(\frac{j \omega}{\omega_{T E}}\right)^{-0.5} \tanh \left[\left(\frac{j \omega}{\omega_{T E}}\right)^{0.5}\right], \\
& Z_{W a}=\frac{4 N S^{2} T_{i} L_{C} \eta}{\lambda_{C} A}\left(\frac{j \omega}{\omega_{C}}\right)^{-0.5} \operatorname{coth}\left[\left(\frac{j \omega}{\omega_{C}}\right)^{0.5}\right], \\
& R_{T C}=\frac{4 N S^{2} T_{i} r_{T C} \eta}{A}, \\
& Z_{W C T, C}=\frac{4 N S^{2} T_{i} L_{C} \eta}{\lambda_{C} A}\left(\frac{j \omega}{\omega_{C}}\right)^{-0.5} \tanh \left[\left(\frac{j \omega}{\omega_{C}}\right)^{0.5}\right], \\
& Z_{C_{T C}}=\frac{4 N S^{2} T_{i} L_{C}{ }^{2} \eta}{\lambda_{C}{ }^{2} A r_{T C}}\left(\frac{j \omega}{\omega_{C}}\right)^{-1}=\frac{4 N S^{2} T_{i} \eta}{\lambda_{C} d_{C} C_{p, C} A r_{T C}} \frac{1}{j \omega},
\end{aligned}
$$

being $d_{C}$ and $C_{p, C}$ the mass density and specific heat of the ceramic material, respectively. Eq. (5.10) can be represented by the equivalent circuit shown in Fig. 6.3.

The new equivalent circuit of Fig. 6.3 has similarities to that obtained in our previous work where the TE module was simply suspended (without the presence of heat exchangers) and the effect of convection was considered at the outer ceramic surfaces [19]. Unlike the previous work, due to the presence of the heat exchangers here a thermal contact resistance boundary condition and no temperature variation in the heat exchangers is considered (see Fig. 6.2). This produces the existence of a thermal contact resistivity instead of the convection heat transfer coefficient in the resistance and capacitance elements $\left(R_{T C}\right.$ and $C_{T C}$, which in the case of convection were $R_{c o n v}$ and $C_{c o n v}$ ). In addition, the filling factor $\eta$, which in the previous article was added to account for 
convection effects produced at the external area of the ceramic plates that was larger than the total legs area, and only affected the $R_{\text {conv }}$ and $C_{\text {conv }}$ impedance elements, now it also affects the Warburg elements related to the ceramic plates $\left(Z_{W a}\right.$ and $\left.Z_{W C T, C}\right)$. This is due to the consideration of the difference in areas between all the legs and the ceramic plates [Fig. 6.2 and Eq. (5.7)], as also occurred when this was considered in another of our previous studies [15]. The inclusion of $\eta$ in the elements related to the ceramic plates must be used as a general rule, since in this way the thermal effects in the total area of the ceramic plates is considered, not only in the part covered by the TE legs.

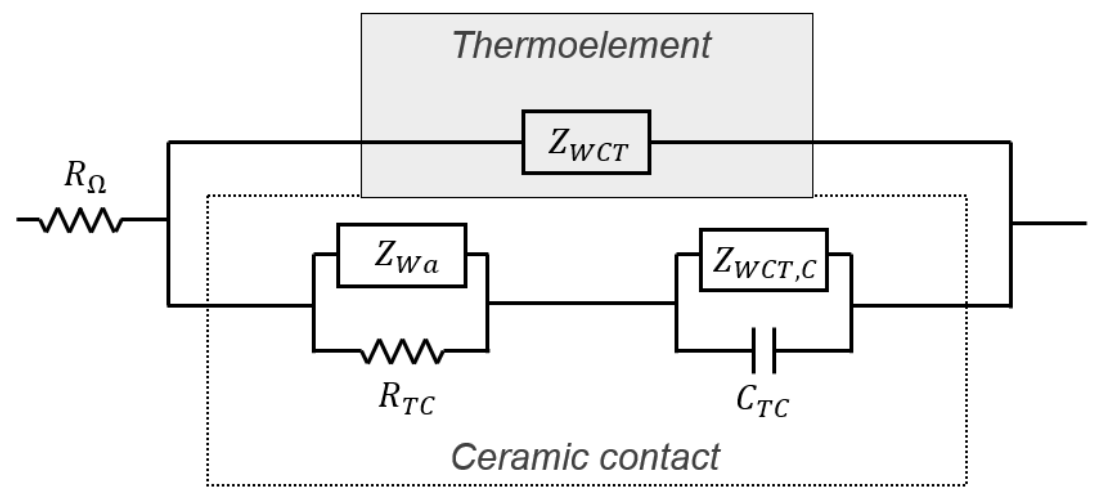

Fig. 6.3. Equivalent circuit corresponding to a thermoelectric device contacted by two heat sinks, existing a thermal contact resistance at the contact. The equivalent circuit elements framed by the dotted line are related to the external ceramic plates. The elements framed by the solid line relate to the thermoelements.

It can be observed that for the case of an ideal thermal contact $\left(r_{T C} \rightarrow 0\right)$, the impedance from the equivalent circuit element $R_{T C} \rightarrow 0$ and $C_{T C} \rightarrow \infty$, thus, the equivalent circuit in Fig. 6.3 reduces to the parallel combination of $Z_{W C T, C}$ with $Z_{W C T}$, connected in series with $R_{\Omega}$, whose response can be observed in the simulations on Fig. 6.4 (more clearly in the magnification of Fig. 6.4c). In this case, the constant temperature Warburg element from the ceramics $Z_{W C T, C}$ represents the diffusion of heat within the ceramic from the $\mathrm{Cu} / \mathrm{TE}$ junctions, which is completely removed by the effect of the heat sinks (heat exchangers) when they are reached, producing no temperature change at the outer ceramic surfaces (constant-temperature boundary). In the opposite case, if $r_{T C} \rightarrow \infty$, then $R_{T C} \rightarrow \infty$ and $C_{T C} \rightarrow 0$, and the equivalent circuit of Fig. 6.3 will reduce to the parallel combination of $Z_{W a}$ with $Z_{W C T}$, connected in series with $R_{\Omega}$, which is the response obtained for a suspended module under adiabatic conditions [13], as shown in the simulations of Fig. 6.4. In this case, the huge thermal contact resistivity blocks the heat transfer towards the heat exchangers and all the 
heat is accumulated in the ceramic layers. Apart from these two extreme cases, it can be observed from the simulations of Fig. 6.4, where the thermal contact resistivity value has been systematically reduced, that the presence of the thermal contact resistivity introduces significant differences in the semicircle part of the impedance response, which considerably reduces when $r_{T C}$ decreases. However, at high frequencies (bottom left part), the 45 degrees straight line feature is the same in all cases, which relates to the diffusion of heat from the TE legs ends towards the ceramic layers, until the end of the ceramic is reached. Once the effect of this boundary is sensed, around the turnover angular frequency $\omega=2 \pi \omega_{C}$ (see Fig. 6.4c), the effect of the thermal contact resistivity starts to be observed when the frequencies are decreased (moving to the right side).
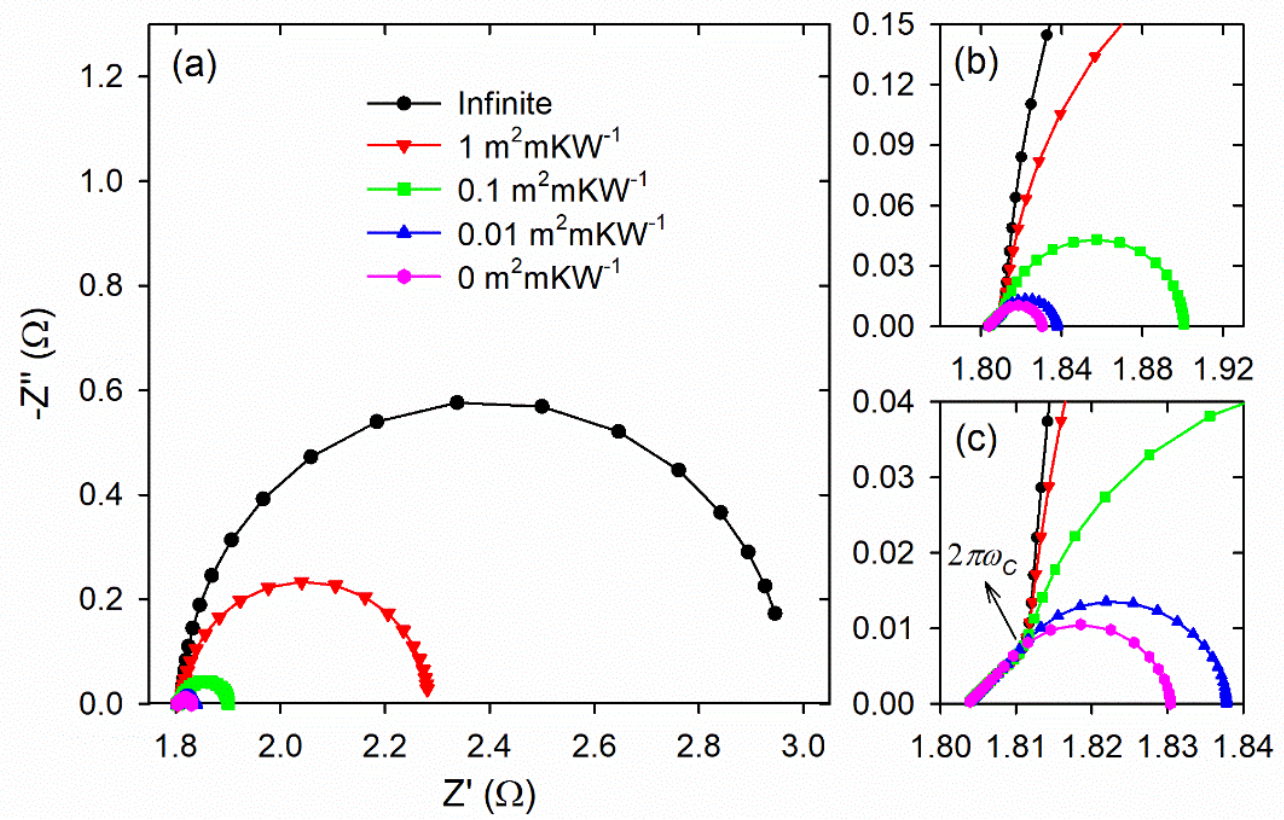

Fig. 6.4. (a) Impedance spectroscopy simulations from $10 \mathrm{mHz}$ to $10 \mathrm{kHz}$ for four different thermal contact resistivity values for the contacts between a thermoelectric module and two heat sinks. The plots in (b) and (c) are magnifications of the bottom left part (same axis units). Typical values for commercial $\mathrm{Bi}_{2} \mathrm{Te}_{3}$ thermoelectric modules were used $\left(N=127, S=180 \mu \mathrm{VK}^{-1}, \rho_{T E}=1 \mathrm{~m} \Omega \mathrm{cm}, \lambda_{T E}=1.5 \mathrm{Wm}^{-1} \mathrm{~K}^{-1}, \alpha_{T E}=0.37 \mathrm{~mm}^{2} \mathrm{~s}^{-}\right.$ $\left.{ }^{1}, L=1.2 \mathrm{~mm}, \lambda_{C}=20 \mathrm{Wm}^{-1} \mathrm{~K}^{-1}, \alpha_{C}=10 \mathrm{~mm}^{2} \mathrm{~s}^{-1}, L_{C}=0.7 \mathrm{~mm}, A=1.69 \mathrm{~mm}^{2}, T=300 \mathrm{~K}, \eta=0.268\right)$. Frequency decreases from left to right.

In the new equivalent circuit (Fig. 6.3), two new elements appear for the first time, $R_{T C}$ and $C_{T C} . R_{T C}=4 N S^{2} T_{i} r_{\mathrm{TC}} \eta / A$ is the thermal contact electrical resistance, in which the only thermal parameter that influences its value is $r_{T C}$. Its physical meaning is related to the electrical losses in the system due to the blockage of the heat flow by the thermal contacts, which causes a higher 
temperature modification of the temperature difference at the edges of the thermoelements, since heat removal by the heat exchangers is less efficient. High $r_{T C}$ values will considerably block the heat flow and high $R_{T C}$ values will result, as shown in Fig. 6.4a. In contrast, low $r_{T C}$ values will allow the heat to flow and very small $R_{T C}$ values will result. It should be noted that $R_{T C}$ has a huge influence on the dc resistance $R_{d c}$, which accounts for the total losses of the system. Note that $R_{d c}$ is the value adopted by the impedance response [Eq. (5.10)] when $\omega \rightarrow 0$ (steady state), and corresponds to $R_{d c}=R_{\Omega}+\left[R_{T E}{ }^{-1}+\left(R_{T C}+R_{C}\right)^{-1}\right]^{-1}$, being $R_{T E}=2 N S^{2} T_{i} L /\left(\lambda_{T E} A\right)$ the TE resistance, coming from the $Z_{W C T}$ element, and $R_{C}=4 N S^{2} T_{i} L_{C} \eta /\left(\lambda_{C} A\right)$ the TE resistance induced by the ceramic layers, coming from the $Z_{W C T, C}$ element $[13,24]$. $R_{d c}$ can be easily identified from the impedance plots, since it is the low frequency intercept with the real axis. It is remarkable that $R_{d c}$ is also influenced here by $R_{C}$, which was not the case in the impedance response of modules under suspended conditions $[13,15,19]$.

The other new element is a thermal contact capacitance $C_{T C}=\lambda_{C} d_{C} C_{p, C} A r_{T C} /\left(4 N S^{2} T_{i} \eta\right)$ since adopts the form of a capacitor $\left[Z_{C T C}=\left(j \omega C_{T C}\right)^{-1}\right]$. It is influenced by two thermal parameters, $r_{T C}$ and the thermal effusivity $e_{C}=\left(\lambda_{C} d_{C} C_{p, C}\right)^{0.5}$. The latter is a parameter that define the ability of materials to exchange heat with the surroundings [25]. Both $r_{T C}$ and $e_{C}$ determine the temperature of the junction at the side of the ceramic layer, which is eventually governed by the heat release/accumulation at the interface. When $r_{T C}$ increases, $C_{T C}$ also increases and the impedance response shows a growth in their absolute imaginary part values at $\omega<<2 \pi \omega_{C}$ (see Fig. 6.4), which is related to a higher temperature drop at the interface, produced by a more prominent heat release/accumulation. Although the effect of the variation of $e_{C}$ is not shown in Fig. 6.4, it will also provide a higher $C_{T C}$ value if increased, producing the same result as the $r_{T C}$ increase.

Using $R_{T E}, R_{C}$, and $R_{T C}$, the equivalent circuit elements of Eqs. (6.11), (6.12), (5.14) and (5.15), can be rewritten as,

$$
\begin{aligned}
& Z_{W C T}=R_{T E}\left(\frac{j \omega}{\omega_{T E}}\right)^{-0.5} \tanh \left[\left(\frac{j \omega}{\omega_{T E}}\right)^{0.5}\right], \\
& Z_{W a}=R_{C}\left(\frac{j \omega}{\omega_{C}}\right)^{-0.5} \operatorname{coth}\left[\left(\frac{j \omega}{\omega_{C}}\right)^{0.5}\right], \\
& Z_{W C T, C}=R_{C}\left(\frac{j \omega}{\omega_{C}}\right)^{-0.5} \tanh \left[\left(\frac{j \omega}{\omega_{C}}\right)^{0.5}\right],
\end{aligned}
$$




$$
Z_{C_{T C}}=\frac{R_{C}{ }^{2}}{R_{T C}}\left(\frac{j \omega}{\omega_{C}}\right)^{-1} .
$$

In this way, $C_{T C}$ can be obtained once $R_{C}$ and $R_{T C}$ are known, which then simplifies the fitting, since $C_{T C}$ is no longer a variable. Hence, these equations will be used to perform fittings to experimental results. This simplification cannot be easily implemented in the standard impedance fittings programs (e.g. Zview), and hence, Matlab will be employed for this purpose.

\subsection{Experimental results}

In order to experimentally test the model developed in the previous section, we performed impedance measurements using a $10 \mathrm{~mm} \times 10 \mathrm{~mm} \mathrm{Bi}_{2} \mathrm{Te}_{3}$ commercial TE module from Custom Thermoelectrics (Ref. 04801-9A30-18RB), which is formed by 48 couples of $0.6 \mathrm{~mm} \times 0.6 \mathrm{~mm}$ $\times 1.05 \mathrm{~mm}$ legs and $0.53 \mathrm{~mm}$ ceramic thickness. A first IS measurement was performed to this module suspended in vacuum conditions at a pressure of $3.0 \times 10^{-4} \mathrm{mbar}$ to eliminate convection losses [see Fig. 6.5(a)]. Then, the TE module was measured in air (without vacuum) sandwiched between two aluminum blocks (Cu alloy 2030), which acted as heat exchangers [see Fig. 6.5(b)].

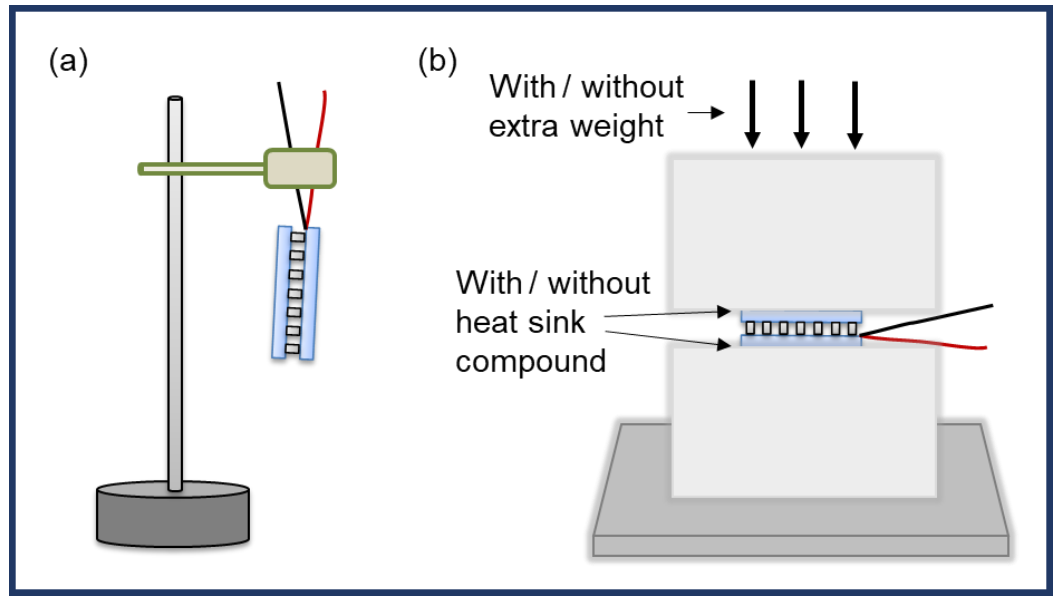

Fig. 6.5. Schematic of the experimental setups for the measurements performed to (a) a module suspended, and (b) the same module in contact with two aluminum blocks acting as heat exchangers and contacted with and without heat sink compound, and with and without additional weight on top.

The aluminum blocks are $50 \mathrm{~mm} \times 90 \mathrm{~mm} \times 100 \mathrm{~mm}$ size and have a mass of $1.2 \mathrm{~kg}$, which implies a pressure in the system of $118 \mathrm{kPa}$. The TE module sandwiched by the Al blocks was measured without (only mechanical contact) and with heat sink compound (thermal grease) from 
RS (Ref. 217-3835) added at the contacts. Additionally, two measurements with additional masses of $3.1 \mathrm{~kg}$, and $6.8 \mathrm{~kg}$ on top of the top aluminum block were performed to increase the pressure to $422 \mathrm{kPa}$, and $785 \mathrm{kPa}$, respectively. In order to have a good initial thermal contact, the aluminum blocks were slightly polished with 600 grit size silicon carbide sandpaper before being assembled. All the impedance measurements were performed using a PGSTAT30 potentiostat (Metrohm Autolab B. V.) equipped with a FRA2 impedance module at $0 \mathrm{~A}$ dc current and $30 \mathrm{~mA}$ ac current amplitude, measuring 40 logarithmically distributed frequency steps between $20 \mathrm{mHz}$ and $2 \mathrm{kHz}$. Fittings to the experimental results were performed using the equivalent circuit of Fig. 6.3 [Eqs. (6.16)-(6.19)] with Matlab software (the code is provided in Annex 9.1). All the measurements were performed inside a metallic vacuum chamber that acted as Faraday cage and at room temperature.

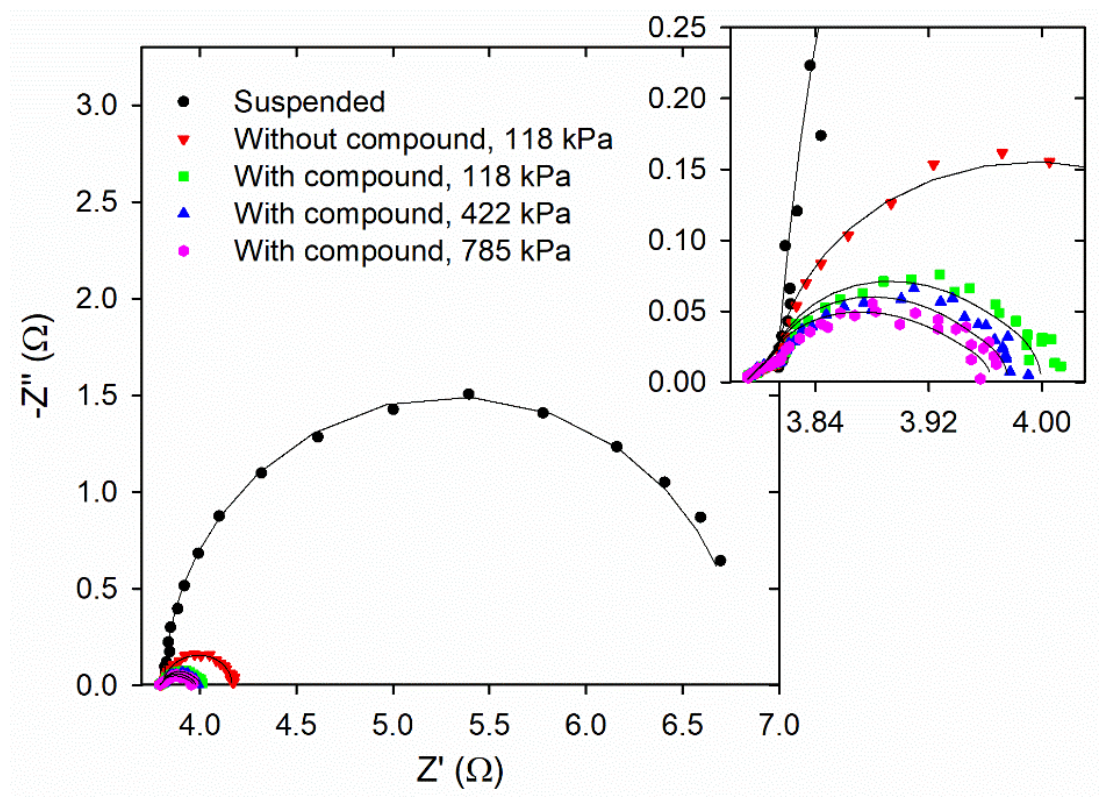

Fig. 6.6. Experimental impedance spectra (dots) of a commercial $\mathrm{Bi}_{2} \mathrm{Te}_{3}$ thermoelectric module suspended in vacuum and in contact in air with two aluminum blocks (with and without heat sink compound, and with additional masses on top of the top aluminum block). The lines represent the fittings performed with the equivalent circuit of Fig. 6.3. The inset shows the magnification at medium and high frequencies. Frequency decreases from left to right.

Fig. 6.6 shows the experimental IS measurements (dots) and their associated fittings (lines). It can be clearly observed that the same trend identified in the simulations from Fig. 6.4 occurs now in Fig. 6.6 as the thermal contact resistivity is modified. In addition, all the fittings performed are in good agreement with the experimental results. It should be noted that the fitting to the 
suspended module experiment was carried out discarding the effect of the thermal contact resistivity, i.e. with an equivalent circuit formed by the ohmic resistance $\left(R_{\Omega}\right)$ in series with the parallel combination of $Z_{W C T}$ and $Z_{W a}$ [13]. From this fitting, $R_{T E}$ and $R_{C}$ were obtained (see Table 6.1) and used in the fittings of the other experiments that include a thermal contact resistivity. This was performed since otherwise it is not possible to fit the complete equivalent circuit (Fig. 6.3) due to the large number of variables. Note that both $R_{T E}$ and $R_{C}$ only depend on materials properties, which do not change along the different setups. On the other hand, fittings adding the spreading-constriction impedance element, although initially discarded in the model, were tried, but they were also not possible due to the large number of variables.

Table 6.1. Fitting parameters obtained from the fittings to the experimental measurements of Fig. 6.6 of a commercial $\mathrm{Bi}_{2} \mathrm{Te}_{3}$ thermoelectric module suspended under vacuum and in contact with two aluminum blocks at room conditions $(\mathrm{T}=298.0 \mathrm{~K})$, with and without using heat sink compound, and with additional masses on top. The errors provided from the fittings are given between brackets. The thermal contact resistivities were calculated using Eq. (5.13) and the Seebeck coefficient value of $191.53 \mu \mathrm{VK}^{-1}$ was experimentally obtained.

\begin{tabular}{|c|c|c|c|c|c|c|c|c|}
\hline & $R_{\Omega}(\Omega)$ & $R_{T E}(\Omega)$ & $\begin{array}{c}\omega_{T E} \\
\left(\mathbf{r a d s}^{-1}\right) \\
\end{array}$ & $R_{C}(\Omega)$ & $\begin{array}{c}\omega_{C} \\
\left(\operatorname{rads}^{-1}\right) \\
\end{array}$ & $\begin{array}{l}C_{T C} \\
(\mathbf{F}) \\
\end{array}$ & $\boldsymbol{R}_{T C}(\mathbf{\Omega})$ & $\begin{array}{c}r_{T C} \\
\left(\mathbf{m}^{2} \mathbf{K W}^{-1}\right) \\
\end{array}$ \\
\hline Suspended & $\begin{array}{c}3.79 \\
(0.07 \%)\end{array}$ & $\begin{array}{c}3.02 \\
(0.32 \%)\end{array}$ & $\begin{array}{c}1.39 \\
(31.35 \%)\end{array}$ & $\begin{array}{c}0.083 \\
(20.55 \%)\end{array}$ & $\begin{array}{c}24.82 \\
(23.45 \%)\end{array}$ & --- & --- & --- \\
\hline $\begin{array}{c}\text { w/o } \\
\text { compound } \\
(118 \mathrm{kPa})\end{array}$ & $\begin{array}{c}3.77 \\
(0.03 \%)\end{array}$ & --- & $\begin{array}{c}0.48 \\
(10.39 \%)\end{array}$ & --- & $\begin{array}{c}27.14 \\
(3.78 \%)\end{array}$ & 1.83 & $\begin{array}{c}0.35 \\
(0.85 \%)\end{array}$ & $1.72 \times 10^{-4}$ \\
\hline $\begin{array}{c}\text { w/ } \\
\text { compound } \\
(118 \text { kPa })\end{array}$ & $\begin{array}{c}3.79 \\
(0.03 \%)\end{array}$ & --- & $\begin{array}{c}0.13 \\
(14.10 \%)\end{array}$ & --- & $\begin{array}{c}28.46 \\
(8.73 \%)\end{array}$ & 0.71 & $\begin{array}{c}0.14 \\
(2.02 \%)\end{array}$ & $7.01 \times 10^{-5}$ \\
\hline $\begin{array}{c}\text { w/ } \\
\text { compound } \\
(422 \mathrm{kPa})\end{array}$ & $\begin{array}{c}3.78 \\
(0.03 \%)\end{array}$ & --- & $\begin{array}{c}0.10 \\
(13.77 \%)\end{array}$ & --- & $\begin{array}{c}30.71 \\
(10.12 \%)\end{array}$ & 0.54 & $\begin{array}{c}0.11 \\
(2.28 \%)\end{array}$ & $5.68 \times 10^{-5}$ \\
\hline $\begin{array}{c}\text { w/ } \\
\text { compound } \\
(785 \text { kPa })\end{array}$ & $\begin{array}{c}3.78 \\
(0.04 \%)\end{array}$ & --- & $\begin{array}{c}0.054 \\
(15.73 \%)\end{array}$ & --- & $\begin{array}{c}47.61 \\
(20.99 \%)\end{array}$ & 0.31 & $\begin{array}{c}0.10 \\
(3.72 \%)\end{array}$ & $5.04 \times 10^{-5}$ \\
\hline
\end{tabular}

The fitting results to the experiments in Fig. 6.6 can be seen in Table 6.1. From the fitted value of $R_{T C}$ the thermal contact resistivity $r_{T C}$ can be determined if the average absolute Seebeck coefficient of the thermoelements is known [see Eq. (5.13)]. Hence, in order to determine $r_{T C}$, the Seebeck coefficient of the TE module was measured from an open-circuit voltage vs. temperature difference curve, obtaining a value of $191.53 \mu \mathrm{VK}^{-1}$. The obtained thermal contact resistivity values are shown in Table 6.1. The identified values are in good agreement with literature results $[4,8,26]$. It can be observed that a significant decrease in the thermal contact resistivity values 
takes place at $118 \mathrm{kPa}$ pressure when the heat sink compound is employed, which is clearly sensed by the impedance method. Moreover, when contact pressure was increased to 422 and $785 \mathrm{kPa}$, significant changes were also found in the impedance spectra (see Fig. 6.6) which translated into a decrease of the determined thermal contact resistivity values (see Table 6.1), and proves the validity of the method to track variations in the thermal contact resistance induced by different pressure levels.

\subsection{Conclusions}

In order to study the effect of a thermal contact resistance in the impedance signal of a TE module, a new theoretical model (equivalent circuit) has been developed considering a TE device sandwiched between two aluminum blocks, which act as heat exchangers. Two new elements in the equivalent circuit influenced by the thermal contact resistance appear in the analysis: a thermal contact electrical resistance and a thermal contact capacitance. The theoretical model is tested by experimental measurements performed to a commercial $\mathrm{Bi}_{2} \mathrm{Te}_{3}$ module contacted by aluminum blocks. It was found that the impedance response significantly differs from that of the module suspended (not in contact with the heat exchangers) under adiabatic conditions. The experimental results are in good agreement with the behavior predicted by the new theoretical model. Moreover, it is possible to quantify the value of the thermal contact resistivity. This can be performed from a measurement of the module in vacuum and the knowledge of the average Seebeck coefficient of its thermoelements before being measured in contact with the heat exchangers. Thermal contact resistivity values determined at different conditions (with and without thermal interface material and at different pressure levels) are in agreement with literature values. This opens up the possibility of using IS as a tool to quantify and monitor the thermal contact resistivity, even under reasonably small variations of the contact pressure, which is a key parameter in the performance of TE devices.

\section{Acknowledgments}

The authors acknowledge financial support from the Spanish Agencia Estatal de Investigación under the Ramón y Cajal program (RYC-2013-13970), from the Generalitat Valencian and the European Social Fund under the ACIF program (ACIF/2018/233) from the Universitat Jaume I 
under the project UJI-A2016-08, and the technical support of Raquel Oliver Valls and José Ortega Herreros. European Thermodynamics is also acknowledged for providing the thermoelectric devices.

\section{References}

[1] F.P. Incropera, D.P. DeWitt, Fundamentals of Heat and Mass Transfer, John Wiley \& Sons, USA, 2002.

[2] K. Karthick, S. Suresh, H. Singh, G.C. Joy, R. Dhanuskodi, Theoretical and experimental evaluation of thermal interface materials and other influencing parameters for thermoelectric generator system, Renew. Energy. 134 (2019) 25-43. https://doi.org/10.1016/j.renene.2018.10.109.

[3] S. Wang, T. Xie, H. Xie, Experimental study of the effects of the thermal contact resistance on the performance of thermoelectric generator, Appl. Therm. Eng. 130 (2018) 847-853. https://doi.org/10.1016/J.APPLTHERMALENG.2017.11.036.

[4] K. Karthick, G.C. Joy, S. Suresh, R. Dhanuskodi, Impact of Thermal Interface Materials for Thermoelectric Generator Systems, J. Electron. Mater. 47 (2018) 5763-5772. https://doi.org/10.1007/s11664-018-6496-y.

[5] M. Rosochowska, R. Balendra, K. Chodnikiewicz, Measurements of thermal contact conductance, J. Mater. Process. Technol. 135 (2003) 204-210. https://doi.org/10.1016/S0924-0136(02)00897-X.

[6] K. Azuma, T. Hatakeyama, S. Nakagawa, Measurement of surface roughness dependence of thermal contact resistance under low pressure condition, in: 2015 Int. Conf. Electron. Packag. IMAPS All Asia Conf., IEEE, 2015: pp. 381-384. https://doi.org/10.1109/ICEPIAAC.2015.7111040.

[7] P. Misra, J. Nagaraju, Test facility for simultaneous measurement of electrical and thermal contact resistance, Rev. Sci. Instrum. 75 (2004) 2625-2630. https://doi.org/10.1063/1.1775316.

[8] Y. Xian, P. Zhang, S. Zhai, P. Yuan, D. Yang, Experimental characterization methods for 
thermal contact resistance: A review, Appl. Therm. Eng. 130 (2018) 1530-1548. https://doi.org/10.1016/j.applthermaleng.2017.10.163.

[9] C. Fieberg, R. Kneer, Determination of thermal contact resistance from transient temperature measurements, Int. J. Heat Mass Transf. 51 (2008) 1017-1023. https://doi.org/10.1016/J.IJHEATMASSTRANSFER.2007.05.004.

[10] R.J. Warzoha, B.F. Donovan, High resolution steady-state measurements of thermal contact resistance across thermal interface material junctions, Rev. Sci. Instrum. 88 (2017) 094901. https://doi.org/10.1063/1.5001835.

[11] Y. Ohsone, G. Wu, J. Dryden, F. Zok, A. Majumdar, Optical Measurement of Thermal Contact Conductance Between Wafer-Like Thin Solid Samples, J. Heat Transfer. 121 (1999) 954. https://doi.org/10.1115/1.2826086.

[12] B. Dongmei, C. Huanxin, L. Shanjian, S. Limei, Measurement of thermal diffusivity/thermal contact resistance using laser photothermal method at cryogenic temperatures, Appl. Therm. Eng. $111 \quad$ (2017) 768-775. https://doi.org/10.1016/J.APPLTHERMALENG.2016.07.188.

[13] J. García-cañadas, G. Min, Impedance spectroscopy models for the complete characterization of thermoelectric materials, J. Appl. Phys. 116 (2014). https://doi.org/10.1063/1.4901213.

[14] B. Beltrán-Pitarch, J. Prado-Gonjal, A. V. Powell, P. Ziolkowski, J. García-Cañadas, Thermal conductivity, electrical resistivity, and dimensionless figure of merit (ZT) determination of thermoelectric materials by impedance spectroscopy up to $250{ }^{\circ} \mathrm{C}, \mathrm{J}$. Appl. Phys. 124 (2018) 025105. https://doi.org/10.1063/1.5036937.

[15] R. Mesalam, H.R. Williams, R.M. Ambrosi, J. García-Cañadas, K. Stephenson, Towards a comprehensive model for characterising and assessing thermoelectric modules by impedance spectroscopy, Appl. Energy. https://doi.org/10.1016/j.apenergy.2018.05.041.

[16] C.Y. Yoo, Y. Kim, J. Hwang, H. Yoon, B.J. Cho, G. Min, S.H. Park, Impedance spectroscopy for assessment of thermoelectric module properties under a practical 
Chapter 6

$\begin{array}{lllll}\text { operating } & \text { temperature, } & \text { Energy. } & 152 \quad \text { (2018) }\end{array}$

https://doi.org/10.1016/j.energy.2017.12.014.

[17] Y. Apertet, H. Ouerdane, Small-signal model for frequency analysis of thermoelectric systems, Energy Convers. Manag. $149 \quad$ (2017) 564-569. https://doi.org/10.1016/J.ENCONMAN.2017.07.061.

[18] Y. Hasegawa, M. Otsuka, Temperature dependence of dimensionless figure of merit of a thermoelectric module estimated by impedance spectroscopy, AIP Adv. 8 (2018) 075222. https://doi.org/10.1063/1.5040181.

[19] B. Beltrán-Pitarch, J. García-Cañadas, Influence of convection at outer ceramic surfaces on the characterization of thermoelectric modules by impedance spectroscopy, J. Appl. Phys. 123 (2018). https://doi.org/10.1063/1.5019881.

[20] B. Beltrán-Pitarch, L. Márquez-García, G. Min, J. García-Cañadas, Measurement of thermal conductivity and thermal diffusivity using a thermoelectric module, Meas. Sci. Technol. 28 (2017) 045902.

[21] A. De Marchi, V. Giaretto, The Peltier driven frequency domain approach in thermal analysis, Rev. Sci. Instrum. 85 (2014). https://doi.org/10.1063/1.4897189.

[22] S. Boldrini, A. Ferrario, A. Miozzo, Investigation of Pulsed Thermoelectric Performance by Impedance Spectroscopy, J. Electron. Mater. 48 (2019) 1805-1811. https://doi.org/10.1007/s11664-018-06922-9.

[23] F. Casalegno, A. De Marchi, V. Giaretto, Frequency domain analysis of spreadingconstriction thermal impedance, Rev. Sci. Instrum. $84 \quad$ (2013) 024901. https://doi.org/10.1063/1.4789765.

[24] J. García-Cañadas, G. Min, Low frequency impedance spectroscopy analysis of thermoelectric modules, J. Electron. Mater. 43 (2014) 2411-2414. https://doi.org/10.1007/s11664-014-3095-4.

[25] D.Y. Demezhko, V. V. Dergachev, E.N. Rybakov, A contact method of determining the thermal effusivity of solids, Meas. Tech. 54 (2012) 1151-1154. https://doi.org/10.1007/s11018-012-9863-8. 


\section{Chapter 6}

[26] E.G. Wolff, D.A. Schneider, Prediction of thermal contact resistance between polished surfaces, Int. J. Heat Mass Transf. 41 (1998) 3469-3482. https://doi.org/10.1016/S00179310(98)00067-2. 
7. Detailed assessment of thermoelectric modules by a comprehensive impedance spectroscopy equivalent circuit 


\title{
Detailed assessment of thermoelectric modules by a comprehensive impedance spectroscopy equivalent circuit
}

\author{
Braulio Beltrán-Pitarch ${ }^{1, *}$, Jesse Maassen ${ }^{2}$, and Jorge García-Cañadas ${ }^{1, *}$
}

${ }^{1}$ Department of Industrial Systems Engineering and Design, Universitat Jaume I, Campus del Riu Sec, 12071 Castellón, Spain

${ }^{2}$ Deparment of Physics and Atmospheric Science, Dalhousie University, 6310 Coburg Rd., Halifax, Canada

*e-mail: beltranb@uji.es, garciaj@uji.es 


\begin{abstract}
Impedance spectroscopy (IS) has been proved to be a useful method for the characterization of thermoelectric (TE) modules. Although impedance models (equivalent circuits) have been recently advanced with the addition of different processes such as convection, radiation, and spreading-constriction, deviations from these models still exist in experimental results, especially in the high frequency part of the impedance spectrum. Here, we present a comprehensive equivalent circuit which covers all the key phenomena that affects the module performance and it is able to explain the observed deviations. The new equivalent circuit includes, as new additions, the thermal influence of the metallic strips (electrodes), combined with the thermal contact resistance between the metallic strips and the outer ceramic layer. The effect of the thermal contact resistance between the TE leg and the electrodes is also analyzed. Moreover, a new more accurate spreading-constriction impedance element, which considers the variation of the heat flow in the radial direction at the outer ceramic surfaces, is also developed. These developments are here shown for both suspended conditions and when modules are contacted by ideal heat sinks. The comprehensive equivalent circuit allowed the qualitative analysis of the impedance response of modules fabricated by different manufacturers that showed significant differences in their response. This opens up the possibility of using IS as a powerful tool to evaluate, monitor, and detect issues in great detail in TE devices.
\end{abstract}




\section{Chapter 7}

\section{Graphical abstract}

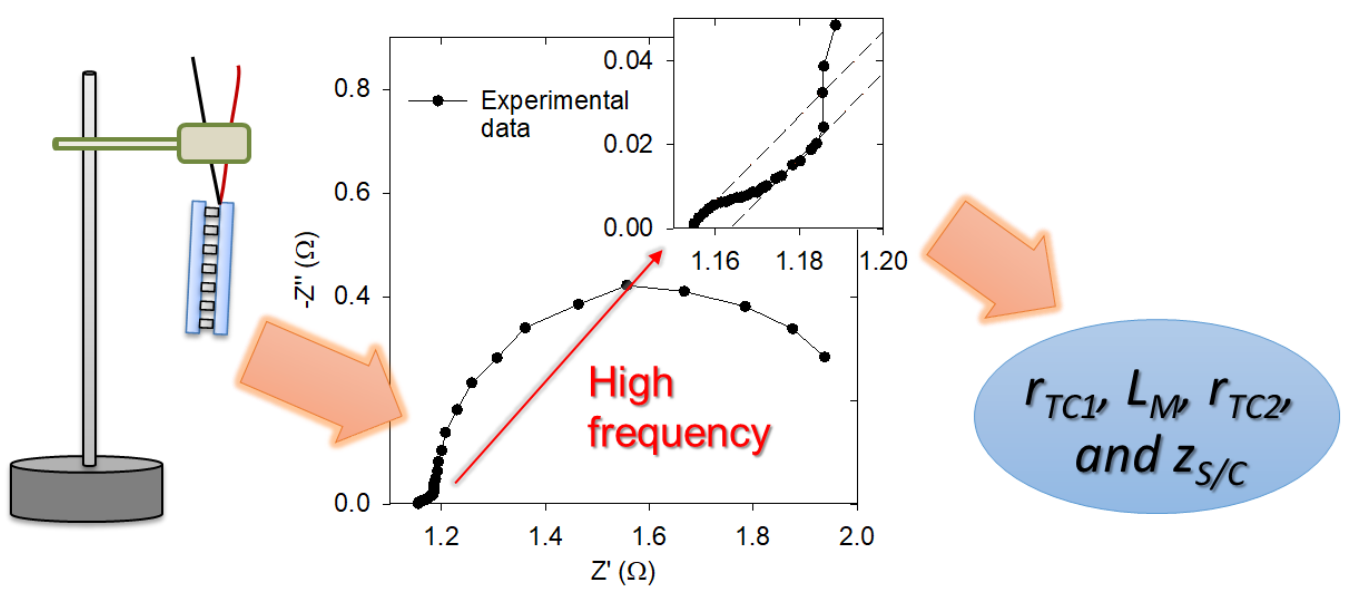

Fig. 7.1. Graphical abstract of the work: Detailed assessment of thermoelectric modules by a comprehensive impedance spectroscopy equivalent circuit. 


\subsection{Introduction}

In recent years, impedance spectroscopy (IS) has been successfully used to measure the dimensionless figure of merit $Z_{m} T$ of thermoelectric (TE) modules [1-4]. In addition, the possibility of performing a complete characterization of TE devices (determination of the internal ohmic resistance, the average Seebeck coefficient and thermal conductivity of the TE legs, and $\left.Z_{m} T\right)$ under suspended conditions if the thermal conductivity of the ceramics is given, has been demonstrated [5-8]. To perform the complete characterization, an ideal equivalent circuit was proposed by us [5], which was obtained by solving the heat equation in the frequency domain. Obtaining the system equivalent circuit is important, since once known, characterization can be performed by fitting the experimental data to the equivalent circuit. In that study, the TE device was thermally modelled by considering multiple TE legs in contact with outer ceramic layers of same cross-sectional area, which led to an equivalent circuit formed by a resistor in series with the parallel combination of constant-temperature and adiabatic Warburg elements.

The ideal model was improved by adding spreading-constriction effects [6,9], which take into account the differences in the cross-sectional area between the TE legs and the outer ceramic layers. Another improvement of the ideal model was obtained by adding the convection effect at the outer ceramic surfaces under non-vacuum conditions [10]. Three new elements in the equivalent circuit were obtained due to this effect. A more comprehensive equivalent circuit included the radiation effect [11] in conjunction with the spreading-constriction and the convection phenomena [6]. Also, it has been included recently the effect of the metallic strips (electrodes), assuming they behave like a capacitor, and the presence of a thermal contact resistance between the thermoelements and the electrodes [12]. Apart from these models under suspended conditions, we have recently reported an impedance equivalent circuit of a module attached to heat sinks at both sides, which takes into account the effect of a thermal contact resistance between the outer ceramic surfaces and the heat sinks [13].

It is worth mentioning that the spreading-constriction impedance was previously reported [6] considering that the heat flux at the outer ceramic surfaces is uniform, which is only true for TE modules suspended in vacuum (without convection) and at room conditions (assuming radiation negligible). An alternative spreading-constriction impedance expression was obtained [9] considering that the temperature at the outer ceramic surfaces is uniform, which only a perfect 
contact with an ideal heat sink could achieve such condition, and this expression also fails to cover intermediate cases.

In this work, we present experimental impedance measurements performed at different TE modules that show features, specially at high frequencies, which cannot be explained by current impedance models. In order to explain these new features, we have developed a new more comprehensive equivalent circuit that includes all the key phenomena affecting the TE module performance. Namely, the effect of the thermal contact resistance between the TE legs and the metallic strips that connect them, the own electrodes contribution, and the thermal contact resistance between the electrodes and the outer ceramic plates. Moreover, a new spreadingconstriction impedance element, which considers the variation of the heat flux in the radial direction at the outer ceramic surfaces, is obtained to cover non-ideal cases. This new equivalent circuit, which also includes convection and radiation effects previously developed, allows the qualitative analysis of the different characteristic features experimentally observed. The fact that IS can identify all these phenomena opens up the possibility of using this method as a quality control tool, able to identify and monitor in detail issues in TE modules.

\subsection{Theoretical model}

In order to obtain an impedance model which includes all the key phenomena that can be relevant in standard TE devices, the model shown in Fig. 7.2 was considered, which consists of a cylindrical TE leg of cross-sectional area $A$ and length $L$, contacted by two metallic strips (usually copper) with a slightly larger cross-sectional area $A / \eta_{M}$ (being $\eta_{M}$ the ratio between the area of all the TE legs and the area of all the metallic strips) and length $L_{M}$, and two external ceramic layers of cross-sectional area $A / \eta$ (being $\eta$ the filling factor of the TE module, i.e. the ratio between the area of all the TE legs and the ceramic area, typically around 0.3), and length $L_{C}$. The use of cylindrical legs has been shown to have no significant differences with respect to a prismatic geometry, and it simplifies the thermal spreading-constriction analysis [14]. At both sides of the metallic strips, the possibility of having a thermal contact resistance is considered, including $r_{T C l}$ and $r_{T C 2}$ as thermal contact resistivities between the TE legs and copper strips, and between the metallic strips and the ceramic layers, respectively. In addition, radiation/convection effects are 
included around the TE legs $\left(h_{0}\right)$, around the metallic strips $\left(h_{1}\right)$, at the inner ceramic surfaces $\left(h_{2}\right)$, and at the outer ceramic surfaces $\left(h_{3}\right)$, as shown in Fig. 7.2 [6,10,11], being,

$$
\begin{aligned}
& h_{0}=h_{i c}+4 \sigma_{B} \varepsilon_{T E} T_{i}^{3} \\
& h_{1}=h_{i c}+4 \sigma_{B} \varepsilon_{M} T_{i}^{3} \\
& h_{2}=h_{i c}+4 \sigma_{B} \varepsilon_{C} T_{i}^{3} \\
& h_{3}=h_{e c}+4 \sigma_{B} \varepsilon_{C} T_{i}^{3}
\end{aligned}
$$

where $\sigma_{B}$ the Stefan-Boltzmann constant, $\varepsilon_{T E}, \varepsilon_{M}$, and $\varepsilon_{C}$ the emissivity of the TE, metallic strip, and external ceramic material, respectively. Moreover, $h_{i c}$ and $h_{e c}$ are the internal and external convection heat transfer coefficients, respectively. It is worth noting that the internal ($L_{M} \leq x \leq L+L_{M}$ ) convection may be negligible in some cases (e.g. large amount of TE legs in the TE module or use of sealants), and the radiation effect depends strongly on temperature.

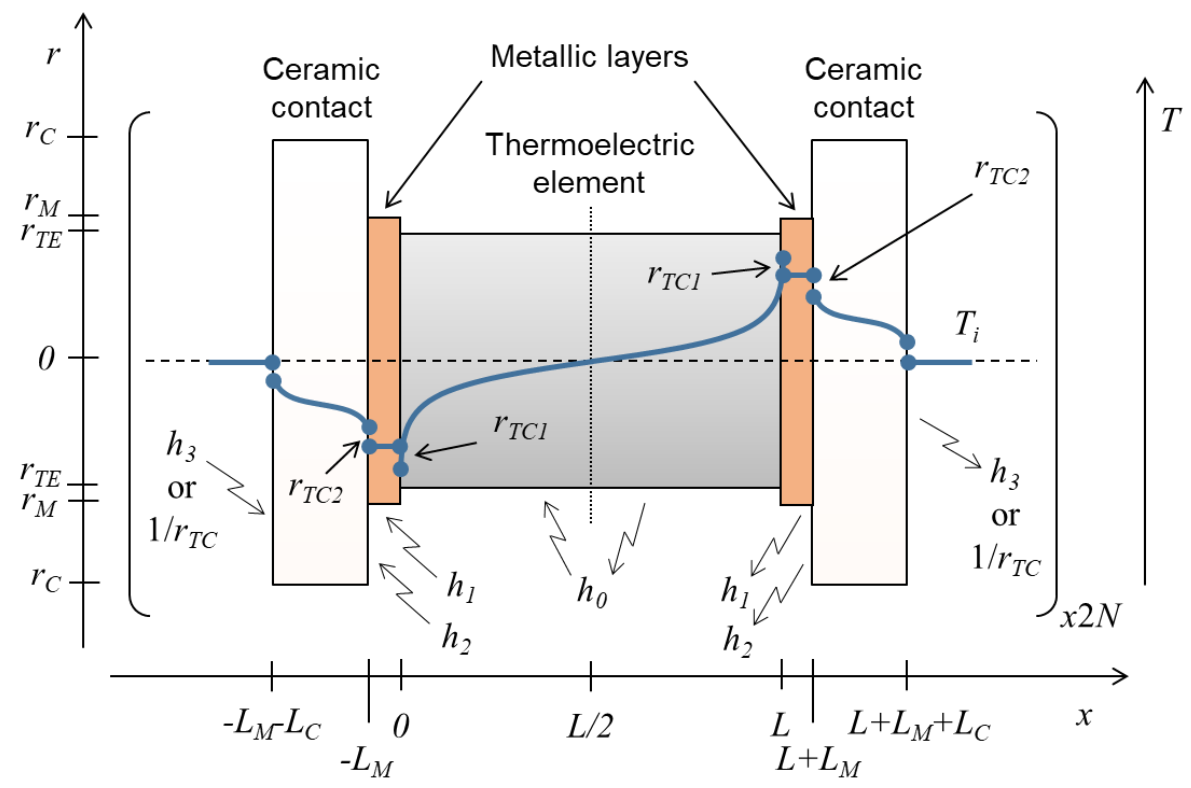

Fig. 7.2. Schematic view of the theoretical model considered in this analysis. A positive value of Seebeck coefficient and current is considered to represent the temperature profile (blue solid lines). The dotted line shows the plane where the temperature remains constant and the dashed line represents the initial temperature profile.

Furthermore, a TE module sandwiched between heat exchangers that do not change their temperature (ideal heat sinks) with a thermal contact resistivity $r_{T C}$ can also be considered at the outer ceramic surfaces instead of convection/radiation, being in this case $h_{3}=1 / r_{T C}$ [13]. Joule 
effect is neglected since the ac amplitude used in IS measurements is small and TE materials usually have relatively high electrical conductivity. It should also be noted that all the TE properties are considered temperature independent, and that the final response must be multiplied by the number of legs, $2 N$ (being $N$ the number of TE couples).

To account for the spreading-constriction impedance introduced at $x=-L_{M}$, and at $x=L+L_{M}$ by the change of the cross-sectional areas, we have developed a new expression considering that the heat released/absorbed at the outer external ceramic surfaces varies radially. It should be noticed that the spreading-constriction at $x=0$ and at $x=L$ can be neglected due to the typically high thermal conductivity of the metallic strips [9]. The new expression was obtained following a similar procedure used by Casalegno et al. [9], with the exception that no constant temperature but convection and radiation at the outer ceramic surfaces was assumed,

$$
\lambda_{C} \frac{\partial \theta_{3}}{\partial x}=-\theta_{3} h_{3}
$$

being $\theta_{3}$ the temperature with respect to the initial temperature $T_{i}$ in the frequency domain $[\theta=\mathscr{L}(T$ $\left.T_{i}\right)$ ] at the outer ceramic surfaces, which may change radially. This change leads to a slightly larger expression for the spreading-constriction impedance (see its derivation in Annex 9.3),

$$
z_{S / c}=\frac{4}{\lambda_{C}} \sum_{n=1}^{\infty} \frac{J_{1}{ }^{2}\left(\delta_{n} \frac{r_{M}}{r_{C}}\right)}{\gamma_{n} \delta_{n}{ }^{2} J_{0}{ }^{2}\left(\delta_{n}\right)}\left[\frac{\gamma_{n} \lambda_{C}+h_{3} \tanh \left(\gamma_{n} L_{C}\right)}{\gamma_{n} \lambda_{C} \tanh \left(\gamma_{n} L_{C}\right)+h_{3}}\right]
$$

being $\lambda_{C}$ the thermal conductivity of the ceramic layer, $J_{0}$ and $J_{l}$ the first kind Bessel functions of order zero and one, respectively, $r_{M}$ and $r_{C}$ the equivalent radii of the metallic strip and ceramic layer, respectively, $\delta_{n}$ is the nth zero of $J_{l}$, and $\gamma_{n}$ is the value for each $\delta_{n}$ that verifies,

$$
\gamma_{n}=\sqrt{\frac{j \omega}{\alpha_{C}}+\left(\frac{\delta_{n}}{r_{C}}\right)^{2}},
$$

where $j=(-1)^{0.5}$ is the imaginary number, $\omega$ the angular frequency ( $\omega=2 \pi f$, being $f$ the frequency), and $\alpha_{C}$ the thermal diffusivity of the external ceramic material.

It is worth highlighting that Eq. (7.6) agrees with the solution obtained for constant heat flux at the outer external ceramic surfaces [6] when the convection/radiation effect is negligible $\left(h_{3} \rightarrow 0\right)$. It also agrees with the solution obtained for constant temperature condition on the same 
surfaces [9] when a perfect contact with a heat exchanger is considered $\left(h_{3} \rightarrow \infty\right)$. Finally, this expression agrees with the solution obtained by Yovanovich et al. [15] for steady state conditions $(\omega \rightarrow 0)$.

The impedance $Z=V / I$ of a suspended TE module is given by,

$$
Z=\frac{V(0)-V(L)}{I_{0}}=R_{\Omega}+2 N \frac{|S|[T(L)-T(0)]}{I_{0}}=R_{\Omega}-2 N \frac{2|S|\left[T(0)-T_{i}\right]}{I_{0}},
$$

where $V(0)$ and $V(L)$ are the voltages at $x=0$ and $x=L$, respectively, $I_{0}$ is the electrical current crossing the device at $x=0, R_{\Omega}$ is the total ohmic resistance of the TE module, which includes the contribution of all the TE legs, the metallic strips, the wires, and the electrical contact resistances, $S$ is the average Seebeck coefficient of the thermoelements, and $T(0)$ and $T(L)$ are the temperatures at $x=0$ and $x=L$, respectively. It is worth noting that in Eq. (7.8) the temperature difference between the thermoelement sides can be determined from the temperature at $x=0$, due to the antisymmetry of the system at $x=L / 2$ (see Fig. 7.2).

To determine $T(0)$ the two-dimensional heat equation in the frequency domain for the three layers (thermoelements, electrodes and ceramics) should be solved, which in cylindrical coordinates takes the form,

$$
\frac{1}{r} \frac{\partial}{\partial r}\left(r \frac{\partial \theta}{\partial r}\right)+\frac{\partial^{2} \theta}{\partial x^{2}}=\frac{j \omega}{\alpha_{i}} \theta
$$

where $r$ and $x$ are the radial and axial axes (shown in Fig. 7.2), respectively, and $\alpha_{i}$ the average thermal diffusivity of each material: TE legs $(i=T E)$, metallic strips $(i=M)$, and ceramic layers $(i=C)$. However, averaging the temperature distribution over the equivalent circular surface $(\bar{\theta})$ of radius $r_{k}$ (being $k=0$ for the TE legs and the metallic layers, and $k=1$ for the ceramic layers),

$$
\bar{\theta}(x, j \omega)=\frac{2}{r_{k}^{2}} \int_{0}^{r_{k}} r \theta(r, x, j \omega) \partial r,
$$

the two-dimensional heat equation given in Eq. (7.9) can be approximated to a one-dimensional heat equation [16],

$$
\frac{\partial^{2} \bar{\theta}}{\partial x^{2}}-\left(\frac{2 h_{i}}{\lambda_{i} r_{k}}+\frac{j \omega}{\alpha_{i}}\right) \bar{\theta}=0,
$$


being $\lambda_{i}$ the average thermal conductivity of each material, TE legs $(i=T E)$, metallic strips $(i=M)$, and ceramic layers $(i=C)$, and $h_{i}$ the convection/radiation effects around the TE legs $\left(h_{0}\right)$, and around the metallic strips $\left(h_{l}\right)$. Notice that the convection/radiation from the edges of the ceramics can be neglected and hence the term $2 h_{i} /\left(\lambda_{i} r_{k}\right)$ is not included for this layer (see Fig. 7.2).

We can now make use of the definition of the total thermal admittance $y_{0}=\phi_{0} / \theta_{0}$, being $\phi_{0}$ the heat power at $x=0$ and $\theta_{0}=\theta(x=0)$ at the TE leg side. The total thermal admittance, is the series summation of the thermal admittance towards the TE element, $y_{T E}$, and towards the metallic strip, $y_{e}[2]$,

$$
\frac{1}{y_{0}}=\frac{1}{y_{T E}+y_{e}}
$$

Since the heat power at $x=0$ is the Peltier heat [due to the small ac current amplitude applied, it is approximately $\phi_{0}=-|S| T_{i} i_{0}$, being $\left.i_{0}=\mathscr{L}\left(I_{0}\right)\right]$, Eq. (7.8) can be rewritten in the frequency domain as,

$$
Z=R_{\Omega}+\frac{4 N|S| \theta_{0}}{i_{0}}=R_{\Omega}+\frac{4 N|S| \phi_{0}}{i_{0} y_{0}}=R_{\Omega}+\frac{4 N S^{2} T_{i}}{y_{0}}
$$

For the determination of $y_{T E}$ and $y_{e}$, the thermal quadrupole method was used [16]. In this method, the different equations are displayed in different matrices, where each matrix represents one layer (TE material, metal strip, or ceramic) or one thermal restriction (thermal contact resistances, spreading-constriction impedance, and convection/radiation effects). The matrix that defines the TE elements admittance $\left(1 / y_{T E}=\theta_{0, T E} / \phi_{0, T E}\right)$ is only considered until the half length of the TE layer, since a boundary condition at this position $(x=L / 2)$ exists due to the anti-symmetry $[\theta(L / 2)=0]$,

$$
\begin{aligned}
& {\left[\begin{array}{l}
\theta_{0, T E} \\
\phi_{0, T E}
\end{array}\right]} \\
& =\left[\begin{array}{cc}
\cosh \left(\sqrt{\left.\frac{h_{0} L}{2 \lambda_{T E} r_{T E}}+\frac{j \omega}{\omega_{T E}}\right)}\right. & \frac{L \sinh \left(\sqrt{\frac{h_{0} L}{2 \lambda_{T E} r_{T E}}+\frac{j \omega}{\omega_{T E}}}\right)}{2 \lambda_{T E} A \sqrt{\frac{h_{0} L}{2 \lambda_{T E} r_{T E}}+\frac{j \omega}{\omega_{T E}}}} \\
\frac{2 \lambda_{T E} A}{L} \sqrt{\frac{h_{0} L}{2 \lambda_{T E} r_{T E}}+\frac{j \omega}{\omega_{T E}}} \sinh \left(\sqrt{\frac{h_{0} L}{2 \lambda_{T E} r_{T E}}+\frac{j \omega}{\omega_{T E}}}\right) & \cosh \left(\sqrt{\frac{h_{0} L}{2 \lambda_{T E} r_{T E}}+\frac{j \omega}{\omega_{T E}}}\right)
\end{array}\right]\left[\begin{array}{c}
0 \\
\phi_{L / 2}
\end{array}\right]
\end{aligned}
$$


where $\omega_{T E}$ is the characteristic angular frequency $\left[\omega_{T E}=\alpha_{T E} /(L / 2)^{2}\right]$, being $\alpha_{T E}$ the average thermal diffusivity of the TE legs, $\lambda_{T E}$ the average thermal conductivity of the TE legs, and $\phi_{L / 2}$ the heat flow in the frequency domain at $x=L / 2$.

The matrix that defines the heat flow going towards the metallic strip $\left(1 / y_{e}=\theta_{0, e} / \phi_{0, e}\right)$ is given by seven matrices, which must be written in a proper order. The first matrix corresponds to the thermal contact resistance between the TE layer and the metallic strip. The second matrix corresponds to the metallic strip itself. The third one to the thermal contact resistance between the metallic strip and the ceramic layer. The fourth matrix is the spreading-constriction impedance of the heat entering the ceramic (which has a larger area than the metallic strip). The fifth one denotes the heat lost in the inner part of the ceramic due to convection/radiation. It should be noticed that in this matrix the area of ceramic considered to be exposed to inner convection is slightly higher than it should, since it also includes the exposed part of the metal $\left(r_{M^{-}} r_{T E}\right)$, which is adopted for simplicity. The sixth one relates to the ceramic layer. Finally, the seventh matrix corresponds to the boundary condition at $x=-L_{M}-L_{C}$, which describes the convection/radiation at the outer ceramic surfaces $\left(\phi_{3}=\theta_{3} h_{3} A / \eta\right)$,

$$
\begin{aligned}
& {\left[\begin{array}{l}
\theta_{0, e} \\
\phi_{0, e}
\end{array}\right]} \\
& =\left[\begin{array}{cc}
1 & \frac{r_{T C 1}}{A} \\
0 & 1
\end{array}\right]\left[\begin{array}{cc}
\cosh \left(\sqrt{\frac{2 h_{1} L_{M}}{\lambda_{M} r_{M}}+\frac{j \omega}{\omega_{M}}}\right) & \frac{L_{M} \eta_{M} \sinh \left(\sqrt{\frac{2 h_{1} L_{M}}{\lambda_{M} r_{M}}+\frac{j \omega}{\omega_{M}}}\right)}{\lambda_{M} A \sqrt{\frac{2 h_{1} L_{M}}{\lambda_{M} r_{M}}+\frac{j \omega}{\omega_{M}}}} \\
\frac{\lambda_{M} A}{L_{M} \eta_{M}} \sqrt{\frac{2 h_{1} L_{M}}{\lambda_{M} r_{M}}+\frac{j \omega}{\omega_{M}}} \sinh \left(\sqrt{\frac{2 h_{1} L_{M}}{\lambda_{M} r_{M}}+\frac{j \omega}{\omega_{M}}}\right) & \cosh \left(\sqrt{\frac{2 h_{1} L_{M}}{\lambda_{M} r_{M}}+\frac{j \omega}{\omega_{M}}}\right)
\end{array}\right] \\
& {\left[\begin{array}{cc}
1 & \frac{r_{T C 2} \eta_{M}}{A} \\
0 & 1
\end{array}\right]\left[\begin{array}{cc}
1 & \frac{z_{S / c} \eta_{M}}{A} \\
0 & 1
\end{array}\right]\left[\begin{array}{cc}
1 & 0 \\
h_{2} A\left(\frac{1-\eta}{\eta}\right) & 1
\end{array}\right]} \\
& {\left[\begin{array}{cc}
\cosh \left(\sqrt{\frac{j \omega}{\omega_{C}}}\right) & \frac{L_{C} \eta \sinh \left(\sqrt{\frac{j \omega}{\omega_{C}}}\right)}{\lambda_{C} A \sqrt{\frac{j \omega}{\omega_{C}}}} \\
\frac{\lambda_{C} A}{L_{C} \eta} \sqrt{\frac{j \omega}{\omega_{C}}} \sinh \left(\sqrt{\frac{j \omega}{\omega_{C}}}\right) & \cosh \left(\sqrt{\frac{j \omega}{\omega_{C}}}\right)
\end{array}\right]\left[\begin{array}{c}
\theta_{3} \\
\frac{h_{3} A}{\eta} \theta_{3}
\end{array}\right],}
\end{aligned}
$$


where $\omega_{M}=\alpha_{M} / L_{M}^{2}$ (being $\alpha_{M}$ the thermal diffusivity of the metallic layers), $\omega_{C}=\alpha_{C} / L_{C}{ }^{2}, \lambda_{M}$ and $\lambda_{C}$ are the characteristic angular frequencies and thermal conductivities of the metallic strips and ceramic layers, respectively.

Once the two admittances are defined, they are introduced in Eq. (7.13) using Eq. (7.12), to obtain the impedance function. After some algebraic steps, the equivalent circuit shown in Fig. 7.3 is obtained and takes the shape,

$$
\begin{aligned}
Z=R_{\Omega}+\left\{Z_{W C T}{ }^{-1}\right. & \\
+ & {\left[R_{T C 1}+\left(Z_{W a, M}{ }^{-1}+Z_{T O T 1}{ }^{-1}\right)^{-1}\right.} \\
& \left.\left.+\left(Z_{W C T, M}{ }^{-1}+Z_{T O T 2}{ }^{-1}\right)^{-1}\right]^{-1}\right\}^{-1}
\end{aligned}
$$

being $Z_{\text {TOTI }}$ and $Z_{\text {TOT2, }}$

$$
\begin{aligned}
Z_{\text {TOT } 1}=R_{T C 2}+ & Z_{S / C} \\
& +\left\{R_{h 2}{ }^{-1}\right. \\
& \left.+\left[\left(Z_{W a}{ }^{-1}+R_{h 3}{ }^{-1}\right)^{-1}+\left(Z_{W C T, C}{ }^{-1}+Z_{C_{h 3}}{ }^{-1}\right)^{-1}\right]^{-1}\right\}^{-1}, \\
Z_{\text {TOT2 }}{ }^{-1}=Z_{C_{T C 2}}{ }^{-1}+Z_{S / C, M}{ }^{-1} & +\left\{Z_{C_{h 2}}\right. \\
& \left.+\left[\left(Z_{W C T, C, M}+Z_{C_{h 3, M}}\right)^{-1}+\left(Z_{W a, C, M}+R_{h 3, M}\right)^{-1}\right]^{-1}\right\}^{-1} .
\end{aligned}
$$

The elements in Eqs. (7.16), (7.17), and (7.18) are defined by,

$$
\begin{aligned}
Z_{W C T}= & \frac{2 N S^{2} T_{i} L}{\lambda_{T E} A}\left(\frac{h_{0} L}{2 \lambda_{T E} r_{T E}}+\frac{j \omega}{\omega_{T E}}\right)^{-0.5} \tanh \left[\left(\frac{h_{0} L}{2 \lambda_{T E} r_{T E}}+\frac{j \omega}{\omega_{T E}}\right)^{0.5}\right] \\
= & R_{T E}\left(\frac{h_{0} L}{2 \lambda_{T E} r_{T E}}+\frac{j \omega}{\omega_{T E}}\right)^{-0.5} \tanh \left[\left(\frac{h_{0} L}{2 \lambda_{T E} r_{T E}}+\frac{j \omega}{\omega_{T E}}\right)^{0.5}\right], \\
R_{T C 1}= & \frac{4 N S^{2} T_{i} r_{T C 1}}{A}
\end{aligned}
$$




$$
\begin{gathered}
Z_{W a, M}=\frac{4 N S^{2} T_{i} L_{M} \eta_{M}}{\lambda_{M} A}\left(\frac{2 h_{1} L_{M}}{\lambda_{M} r_{M}}+\frac{j \omega}{\omega_{M}}\right)^{-0.5} \operatorname{coth}\left[\left(\frac{2 h_{1} L_{M}}{\lambda_{M} r_{M}}+\frac{j \omega}{\omega_{M}}\right)^{0.5}\right] \\
=R_{M}\left(\frac{2 h_{1} L_{M}}{\lambda_{M} r_{M}}+\frac{j \omega}{\omega_{M}}\right)^{-0.5} \operatorname{coth}\left[\left(\frac{2 h_{1} L_{M}}{\lambda_{M} r_{M}}+\frac{j \omega}{\omega_{M}}\right)^{0.5}\right], \\
Z_{W C T, M}=\frac{4 N S^{2} T_{i} L_{M} \eta_{M}}{\lambda_{M} A}\left(\frac{2 h_{1} L_{M}}{\lambda_{M} r_{M}}+\frac{j \omega}{\omega_{M}}\right)^{-0.5} \tanh \left[\left(\frac{2 h_{1} L_{M}}{\lambda_{M} r_{M}}+\frac{j \omega}{\omega_{M}}\right)^{0.5}\right] \\
=R_{M}\left(\frac{2 h_{1} L_{M}}{\lambda_{M} r_{M}}+\frac{j \omega}{\omega_{M}}\right)^{-0.5} \tanh \left[\left(\frac{2 h_{1} L_{M}}{\lambda_{M} r_{M}}+\frac{j \omega}{\omega_{M}}\right)^{0.5}\right],
\end{gathered}
$$

$Z_{W a}=\frac{4 N S^{2} T_{i} L_{C} \eta}{\lambda_{C} A}\left(\frac{j \omega}{\omega_{C}}\right)^{-0.5} \operatorname{coth}\left[\left(\frac{j \omega}{\omega_{C}}\right)^{0.5}\right]=R_{C}\left(\frac{j \omega}{\omega_{C}}\right)^{-0.5} \operatorname{coth}\left[\left(\frac{j \omega}{\omega_{C}}\right)^{0.5}\right]$,

$$
R_{h 3}=\frac{4 N S^{2} T_{i} \eta}{h_{3} A}
$$

$Z_{W C T, C}=\frac{4 N S^{2} T_{i} L_{C} \eta}{\lambda_{C} A}\left(\frac{j \omega}{\omega_{C}}\right)^{-0.5} \tanh \left[\left(\frac{j \omega}{\omega_{C}}\right)^{0.5}\right]=R_{C}\left(\frac{j \omega}{\omega_{C}}\right)^{-0.5} \tanh \left[\left(\frac{j \omega}{\omega_{C}}\right)^{0.5}\right]$,

$Z_{C_{h 3}}=\frac{4 N S^{2} T_{i} L_{C}{ }^{2} h_{3} \eta}{\lambda_{C}{ }^{2} A}\left(\frac{j \omega}{\omega_{C}}\right)^{-1}=\frac{R_{C}{ }^{2}}{R_{h 3}}\left(\frac{j \omega}{\omega_{C}}\right)^{-1}=\frac{1}{j \omega C_{h 3}}$

$Z_{C_{T C 2}}=\frac{4 N S^{2} T_{i} L_{M}{ }^{2} \eta_{M}}{\lambda_{M}{ }^{2} A r_{T C 2}}\left(\frac{j \omega}{\omega_{M}}\right)^{-1}=\frac{R_{M}{ }^{2}}{R_{T C 2}}\left(\frac{j \omega}{\omega_{M}}\right)^{-1}=\frac{1}{j \omega C_{T C 2}}$,

$Z_{S / C, M}=\frac{4 N S^{2} T_{i} L_{M}{ }^{2} \eta_{M}}{\lambda_{M}{ }^{2} A z_{S / C}}\left(\frac{j \omega}{\omega_{M}}\right)^{-1}=\frac{R_{M}{ }^{2}}{Z_{S / C}}\left(\frac{j \omega}{\omega_{M}}\right)^{-1}$,

$Z_{C_{h 2}}=\frac{4 N S^{2} T_{i} L_{M}{ }^{2} h_{2}(1-\eta) \eta_{M}{ }^{2}}{\lambda_{M}{ }^{2} A \eta}\left(\frac{j \omega}{\omega_{M}}\right)^{-1}=\frac{R_{M}{ }^{2}}{R_{h 2}}\left(\frac{j \omega}{\omega_{M}}\right)^{-1}=\frac{1}{j \omega C_{h 2}}$, 


$$
\begin{gathered}
Z_{W C T, C, M}=\frac{4 N S^{2} T_{i} L_{M}{ }^{2} \lambda_{C} \omega_{M} \eta_{M}{ }^{2}}{\lambda_{M}{ }^{2} A L_{C} \eta \omega_{C}}\left(\frac{j \omega}{\omega_{C}}\right)^{-0.5} \tanh \left[\left(\frac{j \omega}{\omega_{C}}\right)^{0.5}\right] \\
=\frac{R_{M}{ }^{2}}{R_{C}}\left(\frac{\omega_{M}}{\omega_{C}}\right)\left(\frac{j \omega}{\omega_{C}}\right)^{-0.5} \tanh \left[\left(\frac{j \omega}{\omega_{C}}\right)^{0.5}\right], \\
Z_{C_{h 3, M}}=\frac{4 N S^{2} T_{i} L_{M}{ }^{2} h_{3} \eta_{M}{ }^{2}}{\lambda_{M}{ }^{2} A \eta}\left(\frac{j \omega}{\omega_{M}}\right)^{-1}=\frac{R_{M}{ }^{2}}{R_{h 3}}\left(\frac{j \omega}{\omega_{M}}\right)^{-1}=\frac{1}{j \omega C_{h 3, M}}, \\
Z_{W a, C, M}=\frac{4 N S^{2} T_{i} L_{M}{ }^{2} \lambda_{C} \omega_{M} \eta_{M}{ }^{2}}{\lambda_{M}{ }^{2} A L_{C} \eta \omega_{C}}\left(\frac{j \omega}{\omega_{C}}\right)^{-0.5} \operatorname{coth}\left[\left(\frac{j \omega}{\omega_{C}}\right)^{0.5}\right] \\
=\frac{R_{M}{ }^{2}}{R_{C}}\left(\frac{\omega_{M}}{\omega_{C}}\right)\left(\frac{j \omega}{\omega_{C}}\right)^{-0.5} \operatorname{coth}\left[\left(\frac{j \omega}{\omega_{C}}\right)^{0.5}\right], \\
R_{h 3, M}=\frac{4 N S^{2} T_{i} L_{M}{ }^{2} \lambda_{C}{ }^{2} \omega_{M} \eta_{M}{ }^{2}}{\lambda_{M}{ }^{2} A L_{C}{ }^{2} h_{3} \eta \omega_{C}}=\frac{R_{M}{ }^{2} R_{h 3}}{R_{C}{ }^{2}}\left(\frac{\omega_{M}}{\omega_{C}}\right) .
\end{gathered}
$$

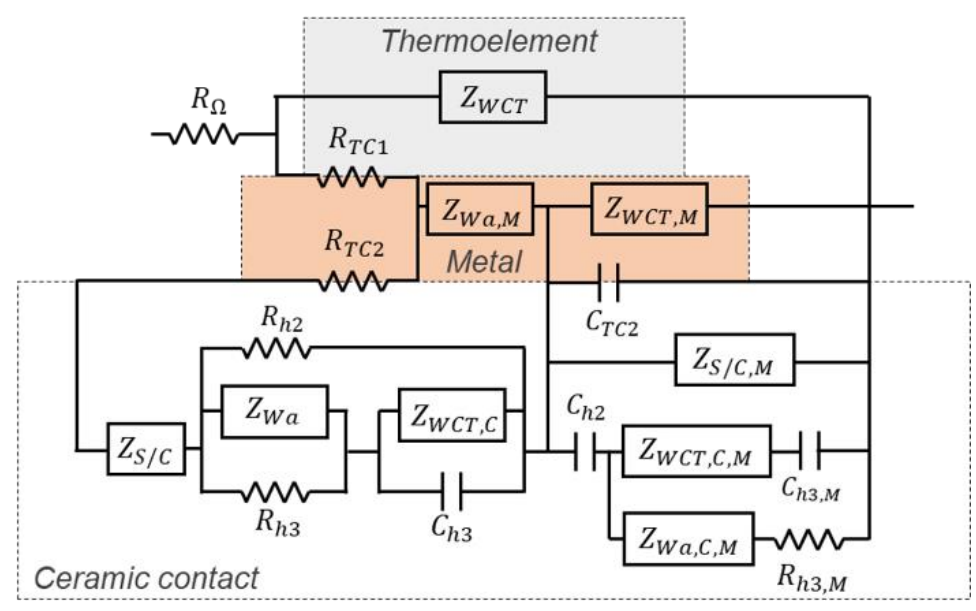

Fig. 7.3. Comprehensive equivalent circuit with all the key phenomena that can occur in thermoelectric devices.

This equivalent circuit contains the total ohmic resistance of the TE device $\left(R_{\Omega}\right)$, the constanttemperature Warburg element $\left(Z_{W C T}\right)$ due to the TE legs, and the adiabatic Warburg element $\left(Z_{W a}\right)$ due to the external ceramics, which are the three elements of the ideal model [5]. It also contains the resistance $R_{h 3}=4 N S^{2} T_{i} \eta /\left(h_{3} A\right)$, the constant-temperature Warburg impedance $Z_{W C T, C}$, and the capacitor $C_{h 3}=R_{h 3} /\left(R_{C}^{2} \omega_{C}\right)$. These 3 elements appear when heat is exchanged at the outer ceramic 
surfaces of the TE module (either by convection/radiation in suspended modules, or by the effect of heat sinks when they are contacted to the module) $[6,10,13]$.

The resistance $R_{h 2}=4 N S^{2} T_{i} \eta /\left[h_{2} A(1-\eta)\right]$, due to the heat removal on the inner ceramic surfaces, and the spreading-constriction impedance $Z_{S / C}$, due to the effect of the area variation between the TE legs and the ceramics, are also present, as previously reported [12]. However, it should be noted that the position of $R_{h 2}$ identified in the equivalent circuit in our analysis [in parallel with the combination of the four elements of the ceramics $\left(Z_{W a}, R_{h 3}, Z_{W C T, C}\right.$, and $\left.C_{h 3}\right)$ and in series with $Z_{S / C}$, see Fig. 7.3] is different from that shown in [12], where it is in parallel with $Z_{S / C}$. This is due to a different position adopted here for the matrix of the spreading-constriction [between the $z_{s / c}$ and the external ceramic matrices, see Eq. (7.15)] which we think it is more correct, since the heat losses from the internal surface of the ceramics is not possible if the heat flow is not spread. In any case, it is expected that this modification will not produce large deviations for commercial TE modules in suspended conditions, since the heat removal by the internal convection and radiation effects is low and the thermal conductivity of the ceramics is high.

The resistance $R_{T C l}=4 N S^{2} T_{i} r_{T C l} / A$, due to the presence of a thermal contact resistance between the TE legs and the metallic strips, is also part of the equivalent circuit of Fig. 7.3. When this thermal contact exists, part of the heat flowing towards the ceramic is blocked. If this thermal contact is very large $\left(R_{T C l} \rightarrow \infty\right)$, the heat only flows towards the TE legs, and only the $Z_{W C T}$ element (in series with $R_{\Omega}$ ) is observed. On the other hand, if this thermal contact is negligible, $R_{T C l}=0$, and this element can be replaced by a short circuit (graphically it will be represented by a wire in Fig. 7.3). The resistance $R_{T C l}$ and $Z_{W a, M}$, which can become a capacitor for metallic strips of high thermal conductivity, as it is the case for copper [17], were previously identified when the effect of the metallic strips was taken into account in the impedance response [12].

In addition to all the aforementioned elements, nine new elements appear: two resistances $\left(R_{T C 2}\right.$ and $\left.R_{h 3, M}\right)$, one adiabatic Warburg element $\left(Z_{W a, C, M}\right)$, two constant-temperature Warburg elements $\left(Z_{W C T, M}\right.$ and $\left.Z_{W C T, C, M}\right)$, a new expression for the spreading-constriction impedance $\left(Z_{S / C, M}\right)$, and three capacitors $\left[C_{T C 2}=R_{T C 2} /\left(R_{M}^{2} \omega_{M}\right), C_{h 2}=R_{h 2} /\left(R_{M}{ }^{2} \omega_{M}\right)\right.$, and $\left.C_{h 3, M}=R_{h 3} /\left(R_{M}^{2} \omega_{M}\right)\right]$.

The resistance $R_{T C 2}=4 N S^{2} T_{i} r_{T C 2} \eta_{M} / A$ comes from the thermal contact resistance between the metallic strips and the ceramic layers, and it appears twice in the equivalent circuit since it is also 
present in the denominator of the capacitor $C_{T C 2}$ [see Eq. (7.30)]. $R_{T C 2}=0$ and becomes a short circuit if the thermal contact is negligible, but for a large thermal contact resistivity $\left(R_{T C 2} \rightarrow \infty\right)$, it blocks all the heat flow towards the ceramic elements, leaving the contribution to the equivalent circuit of both the metallic strips and the external ceramics with only the adiabatic Warburg element of the metallic strips $\left(Z_{W a, M}\right)$ in series with $R_{T C l}$, since $Z_{C T C 2} \rightarrow 0$. A more detailed information about the physical meaning of resistors and capacitors that come from the existence of a thermal contact resistance can be found in ref. [13].

The elements $Z_{W a, M}$, and $Z_{W C T, M}$ appear in the equivalent circuit due to the metallic strips, as it was the case in our previously published article when we added the effect of the convection at the outer ceramic surfaces to the ideal model [10]. The element $C_{h 2}=\lambda_{M} d_{M} C_{p, M} A \eta /\left[4 N S^{2} T_{\text {initial }} \eta h_{2}(1-\right.$

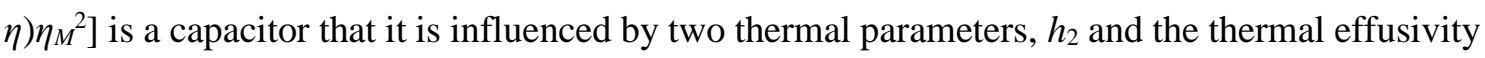
of the metallic strips $e_{M}=\left(\lambda_{M} d_{M} C_{p, M}\right)^{0.5}$, Both $h_{2}$ and $e_{M}$ determine the temperature of the junction at the side of the metallic layer, which is eventually governed by the heat release/accumulation at the interface [13]. If the metallic strips are large, the accumulation of heat in $C_{h 2}$ will be high but, that accumulation will be lower when heat losses through the internal surface of the ceramics increases (higher $h_{2}$ ). The elements $Z_{S / C, M}, Z_{W C T, C, M}, C_{h 3, M}, Z_{W a, C, M}$, and $R_{h 3, M}$ appear in the equivalent circuit when the influence of the metallic strips cannot be neglected. It should eb noticed that all these elements involve the properties of the metallic strips, and they disappear if the metallic layers are not present $\left(L_{M}=0\right)$.

\subsection{Analysis of equivalent circuits}

To study the influence of all the elements in the new equivalent circuit, impedance spectroscopy simulations from $10 \mathrm{mHz}$ to $1 \mathrm{MHz}$ were performed systematically using different elements and varying parameters such as $\eta, r_{T C l}, r_{T C 2}$, and $L_{M}$. For all the simulations $R_{\Omega}=0$, and $\eta_{M}=1$ were considered for simplicity, and typical values of a commercial Bi-Te TE module were used $\left(N=127, S=190 \mu \mathrm{VK}^{-1}, \lambda_{T E}=1.5 \mathrm{Wm}^{-1} \mathrm{~K}^{-1}, \alpha_{T E}=0.37 \mathrm{~mm}^{2} \mathrm{~s}^{-1}, L=1.64 \mathrm{~mm}, \lambda_{C}=25 \mathrm{Wm}^{-1} \mathrm{~K}^{-1}\right.$, $\alpha_{C}=10 \mathrm{~mm}^{2} \mathrm{~s}^{-1}, L_{C}=0.57 \mathrm{~mm}, \lambda_{M}=400 \mathrm{Wm}^{-1} \mathrm{~K}^{-1}, \alpha_{M}=110 \mathrm{~mm}^{2} \mathrm{~s}^{-1}, A=1.69 \mathrm{~mm}^{2}$, and $\left.T_{\text {initial }}=300 \mathrm{~K}\right)$. Also, all the internal convection/radiation losses were discarded $\left(h_{0}=0, h_{l}=0\right.$, and $\left.h_{2}=0\right)$. Simulations for modules suspended and also contacted (sandwiched) by ideal heat sinks are considered. The latter is the case when modules are characterized using a heat source whose 
temperature is kept fixed by the action of a temperature controller. For the simulations of modules in suspended conditions, adiabatic conditions were considered $\left(h_{3}=0\right)$. For modules contacted, a thermal contact resistivity $r_{T C}=1 / h_{3}=10^{-4} \mathrm{~m}^{2} \mathrm{KW}^{-1}$ is adopted for the heat sink/external ceramic thermal contact.

\subsubsection{Thermal influence of metallic strips neglected $\left(L_{M} \rightarrow 0\right)$. Evaluation of the effect of the thermal contact resistance and $Z_{S / C}$}

Fig. 7.4 shows the IS simulations for different values of $\eta$ and $r_{T C l}$ when $L_{M}=0$ and $r_{T C 2}=0$. In this case, the thermal influence of the metallic strips is neglected and the equivalent circuit of Fig. 7.3 reduces to the circuits shown in Fig. 7.5. It should be noted that in this case $r_{T C l}$ provides the combination of both TE/metallic strip and metallic strip/ceramic thermal contact resistances, since although the metallic strips are thermally considered extremely thin, they are always present in a module, thus, both thermal contacts exist.

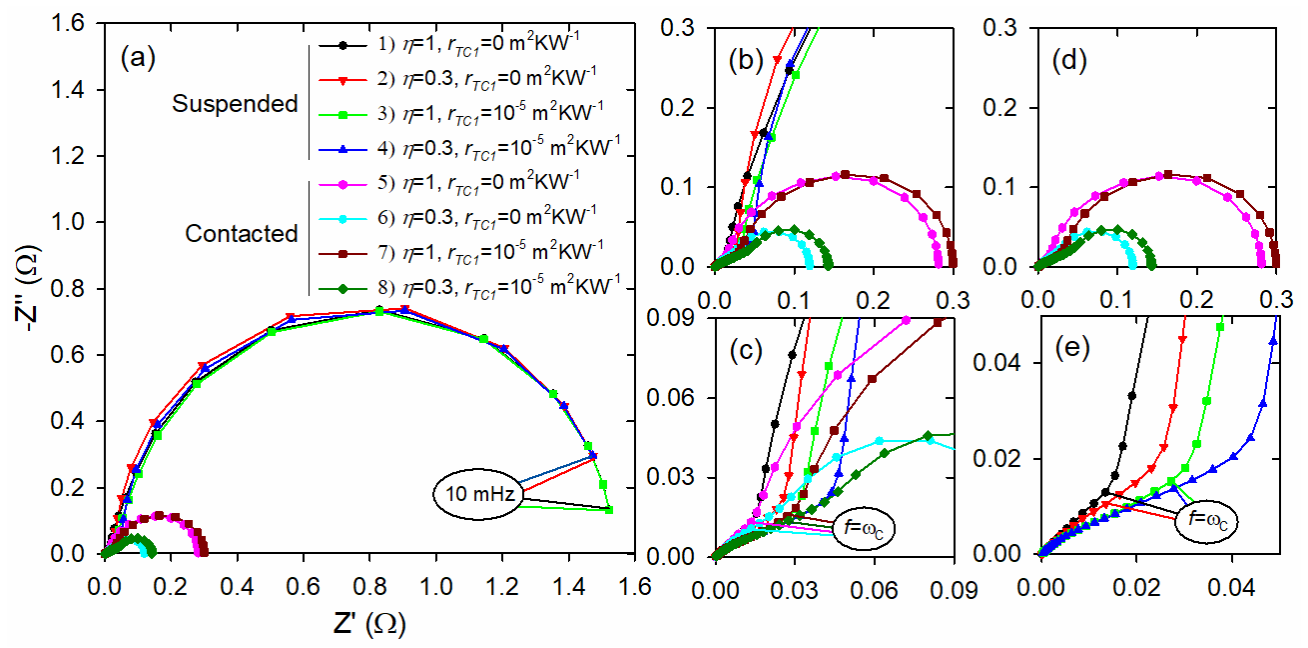

Fig. 7.4. Impedance spectroscopy simulations when $L_{M}=0$ for different values of the filling factor $\eta$, and thermal contact resistivity $r_{T C I}$. The four larger spectra simulations consider a module suspended in vacuum, and the other four consider it in contact with ideal heat sinks with a thermal contact resistivity $r_{T C}=1 / h_{3}=10^{-}$ ${ }^{4} \mathrm{~m}^{2} \mathrm{KW}^{-1}$. Plots (b) and (c) are magnifications of the bottom left part in (a) (same axis units). Plots (d) and (e) are magnifications for the suspended and contacted configurations, respectively.

The four larger spectra in Fig. 7.4 consider the case of a module suspended in vacuum (equivalent circuit of Fig. 7.5b), and the four smaller ones consider the module contacted with ideal heat sinks assuming a thermal contact resistivity $r_{T C}=1 / h_{3}=10^{-4} \mathrm{~m}^{2} \mathrm{KW}^{-1}$ (equivalent circuit of Fig. 7.5a). It should be noticed that the elements $Z_{W C T, C}$ and $C_{h 3}$ can be neglected for suspended standard commercial modules under normal conditions [10]. In Fig. 7.4, a significant reduction 
in the impedance response is observed for the contacted modules, as a result of the contact with the heat sinks with a non-negligible thermal contact resistance, as previously discussed in ref. [13]. It should be also noticed, that in contrast with the significant changes mentioned, which occur at low frequencies (right part of the spectra), the impedance response is basically the same at high frequencies (left part of the spectra) as shown in Fig. 7.4c, since no differences occur between the two cases (suspended and contacted) until the outer surfaces of the external ceramics are reached. This is a common circumstance that can be extended to all the analysis performed in this section.
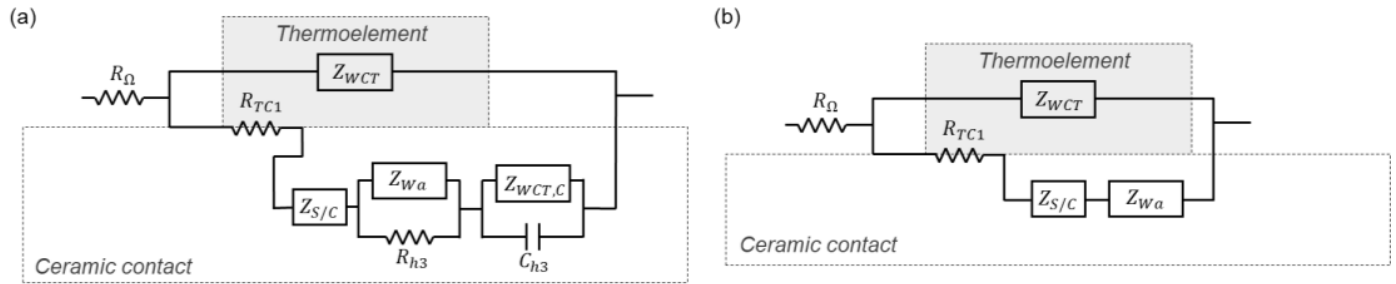

Fig. 7.5. Simplified equivalent circuits of thermoelectric modules when $h_{2}=0, L_{M}=0$, and $r_{T C 2}=0$ for (a) contacted modules and (b) suspended modules in vacuum $\left(h_{3}=0\right)$.

A first comparison can be made in Fig. 7.4 between cases 1 and 2, where $r_{T C l}$ is neglected and the main difference is the filling factor $\eta$. When $\eta=1$, it should be noticed that the spreadingconstriction effect disappears $\left[z_{s / C}=0\right.$, see Eq. (7.6) $]$ and the response of case 1 is the same as the ideal equivalent circuit [5], i.e. a $45^{\circ}$ straight line at high frequencies that turns into a semicircle for $f<\omega_{c}$. When spreading-constriction effects are present (case 2), the $45^{\circ}$ straight line reduces slightly its slope when it gets closer to its termination. Also, the straight line becomes more prominent (see Fig. 7.4e). Moreover, the point where $f=\omega_{C}$ becomes located at higher frequencies than the point where the straight line terminates. This delay effect in the response, caused by the spreading-constriction of the heat flow, is also observed at the lowest frequency simulated (10 $\mathrm{mHz}$ ) in Fig. 7.4a for the cases 2 and 4, which become further from the real axis than the cases where $\eta=1$.

A second comparison can be made between cases 1 and 3 of Fig. 7.4 to evaluate the effect of the presence of $r_{T C l}$ when spreading-constriction effects do not exist. It can be observed that the variations between these two cases are quite similar to the previous comparison, i.e. the $45^{\circ}$ straight line reduces slightly its slope when it gets closer to its end, and becomes more prominent (see Fig. 7.4e). However, in the comparison between cases 1 and 3 the point where $f=\omega_{C}$ is located 
matches the termination of the straight line (there is no delay), as shown in Fig. 7.4e. Also, the last simulated frequency $(10 \mathrm{mHz})$ locates at the same place for both simulations (see Fig. 7.4a). This is an important difference that may serve to distinguish between these two effects (spreadingconstriction and thermal contact resistance) when metallic strips can be thermally neglected.

To evaluate the effect of the presence of $r_{T C l}$ in standard modules, where the spreadingconstriction effect is always present, since $\eta \approx 0.3$, a third comparison involving cases 2 and 4 can be made. As expected from the previous analysis, in both cases the delay $\left(f=\omega_{C}\right.$ located at higher frequencies than the straight line termination) is observed due to the existence of $Z_{S / C}$. It is also evident from Fig. 7.4e that the presence of the thermal contact resistance reduces more significantly the slope of the linear high frequency part. Moreover, it also makes this part more prominent in the spectra.

For the case of TE modules contacted by heat sinks, the same analysis performed above applies for the high frequency part, since this part overlaps with the suspended cases as previously mentioned. However, at low frequencies, significant differences appear. First, a significant reduction of the semicircle can be observed when $\eta$ is decreased from 1 to 0.3 for the same $r_{T C I}$ value (see Fig. 7.4d). This is due to the fact that a larger area of the ceramics is considered per TE leg when $\eta$ is reduced, hence larger heat losses are produced towards the heat sinks. Secondly, when $\eta$ is kept fixed and $r_{T C l}$ changes from 0 to $10^{-5} \mathrm{~m}^{2} \mathrm{KW}^{-1}$, an increase of the semicircle is observed (see Fig. 7.4d), although this change is less significant than the previous variation caused by the change in $\eta$ for the $r_{T C l}$ values considered. This increase in the semicircle occurs since the thermal contact resistance reduces the heat flow towards the ceramics and hence the heat losses, allowing the temperature difference between both sides of the TE legs to be somewhat larger. These variations in the contacted cases can be quantified by means of the dc resistance, which is the steady-state impedance function $[\mathrm{Z}(\omega \rightarrow 0)]$, and for the equivalent circuit of Fig. 7.5a takes the form,

$$
Z(\omega \rightarrow 0)=R_{\Omega}+\left[R_{T E}^{-1}+\left(R_{T C 1}+R_{S / C}+R_{h 3}+R_{C}\right)^{-1}\right]^{-1}
$$

being $R_{S / C}=4 N S^{2} T_{i} r_{S / C} \eta_{M} / A$, and $r_{S / C}$ the expression for the spreading-constriction resistance [see Eq. (A.9.31)] obtained by Yovanovich et al. [15] for steady state conditions $(\omega \rightarrow 0)$. Eq. (7.37) shows that the dc resistance $[Z(\omega \rightarrow 0)]$ is given by the elements $R_{T C l}, R_{S / C}, R_{h 3}$, and $R_{C}$ in addition 
to the $R_{\Omega}$ and $R_{T E}$ of the ideal model [5]. Since the series connection of the elements $R_{T C l}, R_{S / C}$, $R_{h 3}$, and $R_{C}$ is in parallel with $R_{T E}$, an increase in any of these elements will increase the dc resistance. Consequently, an increase in $r_{T C l}$ increases $R_{T C l}$, and thus the dc resistance, as previously discussed. Moreover, a reduction in $\eta$ increases $R_{S / C}$ and decreases $R_{h 3}$, and $R_{C}$. The latter decrease is more prominent than the $R_{S / C}$ increase and produces a significant reduction of the dc resistance, as previously mentioned.
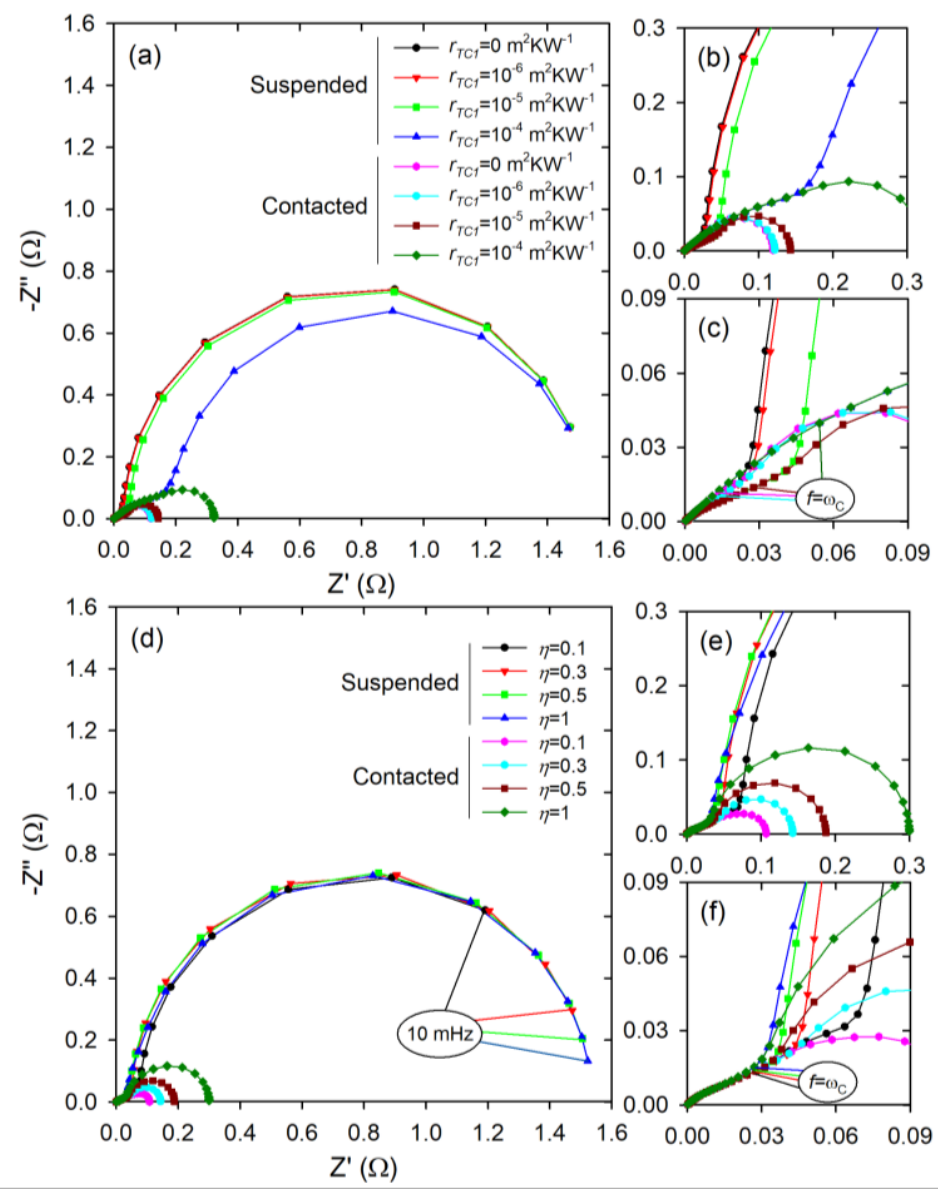

Fig. 7.6. (a, b, c) Impedance spectroscopy simulations when $L_{M}=0$ for different values of $r_{T C l}$ and $\eta=0.3$. (d, e, f) Impedance spectroscopy simulations when $L_{M}=0$ for different values of $\eta$ and $r_{T C l}=10^{-5} \mathrm{~m}^{2} \mathrm{KW}^{-1}$. The plots in (b), (c), (e), and (f) are magnifications of the bottom left part (same axis units). The four larger spectra simulations in each plot consider a module suspended in vacuum, and the other four consider it in contact with ideal heat sinks with a thermal contact resistivity $r_{T C}=1 / h_{3}=10^{-4} \mathrm{~m}^{2} \mathrm{KW}^{-1}$.

In order to evaluate in more detail the effect of $r_{T C l}$ in standard modules, simulations were performed varying $r_{T C l}$ while keeping a value of $\eta=0.3$, as shown in Fig. 7.6a. It can be observed for the case of modules suspended that all the spectra tend to the same value when $f \rightarrow 0$ (right part 
of the spectra), since $\eta$ remains constant (see Fig. 7.6a). However, at higher frequencies, significant differences exist. As mentioned before, $r_{T C l}$ produces an increase of the linear part, which can be quite significant for high $r_{T C l}$ values, and even produce slight variations of the slope in different regions as observed in the magnifications of Fig. 7.6b and Fig. 7.6c. Focusing on the impedance spectra of the contacted TE modules, their semicircle increases with $r_{T C l}$, since more heat flow towards the ceramics is blocked, as previously mentioned, and the temperature difference between the sides of the TE elements becomes higher. The effect of $r_{T C l}$ in the dc resistance can be quantified using Eq. (7.37), as previously mentioned.On the other hand, to evaluate in more detail the effect of the filling factor, simulations of different values of $\eta$ for a fixed value of $r_{T C l}=10^{-5} \mathrm{~m}^{2} \mathrm{KW}^{-1}$ are shown in Fig. 7.6d. It is observed that an increase in $\eta$ produces the delay previously mentioned (see the values of the $10 \mathrm{mHz}$ frequency and $f=\omega_{C}$ in Fig. 7.6d and Fig. 7.6f, respectively).On the other hand, Fig. 7.6e shows a clear reduction of the semicircle for lower values of $\eta$ when the TE module is contacted, which is again attributed to the higher area exposed to heat removal. To complete this analysis, it is worth mentioning that the $\eta$ influence always appear in conventional modules, due to their common architecture, while $r_{T C l}$ might not be present if good junctions are fabricated.

\subsubsection{Effect of the presence of metallic strips considering no metallic strips/ceramics thermal contact resistance}

Fig. 7.7 shows IS simulations when metallic strips are thermally considered (addition of $Z_{W a, M}$ ). Again, both suspended and contacted modules are analyzed, showing both the same behavior at the high frequency part, as previously remarked. Fig. 7.7a (and its magnifications Fig. 7.7b to Fig. 7.7e) show the influence of the metallic strips with no thermal contact resistances existing internally in the TE module $\left(r_{T C l}=r_{T C 2}=0\right)$ for a fixed value of $\eta=0.3$. This configuration corresponds to the equivalent circuits of Fig. 7.8. Under these considerations, when the Peltier heat is injected at the junctions, it diffuses first towards the metallic material, producing a $45^{\circ}$ slope at the highest frequencies that can be observed specially in the spectra with the larger value of $L_{M}$ in Fig. 7.7e. This feature, due to the presence of $Z_{W a, M}$, is difficult to be observed clearly due to the typically high thermal conductivity values of metals, which lead to high and low values of $\omega_{M}$ and $R_{M}$, respectively. After this feature, it is observed that the $45^{\circ}$ straight line rises (vertically) leading to higher absolute values of the slope, being this rise more significant for 
larger $L_{M}$ values, and no observing it for the cases where $L_{M}=0$ (see Fig. 7.7c). This is an important characteristic to identify if the presence of the metallic contact have an impact on the impedance response, and it is due to the accumulation of heat in the metallic material (also introduced by the presence of $Z_{W a, M}$, which occurs simultaneously to the diffusion of heat in the ceramic. In addition to the mentioned characteristics, the presence of the metallic layer also delays the response of the system, as it occurs for the spreading-constriction, and it can be observed looking at the position of $f=10 \mathrm{mHz}$ and $f=\omega_{C}$ in Fig. 7.7a and Fig. 7.7c, respectively.

For the cases where the TE module is contacted, the presence of the metallic strips $\left(Z_{W a, M}\right)$ introduce a temperature drop, like the thermal contact resistances, that is more significant for higher values of $L_{M}$ and slightly increases the semicircle (see Fig. 7.7d). This feature can be quantified by the dc resistance, which is given for this case (equivalent circuit of Fig. 7.8a) by,

$$
Z(\omega \rightarrow 0)=R_{\Omega}+\left[R_{T E}^{-1}+\left(R_{S / C}+R_{h 3}+R_{C}+R_{M}\right)^{-1}\right]^{-1}
$$

where now $R_{M}$ is present due to the metallic strips. Since an increase in $L_{M}$ leads to an increase of $R_{M}$ [see Eq. (7.22)], it also produces an increase in the dc resistance.

Fig. 7.7f (and its magnifications, Fig. 7.7g to Fig. 7.7j) show the same case as before, considering the influence of the metallic strips with $r_{T C 2}=0 \mathrm{~m}^{2} \mathrm{KW}^{-1}, \eta=0.3$, but now introducing $r_{T C l}=10^{-5} \mathrm{~m}^{2} \mathrm{KW}^{-1}$. This case corresponds to the equivalent circuits of Fig. 7.9. When under these considerations $L_{M}$ is varied, differences appear due to the introduction of $r_{T C l}$, since it reduces the heat transfer towards the metallic strip and hence the heat accumulation in it, causing at the highest frequencies a shift of the impedance signal towards the real axis, and even showing a horizontallike part for thick metallic strips (see Fig. 7.7j). Regarding the contacted modules, it should be noted that, as occurred in Fig. 7.7d, a slight increase of the semicircles with $L_{M}$ is also happening in Fig. 7.7i. However, it can be observed that the semicircles in Fig. 7.7i are somewhat larger (impedance values at the lowest frequencies $\approx 0.15 \Omega$ ) than those in Fig. $7.7 \mathrm{~d}$ (impedance values at the lowest frequencies $\approx 0.12 \Omega$ ), due to the presence of $r_{T C l}$, which also affects the dc resistance, given by,

$$
Z(\omega \rightarrow 0)=R_{\Omega}+\left[R_{T E}^{-1}+\left(R_{T C 1}+R_{S / C}+R_{h 3}+R_{C}+R_{M}\right)^{-1}\right]^{-1} .
$$


Chapter 7
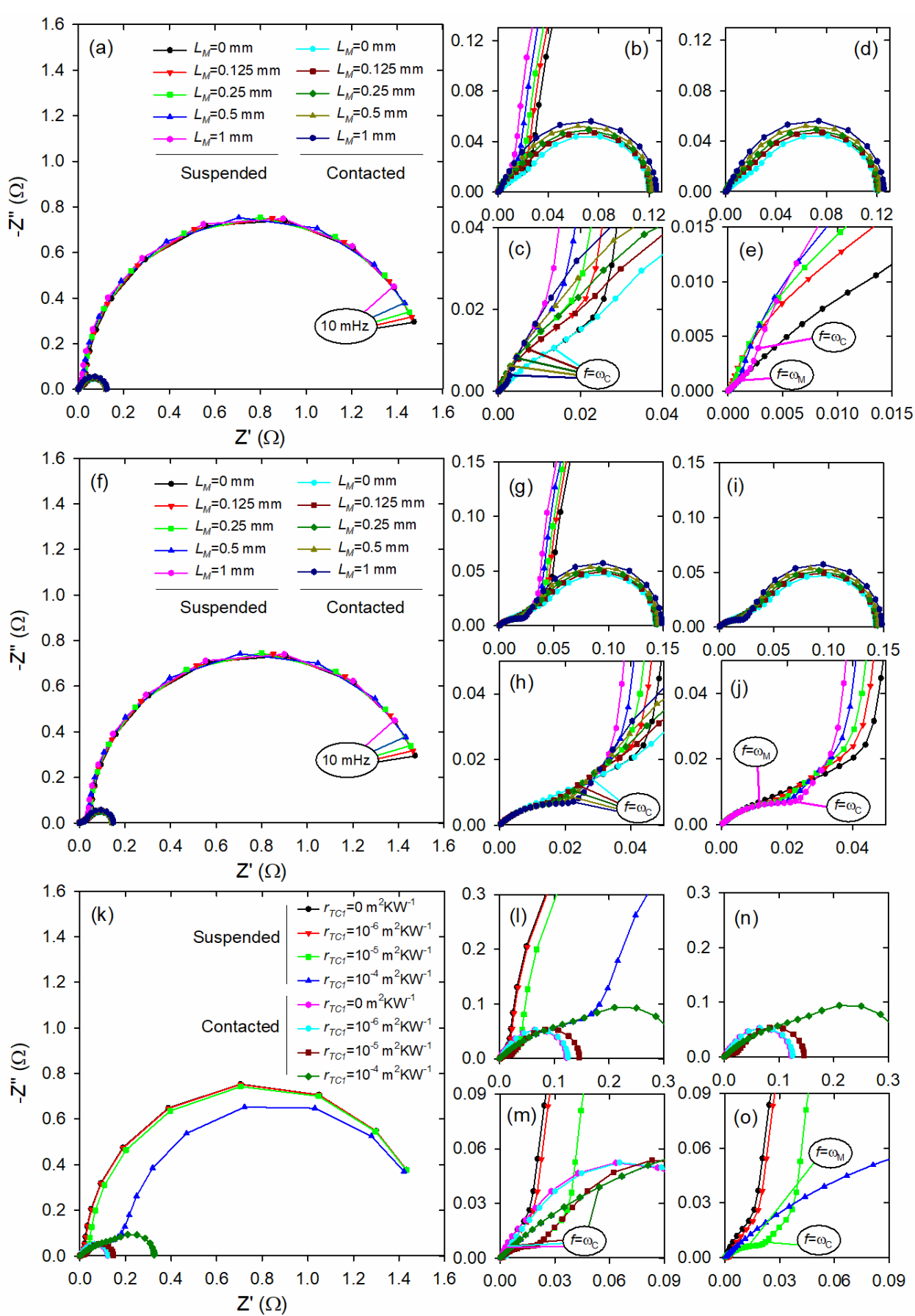

Fig. 7.7. Impedance spectroscopy simulations for different values of $L_{M}$ for (a-e) $r_{T C I}=0 \mathrm{~m}^{2} \mathrm{KW}^{-1}$ and (f-j) for $r_{T C l}=10^{-5} \mathrm{~m}^{2} \mathrm{KW}^{-1}$. (k-o) Simulations for different values of $r_{T C l}$ considering a fixed value of $L_{M}=0.5$ $\mathrm{mm}$. In all cases $\eta=0.3$, and $r_{T C 2}=0 \mathrm{~m}^{2} \mathrm{KW}^{-1}$. The plots (b-e), (g-j) and (l-o) are magnifications of the bottom left part (same axis units) of plots (a), (f), and (k), respectively. The four larger spectra simulations of each graph consider the module suspended in vacuum, and the other four consider it in contact with a heat source/sink with a thermal contact resistivity of $10^{-4} \mathrm{~m}^{2} \mathrm{KW}^{-1}\left(r_{T C}=1 / h_{3}=10^{-4} \mathrm{~m}^{2} \mathrm{KW}^{-1}\right)$. The four larger 
spectra simulations in each plot consider a module suspended in vacuum, and the other four consider it in contact with ideal heat sinks with a thermal contact resistivity $r_{T C}=1 / h_{3}=10^{-4} \mathrm{~m}^{2} \mathrm{KW}^{-1}$.

(a)

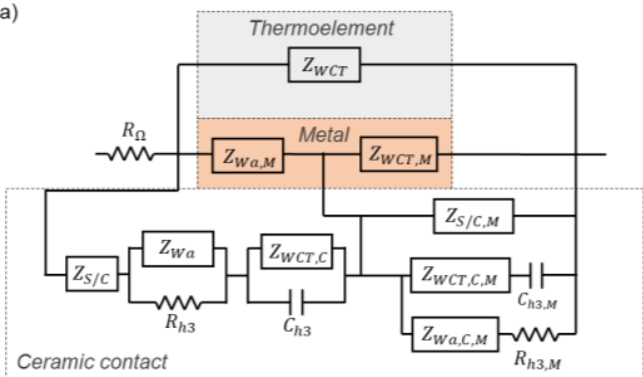

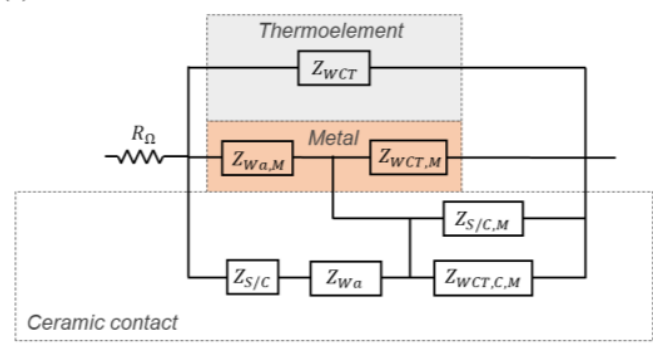

Fig. 7.8. Simplified equivalent circuits of thermoelectric modules when $h_{2}=0, r_{T C l}=0$, and $r_{T C 2}=0$ for (a) contacted modules and (b) suspended modules in vacuum $\left(h_{3}=0\right)$.

(a)

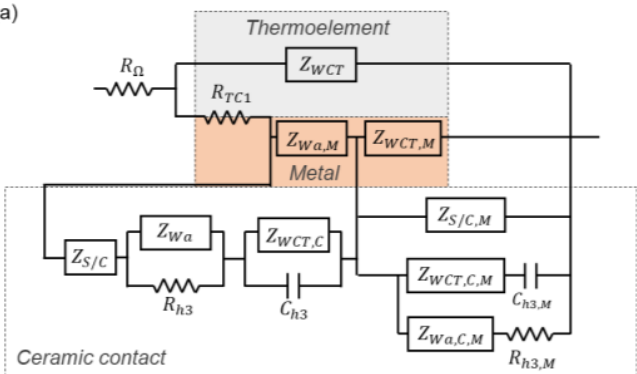

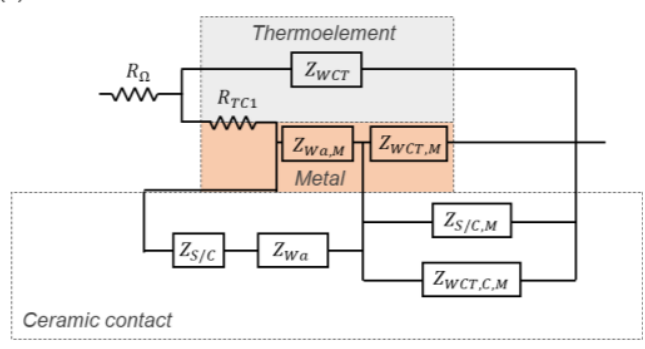

Fig. 7.9. Simplified equivalent circuits of thermoelectric modules when $h_{2}=0$, and $r_{T C 2}=0$ for (a) contacted modules and (b) suspended modules in vacuum $\left(h_{3}=0\right)$.

Finally, Fig. 7.7k (and its magnifications, Fig. 7.71 to Fig. 7.7o) evaluates how the impedance is modified for different values of $r_{T C l}$ for a fixed length of the metallic strip ( $L_{M}=0.5 \mathrm{~mm}$ ), and keeping $r_{T C 2}=0$ and $\eta=0.3$. In this case, the frequencies are not shifted towards higher frequency values, and all the suspended modules spectra tends to terminate at the same point (see Fig. 7.7k). Fig. 7.7o shows that significant changes may occur at high frequencies when the thermal contact resistance is increased. In general, the high frequency part becomes more prominent when the contact worsens, leading to different features, which can even produce horizontal-like trends (see the case for $r_{T C l}=10^{-5} \mathrm{~m}^{2} \mathrm{KW}^{-1}$ in Fig. 7.7o). Fig. 7.7d and Fig. 7.7e show the slower semicircle and the increase of it for the module sandwiched than the previous case, where $r_{T C l}=0 \mathrm{~m}^{2} \mathrm{KW}^{-1}$. regarding contacted modules, the effect of increasing $r_{T C l}$ leads to the increase of the semicircle (see Fig. 7.7k and Fig. 7.7n), more than the increase of the metal thickness of Fig. 7.7i, since $r_{T C l}$ produces a higher temperature drop than a thin layer of metal. 
We would like to also mention that it should be noticed that although the simulations in Fig. 7.7j and Fig. 7.7o considers the presence of the metallic strips, none of them show lines with slopes above $45^{\circ}$ at the highest frequency part, as it was the case in Fig. 7.7e. Thus, the fact of not observing a slope above $45^{\circ}$ in the high frequency part does not discard that the metallic strip has no influence.

\subsubsection{Effect of the presence of metallic strips considering the metallic strips/ceramics thermal contact resistance}

Simulations of Fig. 7.10 evaluate the case when metallic strips are present and a thermal contact resistance between the metallic material and the ceramics $\left(r_{T C 2}\right)$ exists. In all the simulations the thermal contact resistance between the thermoelements and the metallic strips is neglected $\left(r_{T C l}=0 \mathrm{~m}^{2} \mathrm{KW}^{-1}\right)$, thus, the corresponding equivalent circuits are those of Fig. 7.11.

Fig. 7.10a (and its magnifications Fig. 7.10b and Fig. 7.10c) show simulations for different values of $r_{T C 2}$ considering $\eta=0.3$, and $L_{M}=0.5 \mathrm{~mm}$. At high frequencies, it can be observed that an increase in $r_{T C 2}$ shifts the onset of the large semicircle towards both higher absolutes values of the imaginary and real impedances, leading to a large shoulder for the highest $r_{T C 2}$ value (see Fig. $7.10 \mathrm{~b})$. This is due to the enhancement of the accumulation of heat in the metal due to the lower heat flow towards the ceramics, caused by the presence of $r_{T C 2}$. At low frequencies, it should be noticed for suspended modules that in all cases the steady state condition (when $f \rightarrow 0$ ) is reached simultaneously, thus, delays are not produced by $r_{T C 2}$, as it was also the case for $r_{T C l}$ (Fig. 7.7g). For contacted modules, an increase of the semicircle at low frequencies is observed when $r_{T C 2}$ increases (see Fig. 7.10a and Fig. 7.10b), as it was also the case for $r_{T C I}$. This can be predicted by the dc resistance of the equivalent circuit of Fig. 7.11a, that now includes $R_{T C 2}$,

$$
Z(\omega \rightarrow 0)=R_{\Omega}+\left[R_{T E}^{-1}+\left(R_{T C 2}+R_{S / C}+R_{h 3}+R_{C}+R_{M}\right)^{-1}\right]^{-1} .
$$

Fig. 7.10d (and its magnifications Fig. 7.10e and Fig. 7.10f) show impedance simulations for different values of $\eta$ when $L_{M}=0.5 \mathrm{~mm}$, and $r_{T C 2}=10^{-5} \mathrm{~m}^{2} \mathrm{KW}^{-1}$. The decrease of $\eta$ favors the appearance of a shoulder at high frequencies (Fig. 7.10e), which is a similar feature to that caused by the increase of $r_{T C 2}$ (Fig. 7.10b). However, contrary to the $r_{T C 2}$ case that does not modify the dynamics of the system, the decrease of $\eta$ delays the process to reach the steady state for suspended modules (see the frequency point of $10 \mathrm{mHz}$ in Fig. 7.10d). For the case of contacted 


\section{Chapter 7}

modules, a decrease in $\eta$ reduces the semicircle, due to the presence of a larger ceramic area per area of thermoelement, as discussed above and evident from the dc resistance of Eq. (7.40).
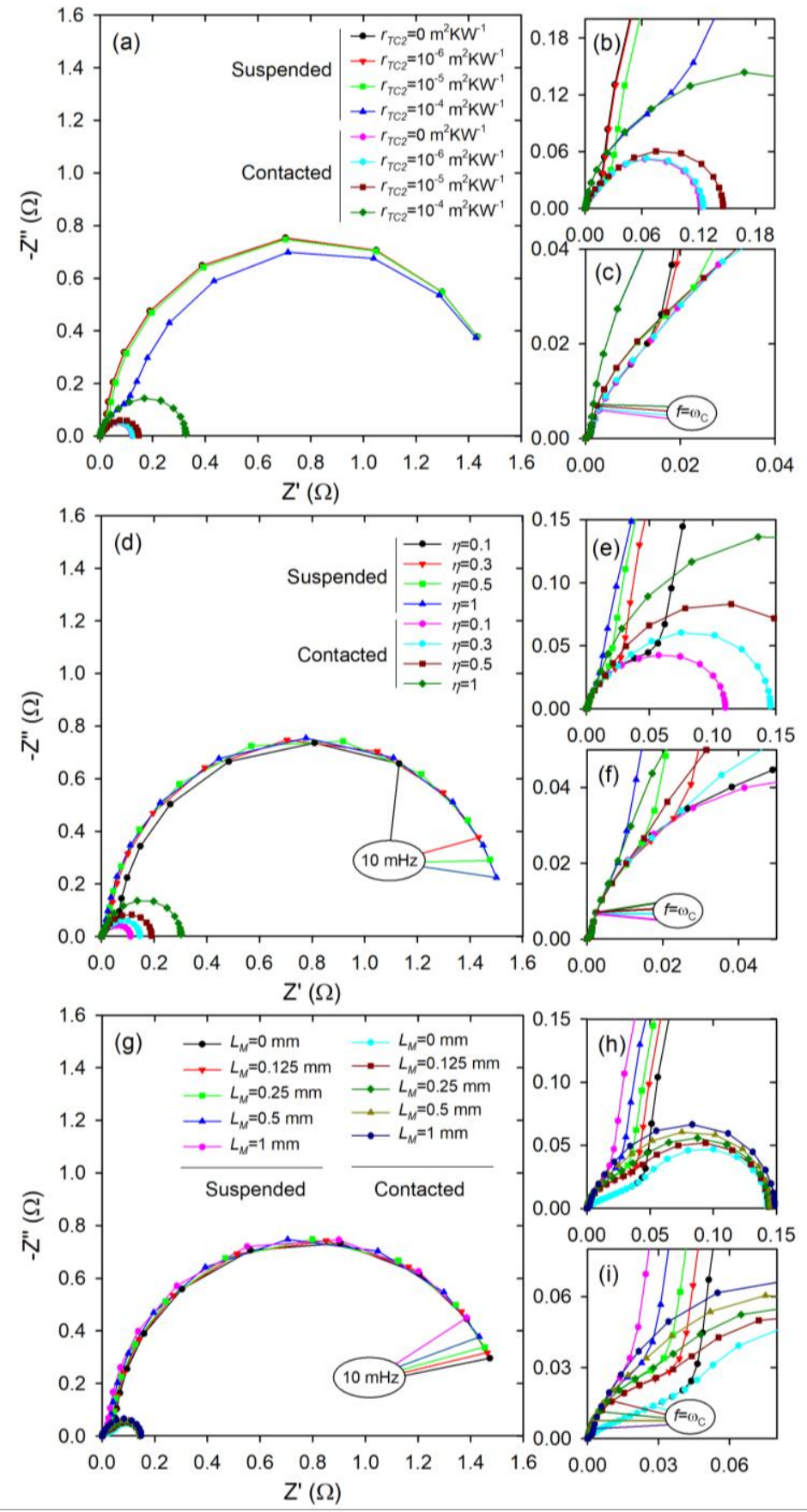

Fig. 7.10. (a-c) Impedance spectroscopy simulations for different values of $r_{T C 2}, \eta=0.3$, and $L_{M}=0.5 \mathrm{~mm}$. (d-f) Impedance simulations for different values of $\eta, L_{M}=0.5 \mathrm{~mm}$, and $r_{T C 2}=10^{-5} \mathrm{~m}^{2} \mathrm{KW}^{-1}$. (g-i) Impedance 
simulations for different values of $L_{M}, \eta=0.3$, and $r_{T C 2}=10^{-5} \mathrm{~m}^{2} \mathrm{KW}^{-1}$. For all cases $r_{T C l}=0 \mathrm{~m}^{2} \mathrm{KW}^{-1}$. Plots $(\mathrm{b}$, c), (e, f), and (h, i) are magnifications of the bottom left part (same axis units) of plots (a), (d), and (g), respectively. The four larger spectra simulations in each plot consider a module suspended in vacuum, and the other four consider it in contact with ideal heat sinks with a thermal contact resistivity $r_{T C}=1 / h_{3}=10^{-4}$ $\mathrm{m}^{2} \mathrm{KW}^{-1}$.
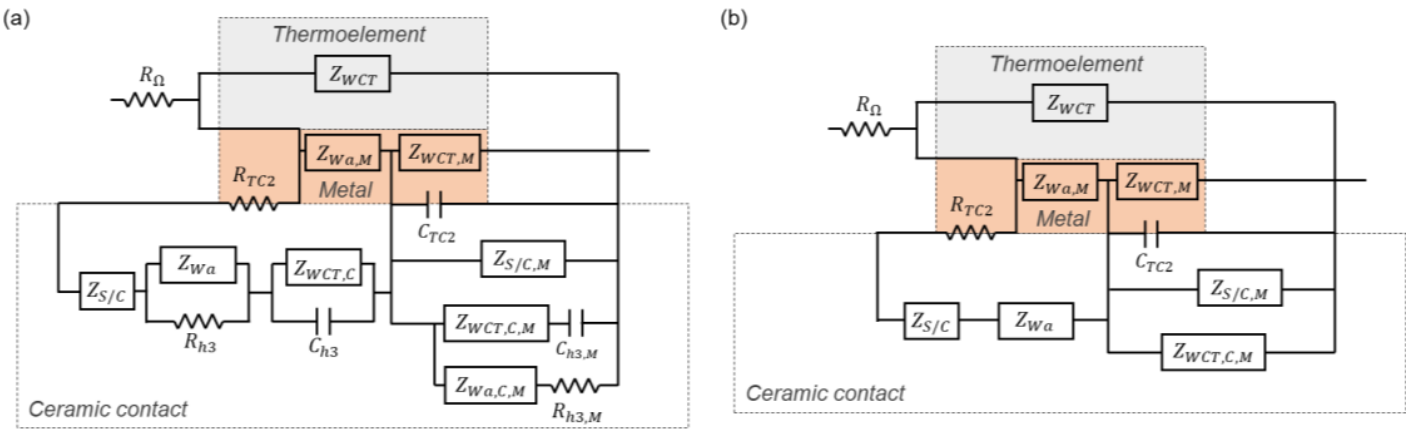

Fig. 7.11. Simplified equivalent circuits of thermoelectric modules when $h_{2}=0$, and $r_{T C l}=0$ for (a) contacted modules and (b) suspended modules in vacuum $\left(h_{3}=0\right)$.

Fig. 7.10g (and its magnifications Fig. 7.10h and Fig. 7.10i) show simulations at different values of $L_{M}$ considering $\eta=0.3$, and $r_{T C 2}=10^{-5} \mathrm{~m}^{2} \mathrm{KW}^{-1}$. It can be observed at high frequencies that even a small thickness of the metallic material produces a significant variation with respect to the case where $L_{M}=0$ (see Fig. 7.10i). It is also shown, in the same figure, that an increase in $L_{M}$ significantly rises (vertically) the impedance response, which is due to the more prominent absorption of heat in the metallic element when its thickness is increased. As it was observed in Fig. 7.7f, the increase in the thickness of the metal also slows down the dynamics of the system for suspended modules, as shown by $f=10 \mathrm{mHz}$ and $f=\omega_{C}$ in Fig. $7.10 \mathrm{~g}$ and Fig. 7.10i, respectively. Moreover, for contacted modules, a slight increase of the semicircle is observed when $L_{M}$ increases, similarly to Fig. 7.7i.

\subsubsection{All elements and effects considered}

Fig. 7.13 shows the simulations of the whole equivalent circuit of Fig. 7.3, which combines all the effects previously analyzed for a TE module with a $\eta=0.3$. Fig. 7.13a (and its magnifications Fig. 7.13b and Fig. 7.13c) show the influence of $r_{T C l}$ considering $L_{M}=0.5 \mathrm{~mm}$, and $r_{T C 2}=10^{-5} \mathrm{~m}^{2} \mathrm{KW}^{-1}$. It can be observed that a similar result to that found in Fig. 7.7(g-i) for the case when $r_{T C 2}$ was neglected is obtained. 


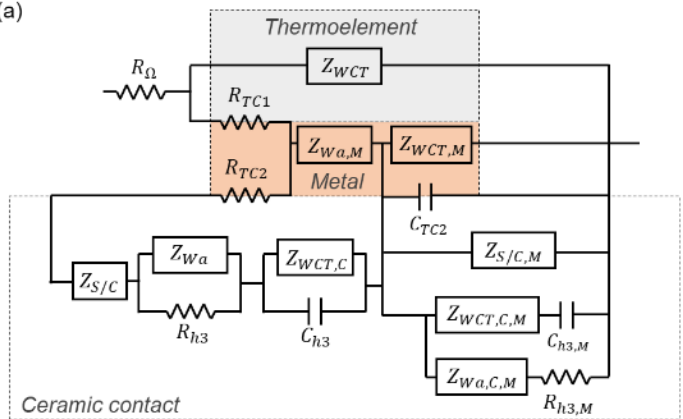

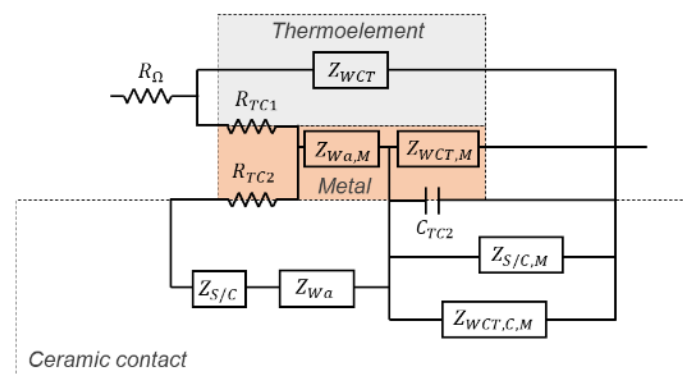

Fig. 7.12. Simplified equivalent circuits of thermoelectric modules when $h_{2}=0$ for (a) contacted modules and (b) suspended modules in vacuum $\left(h_{3}=0\right)$.

Fig. 7.13d (and its magnifications Fig. 7.13e and Fig. 7.13f) show the influence of $L_{M}$ for $r_{T C l}=10^{-5} \mathrm{~m}^{2} \mathrm{KW}^{-1}$, and $r_{T C 2}=10^{-5} \mathrm{~m}^{2} \mathrm{KW}^{-1}$. This case is again very similar to the case in Fig. $7.7(\mathrm{~d}-$ f) where $r_{T C 2}$ was neglected. The presence of $r_{T C 2}$ only makes slightly more curved the zone with the most significant variations in Fig. 7.13f with respect to Fig. 7.7j, where this gentle curvature is not observed. Finally, Fig. 7.13g (and its magnifications Fig. 7.13h and Fig. 7.13i) show impedance simulations for different values of $r_{T C 2}$ considering $L_{M}=0.5 \mathrm{~mm}$, and $r_{T C l}=10^{-5} \mathrm{~m}^{2} \mathrm{KW}^{-}$ ${ }^{1}$. It can be observed in the high frequency part (Fig. 7.13i) that all the simulations are identical until $Z ` 0.02 \Omega$, since the heat flow have not yet arrived at the metallic strip/ceramic interface. At higher Z' values the trend observed is very similar to that found in Fig. 7.10(a-c), where $r_{T C 1}$ was not considered, thus, the inclusion of $r_{T C l}$ have a more significant influence in the impedance response than $r_{T C 2}$. Regarding the dc resistance for contacted modules (equivalent circuit of Fig. 7.12a), when the complete equivalent circuit is considered it takes the form,

$$
Z(\omega \rightarrow 0)=R_{\Omega}+\left[R_{T E}^{-1}+\left(R_{T C 1}+R_{T C 2}+R_{S / C}+R_{h 3}+R_{C}+R_{M}\right)^{-1}\right]^{-1}
$$

We would like to remark that it should be noticed that the analysis performed in this whole section is for the most typical commercial modules, and deviations may occur if different values of the parameters are adopted, or the architecture of the devices significantly vary. 
Chapter 7
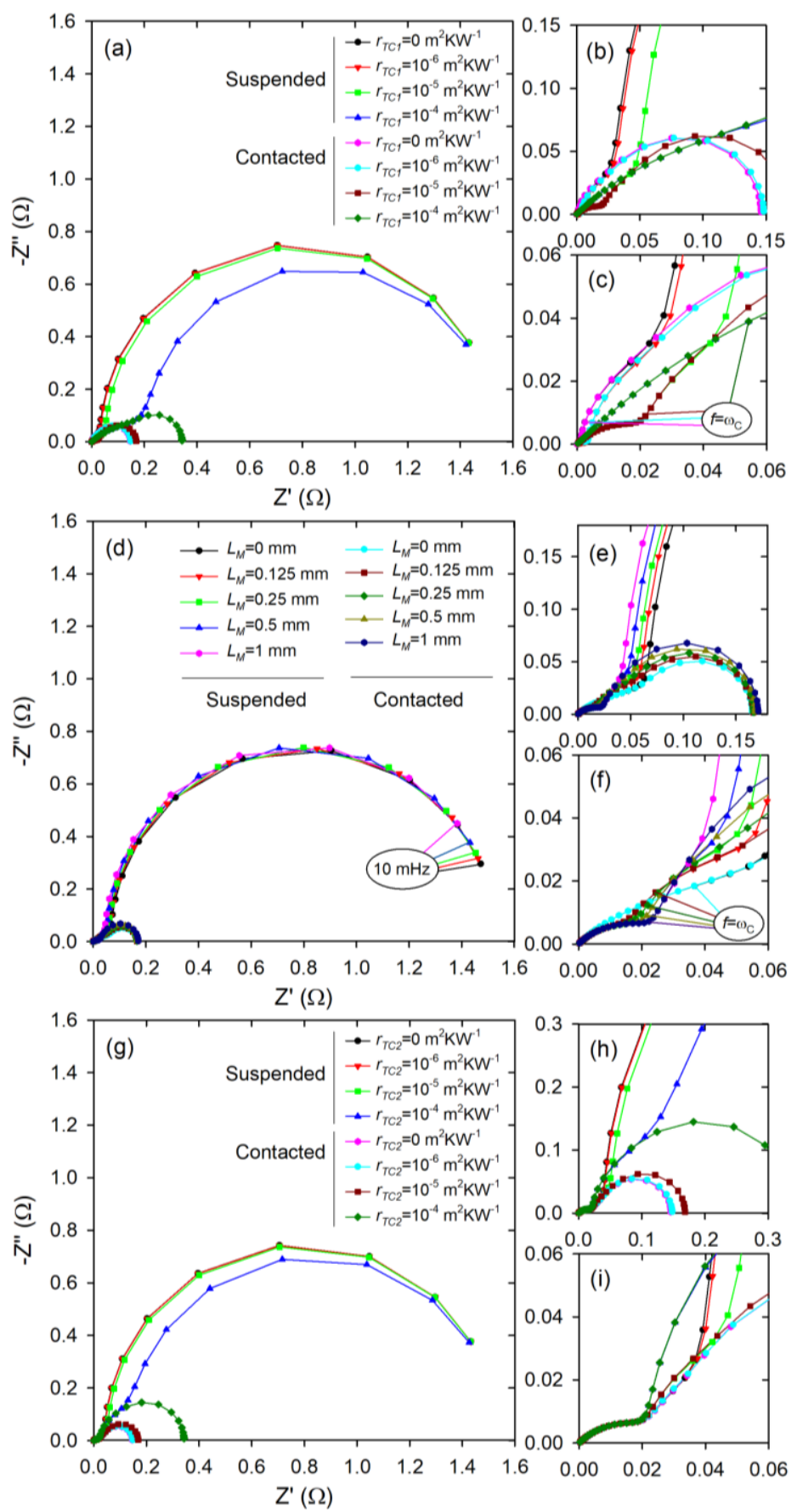

Fig. 7.13. (a-c) Impedance spectroscopy simulations for different values of $r_{T C 1}, L_{M}=0.5 \mathrm{~mm}$, and $r_{T C 2}=10^{-}$ ${ }^{5} \mathrm{~m}^{2} \mathrm{KW}^{-1}$. (d-f) Impedance simulations for different values of $L_{M}, r_{T C l}=10^{-5} \mathrm{~m}^{2} \mathrm{KW}^{-1}$, and $r_{T C 2}=10^{-5} \mathrm{~m}^{2} \mathrm{KW}^{-}$ ${ }^{1}$. (g-i) Impedance simulations for different values of $r_{T C 2}, L_{M}=0.5 \mathrm{~mm}$, and $r_{T C l}=10^{-5} \mathrm{~m}^{2} \mathrm{KW}^{-1}$. For all cases $\eta=0.3$. Plots (b, c), (e, f), and (h, i) are magnifications of the bottom left part (same axis units) of plots (a), (d), and (g), respectively. The four larger spectra simulations in each plot consider a module suspended in 
vacuum, and the other four consider it in contact with ideal heat sinks with a thermal contact resistivity $r_{T C}=1 / h_{3}=10^{-4} \mathrm{~m}^{2} \mathrm{KW}^{-1}$.

\subsection{Experimental results}

In order to evaluate the capabilities of the new comprehensive equivalent circuit developed, the impedance response of three commercial Bi-Te TE modules from different manufacturers were measured: Module 1 (Custom Thermoelectric, ref. 04801-933B-34RB), Module 2 (Interm, ref. CBM-88), and Module 3 (European Thermodynamics, ref. 693-7080). The dimensions, $N, L$, $L_{M}, L_{C}, A, \eta_{M}$, and $\eta$ of the three modules and the current amplitude $I_{a c}$, the number of points (logarithmically distributed in the frequency range), and the frequency range of the IS measurements performed to each module, can be found in Table 7.1. The current amplitude and the frequency range were optimized for each TE module as described in ref. [18]. Amplitude optimization basically consists in identifying the lowest possible amplitude with no noise in the spectra. The frequency optimization was performed to ensure a proper number of points in the regions of interest in the spectra, mainly in the high frequency part. All the measurements were performed with the modules suspended under vacuum $\left(<10^{-3}\right.$ mbar $)$ with a PGSTAT30 potentiostat (Metrohm Autolab B. V.) equipped with a FRA2 impedance module and a BOOSTER10A, which is capable to amplify the maximum current of the equipment to $10 \mathrm{~A}$. The current booster is not needed for the current amplitudes used in the measurements, however, it reduced the measurement noise [17].

Table 7.1. Specifications of the commercial TE modules used in this study and experimental impedance spectroscopy parameters employed.

\begin{tabular}{|c|c|c|c|c|c|c|c|c|c|c|c|}
\hline Name & $\begin{array}{c}\text { Size } \\
\left(\mathbf{m m}^{2}\right)\end{array}$ & $N$ & $\begin{array}{c}L \\
(\mathbf{m m})\end{array}$ & $\begin{array}{c}L_{M} \\
(\mathbf{m m})\end{array}$ & $\begin{array}{c}L_{C} \\
(\mathbf{m m})\end{array}$ & $A\left(\mathbf{m m}^{2}\right)$ & $\eta_{M}$ & $\eta$ & $\begin{array}{c}I_{a c} \\
(\mathbf{m A})\end{array}$ & Points & $\begin{array}{l}\text { Freq. } \\
\text { range }\end{array}$ \\
\hline Module 1 & $10 \times 10$ & 48 & 0.55 & 0.03 & 0.525 & $0.6 \times 0.6$ & 0.71 & 0.35 & 60 & 40 & $\begin{array}{c}50 \mathrm{mHz}- \\
10 \mathrm{kHz}\end{array}$ \\
\hline Module 2 & $14 \times 14$ & 39 & 1.1 & 0.06 & 0.5 & $0.6 \times 0.6$ & 0.50 & 0.14 & 60 & 50 & $\begin{array}{c}10 \mathrm{mHz} \text { - } \\
10 \mathrm{kHz}\end{array}$ \\
\hline Module 3 & $40 \times 40$ & 127 & 1.2 & 0.3 & 0.75 & $1.3 \times 1.3$ & 0.67 & 0.27 & 50 & 50 & $\begin{array}{c}20 \mathrm{mHz}- \\
20 \mathrm{kHz}\end{array}$ \\
\hline
\end{tabular}

Fig. 7.14a, Fig. 7.15a, and Fig. 7.16a show the experimental IS measurements performed to the three TE modules of Table 7.1. It can be observed that they all differ significantly in their response at high frequency (inset of each figure). Typically, a fitting to the experimental results 
using a suitable equivalent circuit is performed to obtain the relevant information of the system. However, due to the large number of elements present in the comprehensive equivalent circuit of Fig. 7.3, fittings did not converge when they were tried. On the other hand, a qualitative analysis can be performed using a systematic procedure consisting in different simulations adapted to each of the modules.

For the purpose of this qualitative analysis, we have developed a Matlab code (provided in Annex 9.2) which simulates the module under analysis [once all its dimensions (data in Table 7.1) are entered and using estimated values for the material's properties] assuming no thermal contact resistances and systematically showing the evolution of the spectra for different values of the two possible thermal contact resistivities $\left(r_{T C l}\right.$ and $\left.r_{T C 2}\right)$. By comparison of the experimentally measured spectrum with the simulations provided by the code, it can be estimated if $r_{T C l}$ and $r_{T C 2}$ are present, and their approximate values.

We have applied this analysis to the modules in Table 7.1. The simulation results are shown for Module 1, Module 2, and Module 3 in Fig. 7.14(b-e), Fig. 7.15(b-e), and Fig. 7.16(b-e), respectively. It should be noticed that in all the simulations the ohmic resistance $R_{\Omega}$ has been omitted for clarity. For all the modules, it can be observed in the (b) plots of the figures that the diameters of the simulated semicircles are similar to the ones obtained experimentally [(a) plots of the figures], which indicates that the estimated materials' properties are reasonable. In the (c) plots of the figures (black circles) it is shown the high frequency magnification of the simulations considering ideal thermal contacts $\left(r_{T C l}=r_{T C 2}=0\right)$. Comparing these simulations with the experimental measurements [insets of the (a) plots of the figures], it can be observed that significant differences exist between them, which points to the existence of $r_{T C l}$ and/or $r_{T C 2}$ in the modules. The other simulations in the (c) plots of the figures (green squares), are the ones with the best match to the experimental spectrum after analyzing the results from the Matlab code. In all cases the best match corresponds to simulations with $r_{T C 2}=0$, being the metallic strip/TE element interface $\left(r_{T C l}\right)$ the one responsible for the deviations found with respect to the simulations with ideal thermal junctions (black circles). This is not a surprising result, since typically the most problematic junction in TE devices is the one formed by the metallic strips and the thermoelements [19], and the thermal contact between the metallic strips and the ceramic layer is more robust and less problematic. 
In the (d) plots of Fig. 7.14, Fig. 7.15, and Fig. 7.16, the simulations obtained from the Matlab code by considering $r_{T C 2}=0$ and systematically varying $r_{T C l}$ are shown for Modules 1, 2 and 3, respectively. In addition, the (e) plots of the same figures show the simulations varying $r_{T C 2}$ while keeping a fixed value for $r_{T C I}$. For Module 1, it can be observed in Fig. $7.14 \mathrm{~d}$ that higher values of $r_{T C l}$ lead to lower slopes and to a more linear trend, which is the experimentally observed characteristic in the inset of Fig. 7.14a. When a fixed value of $r_{T C l}$ is adopted $\left(3 \times 10^{-6} \mathrm{~m}^{2} \mathrm{KW}^{-1}\right)$ and $r_{T C 2}$ is increased, a curvature becomes more prominent in part of the impedance response (see Fig. 7.14e), which deviates from the experimentally observed result (inset of Fig. 7.14a). Hence, the most similar simulations to the experimental results are the ones with $r_{T C l}$ values between $3 \times 10^{-6}$ to $5 \times 10^{-6} \mathrm{~m}^{2} \mathrm{KW}^{-1}$ and no thermal contact resistance between metallic strips and external ceramic layers.
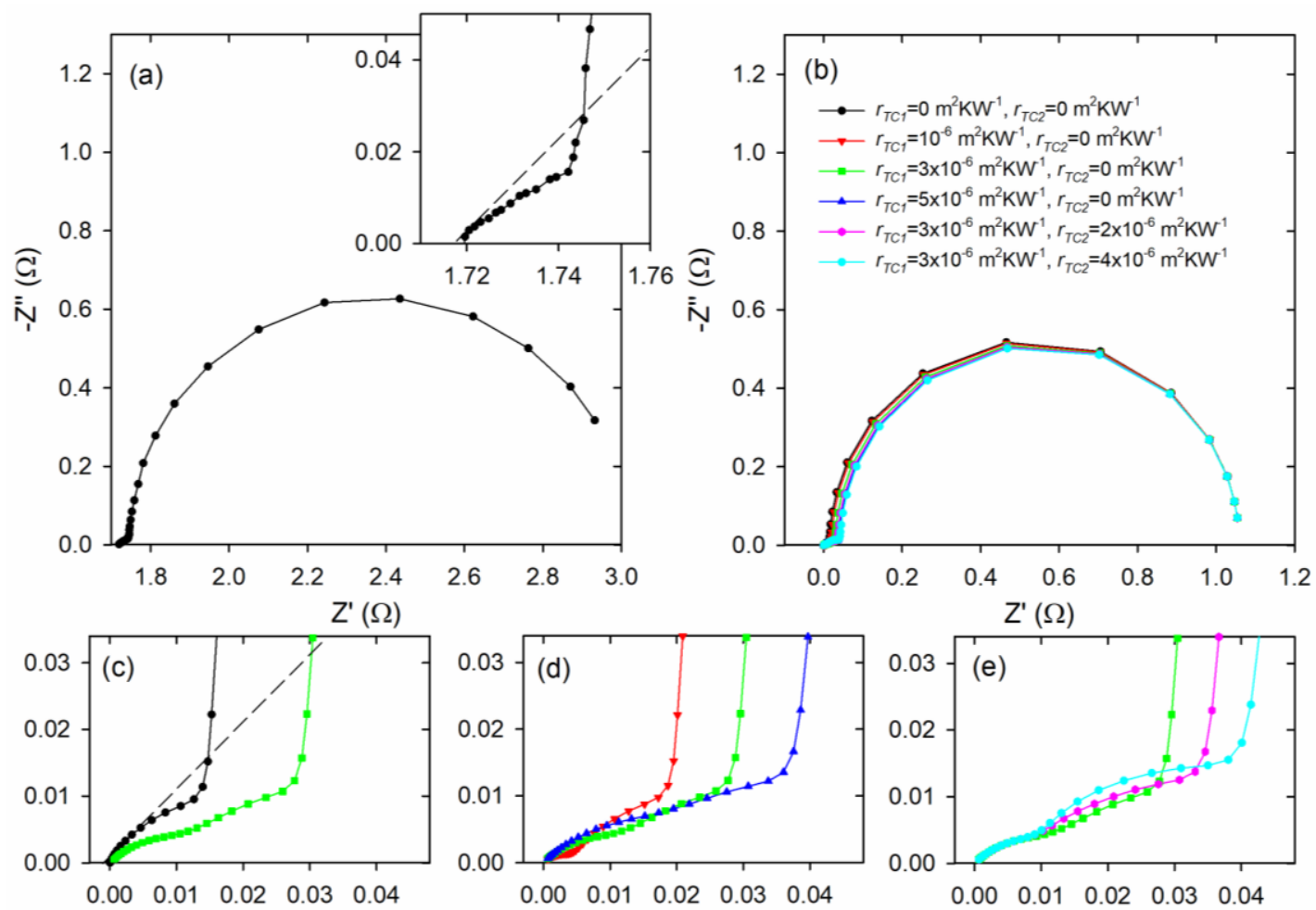

Fig. 7.14. (a) Experimental impedance spectroscopy measurement of Module 1, including the magnification of the high frequency part in the inset. (b) Impedance simulations performed using the Matlab code in Annex 9.2 for different values of thermal contact resistivities $r_{T C l}$ and $r_{T C 2}$. (c-e) Magnifications of the high frequency part of different spectra in (b). Dashed lines are indications of a $45^{\circ}$ slope. The simulations were made with the geometric parameters of columns 3 to 9 of Table 7.1 and the default material's properties previously used in the equivalent circuit analysis of section 7.3. The ohmic resistance has been omitted in the simulations for clarity. 
For Module 2, it can be observed in Fig. 7.15d that higher values of $r_{T C l}$ result in a wider and more horizontal-like response, which is the experimentally observed feature in the inset of Fig. 7.15a. When a fixed value of $r_{T C l}$ is adopted $\left(7 \times 10^{-6} \mathrm{~m}^{2} \mathrm{KW}^{-1}\right)$ and $r_{T C 2}$ is increased, a curvature becomes again more prominent in part of the impedance response (see Fig. 7.15e), which deviates from the experimentally observed result (inset of Fig. 7.15a). Hence, the most similar simulation to the experimental results is the one with $r_{T C l}=7 \times 10^{-6} \mathrm{~m}^{2} \mathrm{KW}^{-1}$ and no thermal contact resistance between metallic strips and external ceramic layers.
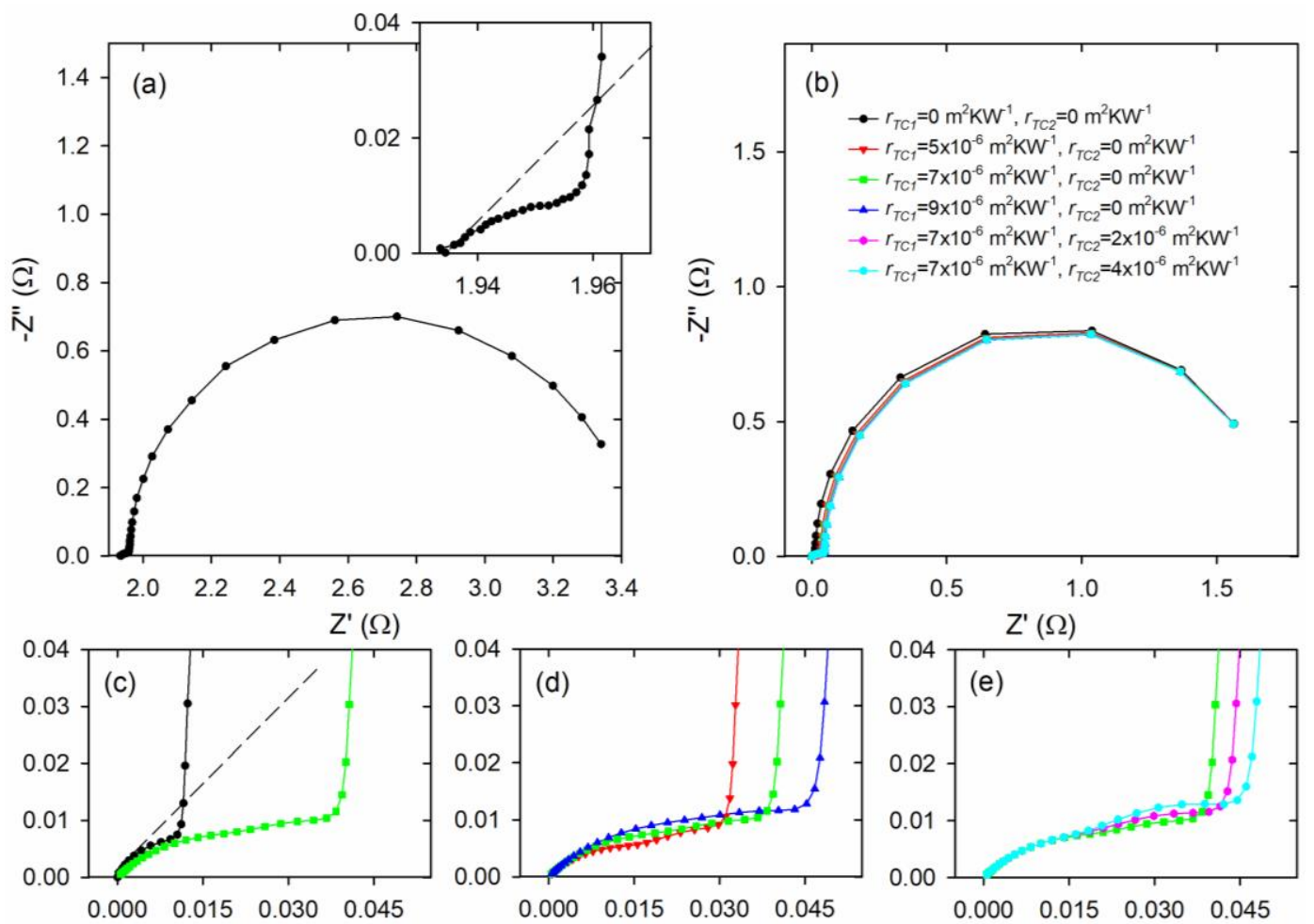

Fig. 7.15. (a) Experimental impedance spectroscopy measurement of Module 2, including the magnification of the high frequency part in the inset. (b) Impedance simulations performed using the Matlab code in Annex 9.2 for different values of thermal contact resistivities $r_{T C l}$ and $r_{T C 2}$. (c-e) Magnifications of the high frequency part of different spectra in (b). Dashed lines are indications of a $45^{\circ}$ slope. The simulations were made with the geometric parameters of columns 3 to 9 of Table 7.1 and the default material's properties previously used in the equivalent circuit analysis of section 7.3. The ohmic resistance has been omitted in the simulations for clarity.

Finally, for Module 3 it can be observed from Fig. 7.16c and d that the addition of $r_{T C l}$ produces the initial curvature followed by the $45^{\circ}$ slope straight line result which is experimentally observed in the inset of Fig. 7.16a. When a fixed value of $r_{T C l}$ is adopted $\left(5 \times 10^{-6} \mathrm{~m}^{2} \mathrm{KW}^{-1}\right)$ and $r_{T C 2}$ is increased, a more prominent curvature appears again in part of the impedance response (see Fig. 
7.16e), although in this case smaller differences exist with respect to Modules 2 and 3. This curvature deviates from the experimentally observed result (inset of Fig. 7.16a) which exhibit a more linear trend in this part. Hence, from this analysis, the most similar simulation to the experimental result is the one with $r_{T C I}=5 \times 10^{-6} \mathrm{~m}^{2} \mathrm{KW}^{-1}$ and no thermal contact resistance between metallic strips and external ceramic layers.
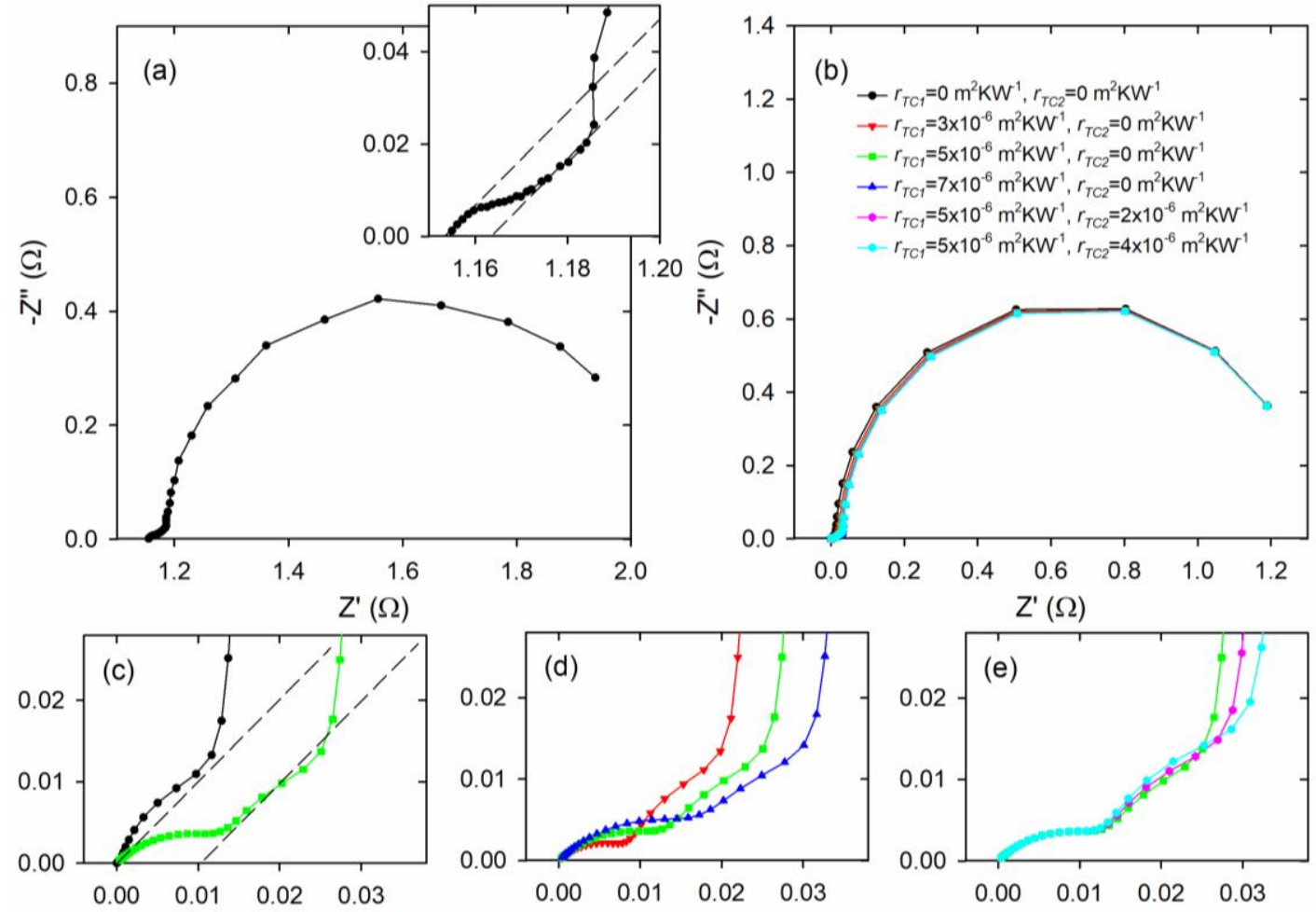

Fig. 7.16. (a) Experimental impedance spectroscopy measurement of Module 1, including the magnification of the high frequency part in the inset. (b) Impedance simulations performed using the Matlab code in Annex 9.2 for different values of thermal contact resistivities $r_{T C l}$ and $r_{T C 2}$. (c-e) Magnifications of the high frequency part of different spectra in (b). Dashed lines are indications of a $45^{\circ}$ slope. The simulations were made with the geometric parameters of columns 3 to 9 of Table 7.1 and the default material's properties previously used in the equivalent circuit analysis of section 7.3. The ohmic resistance has been omitted in the simulations for clarity.

The analysis shown above for the three different commercial modules demonstrate that, although fittings to the experimental results could not be accurately performed, a qualitative analysis considering different combinations of the thermal contact resistivities ( $r_{T C l}$ and $\left.r_{T C 2}\right)$ serves to identify which thermal contact resistance is present and its approximated value. 


\subsection{Conclusions}

A comprehensive equivalent circuit which covers all the key phenomena that affects the performance of TE modules has been developed. The new equivalent circuit includes, as new additions, the thermal influence of the metallic strips, combined with the thermal contact resistance between the metallic strips and the outer ceramic layer. Moreover, a new more accurate spreading-constriction impedance element, which considers the variation of the heat flow in the radial direction at the outer ceramic surfaces, was also developed. Apart from these new additions, the equivalent circuit includes other key different elements previously reported.

Different simulations, that considered a wide number of possible effects, such as the presence of the metallic contacts, thermal contact resistances, and heat losses from different parts of the module, have been performed and analyzed, in order to identify trends that can be present in real devices. It was found that all these effects significantly modify the impedance spectra specially at high frequencies. Once theoretically analyzed, the developed equivalent circuit was used to qualitatively evaluate significant differences that were found in experimental measurements performed to three commercial Bi-Te modules from different suppliers. For this analysis, a Matlab code has been created (see Annex 9.2). The analysis revealed the presence of a thermal contact resistance at the metallic strips/TE elements interface in all the commercial modules, whose value was estimated. No significant influence of the metallic strips/ceramic layers thermal interface was identified. This study opens up the possibility of using IS as a powerful tool to detect thermal contact resistances in TE devices and monitor in great detail possible issues that could appear during their performance or manufacturing process.

\section{Acknowledgments}

The authors acknowledge financial support from the Spanish Agencia Estatal de Investigación under the Ramón y Cajal program (RYC-2013-13970), from the Generalitat Valenciana and the European Social Fund under the ACIF program (ACIF/2018/233) and BEFPI program (BEFPI/2019/030), and from the Universitat Jaume I under the projects UJI-A2016-08 and UJIB2019-50. European Thermodynamics is also acknowledged for supplying thermoelectric devices. 


\section{References}

[1] A. De Marchi, V. Giaretto, S. Caron, A. Tona, A. De Marchi, V. Giaretto, S. Caron, A. Tona, A Novel zT Meter Based on the Porcupine Method and a Survey on the Size of the Snout Correction Needed for Various Thermoelectric Devices, J. Electron. Mater. 42 (2013) 2067-2072. https://doi.org/10.1007/s11664-013-2530-2.

[2] A. De Marchi, V. Giaretto, An accurate new method to measure the dimensionless figure of merit of thermoelectric devices based on the complex impedance porcupine diagram, Rev. Sci. Instrum. 82 (2011). https://doi.org/10.1063/1.3656074.

[3] Y. Hasegawa, R. Homma, M. Ohtsuka, Thermoelectric Module Performance Estimation Based on Impedance Spectroscopy, J. Electron. Mater. 45 (2016) 1886-1893. https://doi.org/10.1007/s11664-015-4271-x.

[4] M. Otsuka, H. Terakado, R. Homma, Y. Hasegawa, M.Z. Islam, G. Bastian, A. Stuck, Thermal diffusivity measurement using thermographic method and performance evaluation by impedance spectroscopy for thermoelectric module, in: Jpn. J. Appl. Phys., Japan Society of Applied Physics, 2016. https://doi.org/10.7567/JJAP.55.126601.

[5] J. García-cañadas, G. Min, Impedance spectroscopy models for the complete characterization of thermoelectric materials, J. Appl. Phys. 116 (2014). https://doi.org/10.1063/1.4901213.

[6] R. Mesalam, H.R. Williams, R.M. Ambrosi, J. García-Cañadas, K. Stephenson, Towards a comprehensive model for characterising and assessing thermoelectric modules by impedance spectroscopy, Appl. Energy. https://doi.org/10.1016/j.apenergy.2018.05.041.

[7] C.-Y. Yoo, C. Yeon, Y. Jin, Y. Kim, J. Song, H. Yoon, S.H. Park, B. Beltrán-Pitarch, J. García-Cañadas, G. Min, Determination of the thermoelectric properties of a skutteruditebased device at practical operating temperatures by impedance spectroscopy, Appl. Energy. 251 (2019) 113341. https://doi.org/10.1016/J.APENERGY.2019.113341.

[8] C.Y. Yoo, Y. Kim, J. Hwang, H. Yoon, B.J. Cho, G. Min, S.H. Park, Impedance spectroscopy for assessment of thermoelectric module properties under a practical 
$\begin{array}{lllll}\text { operating } & \text { temperature, } & \text { Energy. } & 152 & \text { (2018) }\end{array}$ https://doi.org/10.1016/j.energy.2017.12.014.

[9] F. Casalegno, A. De Marchi, V. Giaretto, Frequency domain analysis of spreadingconstriction thermal impedance, Rev. Sci. Instrum. $84 \quad$ (2013) 024901. https://doi.org/10.1063/1.4789765.

[10] B. Beltrán-Pitarch, J. García-Cañadas, Influence of convection at outer ceramic surfaces on the characterization of thermoelectric modules by impedance spectroscopy, J. Appl. Phys. 123 (2018). https://doi.org/10.1063/1.5019881.

[11] A.D. Downey, T.P. Hogan, B. Cook, Characterization of thermoelectric elements and devices by impedance spectroscopy, Rev. Sci. Instrum. 78 (2007) 093904. https://doi.org/10.1063/1.2775432.

[12] R. Mesalam, H. Williams, R. Ambrosi, D. Kramer, C. Barklay, K. Stephenson, Impedance Spectroscopy: A Tool for Assessing Thermoelectric Modules for Radioisotope Power Systems, in: IEEE Aerosp. Conf. Proc., IEEE Computer Society, 2019. https://doi.org/10.1109/AERO.2019.8741526.

[13] B. Beltrán-Pitarch, F. Vidan, J. García-Cañadas, Characterization of thermal contacts between heat exchangers and a thermoelectric module by impedance spectroscopy, Appl. $\begin{array}{lll}\text { Therm. } & \text { Eng. } & 114361 .\end{array}$ https://doi.org/10.1016/J.APPLTHERMALENG.2019.114361.

[14] Y. Muzychka, R. Culham, M. Yovanovich, Thermal Spreading Resistances in Rectangular Flux Channels: Part I Geometric Equivalences, in: American Institute of Aeronautics and Astronautics (AIAA), 2012. https://doi.org/10.2514/6.2003-4187.

[15] M. Yovanovich, C. Tien, G. Schneider, General solution of constriction resistance within a compound disk, in: 17th Aerosp. Sci. Meet., American Institute of Aeronautics and Astronautics, Reston, Virigina, 1979. https://doi.org/10.2514/6.1979-178.

[16] D. Maillet, S. André, J.-C. Batsale, A. Degiovanni, C. Moyne, Thermal Quadrupoles: Solving the Heat Equation through Integral Transforms, John Wiley and Sons, New-York, 2000. 
[17] B. Beltrán-Pitarch, J. Prado-Gonjal, A. V. Powell, P. Ziolkowski, J. García-Cañadas, Thermal conductivity, electrical resistivity, and dimensionless figure of merit (ZT) determination of thermoelectric materials by impedance spectroscopy up to $250{ }^{\circ} \mathrm{C}$, J. Appl. Phys. 124 (2018) 025105. https://doi.org/10.1063/1.5036937.

[18] B. Beltrán-Pitarch, J. Prado-Gonjal, A. V. Powell, J. García-Cañadas, Experimental conditions required for accurate measurements of electrical resistivity, thermal conductivity, and dimensionless figure of merit (ZT) using Harman and impedance spectroscopy methods, J. Appl. Phys. $125 \quad$ (2019) 025111. https://doi.org/10.1063/1.5077071.

[19] J. García-Cañadas, Chapter 7. Thermoelectric Modules: Power Output, Efficiency, and Characterization, in: Thermoelectr. Energy Convers. Basic Concepts Device Appl., Wiley-VCH Verlag GmbH \& Co. KGaA, 2017: pp. 127-146. https://doi.org/10.1002/9783527698110.ch7. 


\section{Final conclusions}




\section{Final conclusions}

The main objective of this project was to provide new developments and improvements for the advanced characterization of thermoelectric (TE) materials and devices by means of impedance spectroscopy (IS). This objective has been achieved, and the principal attainments are, as follows:

1. The possibility to determine the electrical resistivity $\rho_{T E}$, thermal conductivity $\lambda_{T E}$ (if the Seebeck coefficient $S$ is known), and the dimensionless figure of merit $z T$ of a bulk TE material by IS has been demonstrated for a skutterudite material, which shows low performance at room temperature, up to $250^{\circ} \mathrm{C}$. A clear impedance signal and suitable characterization were obtained even at the lowest temperature. It was found important to optimize the ac current amplitude in the impedance experiments. Random errors $<4.5 \%$ for all properties were identified by performing five measurements at each temperature with remade contacts. Systematic errors were also determined by comparison with commercial equipment measurements, resulting in values $<4 \%$, between $4 \%$ and $6 \%$, and $<8 \%$, for $\lambda_{T E}, \rho_{T E}$ and $z T$, respectively (see Chapter 3 ).

2. The ability to perform a complete characterization of all TE properties of a bulk skutterudite sample, which shows low performance at room temperature, from a single IS measurement, has been demonstrated up to $250{ }^{\circ} \mathrm{C}$. Random errors $\approx 5.5 \%,<13 \%$, $<2.5 \%$, and between 4 and $7 \%$ for $S, \lambda_{T E}, \rho_{T E}$, and $z T$, respectively, were obtained by performing five measurements at each temperature remaking the contacts. The random errors in the determination of the thermal conductivity are higher than the ones obtained in the previous approach due to the quadratic dependence of this property with the Seebeck coefficient. Systematic errors were also calculated by comparison with results from commercial equipment, resulting in errors $<2.5 \%$, $<5.5 \%$, between 5 and $9 \%$, and $<9 \%$ for the $S, \lambda_{T E}, \rho_{T E}$, and $z T$, respectively (see Chapter 4).

3. A new theoretical model for the IS measurements of TE modules under suspended conditions, which includes the convection effect at the outer surface of the external ceramics, has been developed. The theoretical model was experimentally validated by performing IS measurements under high vacuum and at room conditions to a 
commercial TE device. The experimental measurements allowed the determination of the convection heat transfer coefficient $h$ (if the Seebeck coefficient of the TE module is known), which opens up the possibility to develop TE modules as $h$ sensors. On the other hand, if $h$ is known, all the properties of the TE module $\left(Z_{m} T\right.$, ohmic resistance, thermal conductivity of the ceramics, average Seebeck coefficient, and average thermal conductivity of the thermoelements) can be obtained from one impedance measurement in vacuum and another measurement at room conditions. A final analysis also showed that a $6 \%$ underestimation of $Z_{m} T$ can be produced if the new equivalent circuit is not employed when the characterization of TE modules is performed at room conditions (see Chapter 5).

4. To study the effect of a thermal contact resistance in the impedance signal of a TE module, a new theoretical model (equivalent circuit) has been developed considering a TE device sandwiched between heat exchangers working as ideal heat sinks. The theoretical model was tested by experimental measurements performed to a commercial Bi-Te TE module contacted by large aluminum blocks. It was found possible to quantify the value of the thermal contact resistivity between the TE module and the aluminum blocks. This can be performed from a measurement of the module in vacuum and the knowledge of the average Seebeck coefficient before being measured in contact with the blocks. The thermal contact resistivity values determined at different conditions (with and without thermal interface material and at different pressure levels) agree with literature values, opening up the possibility of using IS as a tool to quantify and monitor this parameter (see Chapter 6).

5. The addition and study of the effect of the thermal contact resistance between the TE legs and the metallic strips, the metallic strips themselves, and the thermal contact resistance between the metallic strips and the ceramic layer of a TE module, led to a new more comprehensive equivalent circuit that include all the key TE phenomena. Moreover, a new spreading-constriction impedance element, which considers the variation of the heat flow in the radial direction at the outer ceramic surfaces, has been obtained. It has been found that all the effects modify the impedance spectra at high frequencies, complicating the use of fittings to quantify the properties of the system. However, with the aid of IS simulations, a qualitative analysis of different TE modules 


\section{Chapter 8}

fabricated by different manufacturers has been performed. With the use of this equivalent circuit and the knowledge created by its analysis, the possibility of using IS as a quality control tool to monitor issues in TE devices is demonstrated (see Chapter 7). 


\section{Final conclusions (Spanish version)}

El objetivo principal de este proyecto fue proporcionar nuevos desarrollos y mejoras para la caracterización avanzada de materiales y dispositivos termoeléctricos (TEs) mediante espectroscopía de impedancia. Este objetivo se ha conseguido y los principales logros son los siguientes:

1. La posibilidad de determinar la resistividad eléctrica $\rho_{T E}$, la conductividad térmica $\lambda_{T E}$ (si se conoce el coeficiente Seebeck $S$ ), y la figura de mérito adimensional $z T$ de una escuterudita, que muestra baja eficiencia a temperatura ambiente, por espectroscopía de impedancia se ha demostrado hasta $250{ }^{\circ} \mathrm{C}$. Se obtuvo una señal de impedancia clara y una caracterización adecuada incluso a la temperatura más baja. Se descubrió que era importante optimizar la amplitud de corriente ac que se emplea en las medidas de impedancia. Se identificaron errores aleatorios $<4.5 \%$ para todas las propiedades TEs, realizando cinco medidas a cada temperatura rehaciendo los contactos. Los errores sistemáticos se determinaron por comparación con medidas de equipos comerciales, lo que resultó en valores $<4 \%$, entre $4 \%$ y $6 \%, \mathrm{y}<8 \%$, para $\lambda_{T E}, \rho_{T E} \mathrm{y} z T$, respectivamente (ver Capítulo 3).

2. La capacidad de realizar una caracterización completa de todas las propiedades TEs de una escuterudita, que muestra baja eficiencia a temperatura ambiente, a partir de una única medida de espectroscopía de impedancia, se ha demostrado hasta $250{ }^{\circ} \mathrm{C}$. Se obtuvieron errores aleatorios $\approx 5.5 \%,<13 \%,<2.5 \%$ y entre 4 y $7 \%$ para $S, \lambda_{T E}, \rho_{T E}$ y $z T$, respectivamente, realizando cinco medidas (rehaciendo los contactos) a cada temperatura. Los errores aleatorios en la determinación de la conductividad térmica son mayores que los obtenidos en el método anterior debido a la dependencia cuadrática de esta propiedad con el coeficiente Seebeck. Los errores sistemáticos también se calcularon por comparación con resultados de equipos comerciales, lo que resultó en errores $<2.5 \%,<5.5 \%$, entre 5 y $9 \%$ y $<9 \%$ para $S, \lambda_{T E}, \rho_{T E}$ y $z T$, respectivamente (ver Capítulo 4).

3. Se ha desarrollado un nuevo modelo teórico para medidas de espectroscopía de impedancia a módulos TEs en condiciones suspendidas, que incluye el efecto de convección en la superficie externa de las cerámicas del módulo. El modelo teórico 
fue validado experimentalmente realizando medidas de espectroscopía de impedancia bajo alto vacío y a presión ambiente a un dispositivo TE comercial. Las medidas experimentales permitieron la determinación del coeficiente de transferencia de calor por convección $h$ (si se conoce el coeficiente Seebeck del módulo TE), lo que abre la posibilidad de desarrollar módulos TEs como sensores de $h$. Por otro lado, si se conoce $h$, todas las propiedades del módulo TE $\left(Z_{m} T\right.$, resistencia óhmica, conductividad térmica de las cerámicas, coeficiente Seebeck promedio y conductividad térmica promedio de los elementos TE) se pueden obtener con una medida de impedancia en vacío y otra medida en condiciones ambientales. Un análisis final también mostró que se puede producir una subestimación del $6 \%$ en la $Z_{m} T$ si el nuevo circuito equivalente no se emplea cuando la caracterización de los módulos TEs se realiza en condiciones ambientales (ver Capítulo 5).

4. Para estudiar el efecto de una resistencia térmica de contacto en la señal de impedancia de un módulo TE, se ha desarrollado un nuevo modelo teórico (circuito equivalente) considerando un dispositivo TE intercalado entre intercambiadores de calor que actúan como disipadores de calor ideales. El modelo teórico se probó mediante medidas experimentales realizadas en un módulo TE comercial en contacto con dos bloques de aluminio. Fue posible cuantificar el valor de la resistividad térmica de contacto entre el módulo TE y los bloques de aluminio. Esto se puede realizar a partir de una medida del módulo en vacío y con el conocimiento del coeficiente Seebeck promedio antes de medir el módulo en contacto con los bloques. Los valores de resistividad térmica de contacto determinados en diferentes condiciones (con y sin material de mejora de contacto térmico y a diferentes niveles de presión de contacto) concuerdan con los valores de la literatura, lo que abre la posibilidad de utilizar la espectroscopía de impedancia como una herramienta para cuantificar y monitorizar este parámetro (ver Capítulo 6).

5. La adición y el estudio del efecto de la resistencia térmica de contacto entre los elementos TEs y las tiras metálicas, las tiras metálicas en sí mismas, y la resistencia térmica de contacto entre las tiras metálicas y las cerámicas de un módulo TE, condujeron a un nuevo circuito equivalente más completo que incluye todos los fenómenos TEs clave. Además, se ha obtenido un nuevo elemento de impedancia 


\section{Chapter 8}

debido a la expansión y constricción del calor al pasar de una superficie a otra de área diferente que considera la variación del flujo de calor en la dirección radial en las superficies cerámicas externas. Se ha visto que todos los efectos modifican los espectros de impedancia a altas frecuencias, lo que complica el uso de ajustes para cuantificar las propiedades del sistema. Sin embargo, con la ayuda de simulaciones de los espectros de impedancia, se realizó un análisis cualitativo de diferentes módulos TEs fabricados por diferentes fabricantes. Con el uso de este circuito equivalente y el conocimiento creado por su análisis, se demuestra la posibilidad de usar la espectroscopía de impedancia como una herramienta de control de calidad para monitorizar problemas en dispositivos TEs (ver Capítulo 7). 


\section{Final conclusions (Valencian version)}

L'objectiu principal d'aquest projecte ha sigut proporcionar nous desenvolupaments i millores per a la caracterització avançada de materials i dispositius termoelèctrics (TEs) mitjançant l'espectroscòpia d'impedància. Aquest objectiu s'ha aconseguit i els principals assoliments són els següients:

1. La possibilitat de determinar la resistivitat elèctrica $\rho_{T E}$, la conductivitat tèrmica $\lambda_{T E}$ (si es coneix el coeficient Seebeck $S$ ), i la figura de mèrit adimensional $z T$ d'una escuterudita, la qual mostra baixa eficiència a temperatura ambient, per espectroscòpia d'impedància s'ha demostrat fins a $250^{\circ} \mathrm{C}$. Es va obtindre un senyal d'impedància clara i una caracterització adequada fins i tot a la temperatura més baixa. Es va descobrir que era important optimitzar l'amplitud de corrent ac que s'empra en les mesures d'impedància. Es van identificar errors aleatoris $<4.5 \%$ per a totes les propietats TEs, realitzant cinc mesures a cada temperatura refent els contactes. Els errors sistemàtics es van determinar per comparació amb mesures d'equips comercials, la qual cosa va resultar en valors $<4 \%$, entre $4 \% \mathrm{i} 6 \%, \mathrm{i}<8 \%$, per a $\lambda_{T E}, \rho_{T E} \mathrm{i} z T$, respectivament (veure Capítol 3).

2. La capacitat de realitzar una caracterització completa de totes les propietats TEs d'una escuterudita, que mostra baixa eficiència a temperatura ambient, a partir d'una única mesura d'espectroscòpia d'impedància, s'ha demostrat fins a $250{ }^{\circ} \mathrm{C}$. Es van obtindre errors aleatoris $\approx 5.5 \%,<13 \%,<2.5 \%$ i entre $4 \mathrm{i} 7 \%$ per a $S, \lambda_{T E}, \rho_{T E}$ i $z T$, respectivament i es van realitzar cinc mesures (fent els contactes de nou abans de mesurar) a cada temperatura. Els errors aleatoris en la determinació de la conductivitat tèrmica són majors que els obtinguts en el mètode anterior a causa de la dependència quadràtica d'aquesta propietat amb el coeficient Seebeck. Els errors sistemàtics també es van calcular per comparació amb resultats d'equips comercials i es van obtenir errors $<2.5 \%,<5.5 \%$, entre $5 \mathrm{i} 9 \%$ i $<9 \%$ per a $S, \lambda_{T E}, \rho_{T E}$ i $z T$, respectivament (veure Capítol 4).

3. S'ha desenvolupat un nou model teòric per a mesures d'espectroscòpia d'impedància a mòduls TEs en condicions suspeses, que inclou l'efecte de convecció en la superfície externa de les ceràmiques del mòdul. El model teòric va ser validat experimentalment 
realitzant mesures d'espectroscòpia d'impedància en buit i a pressió ambient a un dispositiu TE comercial. Les mesures experimentals van permetre la determinació del coeficient de transferència de calor per convecció $h$ (si es coneix el coeficient Seebeck del mòdul TE), la qual cosa obri la possibilitat de desenvolupar mòduls TEs com a sensors d'h. D'altra banda, si es coneix $h$, totes les propietats del mòdul TE $\left(Z_{m} T\right.$, resistència òhmica, conductivitat tèrmica de les ceràmiques, coeficient Seebeck mitjà i conductivitat tèrmica mitjana dels elements TEs) es poden obtindre amb una mesura d'impedància en buit i una altra mesura en condicions ambient. Una anàlisi final també va mostrar que es pot produir una subestimació del $6 \%$ en la $Z_{m} T$ si el nou circuit equivalent no s'empra quan la caracterització dels mòduls TE es realitza en condicions ambient (veure Capítol 5).

4. Per a estudiar l'efecte d'una resistència tèrmica de contacte en el senyal d'impedància d'un mòdul TE, s'ha desenvolupat un nou model teòric (circuit equivalent) que considera un dispositiu TE intercalat entre intercanviadors de calor que actuen com a dissipadors de calor ideals. El model teòric es va provar mitjançant mesures experimentals realitzades amb un mòdul TE comercial en contacte amb dos blocs d'alumini. Va ser possible quantificar el valor de la resistivitat tèrmica de contacte entre el mòdul TE i els blocs d'alumini. Això es pot realitzar a partir d'una mesura del mòdul en buit i amb el coneixement del coeficient Seebeck mitjà abans de mesurar el mòdul en contacte amb els blocs. Els valors de resistivitat tèrmica de contacte determinats en diferents condicions (amb i sense material de millora de contacte tèrmic i a diferents nivells de pressió de contacte) concorden amb els valors de la literatura, la qual cosa obri la possibilitat d'utilitzar l'espectroscòpia d'impedància com una eina per a quantificar i monitoritzar aquest paràmetre (veure Capítol 6).

5. L'addició i l'estudi de l'efecte de la resistència tèrmica de contacte entre els elements TEs i les tires metàl-liques, les tires metàl-liques en si mateixes, i la resistència tèrmica de contacte entre les tires metàl-liques i les ceràmiques d'un mòdul TE, van conduir a un nou circuit equivalent més complet que inclou tots els fenòmens TEs clau. A més a més, s'ha obtingut un nou element d'impedància a causa de l'expansió i constricció de la calor en passar d'una superfície a una altra d'àrea diferent que considera la variació del flux de calor en la direcció radial en les superfícies ceràmiques externes. 


\section{Chapter 8}

S'ha vist que tots els efectes modifiquen els espectres d'impedància a altes freqüències, la qual cosa complica l'ús d'ajustos per a quantificar les propietats del sistema. No obstant això, amb l'ajuda de simulacions dels espectres d'impedància, es va realitzar una anàlisi qualitativa de diferents mòduls TEs fabricats per distints fabricants. Amb l'ús d'aquest circuit equivalent i el coneixement creat per la seua anàlisi, es demostra la possibilitat d'usar l'espectroscòpia d'impedància com una eina de control de qualitat per a monitoritzar problemes en dispositius TEs (veure Capítol 7). 
9. Annexes 


\subsection{Code used in Chapter 6}

In this annex, it is provided the Matlab code used in Chapter 6, which is capable of extracting the thermal contact resistance from an impedance spectroscopy (IS) measurement in suspended conditions and another with the thermoelectric (TE) module contacted with heat exchangers:

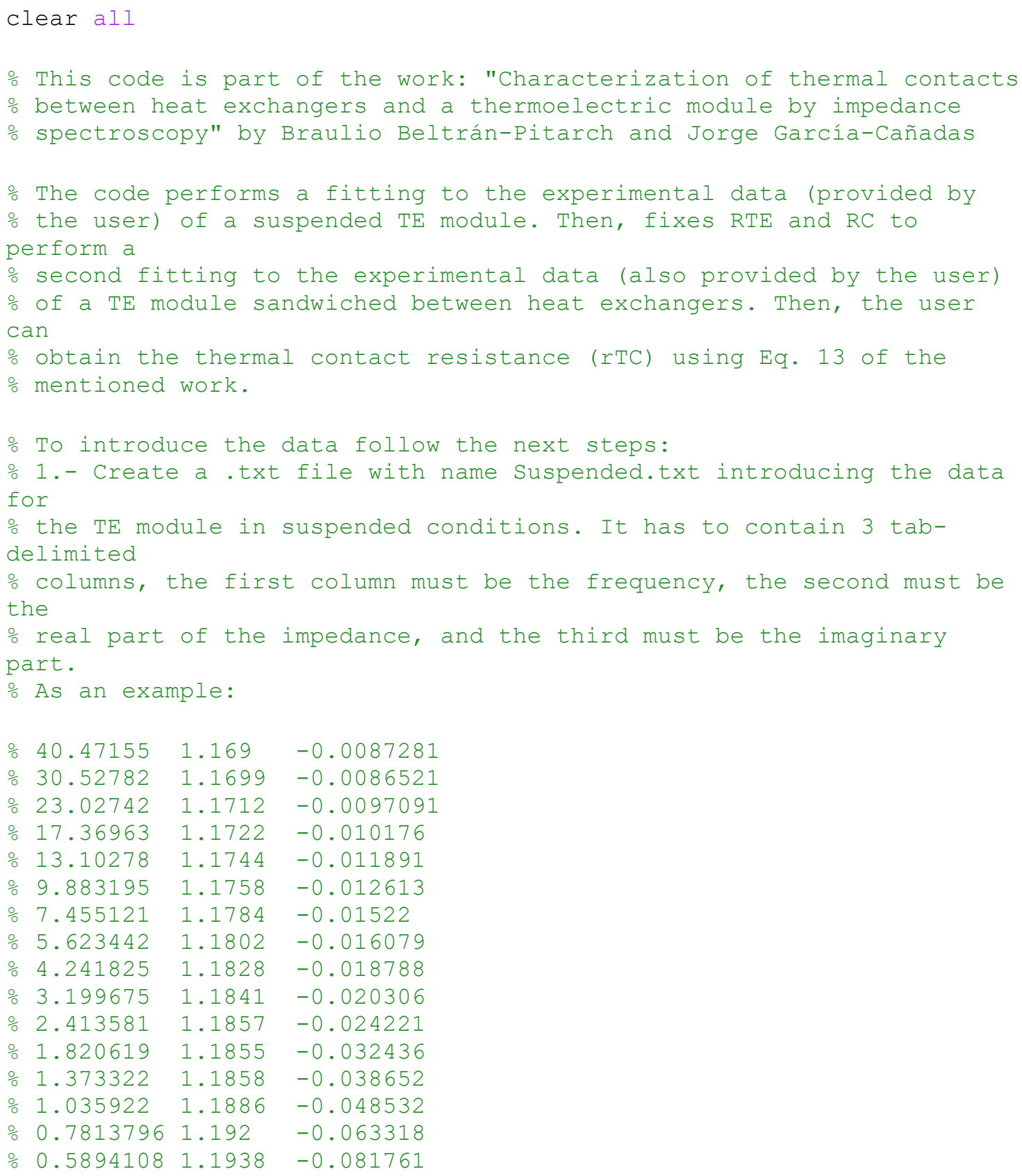




\section{Chapter 9}

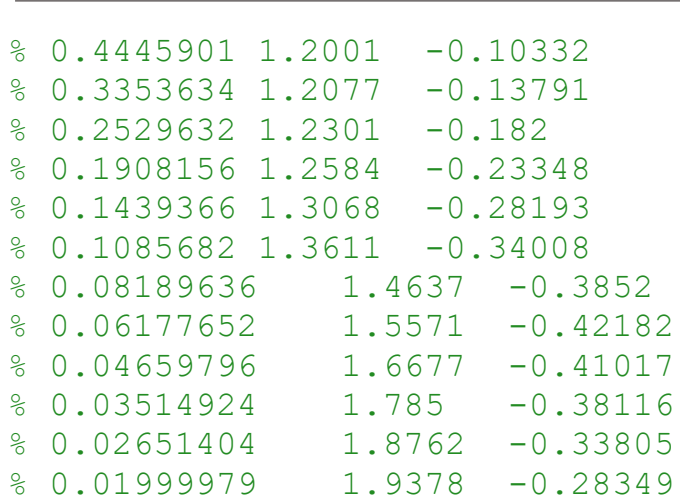

ㅇ 2.- Repeat the same for the module sandwiched between heat exchangers.

This time save the file as Exchangers.txt. As an example:

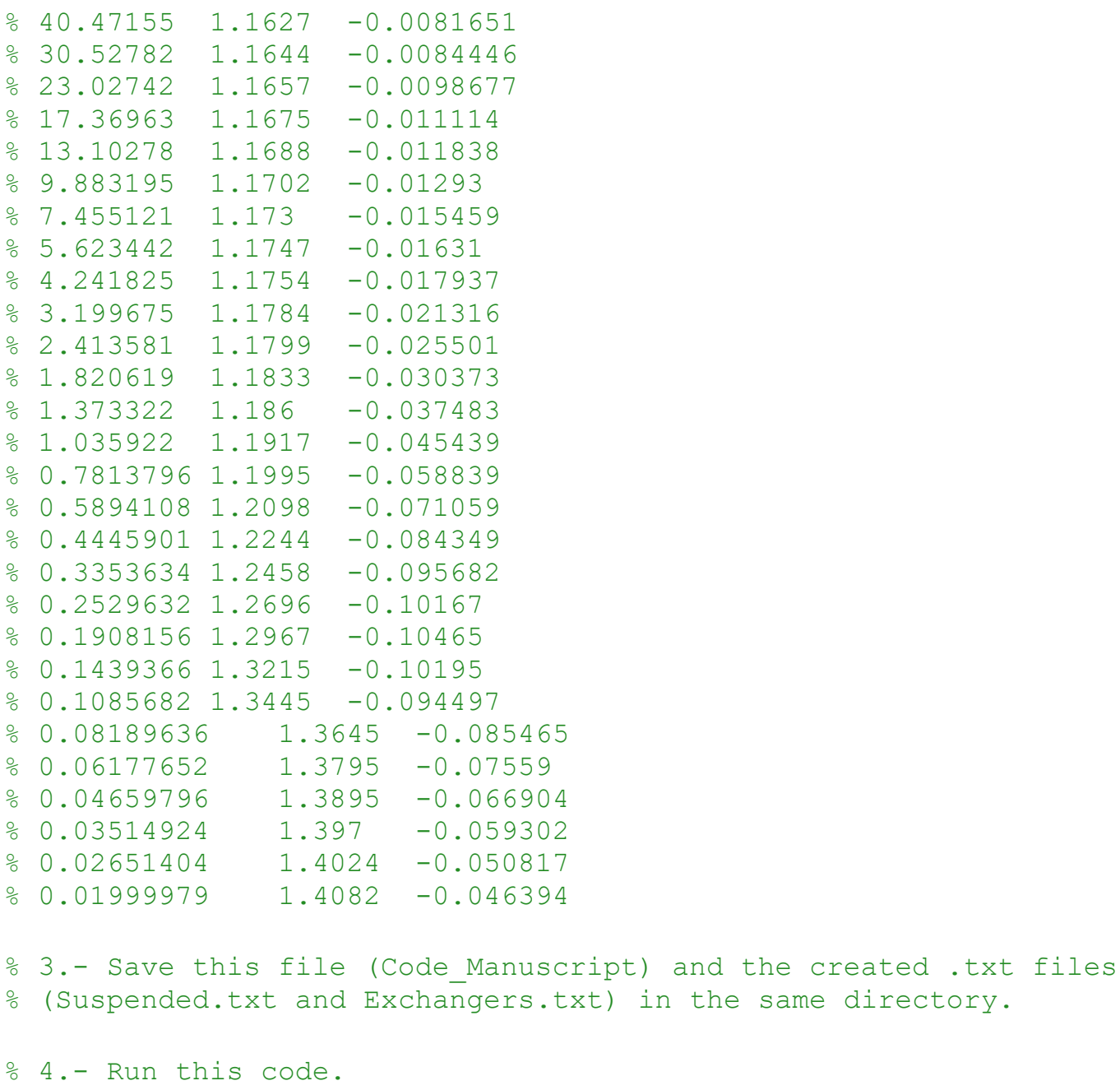




\section{Chapter 9}

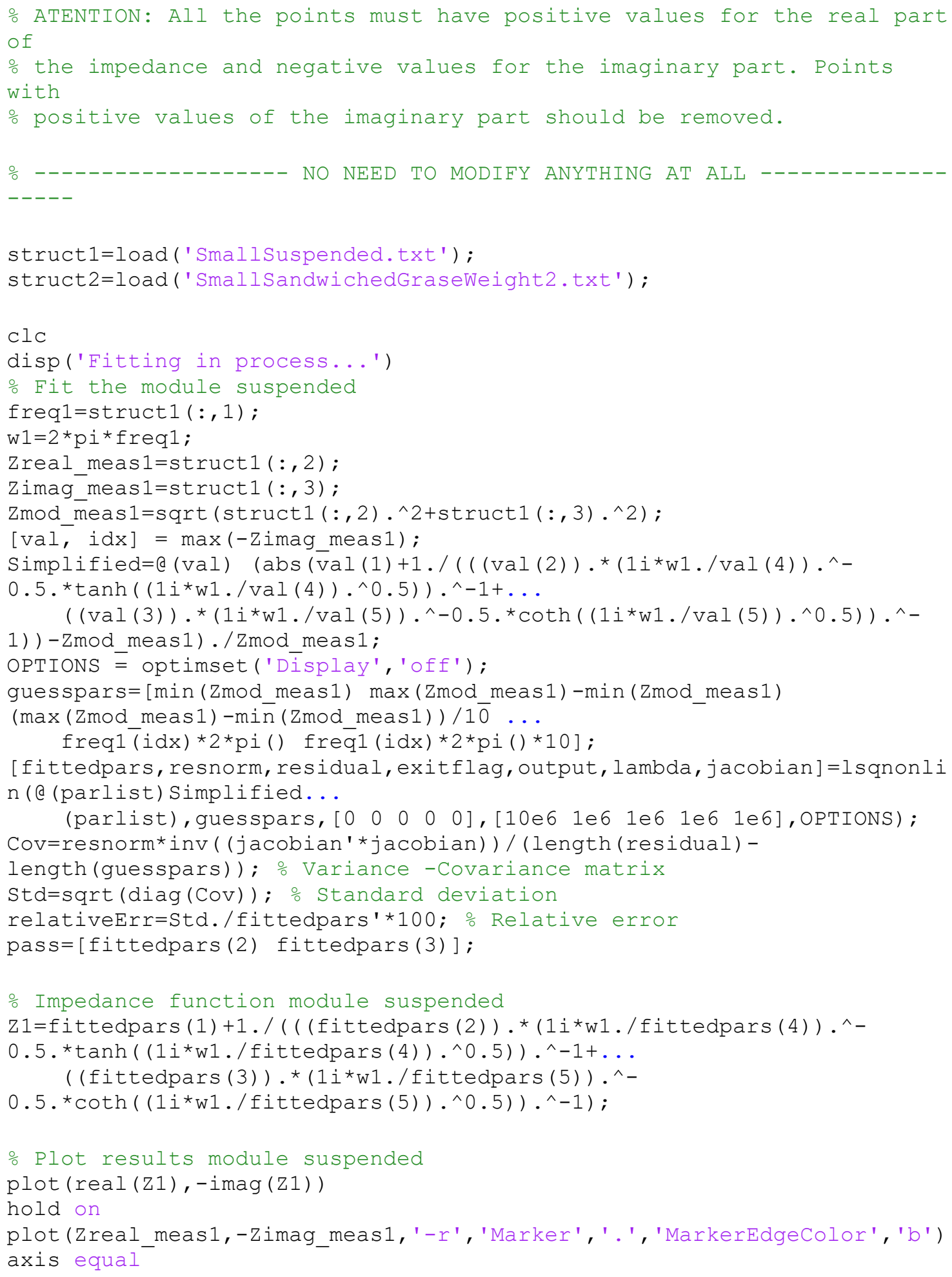




\section{Chapter 9}

hold off

title('TE module suspended')

legend('Fitting', 'Expermental data')

xlabel ('Real Z (\Omega)')

ylabel ('-Imag Z (\Omega)')

Clc

\% Show results module suspended

$\operatorname{disp}(' \quad$ ')

disp('RESULTS OF THE TE MODULE SUSPENDED:')

disp (' ')

disp(['Ohmic resistance (Rohm) = ' num2str(fittedpars(1)) ' Ohm' '

Error = ' num2str (relativeErr(1)) ' o '])

disp(['Thermoelectric resistance (RTE) = ' num2str(fittedpars(2)) '

Ohm' ' Error = ' num2str(relativeErr(2)) ' o'])

disp(['Contact resistance $(\mathrm{RC})=$ ' num2str(fittedpars(3)) ' Ohm' '

Error $=$ ' num2str(relativeErr(3)) ' \%'])

disp(['Thermoelectric characteristic angular frequency (wTE) = '

num2str(fittedpars (4)) ' $\mathrm{rad} / \mathrm{s}$ ' ' Error $=$ '

num2str(relativeErr(4)) ' \%'])

disp(['Ceramic characteristic angular frequency $(\mathrm{wC})=$ '

num2str(fittedpars(5)) ' rad/s' ' Error $=$ '

num2str(relativeErr(5)) ' 잉)

$\operatorname{disp}('$ ')

figure

o Fit the module with the heat exchangers

freq2 $=\operatorname{struct} 2(:, 1)$;

$\mathrm{w} 2=2 * \mathrm{pi}$ *req2;

Zreal_meas $2=\operatorname{struct} 2(:, 2)$;

Zimag_meas $2=\operatorname{struct} 2(:, 3)$;

Zmod meas $2=\operatorname{sqrt}\left(\operatorname{struct} 2(:, 2) \cdot{ }^{\wedge} 2+\operatorname{struct} 2(:, 3) . \wedge 2\right)$;

Simplified_pro=@ (valor)

(abs (valor $(1)+1 . /\left(\left((\operatorname{pass}(1)) \cdot{ }^{\star}\left(1 i{ }^{\star} w 2 . / \operatorname{valor}(3)\right) .^{\wedge}-\right.\right.$

$\left.0.5 .{ }^{\star} \tanh \left(\left(1 i{ }^{*} \mathrm{w} 2 . / \operatorname{valor}(3)\right) . \wedge 0.5\right)\right) \cdot{ }^{\wedge}-1+\ldots$

( ( ( (pass (2)) .* (1i*w2./valor (4)) .^-

$\left.0.5 .{ }^{*} \operatorname{coth}\left(\left(1{ }^{*}{ }^{*} 2 . / \operatorname{valor}(4)\right) \cdot{ }^{\wedge} 0.5\right)\right) \cdot{ }^{\wedge}-1+\ldots$

$\left.(\operatorname{valor}(2)) \cdot \cdot^{\wedge}-1\right) \cdot{ }^{\wedge}-1+\left(\left((\operatorname{pass}(2) \wedge 2 / \operatorname{valor}(2)) \cdot{ }^{\star}\left(1 i{ }^{\star} w 2 \cdot / \operatorname{valor}(4)\right) \cdot{ }^{\wedge}-\right.\right.$

$1) \cdot \wedge-1+\ldots$.

$\left((\operatorname{pass}(2)) \cdot{ }^{\star}(1 i * w 2 . / \operatorname{valor}(4)) \cdot^{\wedge}-\right.$

$\left.\left.\left.\left.\left.0.5 .{ }^{*} \tanh \left(\left(1{ }^{*}{ }^{*} 2 . / \operatorname{valor}(4)\right) \cdot{ }^{\wedge} 0.5\right)\right) \cdot{ }^{\wedge}-1\right) \cdot{ }^{\wedge}-1\right) \cdot{ }^{\wedge}-1\right)\right)-$

Zmod_meas2)./Zmod_meas2;

OPTIŌNS = optimset'('Display', 'off');

guesspars=[fittedpars(1) fittedpars(2)/10 fittedpars(4)

fittedpars (5)];

[fittedpars, resnorm, residual, exitflag, output, lambda, jacobian] =lsqnonli

n ( ( (parlist) Simplified_pro...

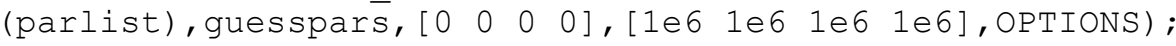

Cov=resnorm*inv ( (jacobian'*jacobian)) / (length (residual) -

length(guesspars)); Variance -Covariance matrix

Std=sqrt (diag (Cov)); $\frac{\circ}{0}$ Standard deviation 


\section{Chapter 9}

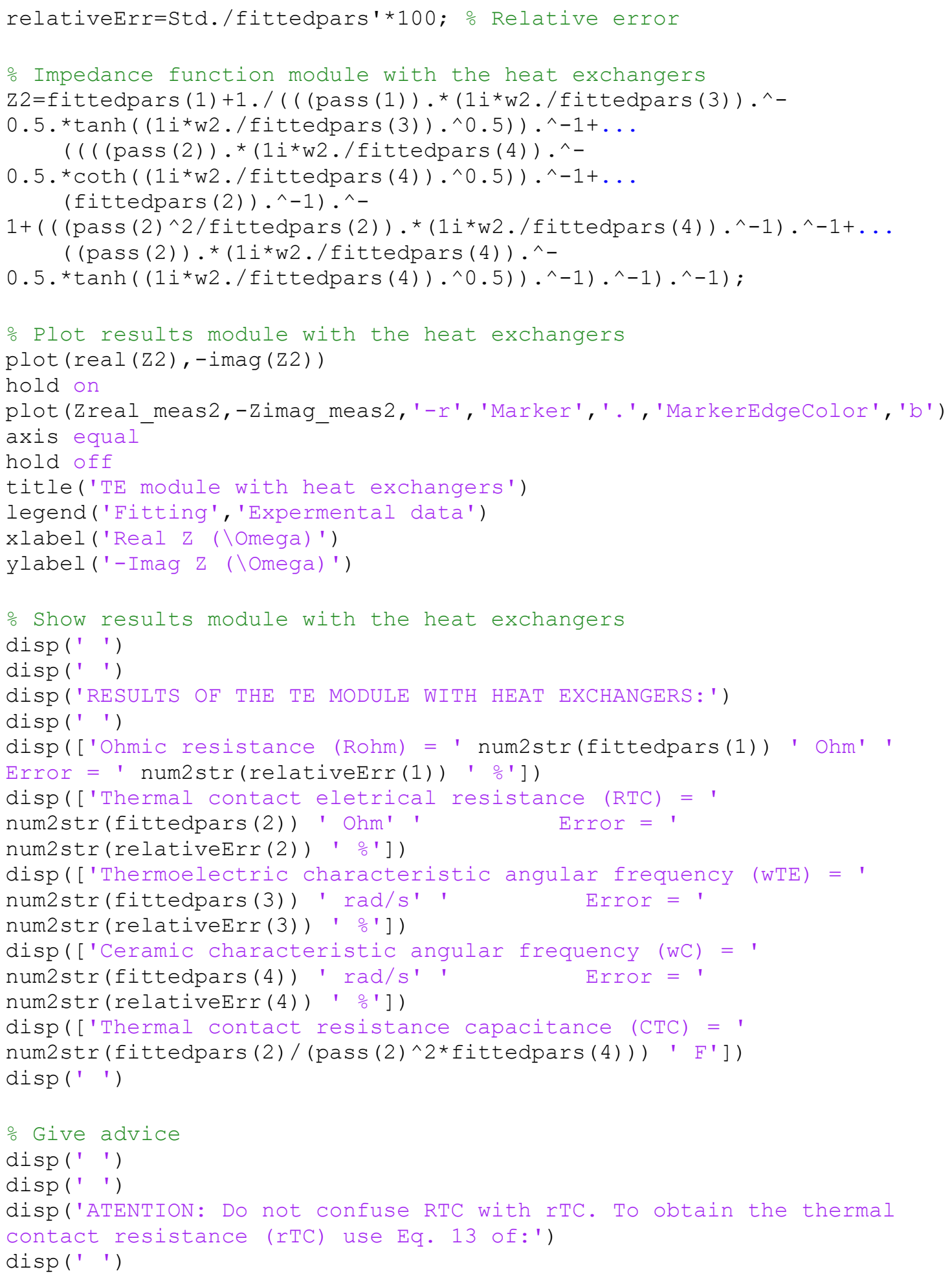




\section{Chapter 9}

disp('"Characterization of thermal contacts between heat exchangers and a thermoelectric module by impedance spectroscopy"') disp('by Braulio Beltrán-Pitarch and Jorge García-Cañadas') disp(' ')

disp('You will need:')

disp('Tinitial: Ambient temperature in K')

disp('N: Number of couples of the TE module')

disp('S: Average Seebeck coefficient of the TE legs')

disp('n: Filling factor of the TE module')

disp('A: Cross-sectional area of a TE leg')

disp('RTC: Thermal contact eletrical resistance (obtained in the second fitting)') 


\subsection{Code used in Chapter 7}

In this annex, it is provided the Matlab code used in Chapter 7, which can be used to simulate IS measurements to assess TE devices by comparison with experimental data:

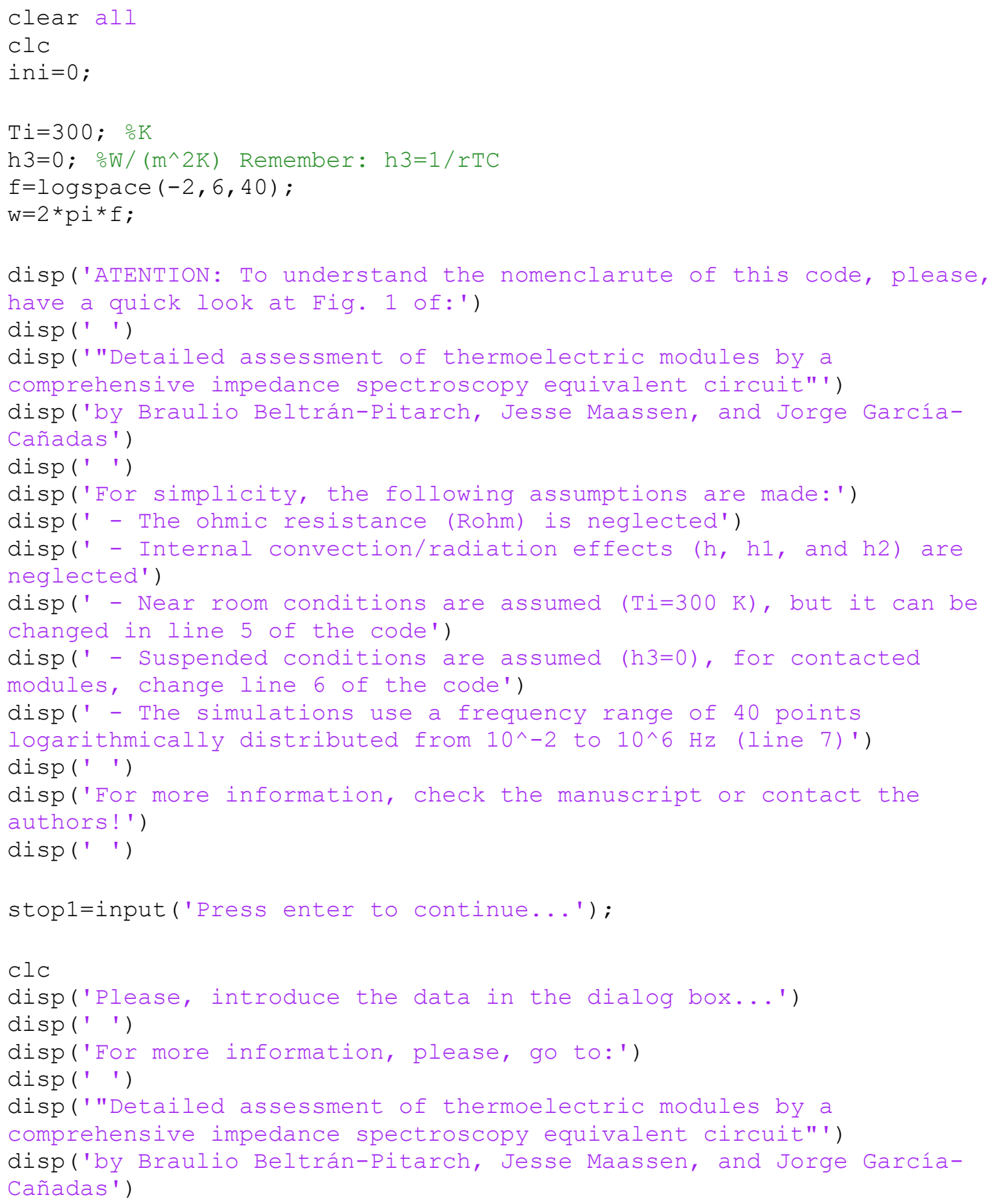




\section{Chapter 9}

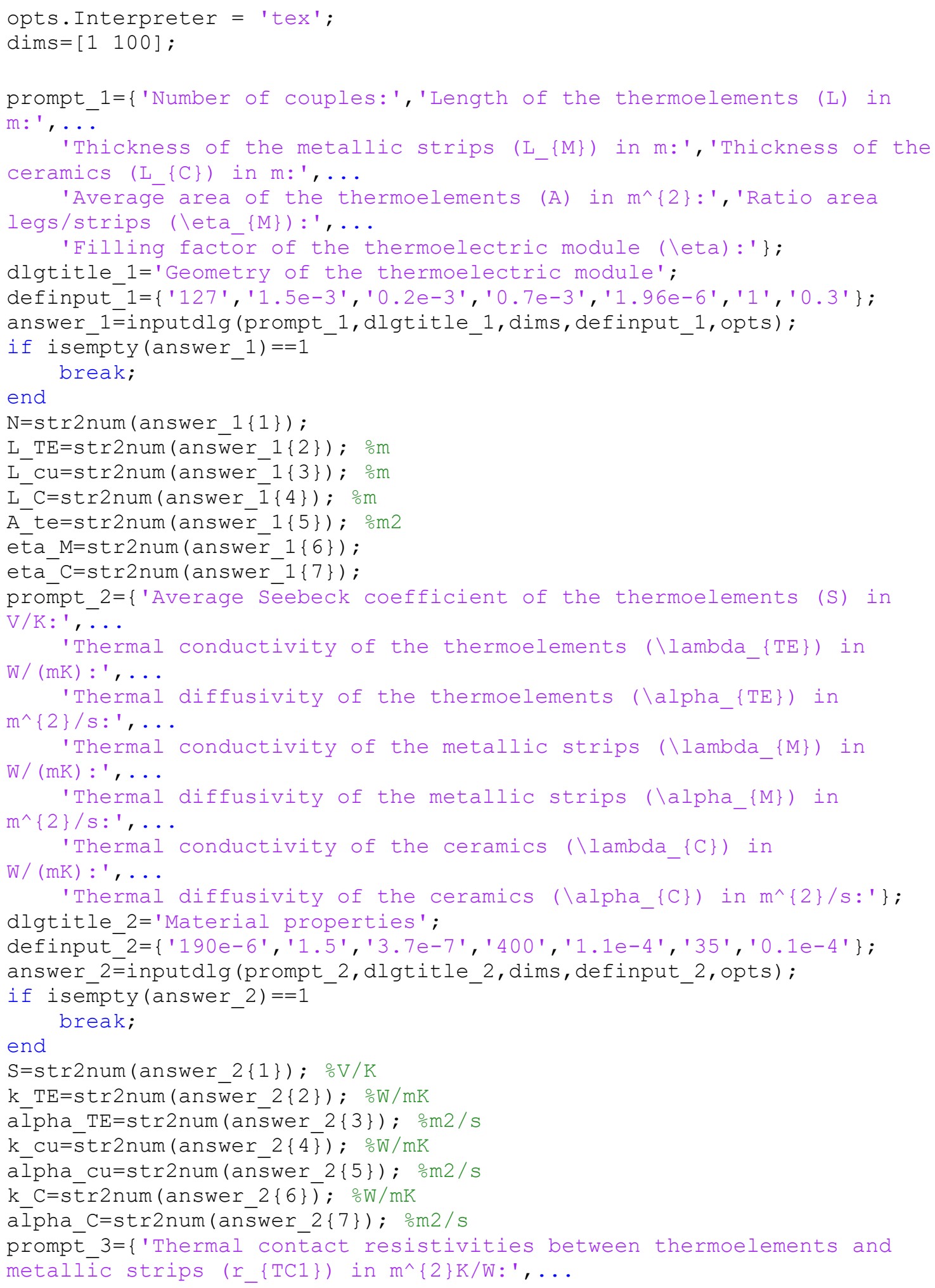




\section{Chapter 9}

'Thermal contact resistivities between metallic strips and ceramics (r $\{\mathrm{TC} 2\}$ ) in $\left.\mathrm{m}^{\wedge}\{2\} \mathrm{K} / \mathrm{W}: \mathbf{C}^{\prime}\right\}$;

dlgtitle_3='Thermal contact resistances';

definput $3=\left\{\right.$ ' $\left.^{0} 0,1 e-6,5 e-6,1 e-5\right]$ ' , ' $[0,1 e-6,5 e-6,1 e-5]$ ' $\}$;

answer_3=inputdlg (prompt_3,dlgtitle_3,dims,definput_3,opts) ;

if isempty (answer_3) $==1$

break;

end

$\mathrm{RC} 1=\operatorname{str} 2$ num (answer $3\{1\}) ; \%$ TC 1

$\mathrm{RC} 2=\operatorname{str} 2$ num (answer_3 $\{2\}) ; \%$ TTC2

while 1

if ini $>0$

$\mathrm{clc}$

disp('Please, introduce the data in the dialog box...')

$\operatorname{disp}('$ ' $)$

disp('For more information, please, go to:')

$\operatorname{disp}('$ ' $)$

disp("Detailed assessment of thermoelectric modules by a

comprehensive impedance spectroscopy equivalent circuit"')

disp('by Braulio Beltrán-Pitarch, Jesse Maassen, and Jorge

García-Cañadas' ')

prompt $1=\{$ 'Number of couples:', 'Length of the thermoelements

(L) in $m:$ ',...

'Thickness of the metallic strips ( $L\{M\})$ in

m:','Thickness of the ceramics ( L_ $\{\mathrm{C}\})$ in $m:{ }^{\prime}, \ldots$

'Average area of the thermoelements (A) in $m^{\wedge}\{2\}:$ ', 'Ratio

area legs/strips ( leta $\{\mathrm{M}\}):{ }^{\prime}, \ldots$

'Filling fáctor of the thermoelectric module ( leta):'\};

dlgtitle_1='Geometry of the thermoelectric module';

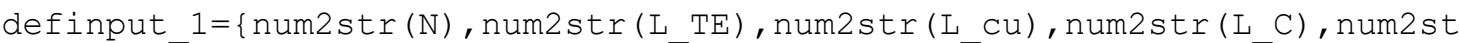
$r($ A_te), num2str (eta_M), num2str (eta_C) \};

answer_1=inputdlg (prompt_1,dlgtitle_1,dims,definput_1,opts) ;

if isempty (answer 1$)==1$

break;

end

$\mathrm{N}=$ str 2 num (answer $1\{1\})$;

L TE=str2num(answer $1\{2\}) ; \circ \mathrm{m}$

L_cu=str2num (answer_1\{3\}); $\frac{\mathrm{m}}{\mathrm{m}}$

$\mathrm{L}_{-} \mathrm{C}=\mathrm{str} 2 \mathrm{num}($ answer_ $\overline{1}\{4\}) ; \% \mathrm{~m}$

A te=str2num (answe $\bar{r} 1\{5\}) ; \circ \mathrm{m} 2$

eta_M=str2num(answer $1\{6\})$;

eta_C=str2num(answer_1\{7\});

prompt $2=\{$ 'Average Seebeck coefficient of the thermoelements

(S) in $V / K: ', \ldots$

'Thermal conductivity of the thermoelements (\lambda_\{TE\})

in $W /(m K): ', \ldots$

'Thermal diffusivity of the thermoelements ( \alpha $\{\mathrm{TE}\}$ )

in $m^{\wedge}\{2\} / s: ', \ldots$ 


\section{Chapter 9}

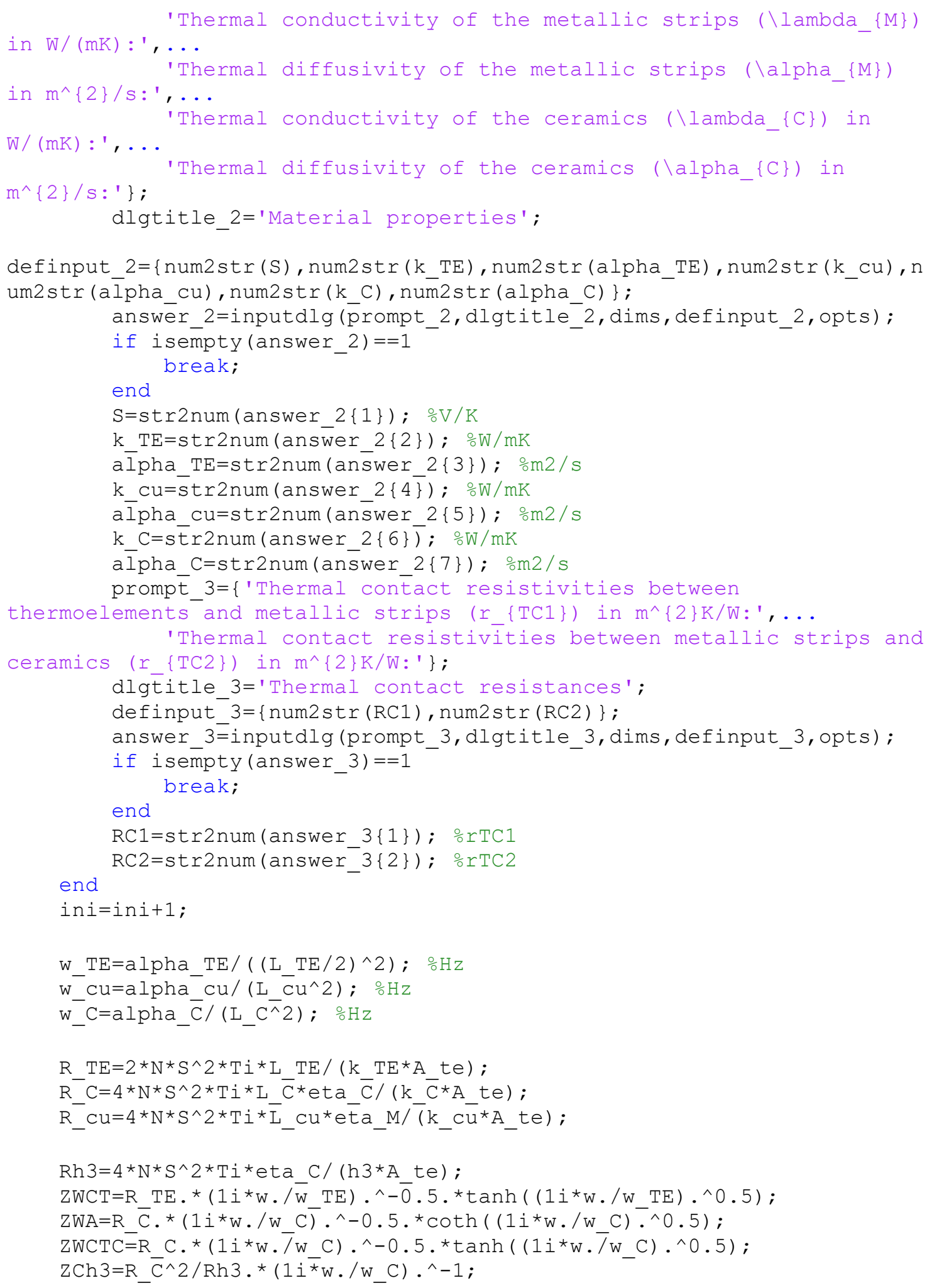




\section{Chapter 9}

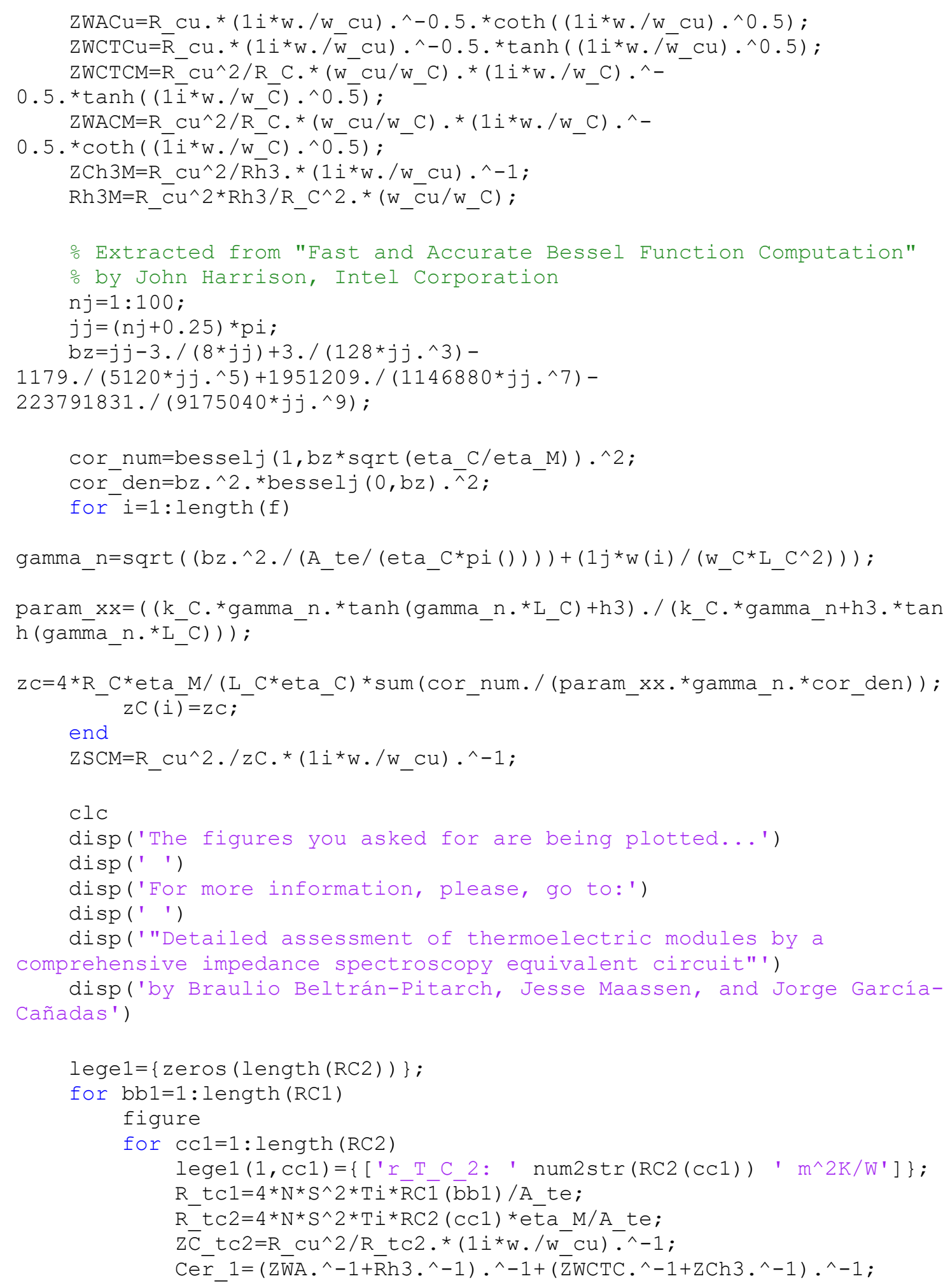




\section{Chapter 9}

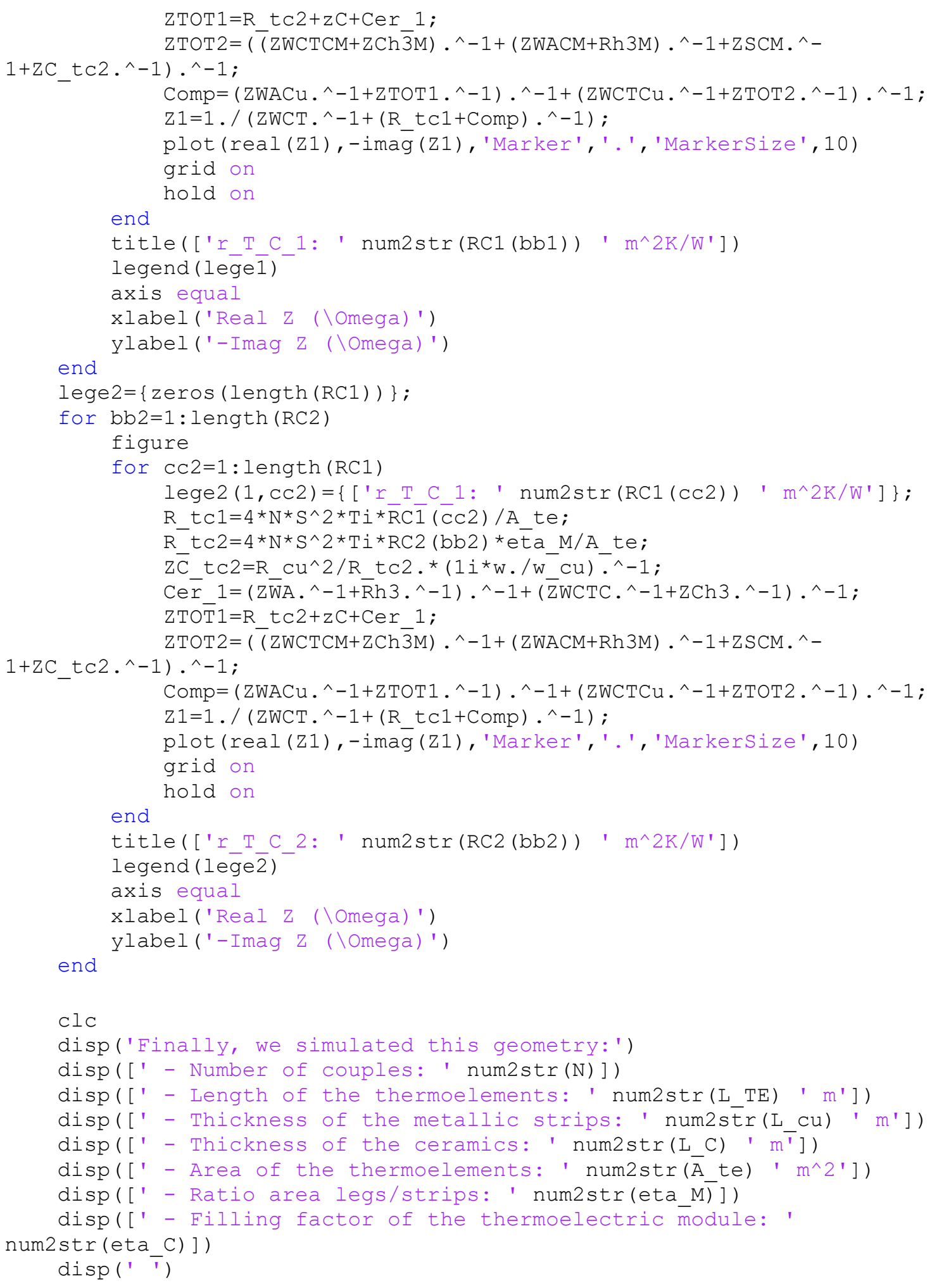




\section{Chapter 9}

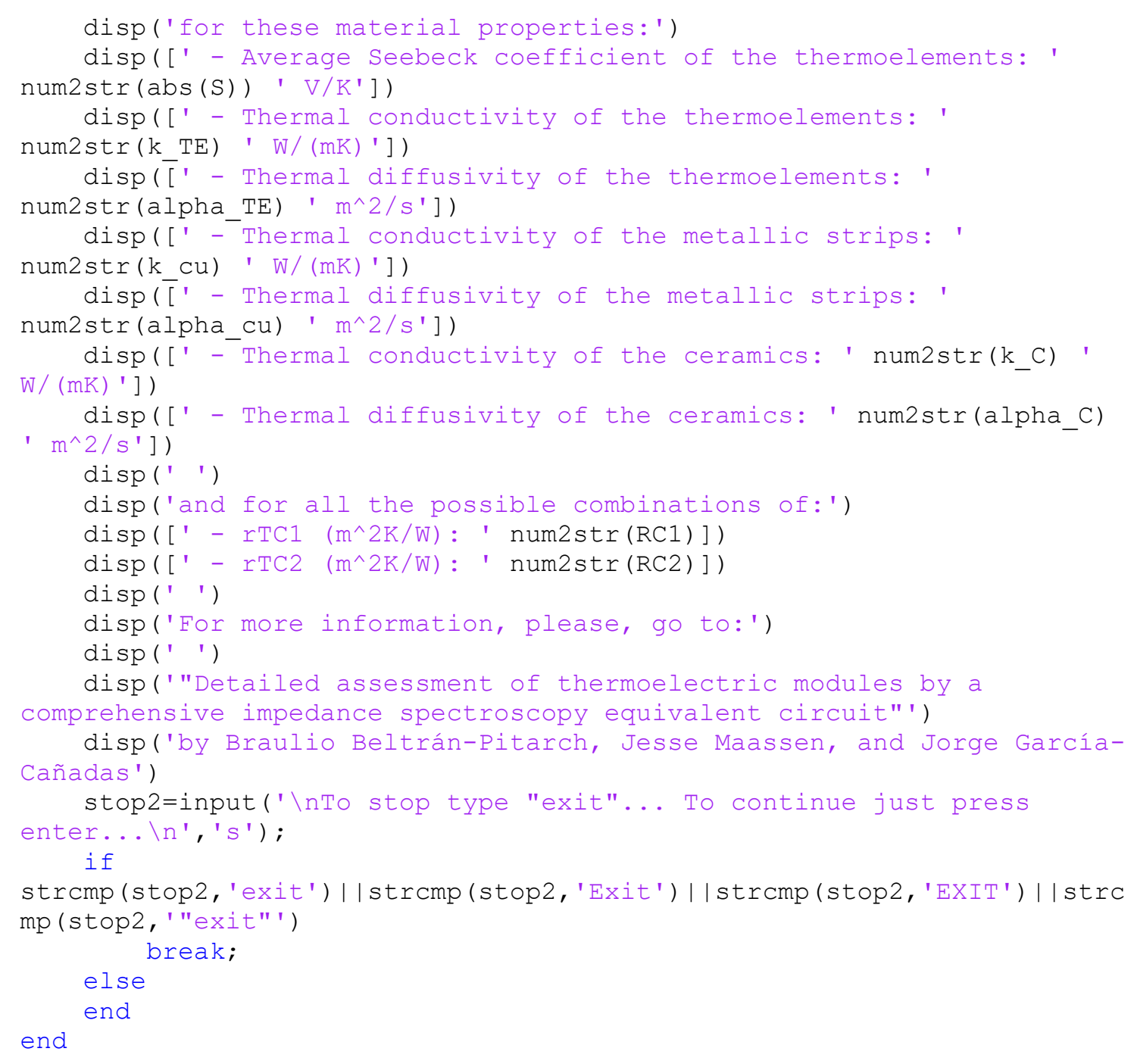




\subsection{Spreading-constriction derivations}

This annex presents the derivations to obtain the expression for the spreading-constriction impedance of Eq. (7.6). The theoretical model shown in Fig. S9.1 was considered. It consists of two cylindrical layers with different area, which simulate a metallic strip and a ceramic of a TE device. The metallic strip has a radius $r_{M}$ and applies a uniform heat flux $\varphi_{0}$ into the ceramic. The ceramic layer has a length $L_{C}$ and a radius $r_{C}$.

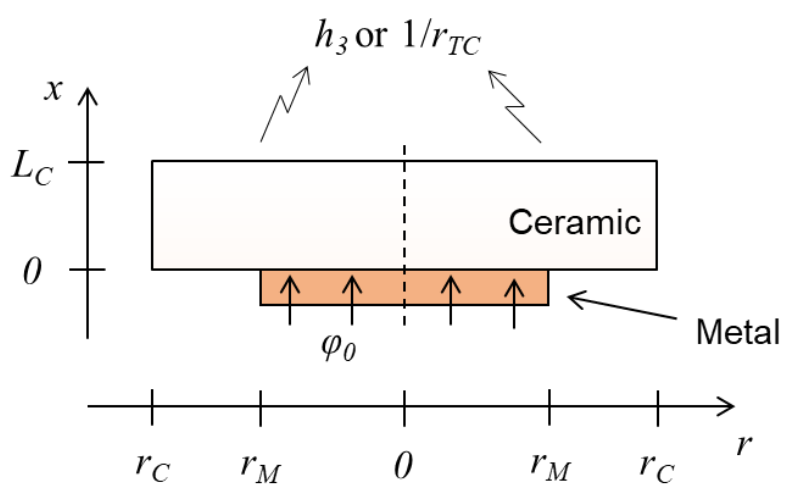

Fig. S9.1. Schematic view of the theoretical model considered for the spreading-constriction impedance analysis.

To obtain the spreading-constriction impedance, first, the temperature profile inside the ceramic layer is obtained by solving the heat equation in the frequency domain in cylindrical coordinates,

$$
\frac{1}{r} \frac{\partial}{\partial r}\left(r \frac{\partial \theta}{\partial r}\right)+\frac{\partial^{2} \theta}{\partial x^{2}}=\frac{j \omega}{\alpha_{C}} \theta,
$$

where $r$ and $x$ are the radial and axial axes, respectively, $\alpha_{C}$ the thermal diffusivity of the ceramic material, $j=(-1)^{0.5}$ is the imaginary number, $\omega$ the angular frequency $(\omega=2 \pi f$, being $f$ the frequency), and $\theta$ is the temperature with respect to the ambient temperature $T_{i}$ in the frequency domain $\left[\theta=\mathscr{L}\left(T-T_{i}\right)\right]$.

To solve the heat equation, we need the boundary conditions of our system (see Fig. S9.1)

$$
\begin{aligned}
& \left(\frac{\partial \theta}{\partial r}\right)_{r=r C}=0 \quad \forall x, \\
& \left(\frac{\partial \theta}{\partial x}\right)_{x=0}=-\frac{\varphi_{0}}{\lambda_{C}} \quad \forall 0<r \leq r_{M},
\end{aligned}
$$




$$
\begin{aligned}
& \left(\frac{\partial \theta}{\partial x}\right)_{x=0}=0 \quad \forall r_{M}<r \leq r_{C}, \\
& \left(\frac{\partial \theta}{\partial x}\right)_{x=L_{C}}=-\frac{h_{3} \theta_{x=L_{C}}}{\lambda_{C}} \forall r,
\end{aligned}
$$

where $\lambda_{C}$ is the thermal conductivity of the ceramic layer, and $h_{3}$ is the heat transfer coefficient at $x=L_{C}$ (ideal heat sinks with a thermal contact resistivity $r_{T C}=1 / h_{3}$ can also be considered for contacted TE modules).

Following a similar procedure than Casalegno et al. [1], using the separation of variables, the temperature can be separated in two functions that only depend on one coordinate,

$$
\theta(x, r)=U(x) V(r) .
$$

Then, Eq. (A.9.1) can be written as,

$$
\frac{V^{\prime \prime}}{V}+\frac{1}{r} \frac{V^{\prime}}{V}-\frac{j \omega}{\alpha_{C}}=-\frac{U^{\prime \prime}}{U} .
$$

For all $x$ and $r$, both sides of Eq. (A.9.7) must be equal to a constant, which we choose to be $\gamma^{2}$. In addition, we only consider positive values of $\gamma^{2}$ to avoid oscillatory solutions of Eq. (A.9.11),

$$
-U^{\prime \prime}+\gamma^{2} U=0,
$$

$V^{\prime \prime}+\frac{1}{r} V^{\prime}+\left(\gamma^{2}-\frac{j \omega}{\alpha_{C}}\right) V=0$.

Defining another constant for Eq. (A.9.9),

$$
\beta^{2}=\gamma^{2}-\frac{j \omega}{\alpha_{C}},
$$

the solutions of the functions $U$ and $V$ can be written as,

$$
\begin{aligned}
& U(x)=C_{1} \cosh (\gamma x)+C_{2} \sinh (\gamma x), \\
& V(r)=C_{3} J_{0}(\beta r)+C_{4} Y_{0}(\beta r),
\end{aligned}
$$

where $J_{0}$ and $Y_{0}$ are zero order Bessel functions of first and second kind, respectively, and $C_{1}, C_{2}$, $C_{3}$, and $C_{4}$ are constants. It should be noticed that $C_{4}=0$ must be considered to obtain finite solutions, reducing Eq. (A.9.12) to, 
$V(r)=C_{3} J_{0}(\beta r)$,

Then, introducing Eq. (A.9.13) in Eq. (A.9.2), and using the property of Bessel functions,

$$
-J_{1}(r)=\frac{\partial J_{0}(r)}{\partial r},
$$

we obtain,

$$
\left[\frac{\partial V(r)}{\partial r}\right]_{r=r C}=C_{3}\left[\frac{\partial J_{0}(\beta r)}{\partial r}\right]_{r=r C}=C_{3} \beta\left[-J_{1}(\beta r)\right]_{r=r C}=0,
$$

where $J_{l}$ is the first order Bessel function of first kind.

Since $C_{3}$ cannot be zero, all the zeros of $J_{l}\left(\beta r_{C}\right)$ are solutions of Eq. (A.9.15). $J_{l}$ has infinite number of real zeros, each of them will be denoted as $\delta_{n}=\beta_{n} r_{C}$, such that,

$$
J_{1}\left(\delta_{n}\right)=J_{1}\left(\beta_{n} r_{C}\right)=0 .
$$

Thus, Eq. (A.9.10) becomes,

$$
\beta_{n}{ }^{2}=\gamma_{n}{ }^{2}-\frac{j \omega}{\alpha_{C}} \text {. }
$$

Hence, introducing Eq. (A.9.11) and Eq. (A.9.13) into Eq. (A.9.6), the temperature expression and its derivative take the form,

$$
\begin{aligned}
& \theta(x, r)=\sum_{n=1}^{\infty} J_{0}\left(\beta_{n} r\right)\left[C_{1, n} \cosh \left(\gamma_{n} x\right)+C_{2, n} \sinh \left(\gamma_{n} x\right)\right] \\
& \frac{\partial \theta(x, r)}{\partial x}=\sum_{n=1}^{\infty} J_{0}\left(\beta_{n} r\right) \gamma_{n}\left[C_{1, n} \sinh \left(\gamma_{n} x\right)+C_{2, n} \cosh \left(\gamma_{n} x\right)\right]
\end{aligned}
$$

Evaluating Eq. (A.9.18) and Eq. (A.9.19) at $\mathrm{x}=L_{C}$ and introducing the result in Eq. (A.9.5), after some algebraic steps, the ratio between $C_{2, n}$ and $C_{l, n}$ is obtained,

$$
R_{n}=-\frac{C_{2, n}}{C_{1, n}}=\left[\frac{\gamma_{n} \sinh \left(\gamma_{n} L_{C}\right)+\frac{h_{3}}{\lambda_{C}} \cosh \left(\gamma_{n} L_{C}\right)}{\gamma_{n} \cosh \left(\gamma_{n} L_{C}\right)+\frac{h_{3}}{\lambda_{C}} \sinh \left(\gamma_{n} L_{C}\right)}\right] \text {. }
$$

Eq. (A.9.18) can be rewritten as, 


$$
\theta(x, r)=\sum_{n=1}^{\infty} J_{0}\left(\beta_{n} r\right) C_{1, n}\left[\cosh \left(\gamma_{n} x\right)-R_{n} \sinh \left(\gamma_{n} x\right)\right],
$$

which can be derived and evaluated at $x=0$,

$$
\left(\frac{\partial \theta}{\partial x}\right)_{x=0}=\sum_{n=1}^{\infty}-J_{0}\left(\beta_{n} r\right) C_{1, n} R_{n} \gamma_{n}
$$

Introducing Eq. (A.9.22) in Eq. (A.9.3) and (A.9.4), multiplying by $r$ and $J_{o}\left(\beta_{m} r\right)$, and integrating from 0 up to $r_{C}$, we obtain,

$$
\int_{0}^{r_{C}} \sum_{n=1}^{\infty}\left(-C_{1, n} R_{n} \gamma_{n}\right) J_{0}\left(\beta_{n} r\right) J_{0}\left(\beta_{m} r\right) r \partial r=\int_{0}^{r_{M}}\left(-\frac{\varphi_{0}}{\lambda_{C}}\right) J_{0}\left(\beta_{m} r\right) r \partial r .
$$

Now, applying the orthogonality property,

$$
\int_{0}^{a} J_{0}\left(\delta_{n} \frac{r}{a}\right) J_{0}\left(\delta_{m} \frac{r}{a}\right) r \partial r=\frac{1}{2} a^{2}\left[J_{0}\left(\delta_{n}\right)\right]^{2} \delta_{n, m}
$$

to the left side of Eq. (A.9.23), and the integral identity,

$$
\int_{0}^{a} J_{0}\left(\beta_{m} r\right) r \partial r=\frac{a}{\beta_{m}} J_{1}\left(\beta_{m} a\right)
$$

to its right side, Eq. (A.9.23) becomes,

$$
\frac{1}{2} r_{C}^{2}\left[J_{0}\left(\beta_{n} r_{C}\right)\right]^{2}\left(-C_{1, n} R_{n} \gamma_{n}\right)=\left(-\frac{\varphi_{0}}{\lambda_{C}}\right) \frac{r_{M}}{\beta_{m}} J_{1}\left(\beta_{n} r_{M}\right) .
$$

Rearranging, we obtain,

$$
C_{1, n}=\frac{2 \varphi_{0} r_{M}}{\lambda_{C} \beta_{n} R_{n} \gamma_{n} r_{C}^{2}} \frac{J_{1}\left(\beta_{n} r_{M}\right)}{\left[J_{0}\left(\beta_{n} r_{C}\right)\right]^{2}}
$$

Eq. (A.9.20), (A.9.21), and (A.9.27) define the temperature distribution in the whole ceramic. Then, we average the temperature at $x=0$ in the ceramic for the area where the heat current is injected (the area of the metallic strip) with the expression,

$$
\bar{\theta}(0)=\frac{\int_{0}^{r_{M}} \theta(x, r) r \partial r}{\int_{0}^{r_{M}} r \partial r}=\frac{2}{r_{M}^{2}} \int_{0}^{r_{M}} \theta(x, r) r \partial r .
$$

Then, the average temperature is obtained by introducing Eq. (A.9.21) in Eq. (A.9.28). Then, using the integral identity given in Eq. (A.9.25), and evaluating at $x=0$, 
$\bar{\theta}(0)=\frac{2}{r_{M}^{2}} \sum_{n=1}^{\infty} C_{1, n} \frac{r_{M}}{\beta_{n}} J_{1}\left(\beta_{n} r_{M}\right)$

Finally, the spreading-constriction impedance is obtained as,

$z_{s / c}=\frac{\bar{\theta}(0)}{\varphi_{0}}=\frac{4}{\lambda_{C}} \sum_{n=1}^{\infty} \frac{J_{1}^{2}\left(\delta_{n} \frac{r_{M}}{r_{C}}\right)}{\gamma_{n} \delta_{n}{ }^{2} J_{0}{ }^{2}\left(\delta_{n}\right)}\left[\frac{\gamma_{n} \lambda_{C}+h_{3} \tanh \left(\gamma_{n} L_{C}\right)}{\gamma_{n} \lambda_{C} \tanh \left(\gamma_{n} L_{C}\right)+h_{3}}\right]$.

And the spreading-constriction resistance for steady state conditions $(\omega \rightarrow 0)$ becomes,

$r_{s / c}=\frac{4}{\lambda_{C}} \sum_{n=1}^{\infty} \frac{J_{1}{ }^{2}\left(\delta_{n} \frac{r_{M}}{r_{C}}\right) r_{C}}{\delta_{n}{ }^{3} J_{0}{ }^{2}\left(\delta_{n}\right)}\left[\frac{\delta_{n} \lambda_{C}+h_{3} r_{C} \tanh \left(\frac{\delta_{n} L_{C}}{r_{C}}\right)}{\delta_{n} \lambda_{C} \tanh \left(\frac{\delta_{n} L_{C}}{r_{C}}\right)+h_{3} r_{C}}\right]$.

\section{References}

[1] F. Casalegno, A. De Marchi, V. Giaretto, Frequency domain analysis of spreadingconstriction thermal impedance, Rev. Sci. Instrum. $84 \quad$ (2013) 024901. https://doi.org/10.1063/1.4789765. 


\section{List of figures}

Fig. 1.1. Percentage of primary energy (a) used, wasted in exhausts/effluents, and wasted in other ways, and (b) waste heat temperatures by sector. This figure was reprinted with permission [3].

Fig. 1.2. Schematic of the research strategy followed for the characterization of thermoelectric materials. 26

Fig. 1.3. Schematic of the research strategy followed for the characterization of thermoelectric modules.

Fig. 2.1. Schematic representation of the Seebeck effect, where two different metals (brown and grey wires) produce a potential difference when exposed to a temperature difference between its junctions.

Fig. 2.2. Schematic representation of the Peltier effect. The dashed line represents the electrochemical potential, the dotted line represents the energy of the conduction band edge in the thermoelectric material, and the solid line represents the average electron energy of the electrons flowing.

Fig. 2.3. Schematic representation of the influence of the charge carrier concentration in the Seebeck coefficient (red), electrical conductivity (green), $z T$ (blue), and thermal conductivity (orange). The horizontal dashed line represents the lattice thermal conductivity. 38

Fig. 2.4. Material's $z T$ as a function of temperature for the most common (a) p-type and (b) ntype thermoelectric materials, and (c, d) its peak evolution over the years, respectively. This figure was reprinted with permission [10].

Fig. 2.5. Price of the constituents for some of the most common thermoelectric materials (2016). This figure was reprinted with permission [15].

Fig. 2.6. (a) Schematic view of the typical structure of a thermoelectric device, and (b) an actual picture of a commercial Bi-Te device with a magnification of a pair. 42

Fig. 2.7. Theoretical heat-to-electricity energy conversion efficiency for thermoelectric modules with different $Z_{m} T$ values as a function of the hot side temperature, maintaining the cold side temperature at $300 \mathrm{~K}$. The simulations are made using Eq. (2.13).

Fig. 2.8. Theoretical coefficient of performance of thermoelectric modules with different $Z_{m} T$ values for (a) cooling and (b) heating applications. The simulations are made using Eq. (2.20) and Eq. (2.21), respectively.

Fig. 2.9. Signals applied and recorded during an impedance spectroscopy measurement, voltage (blue), and current (red) at a certain frequency. The equations represent the current $(I)$ and voltage $(V)$ sinusoidal signals for an angular frequency ( $\omega=2 \pi f$, being $f$ the frequency) as a function of time $t$. 
Fig. 2.10. Simulations from $1 \mathrm{mHz}$ to $1 \mathrm{MHz}$ of an impedance spectrum represented in a Nyquist plot (a) and Bode plots (b) and (c). The inset shows the equivalent circuit used to generate the data. For the simulations $R_{l}=0.5 \Omega, R_{2}=1 \Omega$, and $C_{l}=0.01 \mathrm{~F}$ were used. Frequency decreases from left to right in (a)

Fig. 2.11. Impedance spectroscopy simulations represented in Nyquist plots of the most common equivalent circuit elements for thermoelectric applications [resistor (a), capacitor (b), constant-temperature Warburg (c), and adiabatic Warburg (d)]. The insets show the symbols of the elements and the arrows indicate the characteristic parameters of each impedance element. The simulations were made using $R_{3}=1 \Omega, C_{l}=100 \mathrm{~F}, R_{4}=1.5 \Omega, \omega_{c r l}=1 \mathrm{rad} / \mathrm{s}, R_{5}=1 \Omega$, and $\omega_{c r 2}=0.01 \mathrm{rad} / \mathrm{s}$ and the equations presented in Table 2.1 . 51

Fig. 2.12. Schematic of a thermoelectric element contacted by two ceramic contacts. The arrows indicate the direction of the heat fluxes appearing at the junctions because of the Peltier effect when an n-type TE material and a positive current are considered. For the Peltier heat, the arrow point to the junction to indicate that electrons dissipate heat to the lattice. The blue solid line, the vertical dotted line, and the horizontal dashed line shows the temperature profile at steady state, the plane where the temperature remains constant, and the initial temperature profile, respectively. This figure was reprinted and modified with permission [42]. 52

Fig. 2.13. Simulated impedance spectra for a Bi-Te thermoelectric element with $S=190 \mu \mathrm{V} / \mathrm{K}$, $A=1.96 \mathrm{~mm}^{2}, L=1.64 \mathrm{~mm}, \alpha_{T E}=0.37 \mathrm{~mm}^{2} / \mathrm{s}$, and $\lambda_{T E}=1.5 \mathrm{~W} / \mathrm{Km}$ between two ceramic contacts of $L_{C}=0.57 \mathrm{~mm}, \alpha_{C}=10 \mathrm{~mm}^{2} / \mathrm{s}$, and $\lambda_{C}=35 \mathrm{~W} / \mathrm{Km}$ at $T i=294.7 \mathrm{~K}$. The total ohmic contribution $\left(R_{\Omega}=8.4 \mathrm{~m} \Omega\right)$, was approximated to the ohmic resistance of a single leg considering a resistivity $\rho_{T E}=0.01 \Omega \mathrm{mm}$. (a) Without the influence of the ceramic contacts and using the equivalent circuit in the inset. (b) Under contact influence and assuming that the Peltier flux is only conducted towards the ceramics $\left(\lambda_{T E}=0\right)$. The value of the ohmic resistance $R_{\Omega}$ has been subtracted in the real axis to gain clarity. (c) With contact influence using the equivalent circuit of the inset. The black line represents the same plot of (a) and the red line represents the same plot of (b) with the presence of $R_{\Omega}$. (d) The magnification of the high frequency region in plot (c) where $R_{\Omega}$ has also been subtracted in the real axis. .55

Fig. 2.14. Impedances of p-type thermoelements (a) with very thin Ag contacts and (b) with $\mathrm{Cu} /$ ceramic contacts. Line in (a) represents the fitting with the equivalent circuit in the inset of Fig. 2.13(a). The fitting provided $R_{\Omega}=84 \mathrm{~m} \Omega, R_{T E}=5.8 \mathrm{~m} \Omega$, and $\omega_{T E}=2.0 \mathrm{rad} / \mathrm{s}$. This figure was reprinted with permission [42]. 56

Fig. 2.15. Nyquist plot of a 254-leg thermoelectric module suspended in air. The inset shows a magnified high-frequency part. The line corresponds to the fitting with the equivalent circuit in the inset of Fig. 2.13(c). The fitting provided $R_{\Omega}=4.29 \Omega, R_{C}=0.149 \Omega, \omega_{C}=6.08 \mathrm{rad} / \mathrm{s}, R_{T E}=2.585$ $\Omega$, and $\omega_{T E}=0.24 \mathrm{rad} / \mathrm{s}$. This figure was reprinted with permission [42].

Fig. 3.1. Graphical abstract of the work: Thermal conductivity, electrical resistivity, and dimensionless figure of merit $(z T)$ determination of thermoelectric materials by impedance spectroscopy up to $250^{\circ} \mathrm{C}$. 66 
Fig. 3.2. Photograph of the sample holder employed for the impedance characterization of thermoelectric materials. The inset describes schematically how the sample is contacted.

Fig. 3.3. Variation of (a) the ohmic, the thermoelectric resistance, and (b) the extracted thermal conductivity, with different current amplitudes employed in the impedance experiments at $50{ }^{\circ} \mathrm{C}$ of Fig. S3.2. The inset in (a) shows the equivalent circuit used for the fittings.

Fig. 3.4. (a) Impedance spectroscopy measurements at different temperatures from one of the five measurement cycles performed. The lines represent the fittings to the experimental values. (b) Thermal conductivity, (c) electrical resistivity and (d) $z T$ values extracted from the impedance method and compared with results from different commercial equipment. The inset in (b) shows the Seebeck coefficient measured by the Linseis LSR-3 equipment, which is required to obtain the thermal conductivity by the impedance method. The error bars account for the total combined random errors, excluding the contribution from the specific heat for the laser flash case. 76

Fig. 4.1. Graphical abstract of the work: Complete Characterization of Thermoelectric Materials by Impedance Spectroscopy.

Fig. 4.2. Photograph of the sample holder employed. A schematic description of how the sample is contacted is provided in the inset.

Fig. 4.3. Impedance spectroscopy measurements at different temperatures from one of the five measurement cycles performed. The dots represent the experimental values and the lines represent the fit to these data. The inset shows the magnification of the high frequency part. 97

Fig. 4.4. (a) Electrical resistivity, (b) Seebeck coefficient, (c) thermal conductivity, and (d) $z T$ values extracted from the impedance method and compared with results from different commercial equipment. The error bars account for the total combined random errors $\left(u_{c}\right)$, excluding the contribution from the specific heat for the laser flash case. The confidence interval is $1 \sigma$. .98

Fig. 5.1. Graphical abstract of the work: Influence of convection at outer ceramic surfaces on the characterization of thermoelectric modules by impedance spectroscopy. 110

Fig. 5.2. Thermal model employed in the theoretical analysis. A positive value of both the current and the Seebeck coefficient is considered. The arrows indicate the direction of the heat fluxes appearing at the boundaries. For Peltier heat, the arrows point out of the junction when the electrons absorb heat from the lattice. The solid line qualitatively depicts a possible thermal profile. The dotted line shows the plane where the temperature remains constant at any time and the dashed line indicates the initial temperature.

Fig. 5.3. (a) Equivalent circuit obtained when convection effects are considered at the outer surface of the ceramics in a thermoelectric module. (b) Simplified equivalent circuit for standard commercial thermoelectric modules. The equivalent circuit elements framed in the dotted line are related to the ceramic plates. The ones framed by the solid line in grey correspond to the thermoelement. 
Fig. 5.4. (a) Impedance simulations in the $10 \mathrm{mHz}$ to $10 \mathrm{kHz}$ frequency range of the equivalent circuit elements $Z_{W a}$ (red), $Z_{W C T}$ (black) and the parallel combination of $Z_{W a}$ and $R_{\text {conv }}$ (green). The plots in the inset show magnifications at medium and high frequencies. Simulations in (b) represent the complete equivalent circuit for a thermoelectric module [Fig. 5.3(b)] under high vacuum $\left(h=0 \mathrm{WK}^{-1} \mathrm{~m}^{-2}\right)$ and room $\left(h \approx 25 \mathrm{WK}^{-1} \mathrm{~m}^{-2}\right)$ conditions. $R_{\Omega}=0$ is considered for simplicity and typical values for commercial thermoelectric modules were used $\left(S=190 \mu \mathrm{VK}^{-1}, \lambda_{T E}=1.5 \mathrm{WK}^{-}\right.$ ${ }^{1} \mathrm{~m}^{-1}, \alpha_{T E}=0.37 \mathrm{~mm}^{2} \mathrm{~s}^{-1}, L=1.6 \mathrm{~mm}, \lambda_{C}=35 \mathrm{WK}^{-1} \mathrm{~m}^{-1}, \alpha_{C}=10 \mathrm{~mm}^{2} \mathrm{~s}^{-1}, L_{C}=0.6 \mathrm{~mm}, N=127, A=1.94$ $\left.\mathrm{mm}^{2}, T_{i}=294.7 \mathrm{~K}, \eta=0.3\right)$. 117

Fig. 5.5. Experimental impedance spectra of a suspended commercial thermoelectric module at room (ambient pressure) and high vacuum conditions (dots) and their corresponding fittings to the equivalent circuit of Fig. 5.3(b) (lines). The convection resistance was not considered for the vacuum case $\left(h \approx 0 \mathrm{WK}^{-1} \mathrm{~m}^{-2}\right)$. The quantity of $13.8 \mathrm{~m} \Omega$ was subtracted to the real impedance part of the vacuum measurement in order to match the ohmic resistances $\left(R_{\Omega}\right)$ and obtain a clearer comparison. 118

Fig. 6.1. Graphical abstract of the work: Characterization of thermal contacts between heat exchangers and a thermoelectric module by impedance spectroscopy. 126

Fig. 6.2. Thermal model employed in the theoretical analysis. The arrows indicate the direction of the conducting heat fluxes appearing at the different junctions, considering a positive value of both the electrical current and the Seebeck coefficient. For the Peltier heat, the arrows point out of the junction when heat is absorbed. The solid line depicts qualitatively a possible thermal profile. The dotted line shows the plane where the temperature remains constant at any time due to the symmetry of the system. The dashed line indicates the initial temperature. 129

Fig. 6.3. Equivalent circuit corresponding to a thermoelectric device contacted by two heat sinks, existing a thermal contact resistance at the contact. The equivalent circuit elements framed by the dotted line are related to the external ceramic plates. The elements framed by the solid line relate to the thermoelements. 132

Fig. 6.4. (a) Impedance spectroscopy simulations from $10 \mathrm{mHz}$ to $10 \mathrm{kHz}$ for four different thermal contact resistivity values for the contacts between a thermoelectric module and two heat sinks. The plots in (b) and (c) are magnifications of the bottom left part (same axis units). Typical values for commercial $\mathrm{Bi}_{2} \mathrm{Te}_{3}$ thermoelectric modules were used $\left(N=127, S=180 \mu \mathrm{VK}^{-1}, \rho_{T E}=1\right.$ $\mathrm{m} \Omega \mathrm{cm}, \lambda_{T E}=1.5 \mathrm{Wm}^{-1} \mathrm{~K}^{-1}, \alpha_{T E}=0.37 \mathrm{~mm}^{2} \mathrm{~s}^{-1}, L=1.2 \mathrm{~mm}, \lambda_{C}=20 \mathrm{Wm}^{-1} \mathrm{~K}^{-1}, \alpha_{C}=10 \mathrm{~mm}^{2} \mathrm{~s}^{-1}, L_{C}=0.7$ $\left.\mathrm{mm}, A=1.69 \mathrm{~mm}^{2}, T=300 \mathrm{~K}, \eta=0.268\right)$. Frequency decreases from left to right.

Fig. 6.5. Schematic of the experimental setups for the measurements performed to (a) a module suspended, and (b) the same module in contact with two aluminum blocks acting as heat exchangers and contacted with and without heat sink compound, and with and without additional weight on top.. 135

Fig. 6.6. Experimental impedance spectra (dots) of a commercial $\mathrm{Bi}_{2} \mathrm{Te}_{3}$ thermoelectric module suspended in vacuum and in contact in air with two aluminum blocks (with and without heat sink compound, and with additional masses on top of the top aluminum block). The lines 
represent the fittings performed with the equivalent circuit of Fig. 6.3. The inset shows the magnification at medium and high frequencies. Frequency decreases from left to right. 136

Fig. 7.1. Graphical abstract of the work: Detailed assessment of thermoelectric modules by a comprehensive impedance spectroscopy equivalent circuit. 146

Fig. 7.2. Schematic view of the theoretical model considered in this analysis. A positive value of Seebeck coefficient and current is considered to represent the temperature profile (blue solid lines). The dotted line shows the plane where the temperature remains constant and the dashed line represents the initial temperature profile.

Fig. 7.3. Comprehensive equivalent circuit with all the key phenomena that can occur in thermoelectric devices. 156

Fig. 7.4. Impedance spectroscopy simulations when $L_{M}=0$ for different values of the filling factor $\eta$, and thermal contact resistivity $r_{T C I}$. The four larger spectra simulations consider a module suspended in vacuum, and the other four consider it in contact with ideal heat sinks with a thermal contact resistivity $r_{T C}=1 / h_{3}=10^{-4} \mathrm{~m}^{2} \mathrm{KW}^{-1}$. Plots (b) and (c) are magnifications of the bottom left part in (a) (same axis units). Plots (d) and (e) are magnifications for the suspended and contacted configurations, respectively. 159

Fig. 7.5. Simplified equivalent circuits of thermoelectric modules when $h_{2}=0, L_{M}=0$, and $r_{T C 2}=0$ for (a) contacted modules and (b) suspended modules in vacuum $\left(h_{3}=0\right)$. 160

Fig. 7.6. (a, b, c) Impedance spectroscopy simulations when $L_{M}=0$ for different values of $r_{T C l}$ and $\eta=0.3$. (d, e, f) Impedance spectroscopy simulations when $L_{M}=0$ for different values of $\eta$ and $r_{T C I}=10^{-5} \mathrm{~m}^{2} \mathrm{KW}^{-1}$. The plots in (b), (c), (e), and (f) are magnifications of the bottom left part (same axis units). The four larger spectra simulations in each plot consider a module suspended in vacuum, and the other four consider it in contact with ideal heat sinks with a thermal contact resistivity $r_{T C}=1 / h_{3}=10^{-4} \mathrm{~m}^{2} \mathrm{KW}^{-1}$ 162

Fig. 7.7. Impedance spectroscopy simulations for different values of $L_{M}$ for (a-e) $r_{T C l}=0$ $\mathrm{m}^{2} \mathrm{KW}^{-1}$ and (f-j) for $r_{T C l}=10^{-5} \mathrm{~m}^{2} \mathrm{KW}^{-1}$. (k-o) Simulations for different values of $r_{T C l}$ considering a fixed value of $L_{M}=0.5 \mathrm{~mm}$. In all cases $\eta=0.3$, and $r_{T C 2}=0 \mathrm{~m}^{2} \mathrm{KW}^{-1}$. The plots (b-e), (g-j) and (lo) are magnifications of the bottom left part (same axis units) of plots (a), (f), and (k), respectively. The four larger spectra simulations of each graph consider the module suspended in vacuum, and the other four consider it in contact with a heat source/sink with a thermal contact resistivity of $10^{-4} \mathrm{~m}^{2} \mathrm{KW}^{-1}\left(r_{T C}=1 / h_{3}=10^{-4} \mathrm{~m}^{2} \mathrm{KW}^{-1}\right)$. The four larger spectra simulations in each plot consider a module suspended in vacuum, and the other four consider it in contact with ideal heat sinks with a thermal contact resistivity $r_{T C}=1 / h_{3}=10^{-4} \mathrm{~m}^{2} \mathrm{KW}^{-1}$ 165

Fig. 7.8. Simplified equivalent circuits of thermoelectric modules when $h_{2}=0, r_{T C l}=0$, and $r_{T C 2}=0$ for $(\mathrm{a})$ contacted modules and (b) suspended modules in vacuum $\left(h_{3}=0\right)$. 166

Fig. 7.9. Simplified equivalent circuits of thermoelectric modules when $h_{2}=0$, and $r_{T C 2}=0$ for (a) contacted modules and (b) suspended modules in vacuum $\left(h_{3}=0\right)$. 166 
Fig. 7.10. (a-c) Impedance spectroscopy simulations for different values of $r_{T C 2}, \eta=0.3$, and $L_{M}=0.5 \mathrm{~mm}$. (d-f) Impedance simulations for different values of $\eta, L_{M}=0.5 \mathrm{~mm}$, and $r_{T C 2}=10^{-5}$ $\mathrm{m}^{2} \mathrm{KW}^{-1}$. (g-i) Impedance simulations for different values of $L_{M}, \eta=0.3$, and $r_{T C 2}=10^{-5} \mathrm{~m}^{2} \mathrm{KW}^{-1}$. For all cases $r_{T C l}=0 \mathrm{~m}^{2} \mathrm{KW}^{-1}$. Plots $(\mathrm{b}, \mathrm{c}),(\mathrm{e}, \mathrm{f})$, and $(\mathrm{h}, \mathrm{i})$ are magnifications of the bottom left part (same axis units) of plots (a), (d), and (g), respectively. The four larger spectra simulations in each plot consider a module suspended in vacuum, and the other four consider it in contact with ideal heat sinks with a thermal contact resistivity $r_{T C}=1 / h_{3}=10^{-4} \mathrm{~m}^{2} \mathrm{KW}^{-1}$. 168

Fig. 7.11. Simplified equivalent circuits of thermoelectric modules when $h_{2}=0$, and $r_{T C l}=0$ for (a) contacted modules and (b) suspended modules in vacuum $\left(h_{3}=0\right)$.

Fig. 7.12. Simplified equivalent circuits of thermoelectric modules when $h_{2}=0$ for (a) contacted modules and (b) suspended modules in vacuum $\left(h_{3}=0\right)$. 170

Fig. 7.13. (a-c) Impedance spectroscopy simulations for different values of $r_{T C l}, L_{M}=0.5 \mathrm{~mm}$, and $r_{T C 2}=10^{-5} \mathrm{~m}^{2} \mathrm{KW}^{-1}$. (d-f) Impedance simulations for different values of $L_{M}, r_{T C l}=10^{-5} \mathrm{~m}^{2} \mathrm{KW}^{-}$ , and $r_{T C 2}=10^{-5} \mathrm{~m}^{2} \mathrm{KW}^{-1}$. (g-i) Impedance simulations for different values of $r_{T C 2}, L_{M}=0.5 \mathrm{~mm}$, and $r_{T C l}=10^{-5} \mathrm{~m}^{2} \mathrm{KW}^{-1}$. For all cases $\eta=0.3$. Plots $(\mathrm{b}, \mathrm{c}),(\mathrm{e}, \mathrm{f})$, and $(\mathrm{h}, \mathrm{i})$ are magnifications of the bottom left part (same axis units) of plots (a), (d), and (g), respectively. The four larger spectra simulations in each plot consider a module suspended in vacuum, and the other four consider it in contact with ideal heat sinks with a thermal contact resistivity $r_{T C}=1 / h_{3}=10^{-4} \mathrm{~m}^{2} \mathrm{KW}^{-1}$

Fig. 7.14. (a) Experimental impedance spectroscopy measurement of Module 1, including the magnification of the high frequency part in the inset. (b) Impedance simulations performed using the Matlab code in Annex 9.2 for different values of thermal contact resistivities $r_{T C l}$ and $r_{T C 2}$. (ce) Magnifications of the high frequency part of different spectra in (b). Dashed lines are indications of a $45^{\circ}$ slope. The simulations were made with the geometric parameters of columns 3 to 9 of Table 7.1 and the default material's properties previously used in the equivalent circuit analysis of section 7.3. The ohmic resistance has been omitted in the simulations for clarity. 174

Fig. 7.15. (a) Experimental impedance spectroscopy measurement of Module 2, including the magnification of the high frequency part in the inset. (b) Impedance simulations performed using the Matlab code in Annex 9.2 for different values of thermal contact resistivities $r_{T C l}$ and $r_{T C 2}$. (ce) Magnifications of the high frequency part of different spectra in (b). Dashed lines are indications of a $45^{\circ}$ slope. The simulations were made with the geometric parameters of columns 3 to 9 of Table 7.1 and the default material's properties previously used in the equivalent circuit analysis of section 7.3. The ohmic resistance has been omitted in the simulations for clarity. 175

Fig. 7.16. (a) Experimental impedance spectroscopy measurement of Module 1, including the magnification of the high frequency part in the inset. (b) Impedance simulations performed using the Matlab code in Annex 9.2 for different values of thermal contact resistivities $r_{T C l}$ and $r_{T C 2}$. (ce) Magnifications of the high frequency part of different spectra in (b). Dashed lines are indications of a $45^{\circ}$ slope. The simulations were made with the geometric parameters of columns 3 to 9 of Table 7.1 and the default material's properties previously used in the equivalent circuit analysis of section 7.3. The ohmic resistance has been omitted in the simulations for clarity. 176 


\section{List of tables}

Table 2.1. Name, impedance function, and reference to the representation in the complex plane of the most common equivalent circuit elements for thermoelectric applications. 50

Table 3.1. Average values with their associated random, systematic and total errors of the thermoelectric properties of a skutterudite sample obtained by the impedance spectroscopy method. 78

Table 4.1. Average values with their associated random, systematic and total errors of the thermoelectric properties of the skutterudite sample obtained by the impedance spectroscopy method. 101

Table 5.1. Fitting parameters obtained from the fittings to the experimental measurements in Fig. 5.5 of a commercial thermoelectric module under high vacuum and ambient pressure conditions (no vacuum). The errors provided from the fitting are given in brackets. The Seebeck coefficient was experimentally obtained and the convection heat transfer coefficient calculated from the no vacuum fitting result

Table 6.1. Fitting parameters obtained from the fittings to the experimental measurements of Fig. 6.6 of a commercial $\mathrm{Bi}_{2} \mathrm{Te}_{3}$ thermoelectric module suspended under vacuum and in contact with two aluminum blocks at room conditions $(\mathrm{T}=298.0 \mathrm{~K})$, with and without using heat sink compound, and with additional masses on top. The errors provided from the fittings are given between brackets. The thermal contact resistivities were calculated using Eq. (5.13) and the Seebeck coefficient value of $191.53 \mu \mathrm{VK}^{-1}$ was experimentally obtained

Table 7.1. Specifications of the commercial TE modules used in this study and experimental impedance spectroscopy parameters employed. 